\title{
A Low-Power Optoelectronic Characterizer for CubeSat: LOCC and III-V Nitride Based LEDs
}

\author{
Matthew J. Pachol
}

Follow this and additional works at: https://researchrepository.wvu.edu/etd

\section{Recommended Citation}

Pachol, Matthew J., "A Low-Power Optoelectronic Characterizer for CubeSat: LOCC and III-V Nitride Based LEDs" (2017). Graduate Theses, Dissertations, and Problem Reports. 6366.

https://researchrepository.wvu.edu/etd/6366

This Thesis is protected by copyright and/or related rights. It has been brought to you by the The Research Repository @ WVU with permission from the rights-holder(s). You are free to use this Thesis in any way that is permitted by the copyright and related rights legislation that applies to your use. For other uses you must obtain permission from the rights-holder(s) directly, unless additional rights are indicated by a Creative Commons license in the record and/ or on the work itself. This Thesis has been accepted for inclusion in WVU Graduate Theses, Dissertations, and Problem Reports collection by an authorized administrator of The Research Repository @ WVU. For more information, please contact researchrepository@mail.wvu.edu. 


\title{
A Low-Power Optoelectronic Characterizer for CubeSat: LOCC and III-V Nitride Based LEDs
}

\author{
Matthew J. Pachol \\ B.S. Electrical Engineering, West Virginia University, Morgantown, WV
}

Thesis Submitted to the

Benjamin M. Statler College of Engineering and Mineral Resources

at West Virginia University

in partial fulfillment of the requirements for the degree of

\author{
Master of Science \\ in \\ Electrical Engineering
}

Jeremy M. Dawson, Ph.D., Chair

David W. Graham, Ph.D.

Dimitris Korakakis, Ph.D.

Lane Department of Computer Science and Electrical Engineering

Morgantown, WV

2017

Keywords: Light Emitting Diode, CubeSat, Optoelectronic Characterization, Ionizing Radiation, Low-power

Copyright (C) 2017 Matthew J. Pachol 


\title{
Abstract \\ A Low-Powered Optoelectronic Characterizer for CubeSat: LOCC and III-V Nitride Based LEDs
}

\author{
Matthew J. Pachol
}

III-V semiconductor materials exhibit robustness and natural hardness when exposed to ionizing radiation and temperature swings. With these characteristics in mind, III-V Nitride Light Emitting Diodes (LEDs) are ideal devices for space-based applications and missions. The effects of ionizing radiation on optoelectronic devices comprised of III-V materials have been studied, but results have been obtained through experiments performed in terrestrial laboratories. While these laboratory tests may lend insight into device lifetimes, performance degradation, etc., they are no substitute for similar measurements and characterization performed in space.

Interest in small satellite applications have grown over the past decade. These solutions range from Earth imaging to communication networks. Small satellites provide a unique opportunity to gain an understanding of the reliability and operational characteristics of III-V based materials and other semiconductor devices while exposed to the environment of space. To meet the constraints of the small satellite, a Low-powered Optoelectronic Characterizer for CubeSat (LOCC) has been developed in PC/104 form, measuring 3.6 by 3.8 inches. LOCC performs current-voltage and electroluminescent measurements of LEDs while in space. The LOCC system is designed using low-power integrated circuits that can supply over $100 \mathrm{~mA}$ of current to LEDs while maintaining low power of 3.2W under operation.

This thesis presents the design, implementation, and control of the LOCC system. This includes system block diagrams, printed circuit board layouts, interfacing, firmware, and software. Additionally, the resulting current-voltage measurements, required wattage, and required data storage are presented to illustrate functionality. This instrumentation enables the study of optoelectronic devices in space, allowing future research to focus on producing radiation hard light emitting devices that can operate in environments with reduced shielding against ionizing radiation while maintaining device reliability. 


\section{Acknowledgements}

I would like to thank my academic and research advisor, Dr. Jeremy Dawson, for giving me the opportunity to be a graduate research assistant. Dr. Dawson has given his patience, encouragement, and his trust, allowing me to utilize my creativity as an engineer and see this project through. Without him, I would not have had this grand opportunity to put this work into space and be a part of West Virginia's first spacecraft. I also give my appreciation to Dr. David Graham, who has given me the knowledge and confidence to approach analog and digital circuitry. I would also like to thank Dr. Dimitris Korakakis, who has given me support and advice in the field of electrical engineering and helped provide guidance on the development of the LOCC system.

I'd like to dedicate this work to my family starting with my father, Stephen Pachol, my mother, Sally Pachol, and my brother, Marcus Pachol, for their love, support, and patience. I dedicate this work to my other half and best friend, Jourdan Gibson, for being there for me through this process.

I thank WVU faculty members for helping me through graduate school. I thank my colleagues and lab mates from the nanophotonic and biometrics labs, NASA IV\&V, WVU Shared facilities, and friends for their support. A special thank you to Steve White and Matt Grubb for making the LOCC system a reality. Also thank you to Jason Battin, Scott Zemerick, Harley Hart, Anand Kadiyala, Stallone Sabatier, Kyle Smith, Casey Norville, Kenneth Hite, Morgan Trester, Yasser Khouj, James Searls, Christo Robison, Douglas Kerr, Seth Leffel, and Catherine O'Hearn. 


\section{Table of Contents}

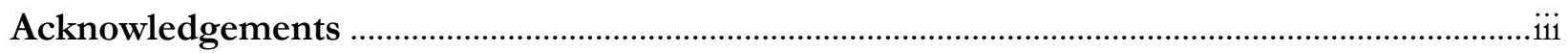

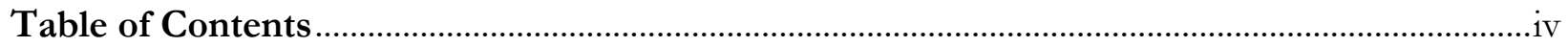

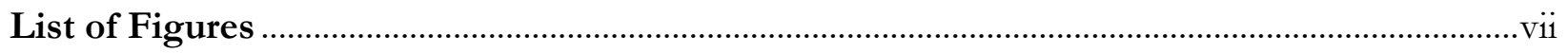

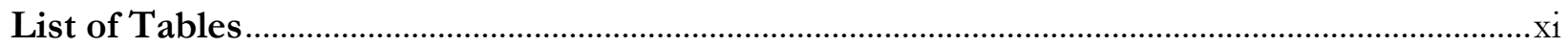

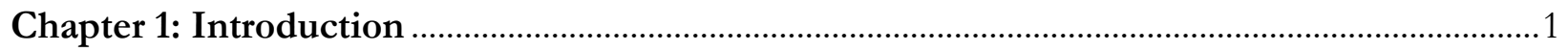

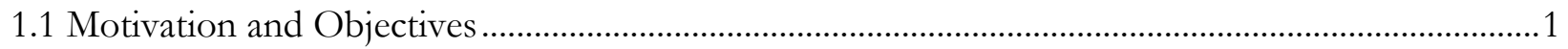

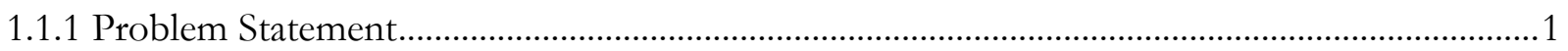

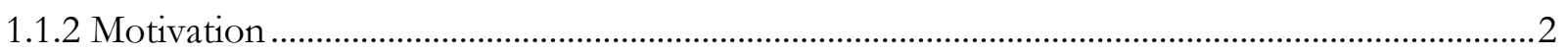

1.1.2.1 Optoelectronics in Small Satellite Applications................................................................

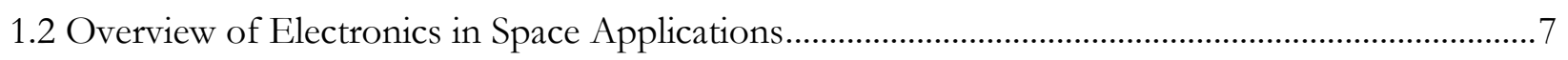

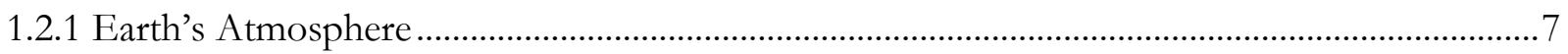

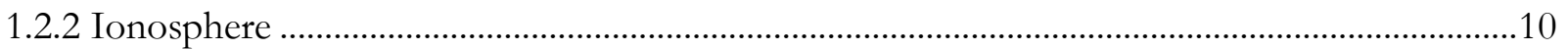

1.2.3 Radiation Effects on Solid-State Electronics ..........................................................................11

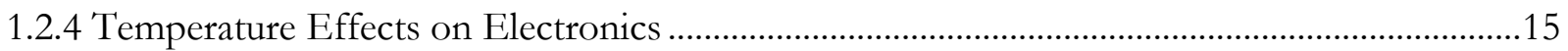

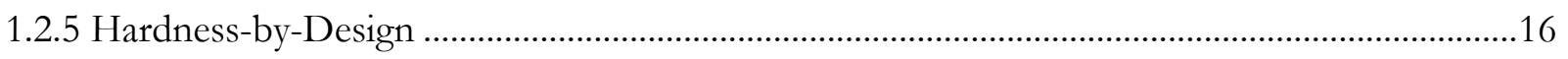

1.3 Overview of GaN Materials for Space Applications......................................................................17

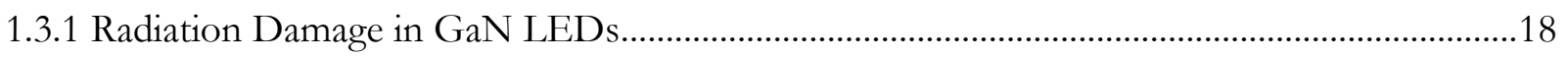

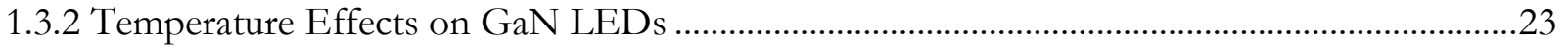

1.3.3 Annealing Effecting on Radiation Damage...............................................................................26

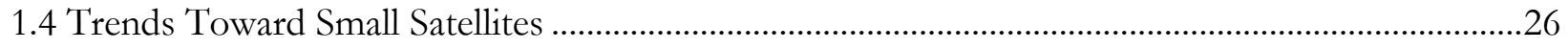

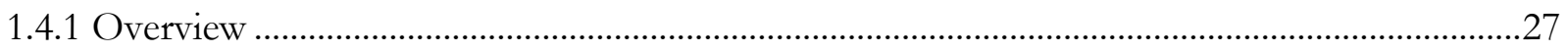

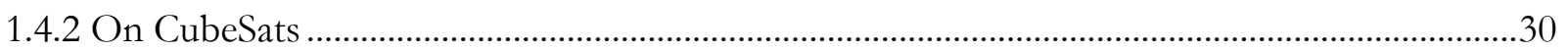

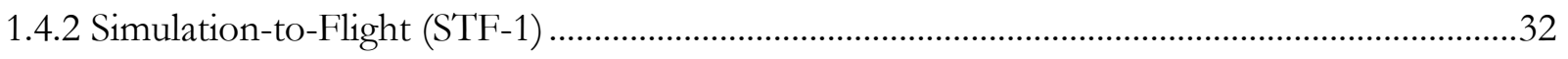

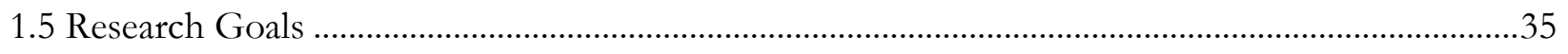

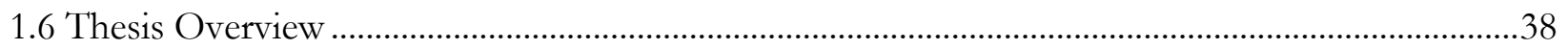




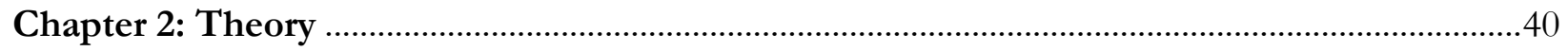

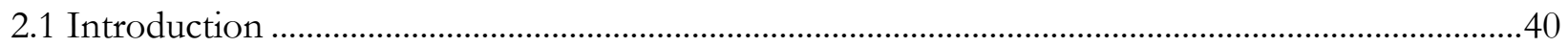

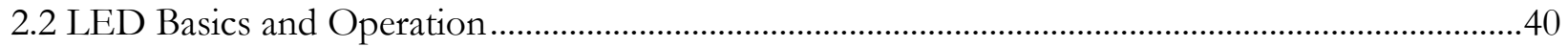

2.2.1 Direct and Indirect Band Gap Semiconductors .............................................................................. 41

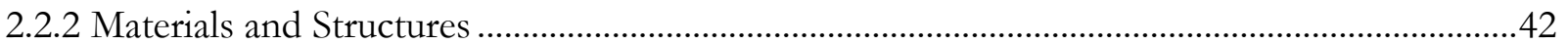

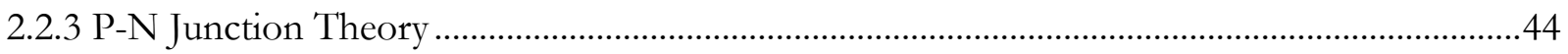

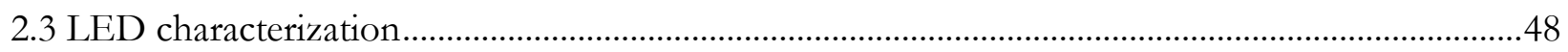

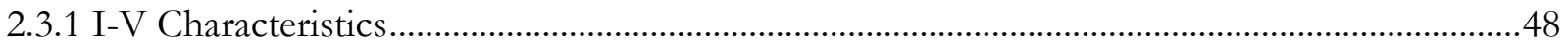

2.3.2 Electroluminescent (EL) Characterization ..................................................................................50

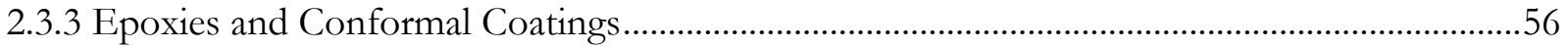

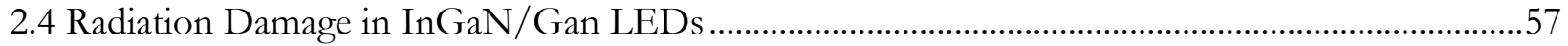

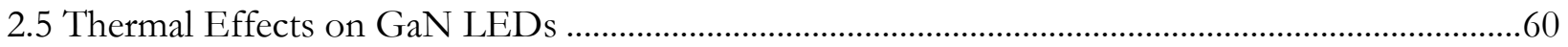

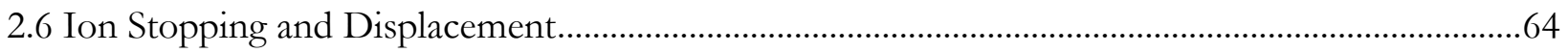

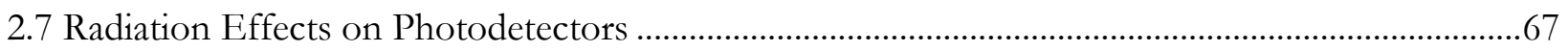

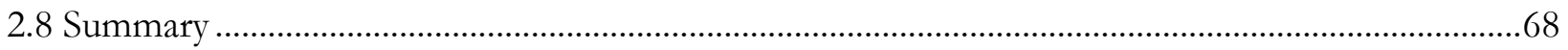

Chapter 3: System Design and Experimental Test Plan ............................................................ 70

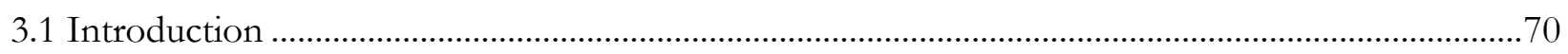

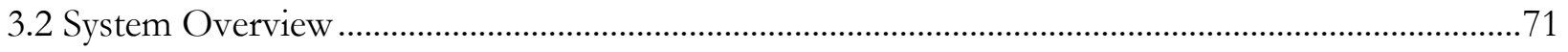

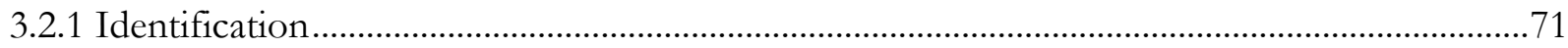

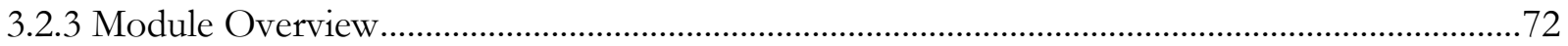

3.2.3.1 LED Characterization/MCU module ...................................................................................... 73

3.2.3.2 Electroluminescence Characterization Module ....................................................................74

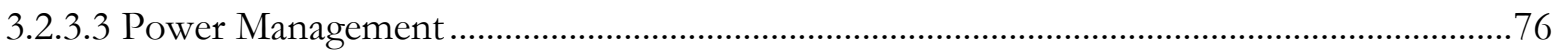

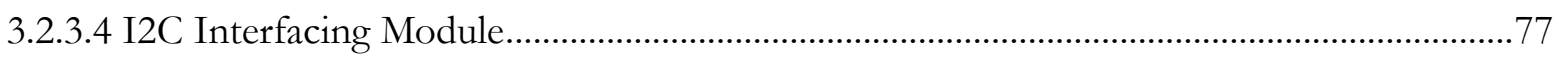

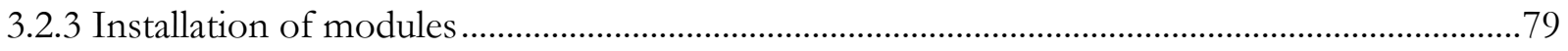

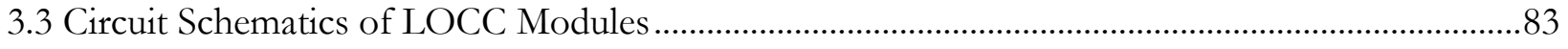

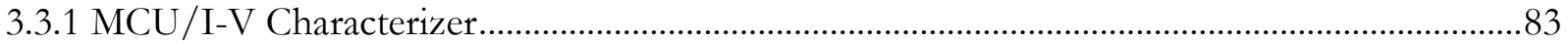

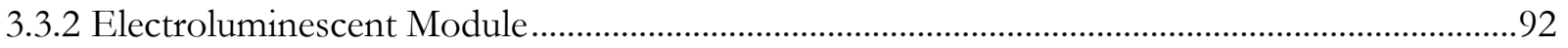

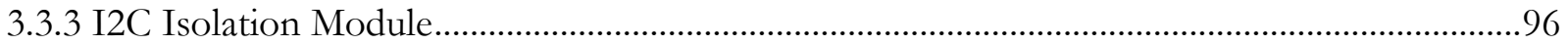

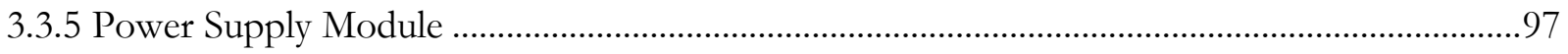

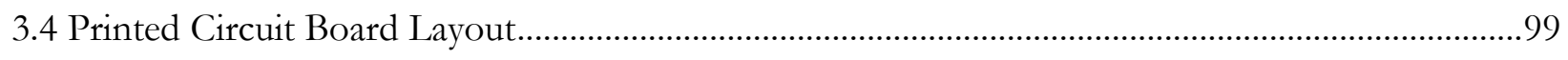

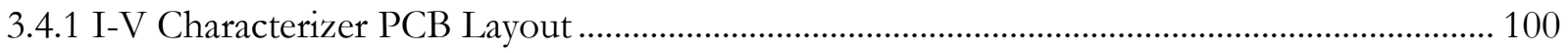




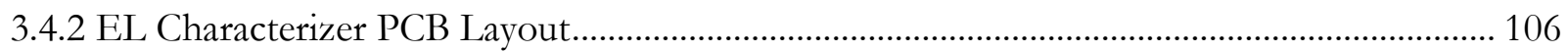

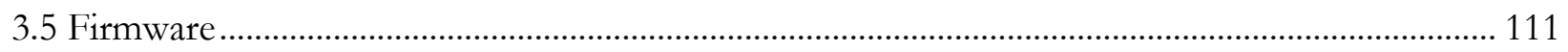

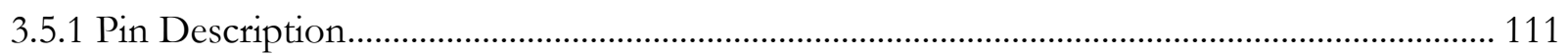

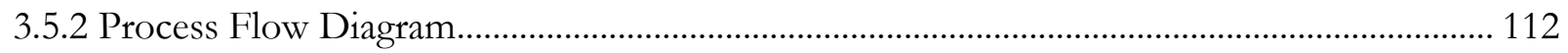

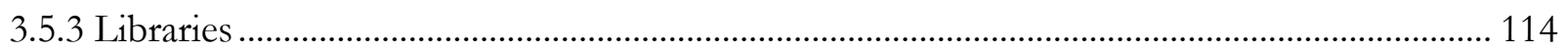

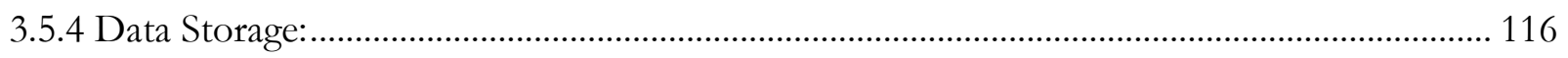

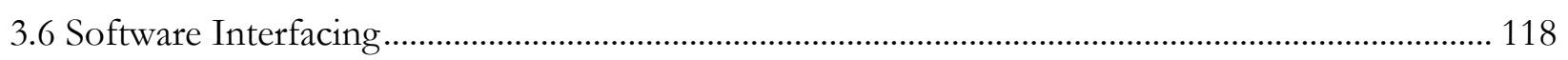

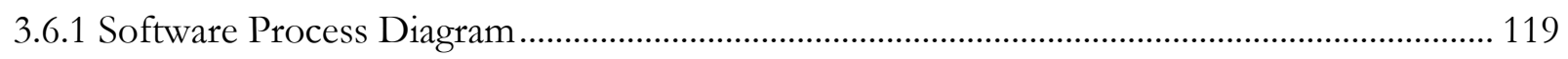

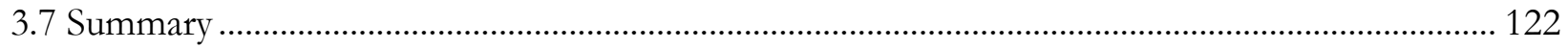

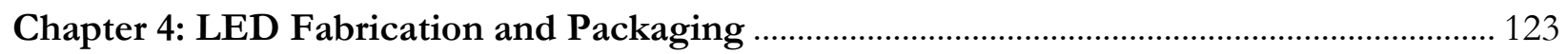

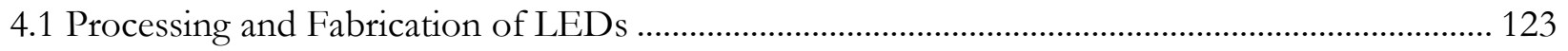

4.2 Integration and Packaging of LEDs ............................................................................................... 132

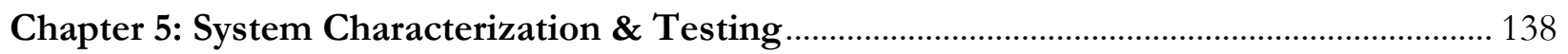

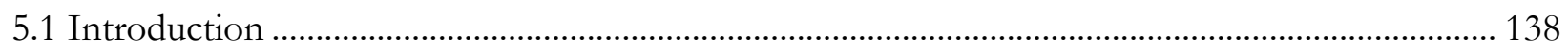

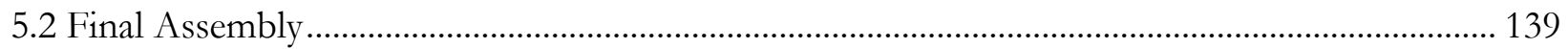

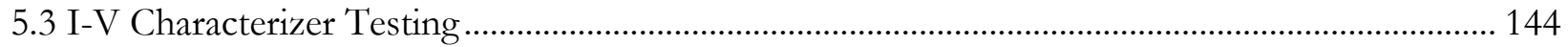

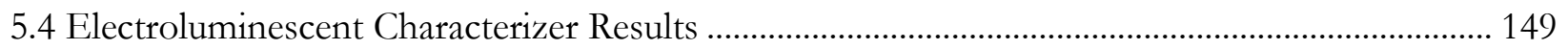

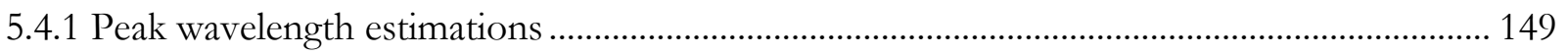

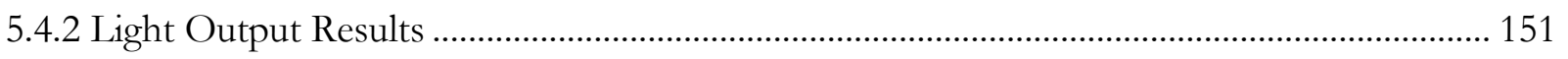

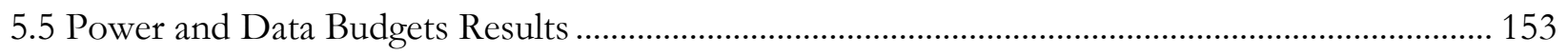

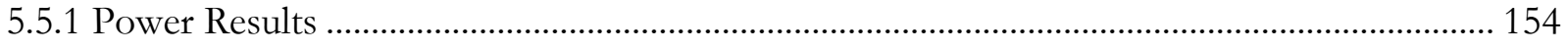

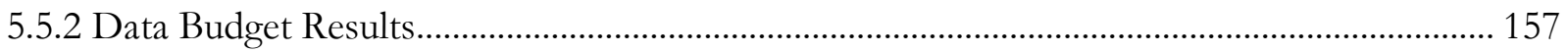

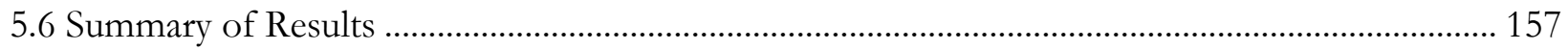

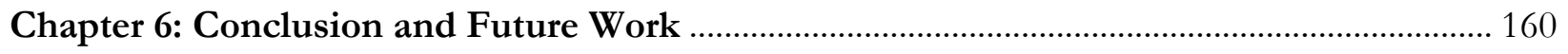

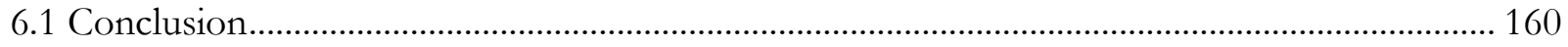

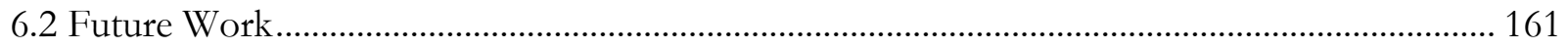

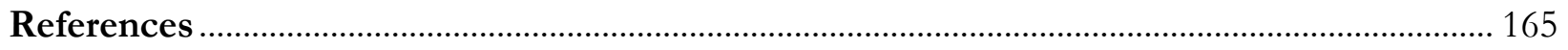

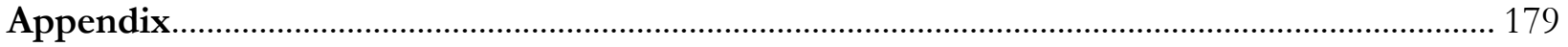




\section{List of Figures}

Figure 1. 1: Illustration of MRO instrumentation [71] .........................................................................4

Figure 1. 2: (LEFT)Mars Color Imager, (RIGHT) HiRISE Telescope Camera [82].............................. 4

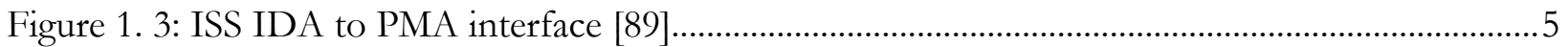

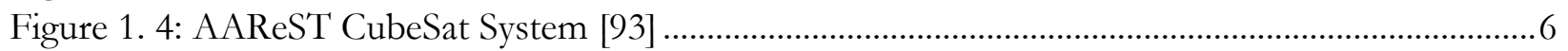

Figure 1. 5: Illustration of Earth's atmospheric boundaries [94] ........................................................... 8

Figure 1. 6: Basic Quantities of Ionosphere as function of height [101] ..............................................11

Figure 1. 7: Atomic Displacement and point defects (vacancy and interstitial).....................................12

Figure 1. 8: Space Charge and Forward voltage as function of proton fluence [22] ............................13

Figure 1. 9: Reverse Current as versus bias voltage with varying thicknesses and irradiation [21]......14

Figure 1. 10: linear fit of multiple silicon materials after electron irradiation after 8 minutes of annealing

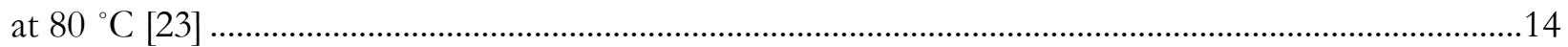

Figure 1. 11: DC Current Gain of npn and pnp transistors [24] …………........................................15

Figure 1. 12: (LEFT) I-V Characteristics of SiGe diode as a function of temperature, (RIGHT) I-V

characteristics of Si MOSFETs as functions of temperature [25] .........................................................16

Figure 1. 13: Normalized output vs Proton Irradiation Energies [41] ..................................................19

Figure 1. 14: Light Output after $10 \mathrm{MeV}$ proton irradtion of $\mathrm{GaN}$ and GaAs materials [41] .............19

Figure 1. 15: Current-Voltage Characteristics after irradiation [42] .......................................................20

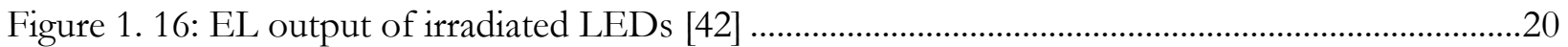

Figure 1. 17: Normalized light output vs. electron irradiation [43] ........................................................22

Figure 1. 18: (LEFT) Resistive change versus temperature, (RIGHT) Forward Voltage Change versus

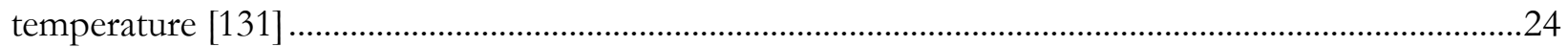

Figure 1. 19: Junction temperature as a function of current at different temperatures [131]..............24

Figure 1. 20: Emission shift in peak wavelength as a function of junction temperature [133] ...........25

Figure 1. 21: Nano/Microsatellite Projection and History. SpaceWorks 2016 [114] ...........................29

Figure 1. 22: Various CubeSat Configurations [120] ............................................................................

Figure 1. 23: P-POD Deployment System and Cross-Sectional Area [7]..............................................32

Figure 1. 24: NanoRack's CubeSat Deployer (NRCSD) [121] [122] .......................................................32 Figure 1. 25: (LEFT) 3D Rendering of Skeleton STF-1, (RIGHT) 3D Rendering of STF-1 Assembled.

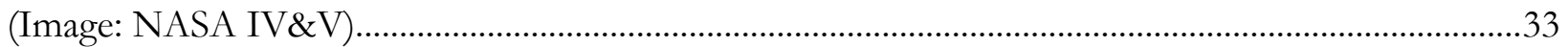

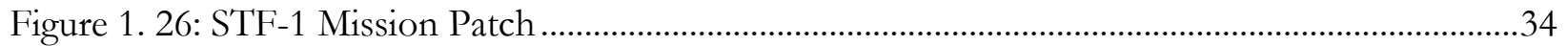

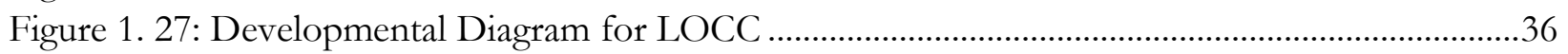

Figure 1. 28: Operational Diagram of LOCC system................................................................................

Figure 2. 1: Electromagnetic Spectrum [127] ....................................................................................

Figure 2. 2: Energy Band Diagram of Direct (Gallium Nitride) and Indirect Bandgap (Silicon Carbide)

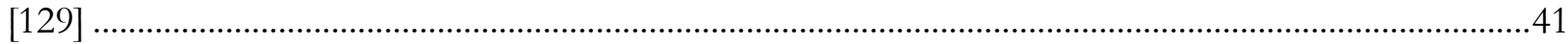

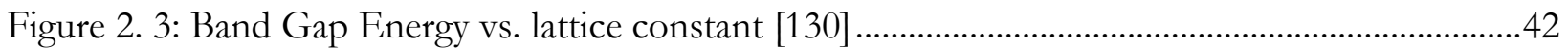


Figure 2. 4: (LEFT)Wutzite Crystal Stucture, (RIGHT) Zinc Blende Crystal Structure [133].

Figure 2. 5: Asymmetric p-doped P-N junction at thermal equilibrium and zero-bias voltage [134] .45

Figure 2. 6: LED Circuit and band Diagram [135] ..........................................................................46

Figure 2. 7: Emissions and Absorption operations of p-n junction........................................................47

Figure 2. 8: Schottky Diode I-V characteristic curve [145] ........................................................................49

Figure 2. 9: Illustration of WVU LED I-V Characterization Curves ..................................................50

Figure 2. 10: Electroluminescent response of blue/green light source [146] ….....................................51

Figure 2. 11: EL spectral output of WVU LEDs .....................................................................................52

Figure 2. 12: Responsivity of XYZ color sensor MTCSiCF [163] ........................................................53

Figure 2. 13: CIE 1931 Chromaticity Diagram [165] [166].......................................................................

Figure 2. 14: Stopping power versus ion energy [143] ........................................................................66

Figure 3. 1: Block Diagram Illustrating the LOCC system and embedded modules [155]..................72

Figure 3. 2: Block Diagram of the LED Characterization Module ……...................................................73

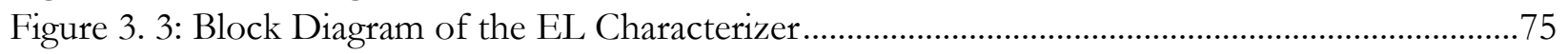

Figure 3. 4: Block Diagram of the Power Management module ..............................................................76

Figure 3. 5: Block Diagram of I2C isolated Module ...............................................................................78

Figure 3. 6: (From Left to Right) Chip Carrier and Chip Carrier Socket, Photodiode and LED PCB

modules (exploded), STF-1 CubeSat Rendering without Solar Panels ....................................................79

Figure 3. 7: Cross-section of connected LOCC system ...........................................................................8 80

Figure 3. 8: (Top Down View) LOCC Header Pin 1 for STF-1 connection .........................................81

Figure 3. 9: (Top Down View) LOCC Header pin 2 for STF-1 connection .........................................81

Figure 3. 10: (Top Down View) LOCC inter-board connection............................................................82

Figure 3. 11: Side profile of connection diagram for assembly of LOCC.............................................82

Figure 3. 12: Complete LOCC MCU/I-V Characterizer Circuit Diagram ............................................85

Figure 3. 13: LOCC MCU, the ATMEGA 2560-16AU ……..................................................................86

Figure 3. 14:Digital-to-Analog Converter connected to unity-gain OPAMP to source current to LEDs.

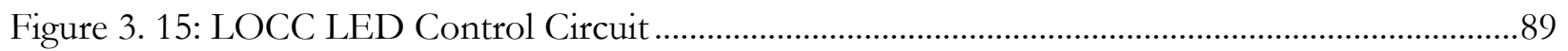

Figure 3. 16: LOCC Current/Power Monitor Circuit. ..........................................................................90

Figure 3. 17: Simplified LED Sourcing Circuit …...............................................................................

Figure 3. 18: EL Characterizer Complete Circuit...................................................................................93

Figure 3. 19: Single ADC-to-Photodiode Circuit.................................................................................. 94

Figure 3. 20: Logic Translation circuit of EL Characterizer....................................................................96

Figure 3. 21: LOCC I2C Isolation Module Circuit Diagram..................................................................97

Figure 3. 22: LOCC Power Management Circuit Diagram ..................................................................98

Figure 3. 23: Board Outline and dimensions of LOCC I-V Characterizer ......................................... 101

Figure 3. 24: (Left) Ground Plane layout, (Right) Designation between System Ground and Isolated

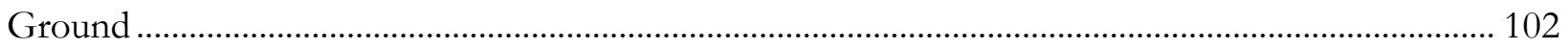

Figure 3. 25: (Left) Power Plane Layout, (Right) Designation between System 5V Power, Isolated 5V,

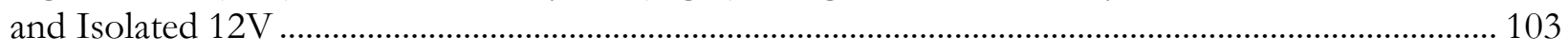

Figure 3. 26: (LEFT) Bottom Signal Plane Layout, (Right) Designation of modules and components 
Figure 3. 27: (Left) Top Signal Plane Layout, (Right) Designation of chip carrier socket components 104

Figure 3. 28: (Left) 3D View Bottom Side, (Right) 3D View Top Side.............................................. 105

Figure 3. 29: Board Outline and Dimensions of LOCC EL Characterizer......................................... 108

Figure 3. 30: LOCC EL Characterizer bottom side with outlined Isolated Ground Plane ............... 109

Figure 3. 31: LOCC EL Characterizer top side with outlined 3.3V and ground pours ..................... 109

Figure 3. 32: (Left) Bottom Signal with designated components ....................................................... 110

Figure 3. 33: (Left) EL Bottom Side 3D Rendering, (Right) EL Top Side 3D Rendering ................ 110

Figure 3. 34: (Top Down View) LOCC Header Pin 1 for STF-1 connection...................................... 111

Figure 3. 35:(Top Down View) LOCC Header pin 2 for STF-1 connection ...................................... 111

Figure 3. 36: Process flow diagram for experiments of LOCC system ................................................ 113

Figure 3. 37: Software Operation Flow Diagram............................................................................ 120

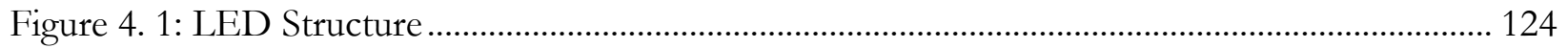

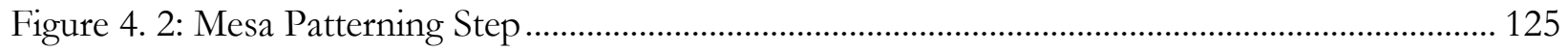

Figure 4. 3: Mesa Etch Processing and PR strip .............................................................................. 127

Figure 4. 4: Metal Patterning Process from photolithography.............................................................. 128

Figure 4. 5: Metal Evaporation and liftoff for p-contact ...................................................................... 128

Figure 4. 6: n-contact Patterning Process from photolithography …................................................... 129

Figure 4. 7: n-contact metal deposition and liftoff .............................................................................. 130

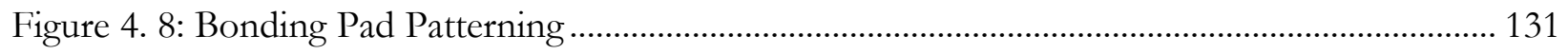

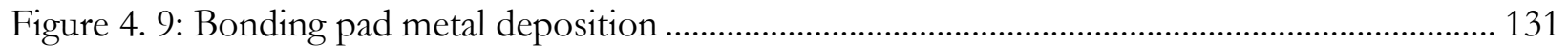

Figure 4. 10: 3D illustration of fabricated LED ................................................................................ 132

Figure 4. 11: Cut lines and dimensions for sample dicing ................................................................ 133

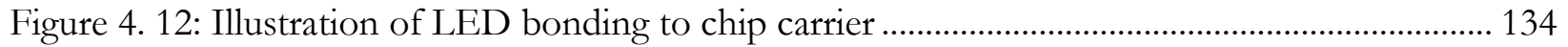

Figure 4. 13: Wire bonding diagram for LOCC compliance ................................................................ 135

Figure 4. 14: Recommended wire bond configuration....................................................................... 136

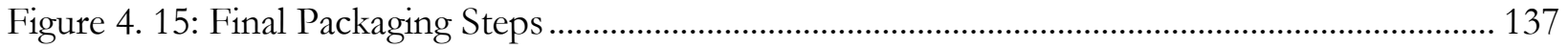

Figure 5. 1: (a)LED - Pre-package test, (b)Close-up of wire bonded LED, (c) Pre-encapsulation LED chip carriers, (d)Post-encapsulation chip carrier ................................................................................. 140

Figure 5. 2: (a)I-V Characterizer bottom side, (b)EL and I-V characterizer top side, (c)Functional test

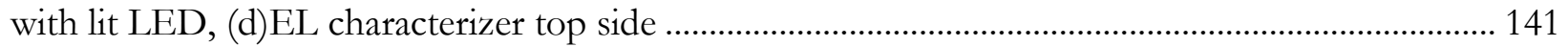

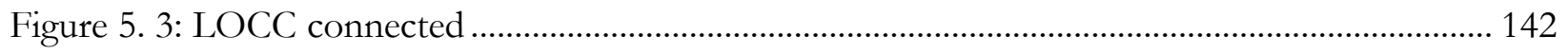

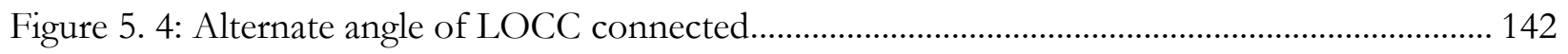

Figure 5. 5: I-V characterizer integration into CubeSat......................................................................... 143

Figure 5. 6: Full LOCC integration into CubeSat ............................................................................... 143

Figure 5. 7: Final assembly of the STF-1 CubeSat ........................................................................... 144

Figure 5. 8: I-V Characteristics of Chip Carrier 2. (a)Trial 1, (b)Trial 2, (c)Trial 3, (d) Trial 4......... 145

Figure 5. 9: Average and standard deviation I-V results of LEDs in chip carrier 2 ........................ 146

Figure 5. 10: (a)LED 5 - 4 trials, (b)LED 5 - Mean and standard deviation, (c)LED $6-4$ trials, (d)LED 6-Mean and standard deviation (e)LED 7 - 4 trials, (f)LED 7 - Mean and standard deviation 
Figure 5. 11: EL Characteristics of all LEDs. (a)Trial 1 - 24 LEDs, (b)Trial 1 - Zoomed Chromaticity values, (c) Trial 2 - 24 LEDs, (d) Trial 2 - Zoomed Chromaticity values (e)Trial 3 - 24 LEDs, (f)Trial 3 - Zoomed Chromaticity values, (g) Trial 4 - 24 LEDs ................................................................... 150

Figure 5. 12: Commercial Spectrometer Chromaticity outputs .......................................................... 151

Figure 5. 13:Box plot of light output over 4 trials. (a) LED 4, (b) LED 5, (c) LED 6, (d) LED 7 .. 153

Figure 5. 14: Light output intensity of LED chip carrier 1............................................................. 153

Figure 5. 15: Experiment 1 (a) Current consumed over time, (b) Average current consumed over time 155

Figure 5. 16: Experiment 2 (a) Current consumed over time, (b) Average current consumed over time 156 


\section{List of Tables}

Table 1. 1: Effects of Space Environment [102] ..................................................................................

Table 1. 2: Classification of small satellites by mass ................................................................................28

Table 2. 1: Damage Parameters and Used Equations.........................................................................69

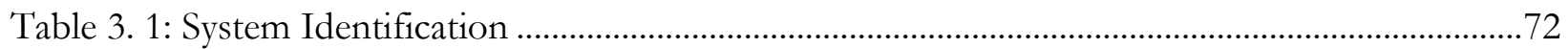

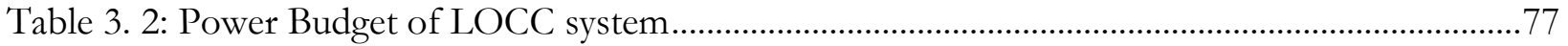

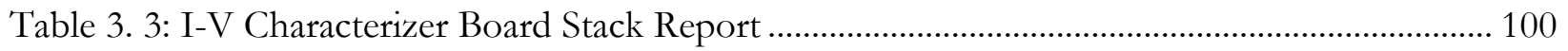

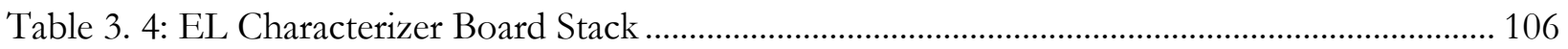

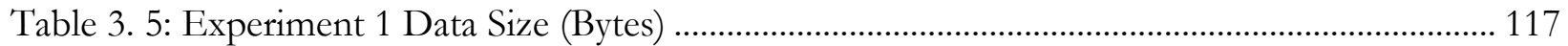

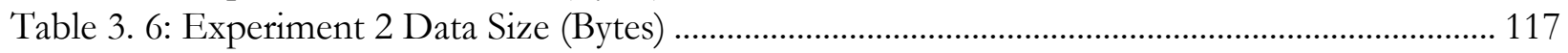

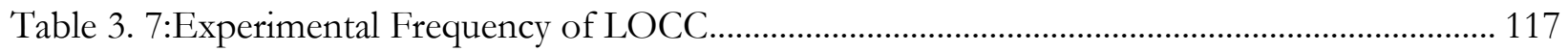

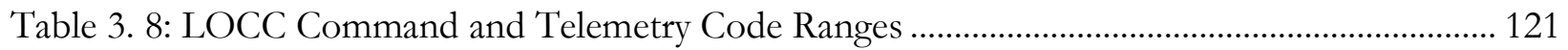

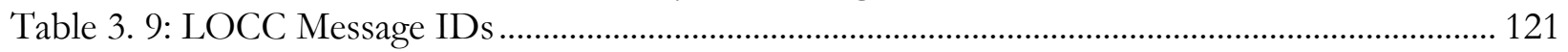

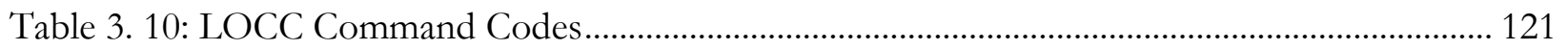

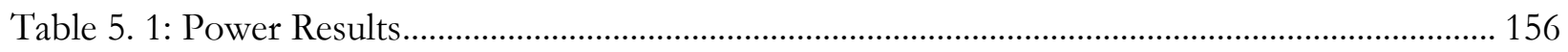

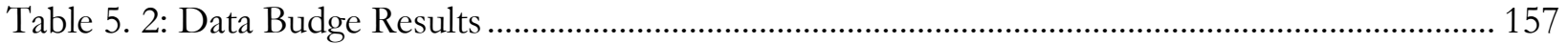




\section{Chapter 1: Introduction}

\subsection{Motivation and Objectives}

When developing optoelectronics for space applications, it is necessary to consider their operation in an environment with potentially high radiation levels and large temperature swings. To do this, a Low-powered Optoelectronic Characterizer for CubeSat (LOCC) has been developed. The motivation for this system is to provide a tool for evaluating the performance of III-V nitride based light emitting diodes (LEDs) and photodetectors (PDs) while in space. The LOCC platform was developed for West Virginia's first spacecraft, STF-1, which is a 3-unit CubeSat vehicle developed in a joint effort between West Virginia University (WVU) and NASA's Independent Verification and Validation (IV\&V).

\subsubsection{Problem Statement}

The cost for launching payloads into space can range from $\$ 10,000$ to $\$ 45,000$ per pound, depending on flight [2] [3] [4]. This means that weight significantly contributes to the cost of space missions. The optical and electrical properties of LEDs are subject to ionizing damages that cause degradation. This has led to the use of shielding on electronics, which increases the overall weight of the spacecraft [5] [6]. More shielding leads to increased overall weight, lower available payload capacity, and increased cost. To counter the additional weight from shielding, developing radiationhard optoelectronic devices allows for a reduction in the amount of shielding used, improves electronics reliability, and decreases spacecraft weight and associate launch costs. To validate approaches used to create radiation hardened devices, specifically LEDs, their optical and electrical properties must be examined while in the environment of space. 
CubeSats provide a low-cost testbed to observe the operation of LEDs while in space. CubeSats are $10 \mathrm{~cm} \times 10 \mathrm{~cm} \times 10 \mathrm{~cm}$ satellites that were originally standardized by California Polytechnic State University (Cal Poly) in 1999 [7]. CubeSat launches take advantage of ride-sharing opportunities that are offered with increasing frequency through collaborations between small satellite developers and rocket companies. For these launches, CubeSats are a secondary payload. Because of this, CubeSats can end up with an orbital altitude based on the mission of the primary payload, and undergo different effects such as atmospheric drag and ionospheric radiation depending on altitude and inclination. A CubeSat may be launched into a low Earth orbit (LEO) that will orbit between 160 $\mathrm{km}$ and 2,000 km [8]. These factors directly impact the lifetime of a CubeSat and its mission duration.

The goal of this work is to create and evaluate an optoelectronic characterization system that is compatible with the space and power requirements of a CubeSat platform that will measure the electrical and optical properties of III-V GaN based LEDs while in the space environment. This platform will enable the evaluation of radiation hard optoelectronics, as well as the reliability assessment of minimally shielded devices. This work aims to reinforce the foundation and advancement of optoelectronics and semiconductor devices for applications in the space environment.

\subsubsection{Motivation}

LEDs have a broad range of applications and are used globally as efficient sources of light. The solid-state lighting (SSL) industry today is motivated by the low energy consumption, control, color tuning capability, aesthetics, and long lifetimes of LEDs, making them desirable replacements for traditional light sources (incandescent bulbs, fluorescent lamps, etc.). These factors tie together in a national and global market that is dedicated to high efficiency and reduced energy consumption. The U.S. Department of Energy (DOE) has projected that LEDs will make up 30\% of U.S. lighting installations by the year 2020. They also forecast that, by 2035, 5.1 quadrillion Btus (quads) in annual 
energy consumption will be cut. This is equivalent to the total annual energy consumed by 45 million U.S. homes. Between the years of 2015 and 2035, this comes close to $\$ 630$ billion in savings towards energy costs [63]. NASA has even sponsored research efforts evaluating the use of LEDs to promote astronauts' health while in space [64]. LEDs are a crucial part of developing technologies, and will continue to be influential globally and as part of developing space systems.

In 1971, one of the first missions to implement optoelectronic navigation system was the Mariner IX mission to Mars [69]. At the heart of this system are the optoelectronic devices (LEDs and PDs). The mission focused on imaging and optical measurements of celestial bodies (stars, moons, planets, comets, and asteroids). When examining these large bodies, optical navigation determined where the image should appear regarding the spacecraft, actual position of the target body, and utilized image processing to determine the coordinates of the target object.

The Mars Reconnaissance Orbiter (MRO) was launched in 2006, built by Lockheed Martin, and supervised by NASA Jet Propulsion Laboratory (JPL). This satellite utilized several different optical instruments such as a spectrometer, visible spectrum cameras, and radar [70].

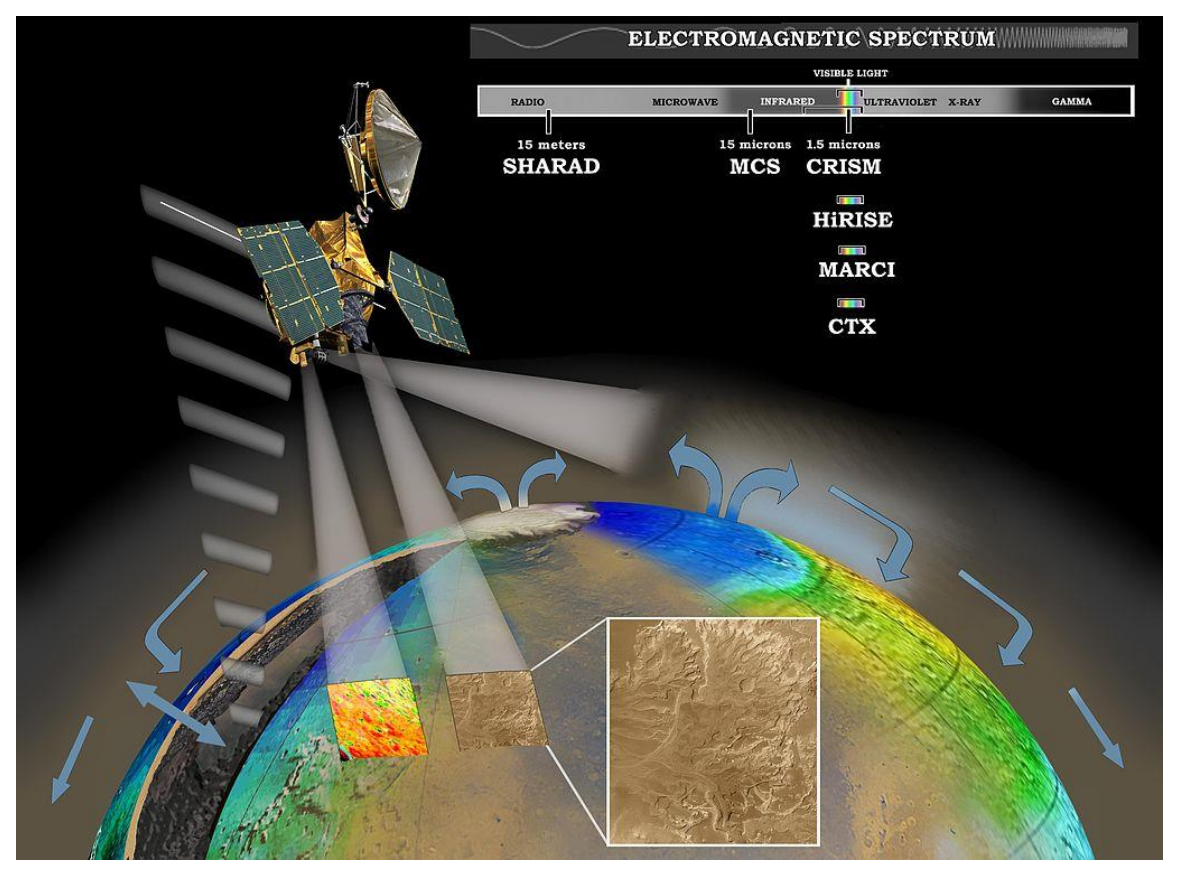




\section{Figure 1. 1: Illustration of MRO instrumentation [71]}

Among these instruments were the High-Resolution Imaging Science Experiment (HiRISE) camera that collected images in blue-green, red, and near infrared (NIR) bands [72]. Also, a Context Camera (CTX) that provided greyscale images and utilized a Maksutov Cassegrain telescope with a 5,064-pixel array Charged Coupled Device (CCD) camera [73]. The MRO also housed the Mars Color Imager (MARCI) that took images of Mars in five visible spectrums and two ultraviolet bands [74]. The MRO housed the Compact Reconnaissance Imaging Spectrometer for Mars (CRISM) that measured 544 channels from visible and near infrared bands (VNIR). MRO also carried a Mars Climate Sounder (MCS) with multiple VNIR and far infrared (FIR) channels. The MCS measured weather temperature, pressure, humidity, and dust [76]. These devices and systems led to the discovery of water ice in ice cap measurements, ice exposure in craters, chloride deposits, and even photographed avalanches [77] [78] [79] [80].
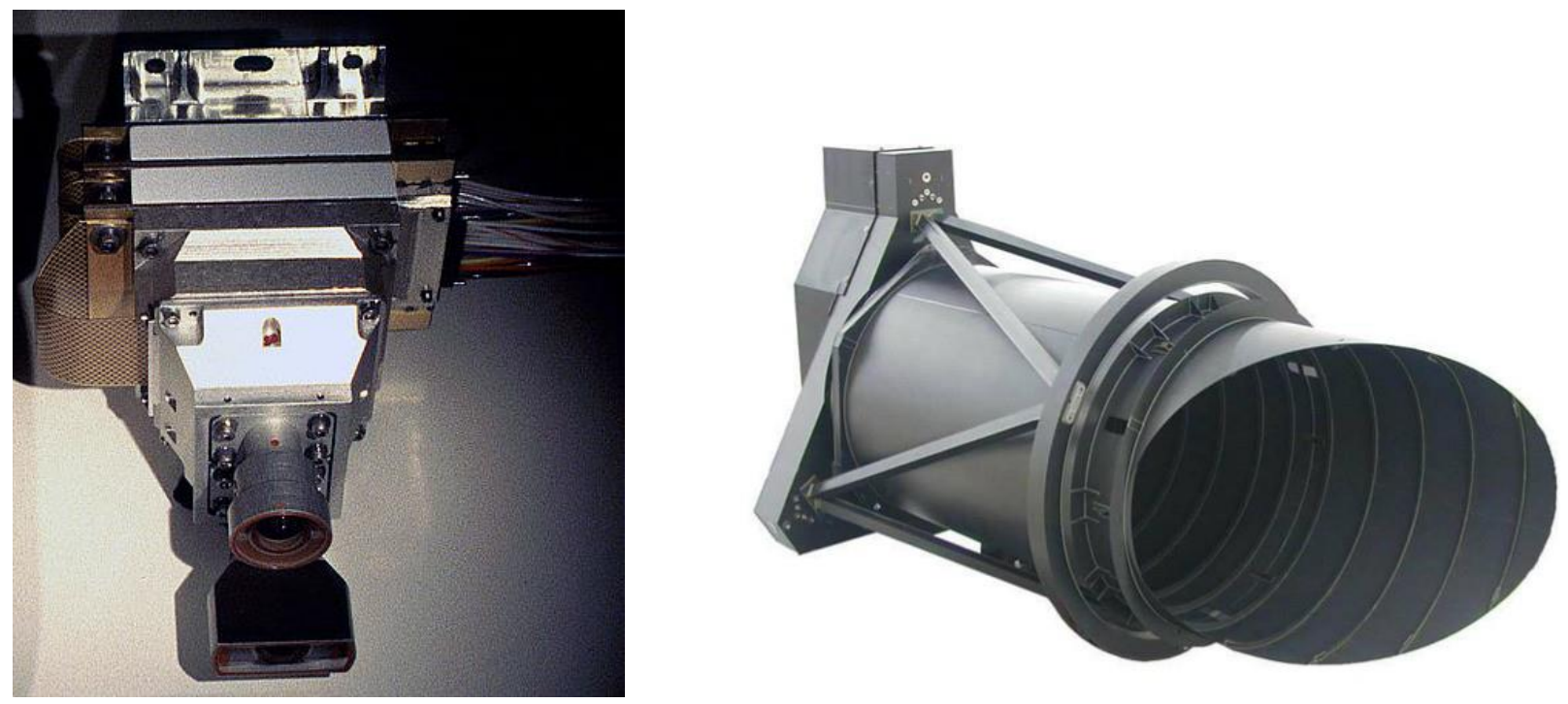

Figure 1.1.2a: Mars Color Imager [81], Figure 1.1.2b: HiRISE Telescope Camera, Figure 1. 2: (LEFT)Mars Color Imager, (RIGHT) HiRISE Telescope Camera [82] 
Automated Rendezvous and Docking/Capture sensors have been examined as another means of utilizing LEDs in space applications [89]. Visible and infrared electromagnetic (EM) spectrumbased sensors and LEDs can be used in the creation of light detection and ranging (LIDAR) systems [91]. SpaceX's Dragon utilizes IR camera and image processing to provide the relative data on navigation range [92]. NASA is currently developing an International Docking Adapter (IDA) that will allow the International Space Station (ISS) to dock with any target. Figure 1.3 illustrates the use of NASA/Boeing's Pressurized Mating Adapter (PMAs) that utilize reflective surfaces and LIDAR system for automated docking procedures.

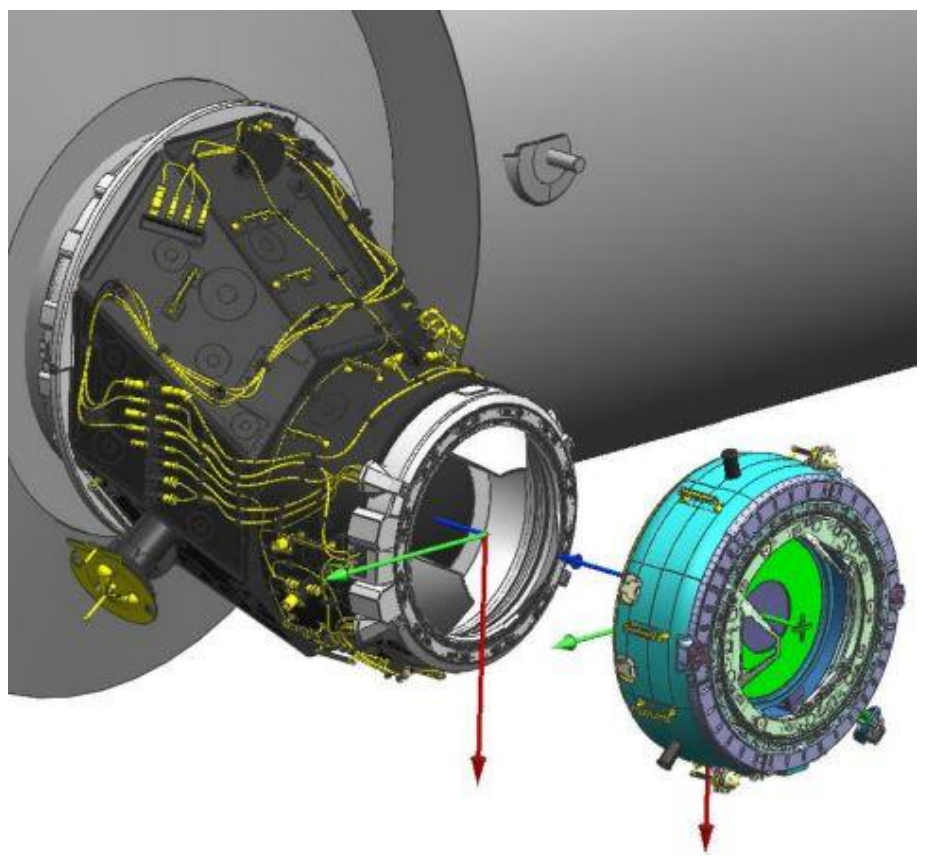

Figure 1. 3: ISS ID $A$ to PMA interface [89]

\subsubsection{Optoelectronics in Small Satellite Applications}

The technologies onboard the MRO, Mariner, and other large-scale systems do not fall within the constraints of an academic budget, so CubeSat missions offer similar technologies when performing celestial observations. The effort described in [75] examines the capabilities of CubeSats 
for Earth observation. Like the largescale satellites, CubeSats are being equipped with CCD cameras, radio receivers, spectral measurements lens, and antennas for examining conditions of planets and space. CubeSat missions have performed Earth observations regarding NIR and visible bands and disaster monitoring [83] [84] [85], showing that CubeSat imaging can rival that of larger satellites.

A CubeSat system has been developed to test in-orbit technologies that are necessary for autonomous assembled and docking for a multiple mirror micro-satellite array [93]. The Autonomous Assemble of a Reconfigurable Space Telescope, or AAReST, has been developed to verify and improve autonomous assembly. The AAReST consists of three $3 \mathrm{U}$ CubeSats that utilizes LIDAR/Camera systems as well as other basic CubeSat components such as Battery, GPS, and Transceivers. The mission for the AAReST was developed by California Institute of Technology (CalTech) and University of Surrey-Surrey Space Centre (SSC). The overall mission is to develop a self-supporting system for autonomy. Figure 1.4 illustrates the layout of the AAReST configuration.

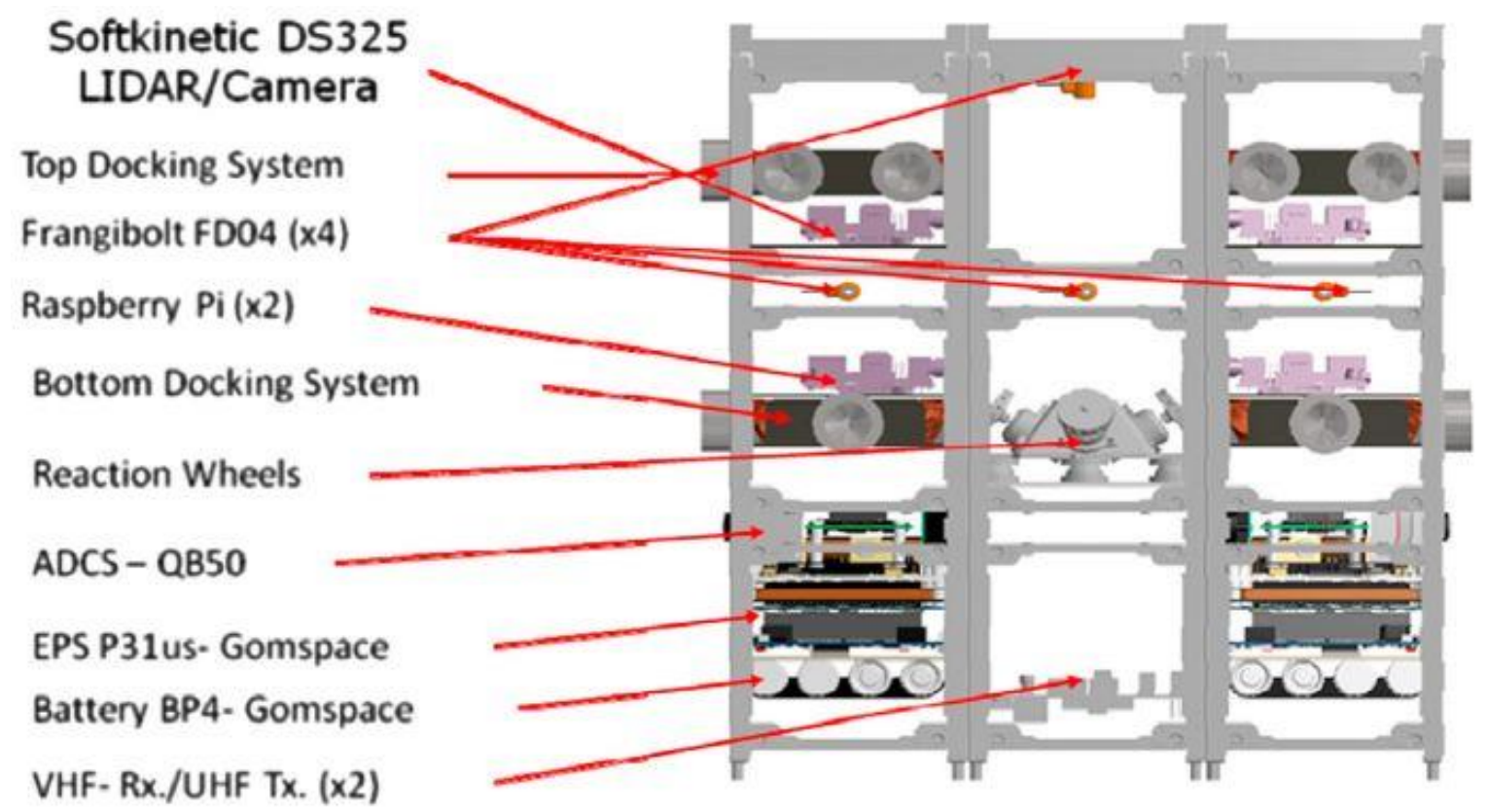

Figure 1. 4: AAReST CubeSat System [93] 
The examples presented above have a common factor; the reliable operation of optoelectronic devices. Coupling the growing need for space ready solar panels, LEDs, and photodetectors, it is seen that reliable electronics in the space are important. To explore space, reliable optoelectronics are necessary in the development of space technologies. It is important to stress the need for electronics that must be able to withstand the space to assure successful missions [68].

As can be seen in the preceding examples, dependable optoelectronic devices are key components to the successful operation of spacecraft missions. To improve these devices, it is necessary to develop analytical tools that can be utilized in their native environment. This thesis focuses on the design and implementation of hardware systems that analyze the operation and characteristics of minimally shielded III-V Nitride based LEDs while on orbit. The concept of operations of this work is aided by an understanding of small satellites, specifically CubeSats, an understanding of LEDs, and the motivation of selected materials versus ionizing radiation. These topics will be introduced in the following sections.

\subsection{Overview of Electronics in Space Applications}

The space environment poses many challenges to conventional semiconductor devices. Highenergy protons, electrons, and ions that are found in low Earth orbit (LEO) can wreak havoc on electronic devices while in space [12]. It is important to understand the general effects of space on electronics

\subsubsection{Earth's Atmosphere}

Understanding the effects of irradiation on electronics is paramount when considering methods to reduce shielding and increase the lifetime of semiconductor devices (Si-based, III-V, etc.) while on orbit. It is necessary to see how space weather will effect our elecontrics. To do this, an 
overview of Earth's atmosphere is provided to illustrate the different layers of the atmosphere and how they are related. Figure 1.5 shows the break down of atmopsheric layers.

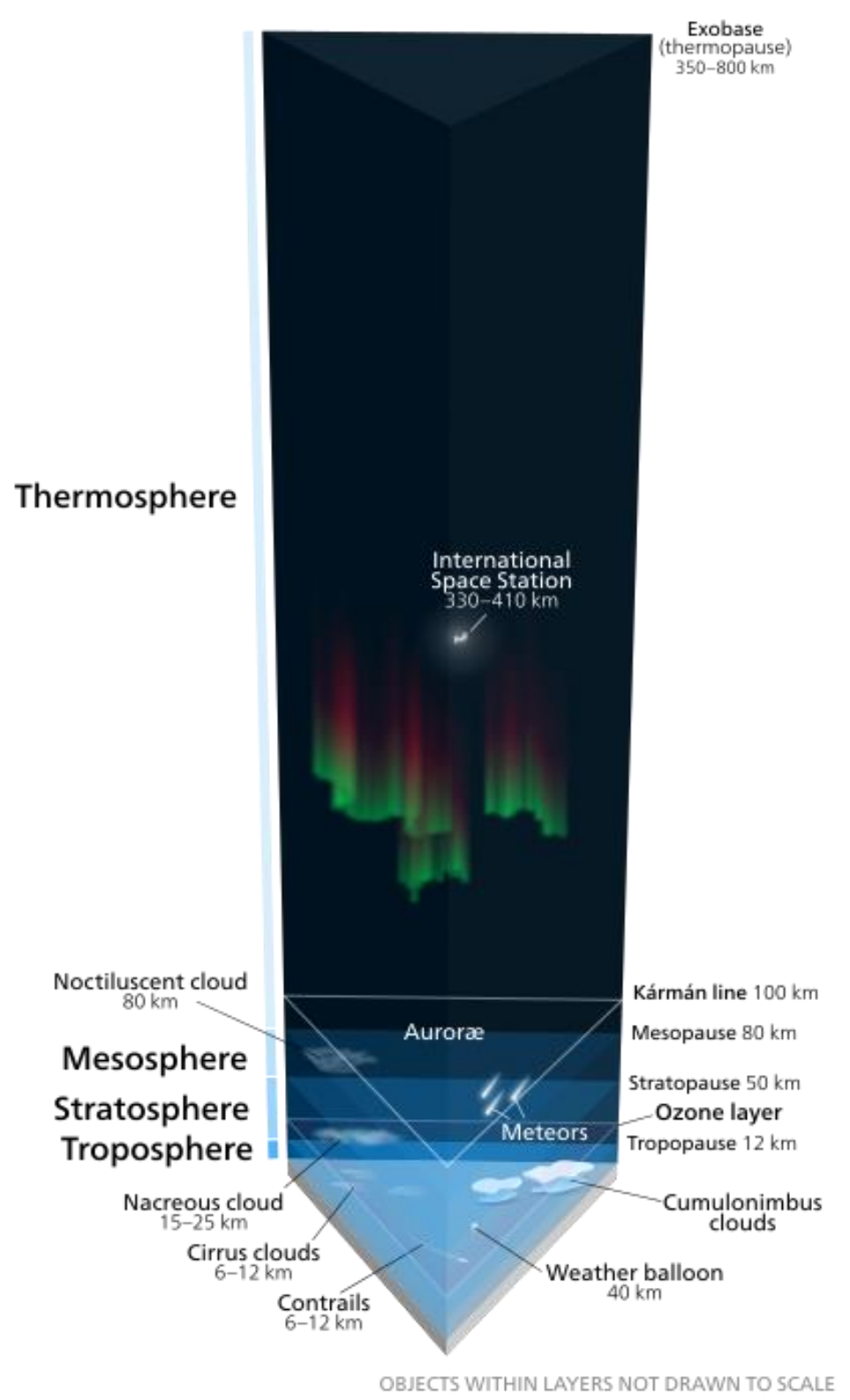

Figure 1. 5: Illustration of Earth's atmospheric boundaries [94]

Starting from the Earth's surface, the troposphere extends to about $12 \mathrm{~km}$. This varies depending relatively between the poles and equator [95]. The stratosphere sits atop the Tropopause, which makes 
up a $43 \mathrm{~km}$ cushion. Temeperatures here range from $-60^{\circ} \mathrm{C}$ to $0^{\circ} \mathrm{C}$. This is due to the absorption of ultraviolet radiation from the Sun [96]. The Mesosphere extends above the Stratosphere another 50 $\mathrm{km}$ into the mesopause at an additional $35 \mathrm{~km}$ [97]. Temperatures in this part of the atmosphere can reach an average $-85^{\circ} \mathrm{C}$, and it is considered the coldest place on Earth [98]. The Thermosphere exists from $80 \mathrm{~km}$ to $1000 \mathrm{~km}$. This height can be varied due to the influence of solar activity [99]. It is within this sphere that STF-1 will orbit. More specifically, in the thermosphere's lower range. The exosphere is Earth's outermost layer and extends to $10,000 \mathrm{~km}$ where the effects of solar wind take place. This thesis focuses on the effects of the lower thermosphere.

Table 1. 1: Effects of Space Environment [102]

\begin{tabular}{|c|c|}
\hline Environment & Main effect on space systems \\
\hline $\begin{array}{l}\text { Cosmic rays }(\sim 100 \mathrm{MeV} \text { to } \sim 1 \mathrm{GeV}) \\
\text { High-energy solar particles } \\
\quad(\sim 100 \mathrm{keV} \text { to } \sim 300 \mathrm{MeV} \text { (sometimes } \mathrm{GeV}))\end{array}$ & $\begin{array}{l}\text { Ionizing and non-ionizing dose effects } \\
\text { (components and material degradation, living } \\
\text { cell damage) } \\
\text { Single-event effects }\end{array}$ \\
\hline $\begin{array}{l}\text { Radiation belt particles } \\
\text { (electrons: } \sim 100 \mathrm{keV} \text { to } \sim 10 \mathrm{MeV} \text {; } \\
\text { protons: } \sim 1 \mathrm{MeV} \text { to } \sim 1 \mathrm{GeV} \text { ) }\end{array}$ & $\begin{array}{l}\text { Ionizing and non-ionizing dose effects } \\
\text { (components and material degradation, living } \\
\text { cell damage) } \\
\text { Single-event effects } \\
\text { Deep dielectric charging }\end{array}$ \\
\hline $\begin{array}{l}\text { Ionospheric plasma }(\sim 0.1 \mathrm{eV}) \\
\text { Plasmasphere }(\sim 1 \mathrm{eV})\end{array}$ & $\begin{array}{l}\text { Particle flux effect (electrical current noise, } \\
\text { erosion, sputtering) }\end{array}$ \\
\hline $\begin{array}{l}\text { Solar wind plasma } \\
\quad \text { (electron: } \sim 1 \mathrm{eV} \text {; proton: } \sim 0.1 \text { to } 1 \mathrm{keV} \text { ) }\end{array}$ & Small electrostatic potential \\
\hline $\begin{array}{l}\text { Auroral filament plasma }(\sim 100-10 \mathrm{keV}) \\
\text { Plasmasheet plasma }(\sim 1 \text { to } 10 \mathrm{keV})\end{array}$ & $\begin{array}{l}\text { Particle flux effect (electrical current noise, } \\
\text { erosion, sputtering) } \\
\text { Strong electrostatic potential }\end{array}$ \\
\hline Visible and IR photons & Thermal \\
\hline $\mathrm{UV}, \mathrm{X}$ and $\gamma$ photons & Ionizing effects \\
\hline Radio waves & EM noise \\
\hline Geomagnetic field & $\begin{array}{l}\text { Magnetic torque on current loop } \\
\text { Induced electric field in conductors }\end{array}$ \\
\hline Interplanetary magnetic field & $\begin{array}{l}\text { Torque } \\
\text { Induced electric field }\end{array}$ \\
\hline Neutral atoms & $\begin{array}{l}\text { Spacecraft drag } \\
\text { Chemical reactions on surfaces, and } \\
\text { associated degradation }\end{array}$ \\
\hline Microparticles & $\begin{array}{l}\text { Puncture } \\
\text { Transient dusty plasma cloud }\end{array}$ \\
\hline
\end{tabular}

Table 1.1 describes the effects of space. Electronics undergo effects caused by cosmic rays, high energy solar particles, radiation belt particles, Ionospheric Plasmas, visible and IR photons as well as UV, X- 
Ray, and gamma photons. Electronics engieeers must be wary of thermodynamic and electromagentic effects, and should consider appropriate protection methods, which can influence the weight and cost of the component and system. The diveresity of the electromagnetic and thermal environments on electronics have had an impact on the way electronics are developed for space [103].

\subsubsection{Ionosphere}

Between $60 \mathrm{~km}$ and 1,000 km, the ionosphere stretches across the thermosphere, mesosphere and exospehers. The Sun's ionizing partlicles reach this region, and play a crucial role in the electrical flow of the atmosphere, forming the edge of the magnetosphere [100]. At this height in the atmosphere, wavelengths in the ultraviolet (UV) and x-ray, or shorter wavelengths, can have an ionzing effect on the gas atoms and molecules that exist here. Ionization is heavily influenced by solar radiation and galactic cosmic radiation (GCR). Two major phenomon appear from the effects of the Sun: particle precipitation and convection of ionospheric plasma. These ionization effects are illustrated in figure 1.6. 


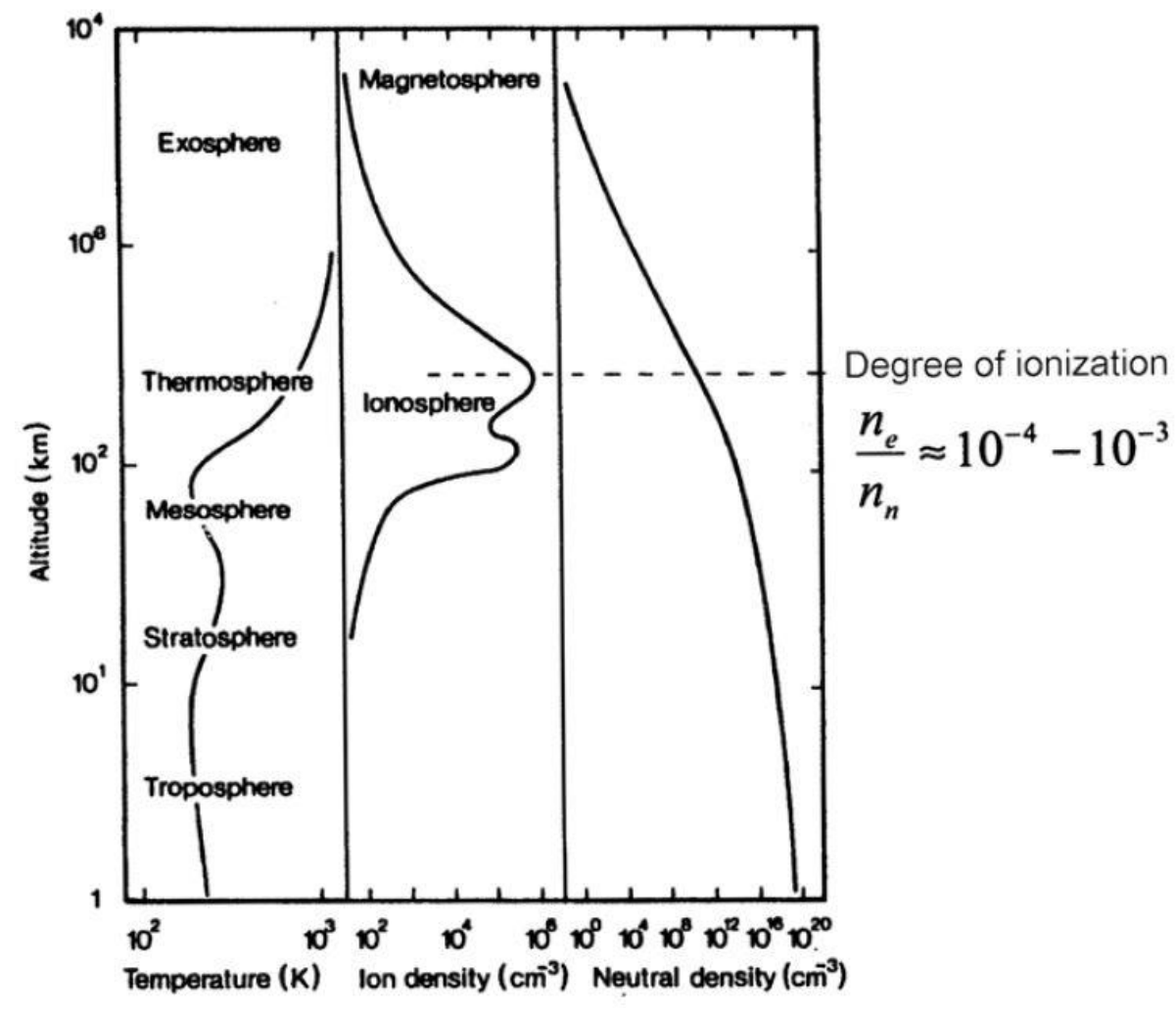

Figure 1. 6: Basic Quantities of Ionosphere as function of height [101]

\subsubsection{Radiation Effects on Solid-State Electronics}

The space environment and its high-energy particles can produce damage in electronics. These effects are listed below [14]:

- Total Ionizing Dose (TID) - Cumulative dose damage

- Enhanced Low Dose Rate Effects (ELDRS) - Low doses produce disproportionate damage in higher ranges

- Neutron and Proton Displacement

- Single Event Effects (SEE) - Device disruption by ionic charge 
TID is caused by displacements in the lattice that come from ionzing radiations of high-energy XRays, gamma rays, or charged particles such as protons. Displacement of atoms due to nuclear interactions in lattice structures is widely observed. The displaced lattice atom can produce vacancies, interstrials and complexities hereafter. This may result in decreased electron mobility, voltage shifts, decreases in transconductance, and decreases in saturation currents [15]. In LEDs, this can result in reduced light output with increase in currents. Figure 1.7 illustrates atomic damage in a crystal structure of electronics.

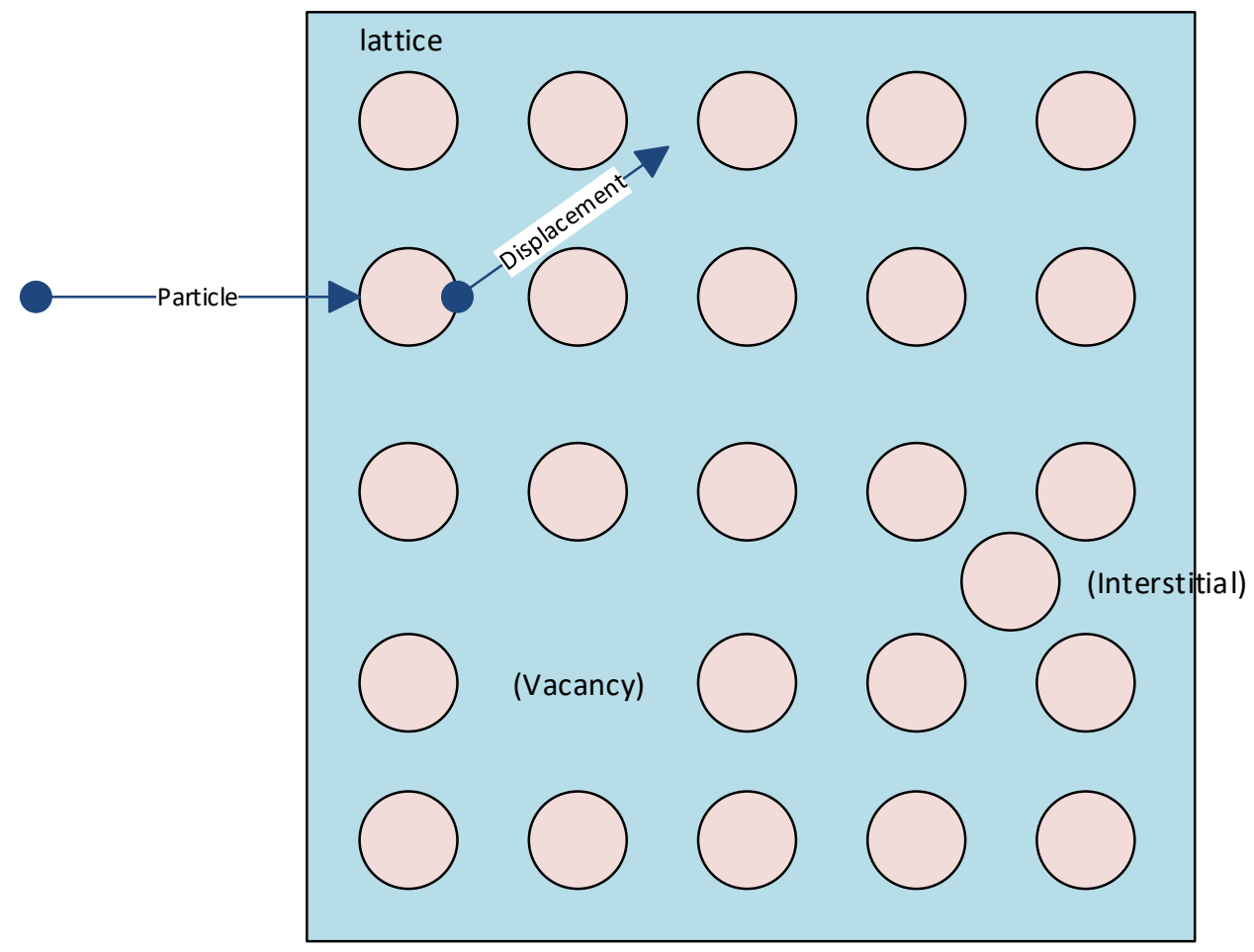

Figure 1. 7: Atomic Displacement and point defects (vacancy and interstitial)

Protons have shown to produce more point defects, and have the largest effect on electronics due to electromagnetic interactions within the crystal that are dependent on particle energy and type [16] [17] [18]. Radiation effects can act as generation-recombination or trapping centers for charged 
carries [19]. This has effects on voltage depletion levels, reverse currents, and signal levels. To examine these effects in the lattice, the damage is scaled using nonionzing energy loss (NIEL) [20]. This scaling allows the macroscopic electrical properties to be compared among devices. It is performed on the energy spectra of the particles of a radiation field with the appropriate NIEL factor to express the fluence in terms of a reference monochromatic particle [21]. The defects of space charge density $\left(N_{e f f}\right)$ can be summarized as a function of fluence. Figure 1.8 describes the absolute value of voltage for varying thicknesses and fluence of protons; describing decay along with an increase in space charge after type inversion.

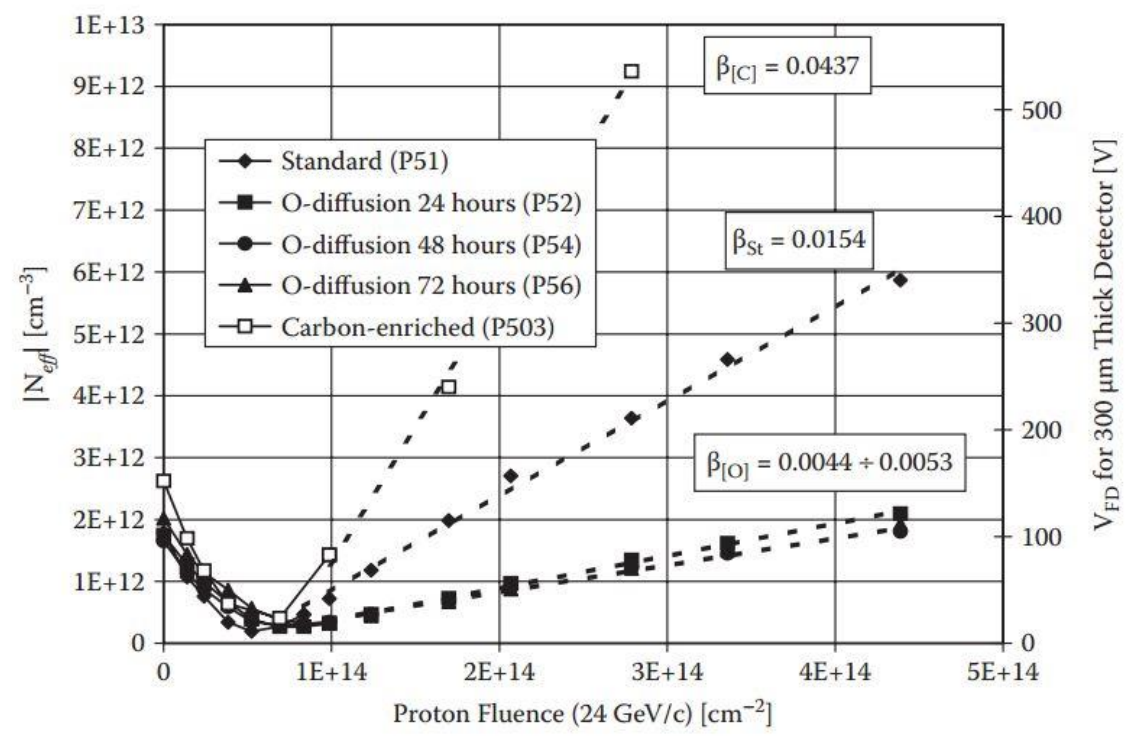

Figure 1. 8: Space Charge and Forward voltage as function of proton fluence [22]

Similiarly, the effects of irradiation in the electical characteristics of silicon photodetectors with varying thickness of microstriping is seen in Figure 1.9. 

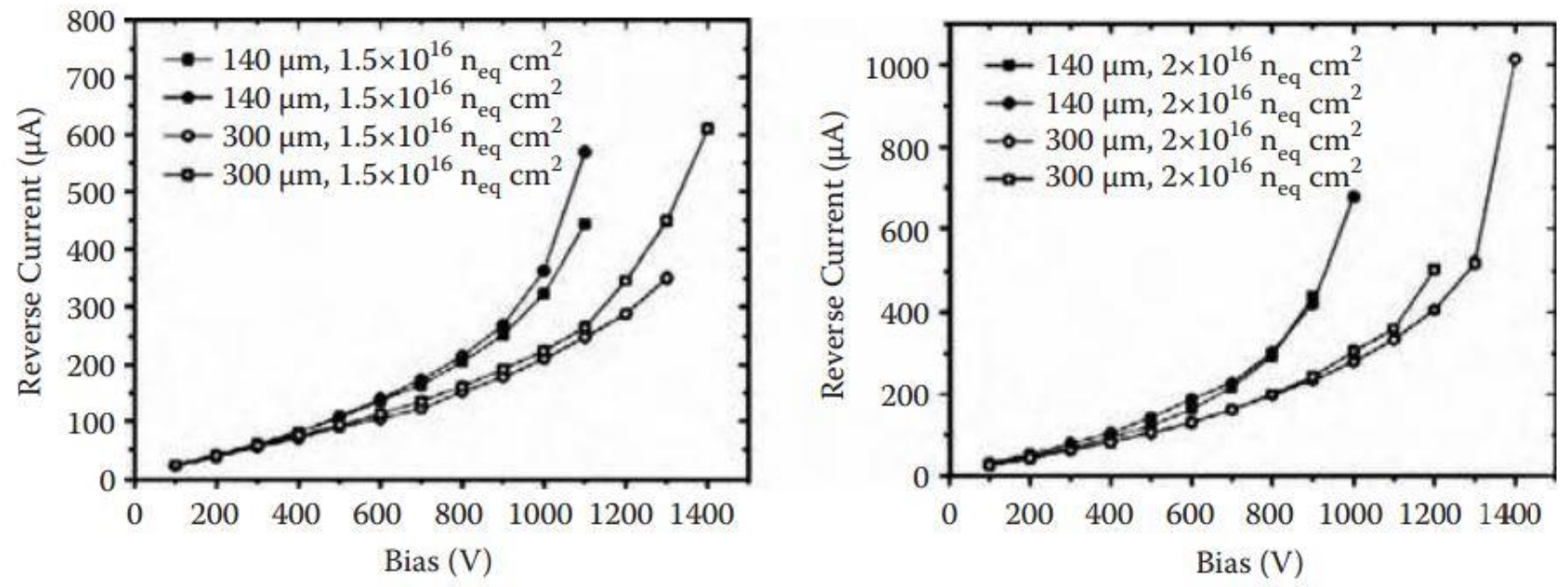

Figure 1. 9: Reverse Current as versus bias voltage with varying thicknesses and irradiation [21]

High-energy electrons also casue significant damage to silicon electronics. Leakage current, measured in terms of volume density increase, have been observed [23]. Figure 1.10 shows the linear fit of multiple silicon materials after electron irradiation and 8 minutes of annealing at $80{ }^{\circ} \mathrm{C}$.

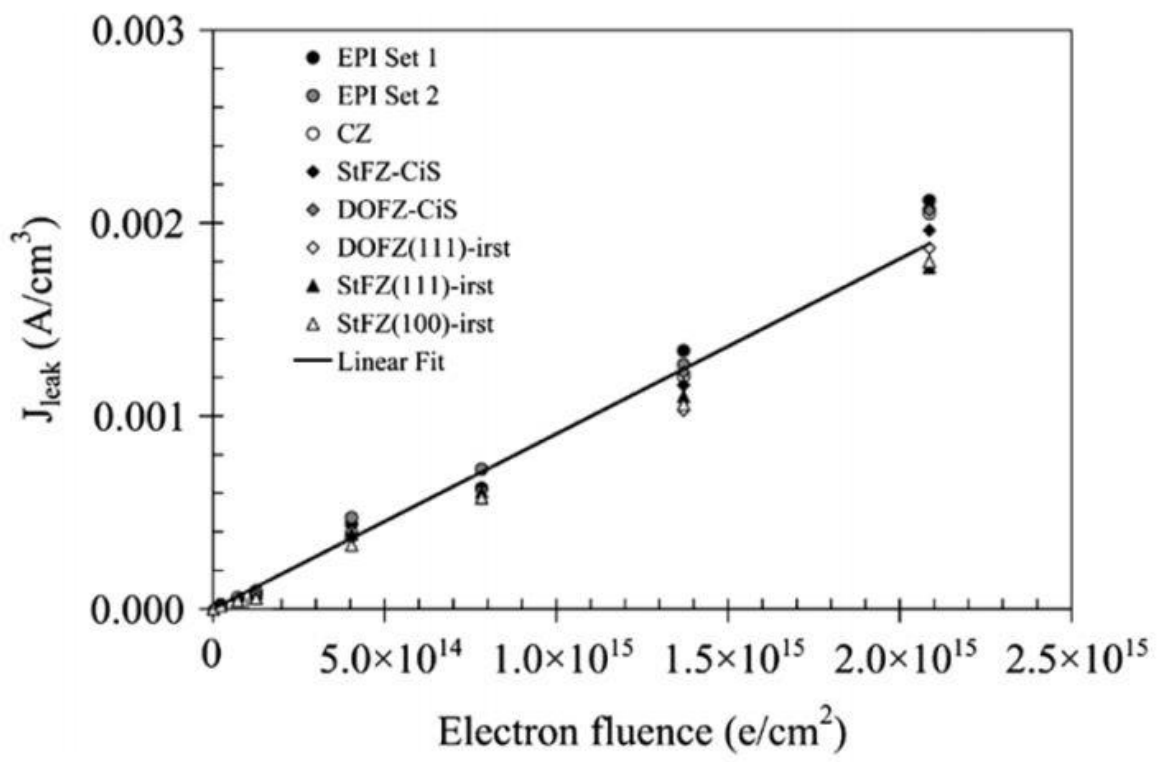

Figure 1. 10: linear fit of multiple silicon materials after electron irradiation after 8 minutes of annealing at $80{ }^{\circ} \mathrm{C}$ [23] 
The effects of radiation can also be seen within transistors as a decrease in current gain. Figure 1.11 describes emitter current density decreases pre- and post-proton irradiation [24].
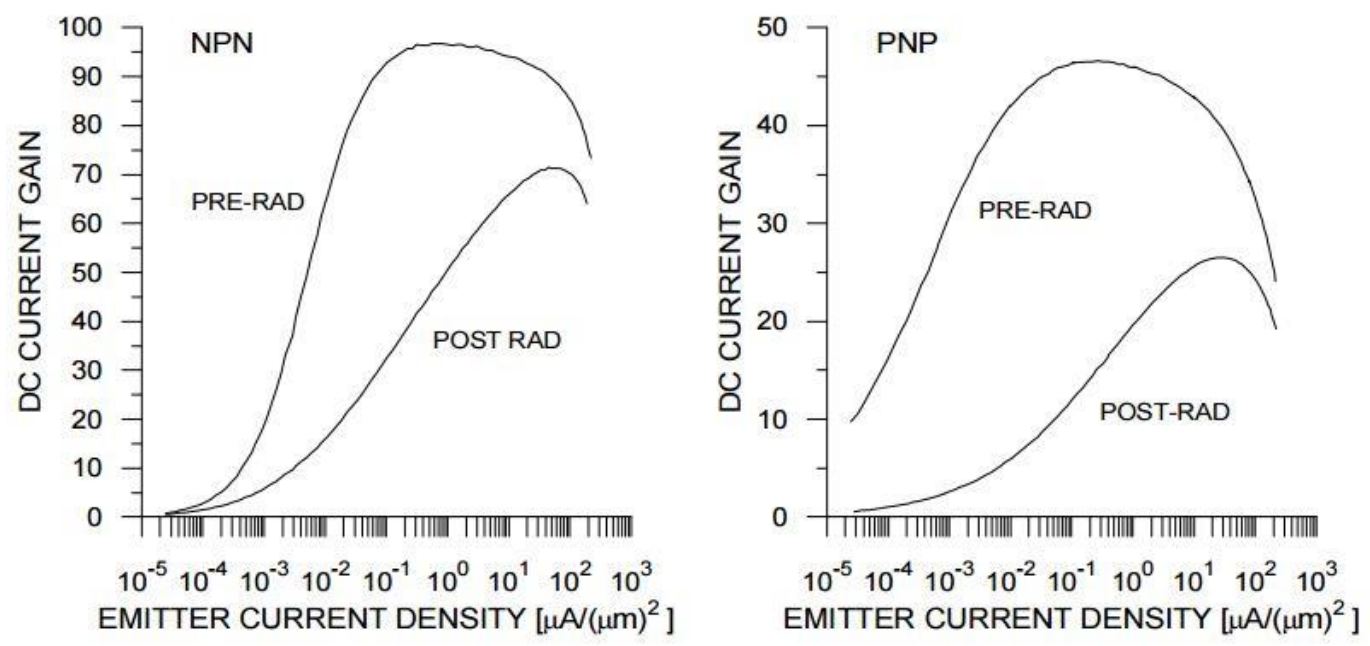

Figure 1. 11: DC Current Gain of npn and pnp transistors [24]

It is easy to see that irradiation of the devices has significant effects on device characteristics. These characteristics play a key role in crafting stronger radiation hardness in electronics. With these effects in mind, progress needs to be made in the development of robust semicondutor devices which can withstandprolonged exposure to the effects of space.

\subsubsection{Temperature Effects on Electronics}

It is important to note the effects of temperature while in space, where temperatures can range from $-193{ }^{\circ} \mathrm{C}$ to $+250{ }^{\circ} \mathrm{C}[25]$. The requirments of electronics for space and deep space operations need to be capable of operation within these ranges and exhibit long lifetimes while under harsh conditions. Temperature can have detrimental effects on devices such as photosensors and transistors. 
Studies are ongoing at the NASA Glenn Research Center in efforts of designing more temperaturerobust circuits and devices to operatre over greater temperature extremes. The goal is to develop devices and technology for low- and high-temperature operation. Figure 1.12 illustrates temperature effects on diodes and transistors. Effects on transistors show shifted gate voltage and a diminished saturation current.
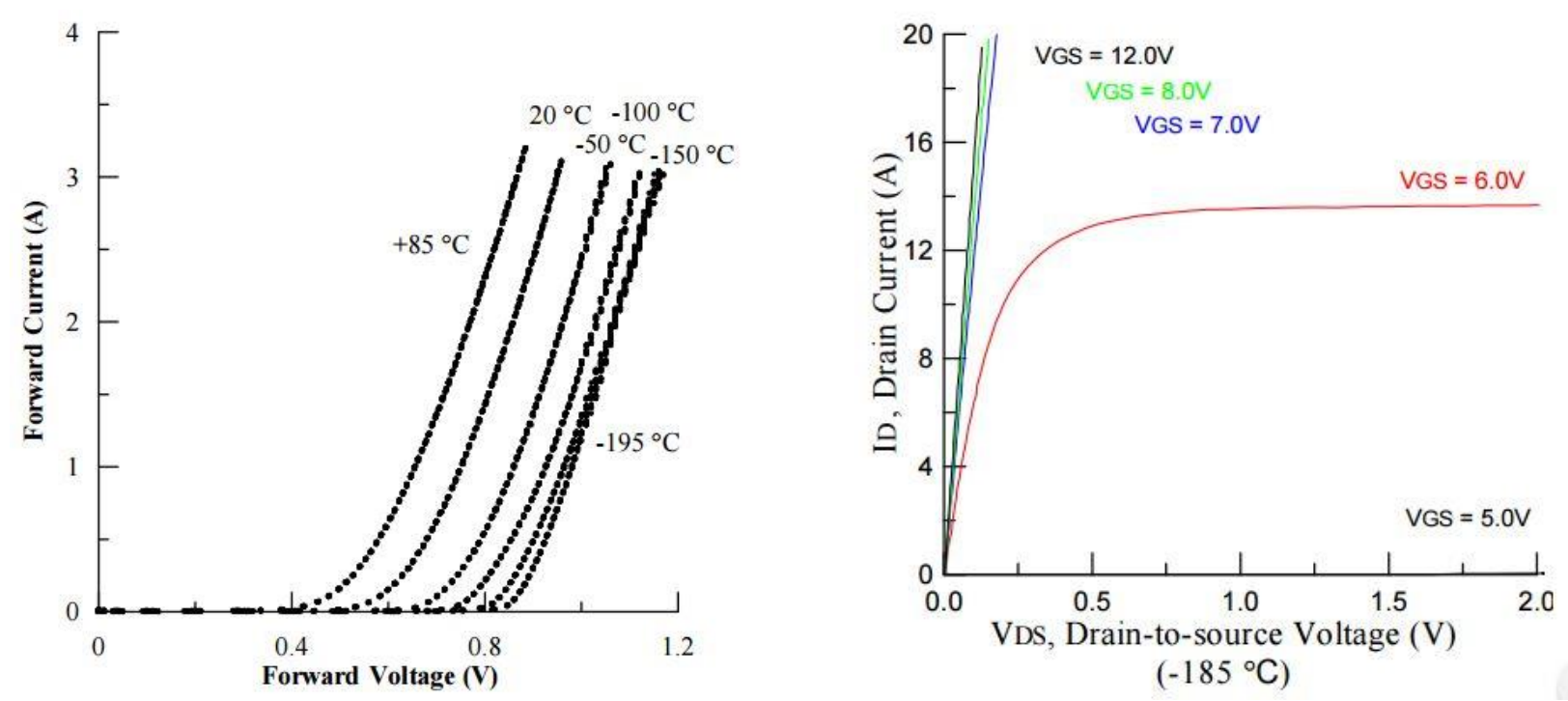

Figure 1. 12: (LEFT) I-V Characteristics of SiGe diode as a function of temperature, (RIGHT) I-V characteristics of Si MOSFETs as functions of temperature [25]

Working towards reliable devices for space poses a large challenge for the continued exploration of space. Providing tools that can enable these developments are paramount for the success of space exploration.

\subsubsection{Hardness-by-Design}

It is important to note here the work that has been done towards the hardening of device technology to fight against irradiation. Techniques have been adopted and are currently catagorized 
as Hardness-by-Design. Extensive research has been performed towards the hardning of CMOS circuits and system-on-chip architectures [49 - 55]. In CMOS research, techniques such as guard rings and width/length ratio control can lead to radiation-tolerent devices. There has been performance metrics for devices such as charge coupled devices (CCDs), charge-injection devices (CIDs), and active-pixel sensors (APS) that have been examined for imaging while in space. The use of MOSFET technology, coupled with hardness-by-design devices, allows for the advancement of imaging systems that produce higher quality and less interferance. This will prove important for upcoming missions such as the James Webb Space Telescope (JWST) [56]. Degredation in photodetectors is a leading interest in space-technologies [57]. It has been shown that the sensitivity of photodetector becomes compromised while in space [58]. Research regarding the hardening of optoelectronics have suggested that the thinning of layers in the material stack offers an increased defense against radiation dosing. The work presented in [59] has shown that layers of Indium Tin Oxide (ITO) are effective with conductivity and stable while irradiated. The evaluation of passivation layers, epitaxial growth techniques, or photonic cystals may help in the prevention of TID effects and even enchance ligh output in devices. Consideration of redundancy and guarding structures may also prove beneficial in the improvement of LEDs and optoelectronics. The improvement of CCDs, CIDs, APSs, LEDs, and lasers are a byproduct of design and materials. Combining the natural hardness of $\mathrm{GaN}$ along with the robustness provided by hardness-by-design techniques provides a path towards reliable operation of devices in the space environment.

\subsection{Overview of GaN Materials for Space Applications}

Wide-bandgap III-V nitrides are shown to be radiation tolerent due to their inherint bond strength as binary and terenary compounds [26]. The measures of bond strength were previously determined to be inversely proportional to the volume of the unit cell [28]. When compared to GaAs, 
reports show GaN breakdown occurred two orders of magnitude higher [27]. These intrinic properties indicate that that GaN optoelectronics may be better for space applications. To reinforce this, it is important that unknown effects of space on GaN optoelectronics be examined.

GaN in LEO will be subjected to fluxes of high energy electrons, protons, gamma radiation, and neutron radiation. Proton and electron irradiation leads to primary defects in the form of vacancies and interstitials. This leads to secondary effects such as grain broundies, cracks, and voids [30] [31] [32]. It is currently unclear how gamma radiation effects GaN. as multiple reports have conflicting results. Statements have been made that gamma irradiation increases electron mobility, thus increasing drain currents [33] [34], while others have reported a decrease in drain current [35] [36] [37].

\subsubsection{Radiation Damage in GaN LEDs}

$\mathrm{GaN}$ is a promising material for space application electronics. This is due to the required displacement energy needed to move atoms from the lattice. The displacement energy is inversely proportional to the lattice constant and rivals materials such as GaAs[38]. It is important to understand the effects of irradiating GaN materials and what conclusions have been drawn. The most prominent damages and effects to GaN materials are summarzed herin.

Proton Damage - Damage caused by protons is dependent on particle energies and Rutherford Scattering. High-energy protons can penetrate packaging materials and affect the LEDs. The effects of proton irradiation have been studied in [39] and [40]. It has been found that III-V nitrides posses a superior hardness to proton damages, withstanding upwards of $115 \mathrm{MeV}$. Figure 1.13 shows irradiation effects normalized to their parameters. Detremental effects occur after $1 \mathrm{MeV}$ energy [41]. 


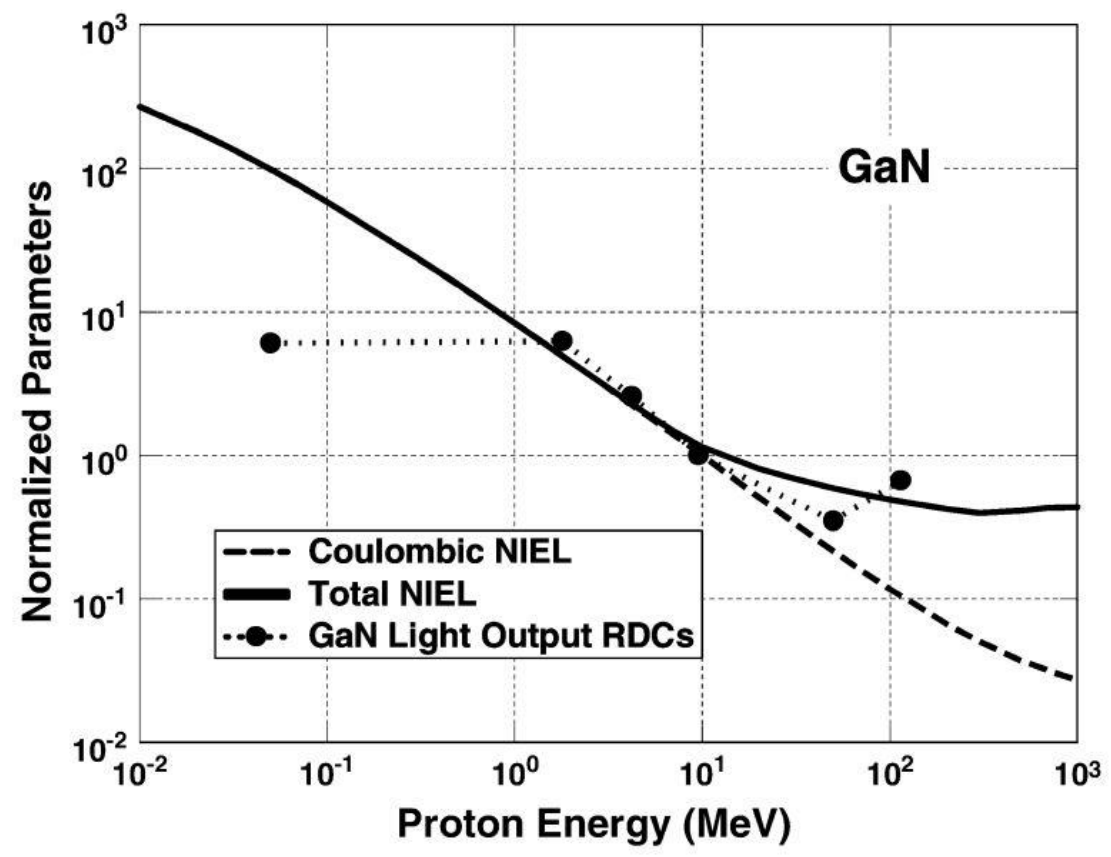

Figure 1. 13: Normalized output vs Proton Irradiation Energies [41]

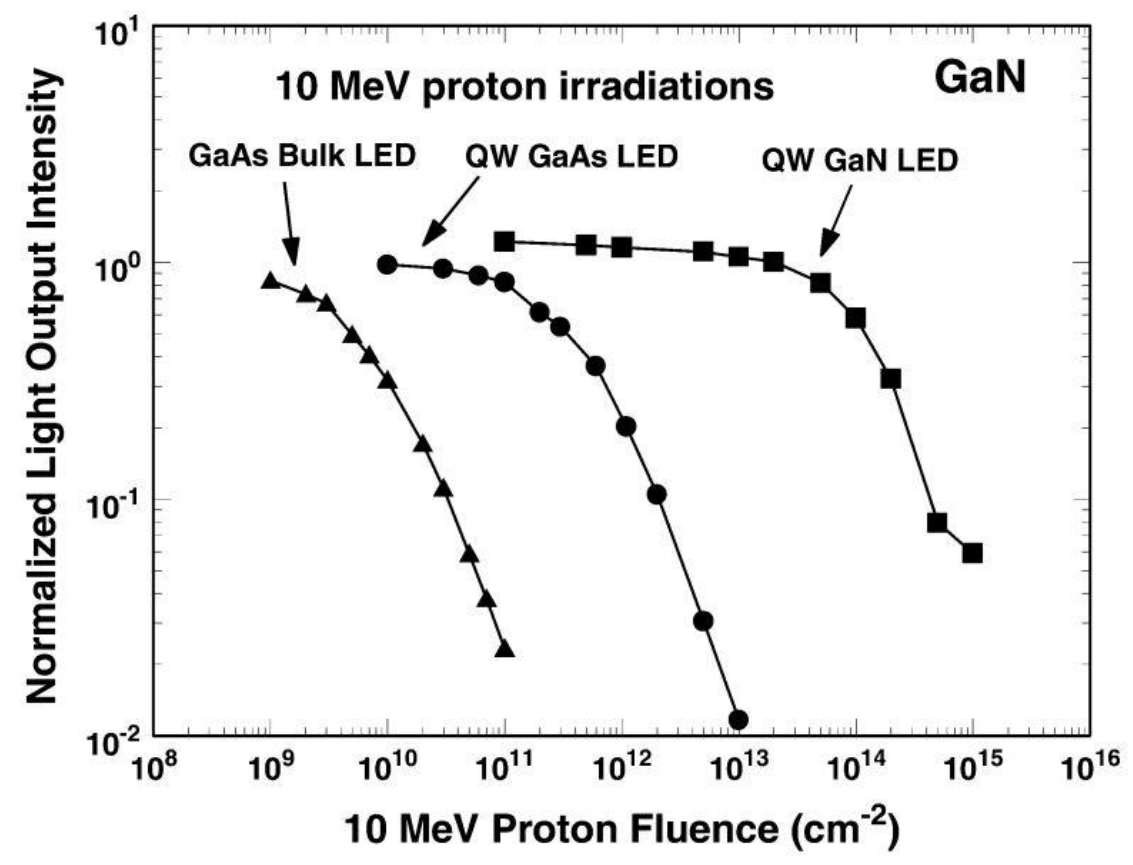

Figure 1. 14: Light Output after $10 \mathrm{MeV}$ proton irradtion of $\mathrm{GaN}$ and $\mathrm{GaAs}$ materials [41] 
From [41], it is also seen that GaN Quantum well (QW) structures degrade in optical performance two orders of magnitude after GaAs LEDs. The results presented in [42] consider the effects of proton irradiation with electroluminescent spectra and I-V characteristics, summarize in Figures 1.15 \& 1.16.

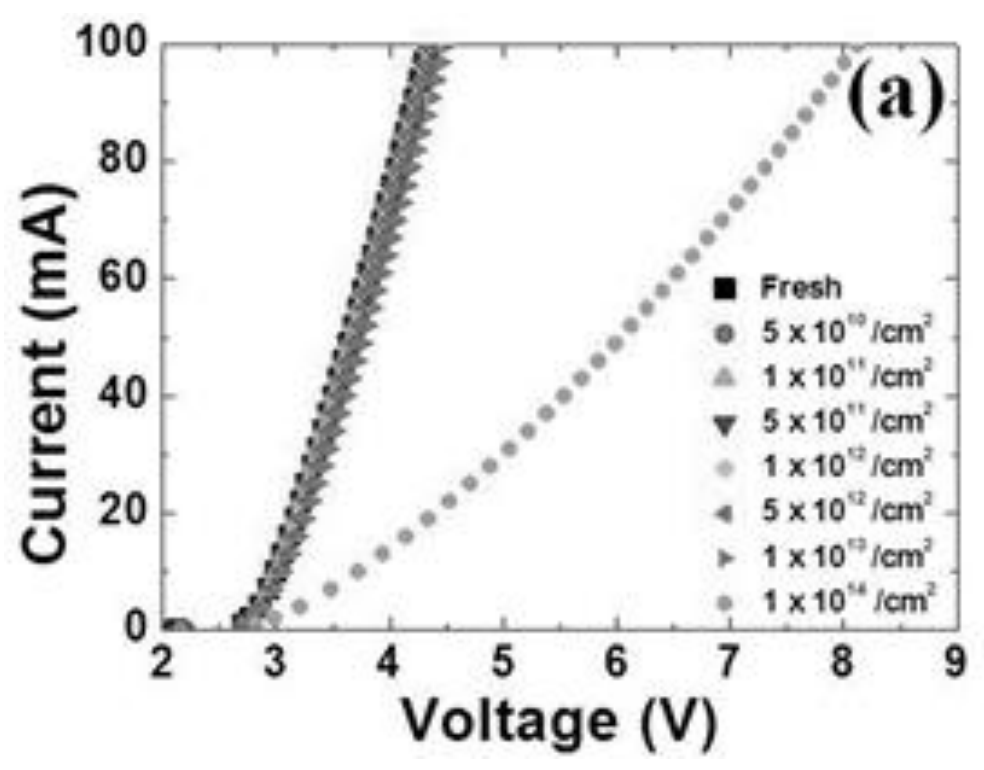

Figure 1. 15: Current-Voltage Characteristics after irradiation [42]

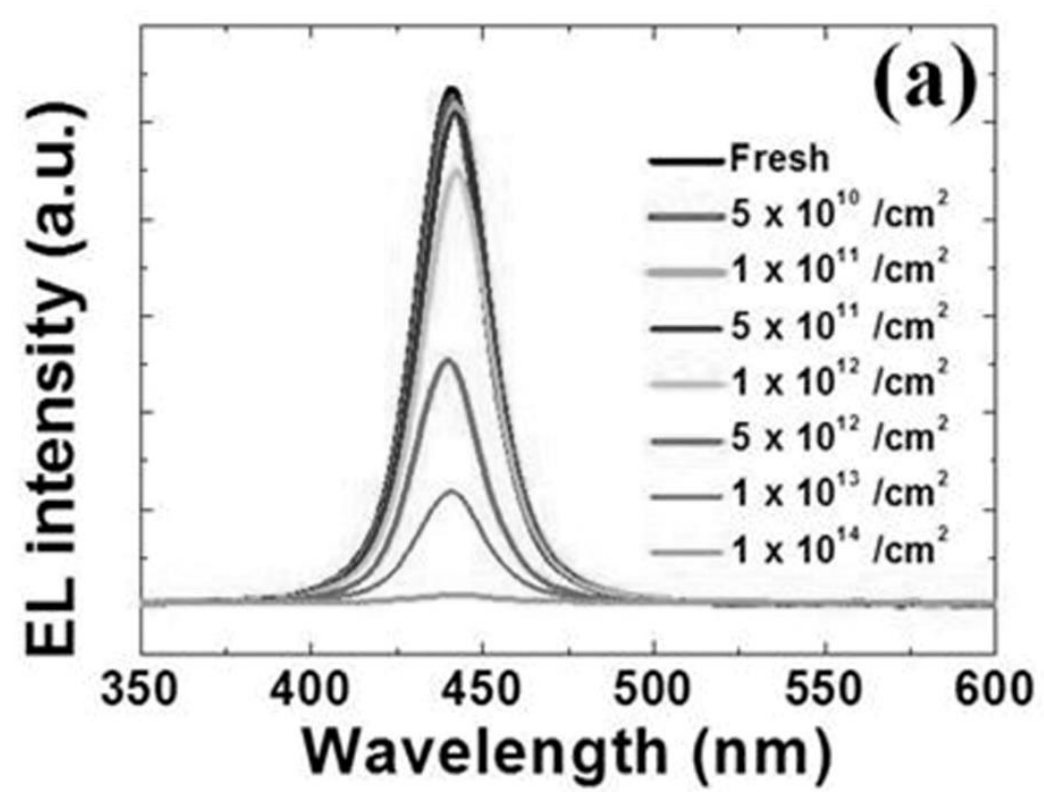

Figure 1. 16: EL output of irradiated LEDs [42] 
These studies conclude the effects of proton irradation. It was observed that the optical properties were effected before the electrical properties. While conductivity is due both to radiative recombination and non-radiative recombination, light output is related to the radiative recombination process. Because of the creation of non-radiative traps induced by damage, decreased light output is observed. It should eb noted that, while resistance, current, and light output are changing, the peak emission wavelength remains relatively close to pre-irradiated conditions.

Electron Damage - In [43], LEDs were irradiated with a range of $300 \mathrm{keV}$ to $1400 \mathrm{keV}$ electrons to examine light output intensity. It was found that the voltage threshold for lattice displacement was around $19 \mathrm{eV}$, creating noticeable damage. The effects on the LEDs showed that optical properties degraded faster than the electrical properties. Figure 1.17 illustrates the normalized light output after electron irradiation. 


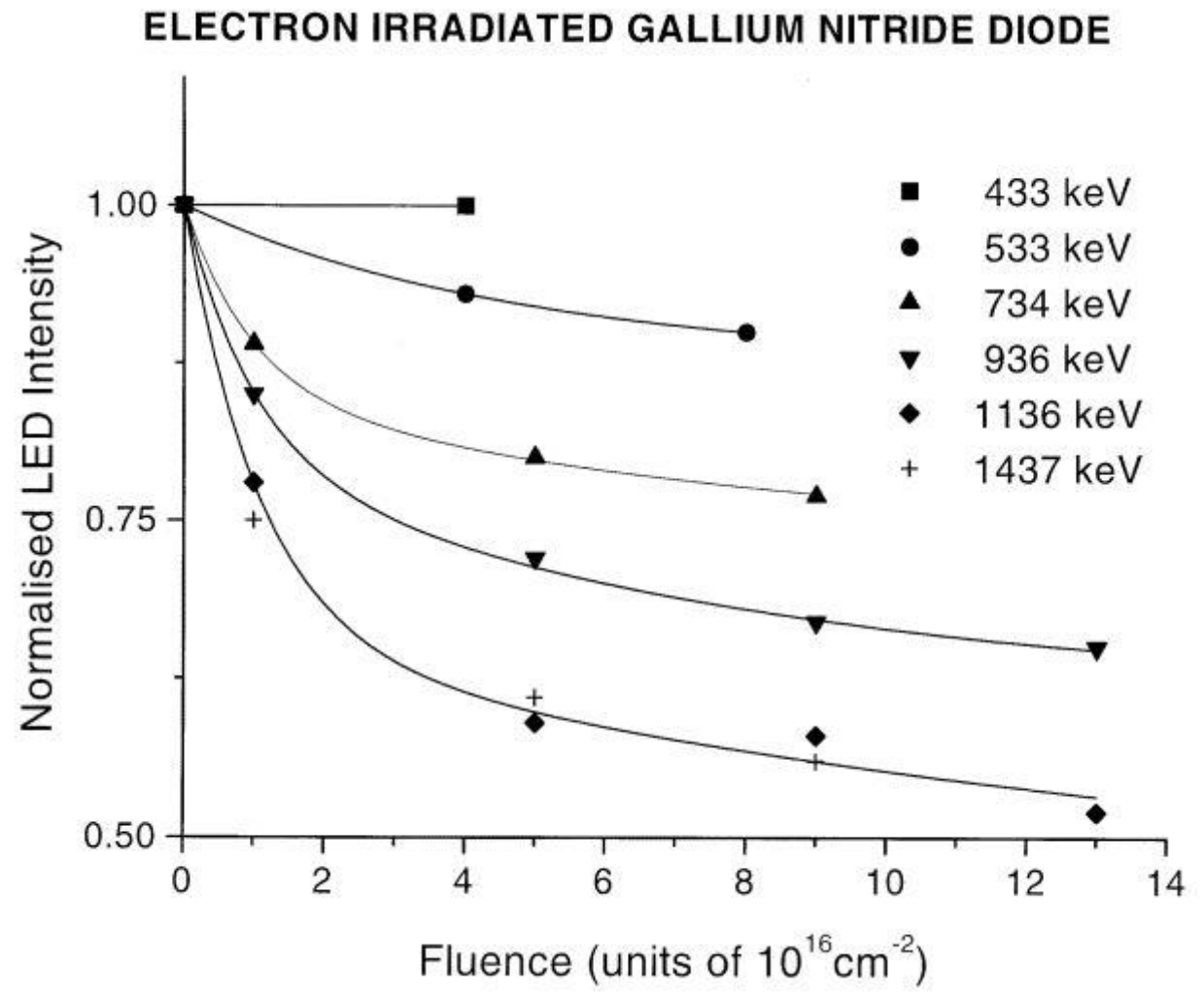

Figure 1. 17: Normalized light output vs. electron irradiation [43]

Neutron Damage - Similar effects have been seen with neutron irradiation in LEDs. Results in [44] show that neutron irradiation results in a carrier removal effect within the lattic. It was observed that an increase of saturation current occurs and was attributed to the increase in trap concentrations. Furthermore, it was observed that optical degredation was more severe than electrical effects.

Gamma ray Damage - The effert described in [46] shows that luminescity of the devices decreased as dosage increased. A .1 to $.15 \mathrm{~V}$ shift in turnon voltage was observed. Like the previous particle damages, optical output was effected the most. Because of this effect, it is also considered that these inefficiencies are due to recombination centers forming. Gamma radiation also resulted in a peak red 
shift of the wavelength emission. The peaks also showed broadening consistant with electron irradiation due to vacancies [47].

Alpha Particles Damage - GaN LEDs have been irradiated with $500 \mathrm{keV}$ alpha particles at fluences above $10^{14} \mathrm{~cm}^{-2}$ using cathodoluminescence [48]. These experiments proved to be consistant with the previously shown irradiation damage. This showed a decrease in light output and formation of deep level traps within the material.

The effects of irradiation of InGaN/GaN LEDs can be seen. The experiments show light output decrease that scaled with dosages of radiation. It is also noteworthy that the luminescent properties changed faster than electrical properties. Neutron irradiation showed a level of recorvery after annealing processes, and that electron damages do not have the largest effects on devices. Measurment of the effects of irradiation on $\mathrm{GaN}$ devices have shown that they are quite resistant to radiation, and prove to be a reliable material for electronics used for space-based applications.

\subsubsection{Temperature Effects on GaN LEDs}

Because space and the ionosphere have wide temperature ranges $(200 \mathrm{~K}-550 \mathrm{~K})$, it is necessary to examine the effects of temperature on GaN LEDs and how temperature effects I-V and EL characteristics [127]. Temperature has direct effects on the $\mathrm{p}-\mathrm{n}$ junction of LEDs and can produce change in resistance, forward voltage, current, and peak wavelength emission [128 - 130]. In [131], a method developed to determine junction temperature based on monitoring resistive change of GaN LEDs as a function of temperature is described. The effects of temperature on forward voltage can also be seen from [131]. A decrease in forward voltage was observed while temperatures was increased. 

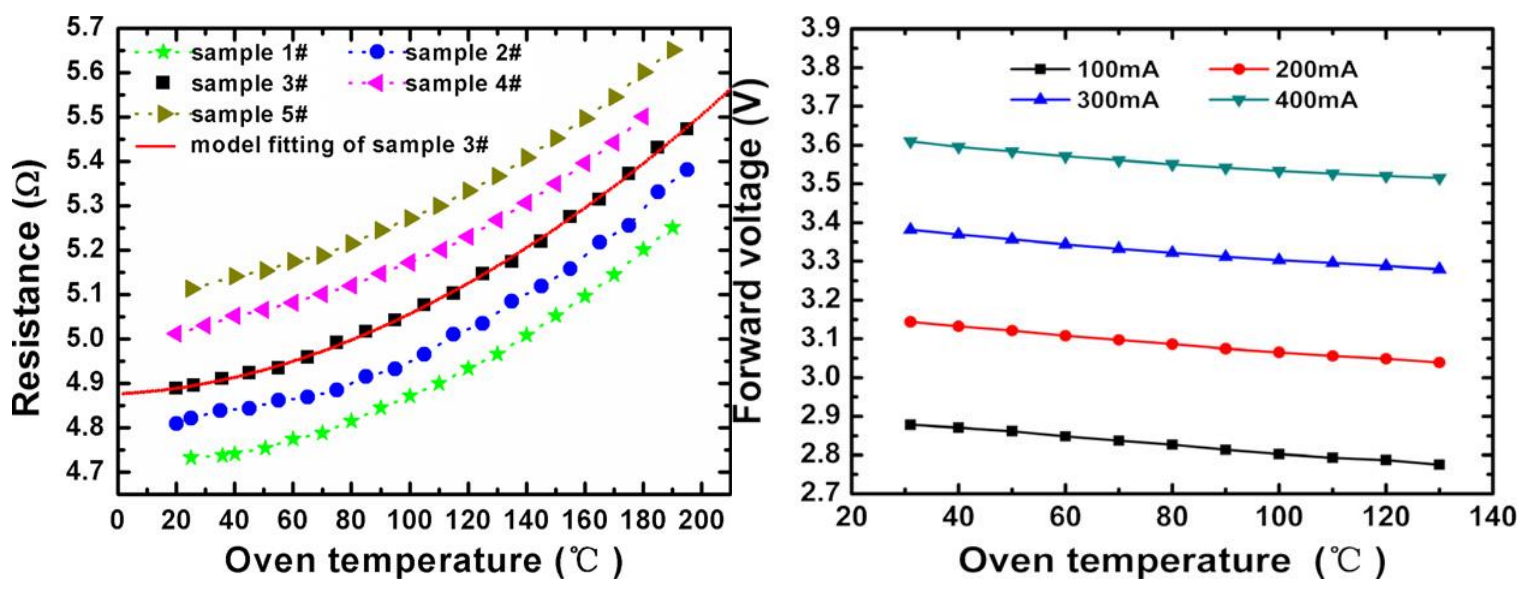

Figure 1. 18: (LEFT) Resistive change versus temperature, (RIGHT) Forward Voltage Change versus temperature [131]

Zhao has similarly reported the change in current and junction temperature at different ambient temperatures. This has shown increases in both current and junction temperature. These results show that maintaining temperature plays a crucial role in determining the electrical characteristics of LED, especially in space. Figure 1.19 shows the change in current and junction temperature at different temperatures.

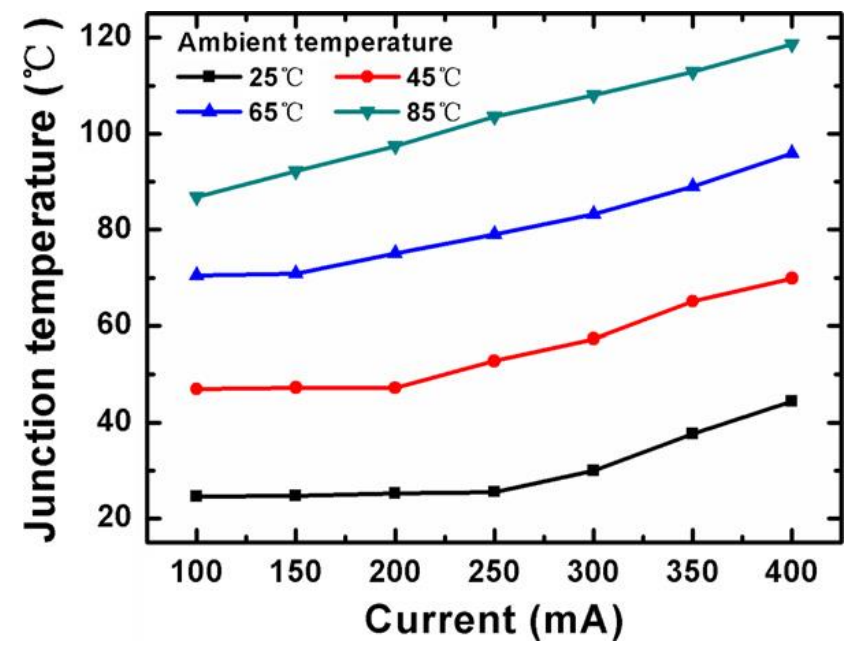

Figure 1. 19: Junction temperature as a function of current at different temperatures [131] 
Additionally, the electroluminescent spectra are influenced by temperature. This can lead to red or blue shifts in peak wavelength emission. For example, redshift may occur in InGaN LEDs as band gap energy is reduced with increasing temperature [132]. In [133], He shows that emissions peak shift occurs in high power blue LEDs as a function of junction temperature. Figure 1.20 shows the change in peak emission.

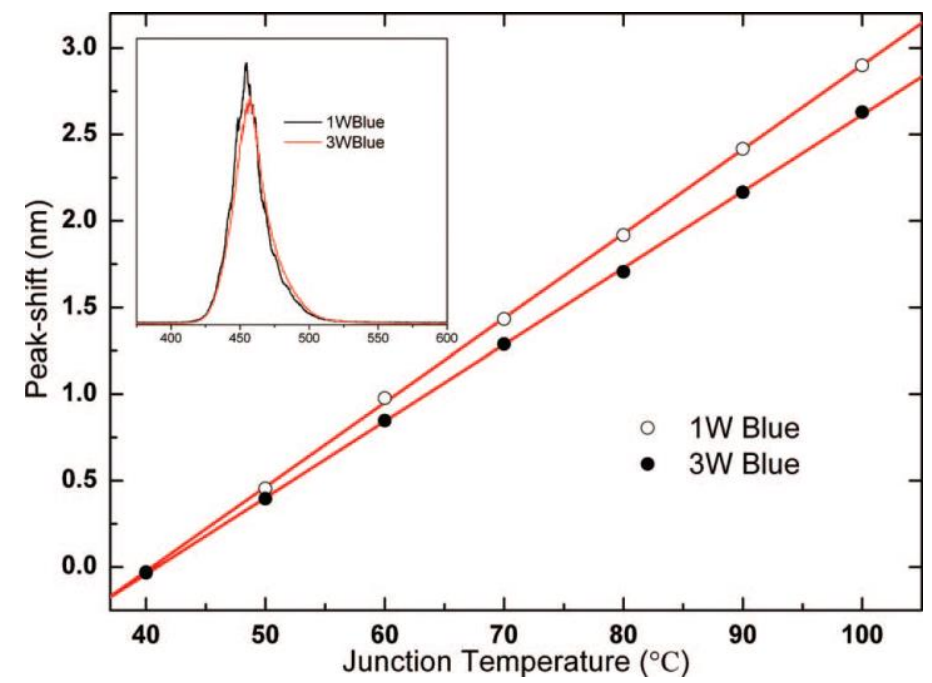

Figure 1. 20: Emission shift in peak wavelength as a function of junction temperature [133]

From the studies presented, temperature swings have a direct impact on the performance of GaN based LEDs. These effects should be taken into consideration when examining the results of the LOCC system while in space. This is because the observed changes in electrical and optical properties may not be due to radiation, but variation in junction temperature based on the relative position of the CubeSat with respect to the sun. It is also important to note that STF-1 is not stationary while on orbit and experiences rotation. This leaves different side of the CubeSat exposed to the sun at any time. For future design, thermal control of the LED characterization process should be prioritized when developing a system for characterizing LEDs while in space. 


\subsubsection{Annealing Effecting on Radiation Damage}

It has been shown that displacement damages can be removed by thermal annealing at temperatures of $800{ }^{\circ} \mathrm{C}$ [134]. Defects introduced in $\mathrm{GaN}$ by $2 \mathrm{MeV}$ protons and $0.2-2.4 \mathrm{MeV}$ electrons were removed after being annealed at around $250{ }^{\circ} \mathrm{C}$ [135] [136]. When proton, electron, and neutrons doses are increased, the density of defects is increased, the thermal stability of radiation damage is greater [135]. In GaN irradiated with high doses of protons, the luminescence intensity was not restored after annealing at $800{ }^{\circ} \mathrm{C}$. For $\mathrm{AlGaN} / \mathrm{GaN}$ irradiated with $40 \mathrm{MeV}$ protons, doses that are equivalent to decades in low-earth orbit, annealing at $300{ }^{\circ} \mathrm{C}$ restored are over $60 \%$ of initial forward current. In AlN/GaN irradiated with $5 \mathrm{MeV}$ protons showed less degradation in drain current because of the more radiation-hard heterostructure compared to AlGaN/GaN. Annealing of these devices at $250-350{ }^{\circ} \mathrm{C}$ from 5 minutes to 3 hours showed that the forward and reverse current decreased 10\%, and moved towards pre-irradiation values. [137] [138].

From the studies presented, annealing directly effects the performance of GaN materials and LEDs. These effects may not observable while on orbit, but should be taken into consideration when examining the lifetime and degradation effects of LEDs while in space. This section summarizes the effects of radiation and temperature on GaN materials and LEDs. It can be seen why GaN is chosen for applications that require stability against harsh environments.

\subsection{Trends Toward Small Satellites}

This section provides an overview of small satellites and the CubeSat subcategory, as well as trends towards their use. It also includes is an overview of their structures, standards, and methods of launching. NASA IV\&V's Simulation-to-Flight is detailed herein. 


\subsubsection{Overview}

During the post-World War II era America and the Soviet Union were matched in a "Cold War." In the years of 1957-1958, these two powers brought the dawn of the Space Age [104]. The Space Age defines the time of the Space Race, space exploration, and space technology that is seen today. This new frontier was ushered in by the launch of the world's first artificial satellite, Sputnik I. Following the launch of Sputnik I, America launched the Explorer I, followed by the U.S. Navy's Vanguard I in the same year. It has been said that congress passing the National Aeronautics and Space Act and the creation of NASA were both direct results of the launches of Sputnik I and its predecessors [105]. The launching of these artificial satellites has been hailed as great achievements of their time, and we are now seeing the fruit of these earlier advances in the emerging commercial space industry; NewSpace [106].

With the advancement of electronics and shrinking of component footprint, a resulting increase in performance and smaller systems have emerged. This allows larger satellites to be miniaturized. The Hubble Space Telescope has a mass of 11,110 kg (24,490 lb.) with its successor, James Webb Space Telescope, coming in at 6,500 kg (14,300 lb.) [107] [108]. Because weight plays a significant part in the cost of placing things into space, the miniaturization of satellites allows for a cost-effective method when launching science missions into space. Coupling cost effective electronics, compact systems, makers' communities, and the emerging NewSpace industry, shorter development times and ease of access to launch opportunities have become available [109].

Because of the miniaturization of satellites, small satellites, or "SmallSats", have been created. Small satellites come in various forms and are categorized by mass. Table 1.2 describes the subcategories of SmallSats. Please note that CubeSats fall within the category of nanosatellites and will be later detailed. These masses are referred to as gross mass, as it may include propellants. 
Table 1. 2: Classification of small satellites by mass

\begin{tabular}{|l|l|}
\hline Class & Mass \\
\hline Femtosatellites & $10-100 \mathrm{~g}$ \\
\hline Picosatellites & $.1-1 \mathrm{~kg}$ \\
\hline Nanaosatellite & $1-10 \mathrm{~kg}$ \\
\hline Mircosatellites & $10-100 \mathrm{~kg}$ \\
\hline
\end{tabular}

Examples of satellites within all categories exist. The Orbiting Picosatellite Automatic Launcher (OPAL) was designed by Space Systems Development Laboratory (SSDL) at Stanford University, Stanford, CA and was used as a mothership for six picosatellites that included StenSat, PICOSAT1.0, and Artemis [110]. OPAL demonstrated the feasibility of these spacecrafts and the ability to perform various science missions in a compact system. An example of a femtosatellite comes from Cornell University's Sprite concept, which is demonstrated as project KickSat [111]. KickSat is a 3.2 x $3.2 \mathrm{~cm}$ femtosatellites, and was a crowd funded project to demonstrate 128 Sprites inside a CubeSat [112]. The NASA developed Dellingr 6U CubeSat provides an example of nano satellite classifications. The Dellingr 6U CubeSat was designed to combat the restraints of the $3 \mathrm{U}$ CubeSat and implement lowcost, commercial off-the-shelf components (COTS). Dellingr replaced 3U CubeSats as a standard for the academic, government, and industry institutions [113].

SpaceWorks has been monitoring the global Nano and Microsatellite markets. They annually publish their findings and forecasts for free to small satellite communities. Their reviews have shown an average growth of $39 \%$ per year with a forecasted 13\% growth per year over the next six years (2016-2022), with 2,300-3,000 nano/microsatellites requiring launch [114]. Figure 1.21 describes the history of launches and projected launches over the next 6 years. 


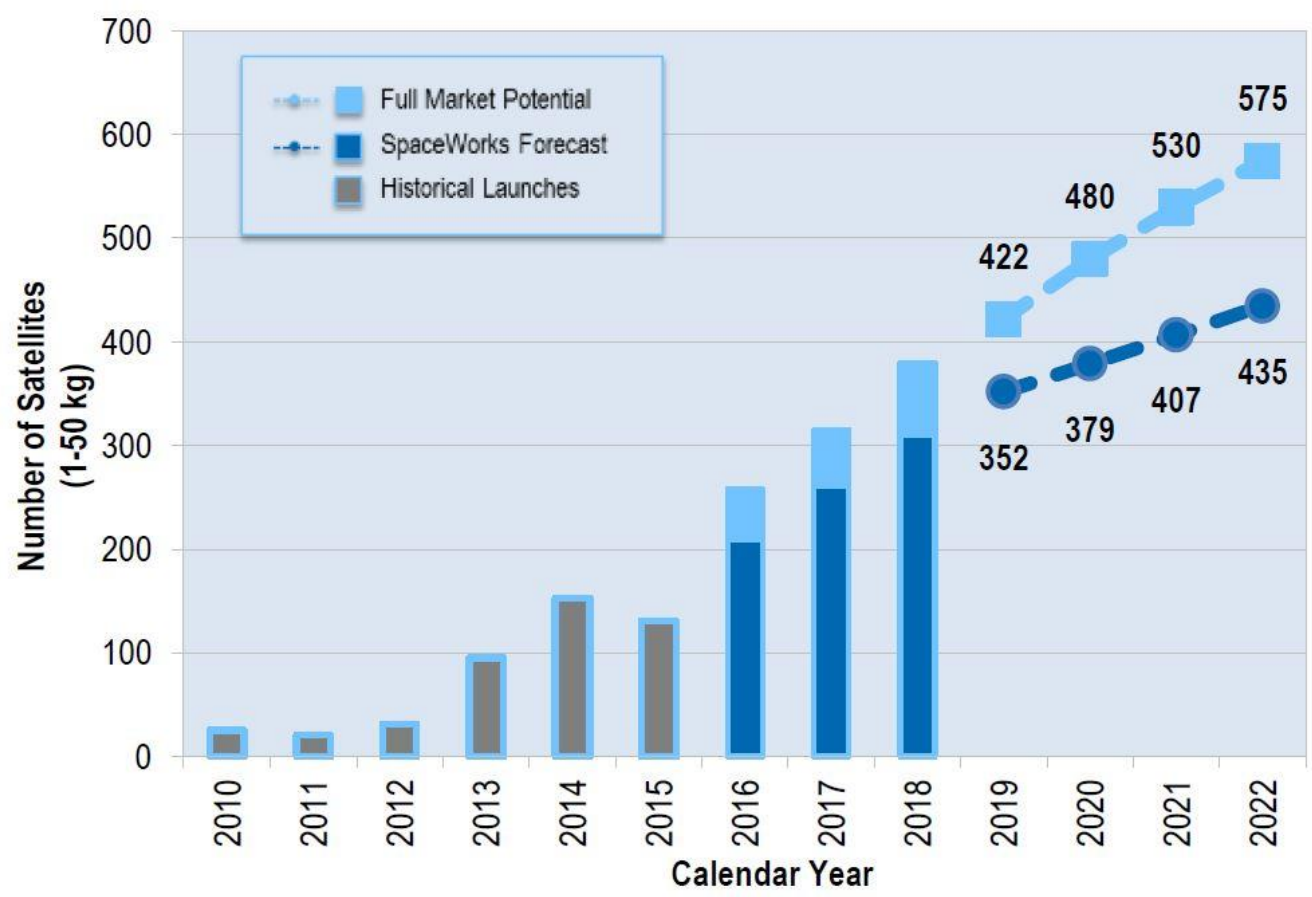

Figure 1. 21: Nano/Microsatellite Projection and History. SpaceWorks 2016 [114]

Clyde Space is one of the most prominent companies in the small satellite market. The CEO of Clyde Space has stated [115]:

I don't see it [CubeSats] slowing down. The challenge for a company like Clyde Space and others who operate in this area is whether companies can scale up fast enough to meet the demand and still maintain the quality and performance within the timeframes required by the customers.

Private investors are a driving factor in the small satellite market, and participation from multiple institutions is needed to continue upward trends [116]. 


\subsubsection{On CubeSats}

CubeSats are an increasingly popular classification of small satellites. The CubeSat standard was developed by California Polytechnic State University as a collaboration between Professor Jordi Puig-Sauri, San Luis Obispo, and Professor Bob Twiggs at Stanford University's Space Systems Development Laboratory (SSDL) [7]. The initial standardization was an attempt to lower development time and costs. Outlined in the CubeSat standards are the required parameters of dimension, mass, deployment systems, materials, etc.

Within the CubeSat guidelines, a 1U (1 unit) CubeSat shall measure as cubic with $10 \mathrm{~cm} \times 10$ $\mathrm{cm}$ x $10 \mathrm{~cm}$ dimensions. Multiple types of CubeSats exist, such as the $2 \mathrm{U}, 3 \mathrm{U}$, and up to $12 \mathrm{U}$. As an example, the $3 \mathrm{U}$ measures $10 \mathrm{~cm}$ x $10 \mathrm{~cm}$ x $30 \mathrm{~cm}$. Figure 1.22 illustrates the different configurations of CubeSats. Each variation of CubeSat has its own restrictions. For instance, each 1U CubeSat shall not exceed $1.33 \mathrm{~kg}$ of mass, or a $3 \mathrm{U}$ CubeSat shall not exceed $4.0 \mathrm{~kg}$ of mass [7]. It is important to note that, within following these guidelines of development, the power generated will be limited to the available solar-cell surface area that is available for each dimension. 3U CubeSat power profiles have shown an orbit average power of $4.9 \mathrm{~W}$ and a peak power of 9W while on the Earth's poles [119].

It has been estimated that an average cost for the full lifecycle of a CubeSat is around $\$ 52,000$ [123]. That can vary depending on the size of the CubeSat. Broadly stating, a CubeSat mission can range from $\$ 10,000$ for a $1 \mathrm{U}$ CubeSat mission to $\$ 100,000$ for a $3 \mathrm{U}$ CubeSat mission. These costs may swing based on weights and launch vehicles. To lower the expense of CubeSats, NASA created the CubeSat Launch Initiative (CLSI) which collaborates with Educational Launch of NanoSatellites (ELaNA), the ISS, and ride-share launches. This allows for launches as secondary payloads on already existing missions to be widely available to academic institutes [124]. 

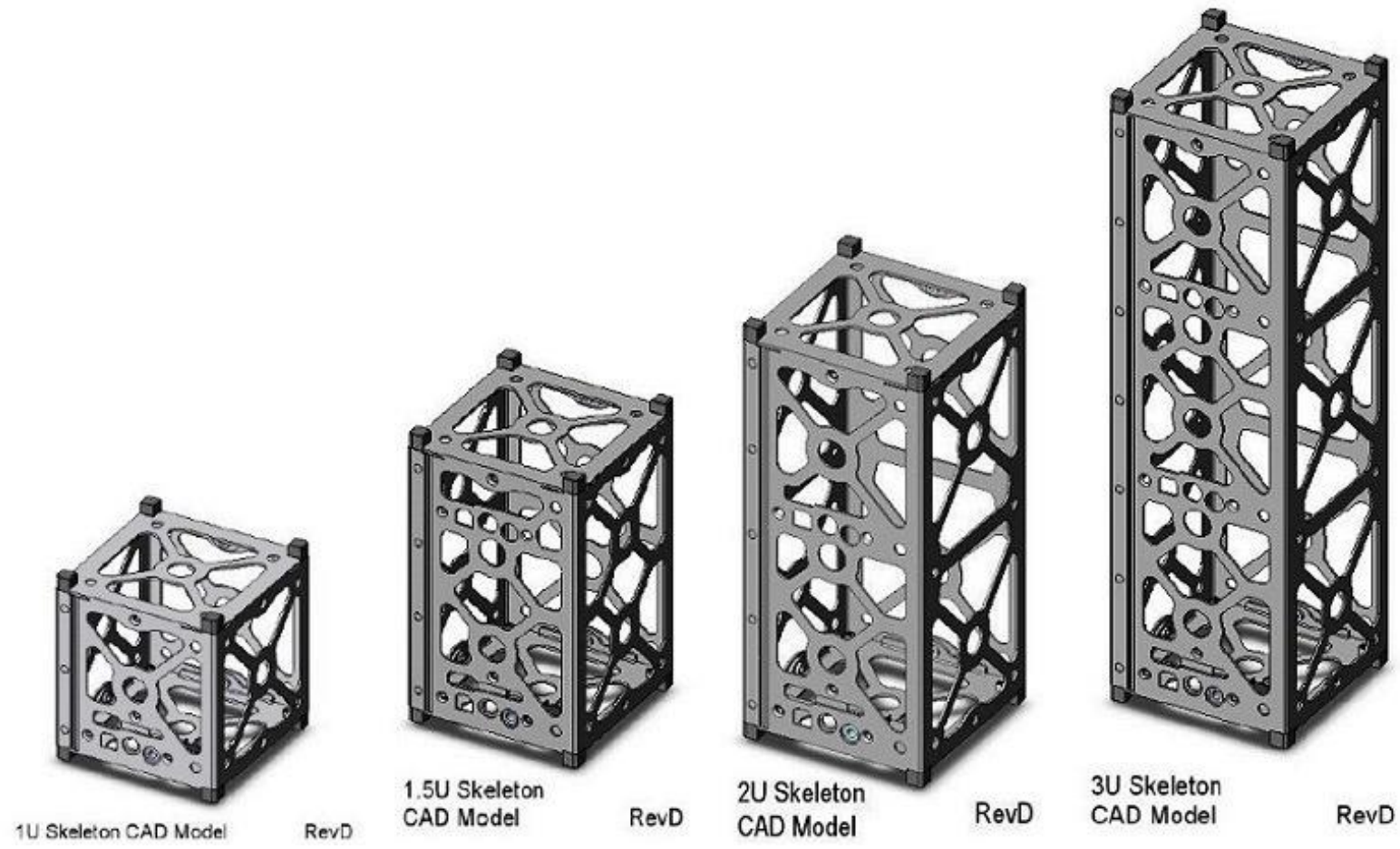

Figure 1. 22: Various CubeSat Configurations [120]

CubeSats should adhere to the standards of the Poly Picosatellite Orbital Deploying (P-POD). This is Cal Poly's standard CubeSat deployment method and system. P-PODs are attached to a rocket, the International Space Station (ISS), or other launch vehicles and deploy CubeSats into orbit [117] [118]. P-PODs work by receiving a signal and using a spring and board mechanism to launch CubeSats into orbit. Figure 1.23shows the basic 3U P-POD deployer. Popular choices for CubeSat deployment are NanoRacks CubeSat Deployer (NRCSD) (See Figure 1.23for illustration). NRCSD will be the system used to deploy STF-1. 

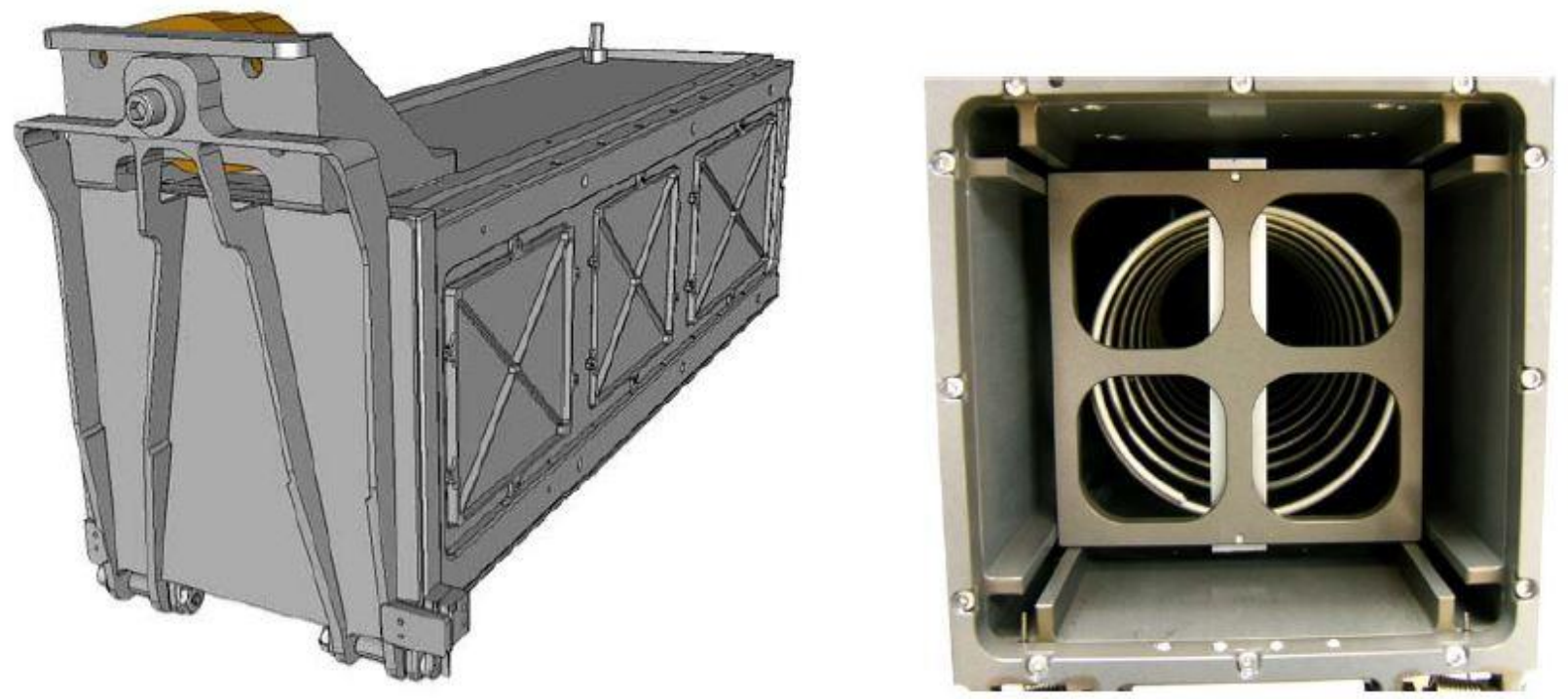

Figure 1. 23: P-POD Deployment System and Cross-Sectional Area [7]

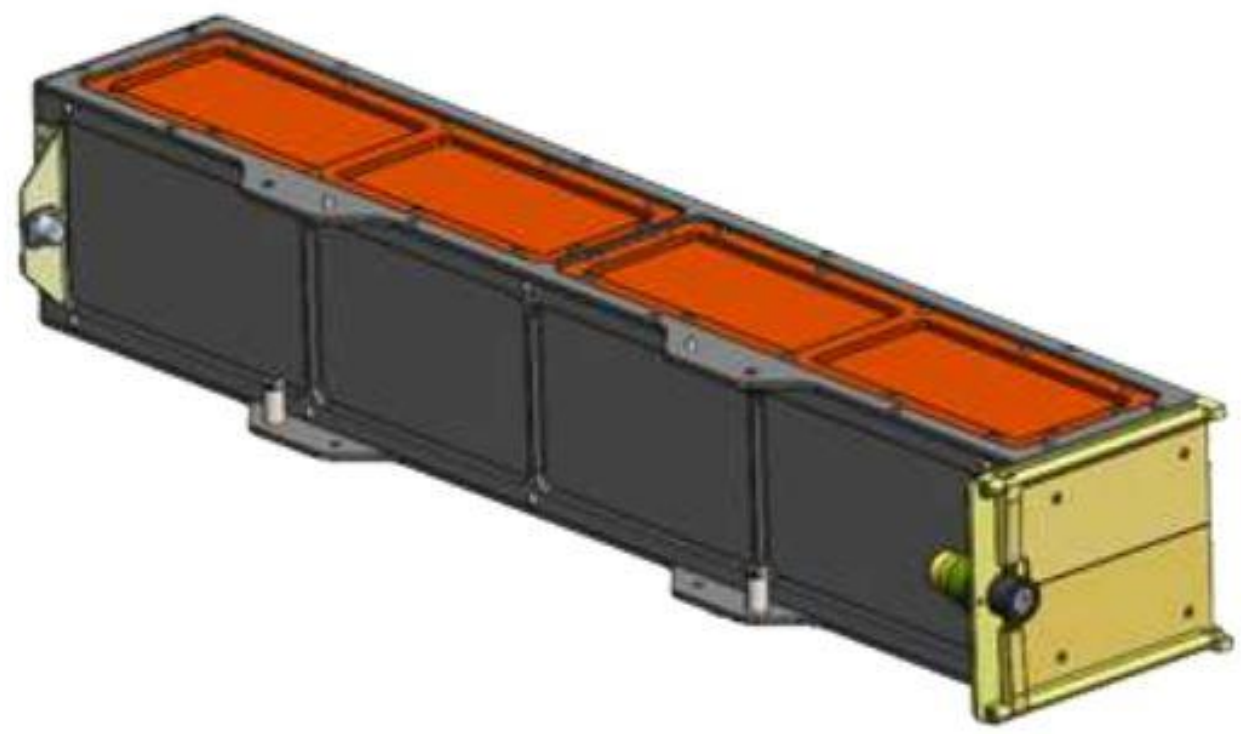

Figure 1. 24: NanoRack's CubeSat Deployer (NRCSD) [121] [122]

\subsubsection{Simulation-to-Flight (STF-1)}

Simulation-to-Flight (STF-1) is West Virginia's first spacecraft and was selected by NASA's 2015 CubeSat Launch Initiative. The CubeSat Launch Initiative comes from the White Houses' 
'Makers Initiative' and promotes the support and assistance of NASA and all 50 states working to launch small satellites over the next few years [125]. STF-1 is a 3U-specific CubeSat scheduled to launch in August of 2017. The development of STF-1 is being led by NASA IV\&V ITC team, and houses science and technology payloads from IV\&V as well as West Virginia University [126]. STF-1 is scheduled to be launched on Rocket Labs USA's Electron Rocket as part of the Educational Launch of NanoSatellites (ELaNA) XIX. The launch of the STF-1 mission has provided the ITC team and WVU students opportunity for progressing the understanding of the space environment, technology, and CubeSat development to foster growth in the small satellite industry. Figure 1.25 depicts STF-1 in its full stack. Figure 1.26 shows the STF-1 mission patch.
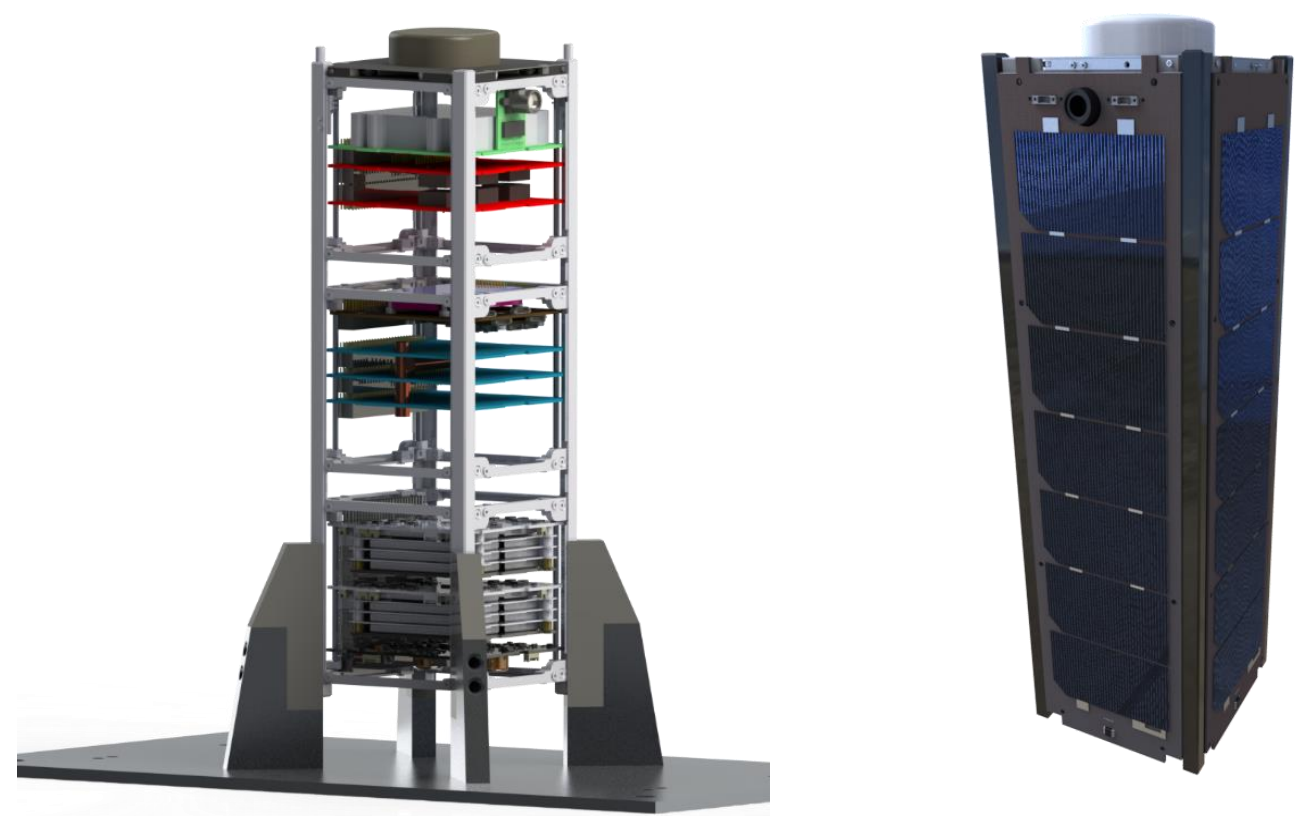

Figure 1. 25: (LEFT) 3D Rendering of Skeleton STF-1, (RIGHT) 3D Rendering of STF-1 Assembled. (Image: $N A S A I V \otimes V)$ 


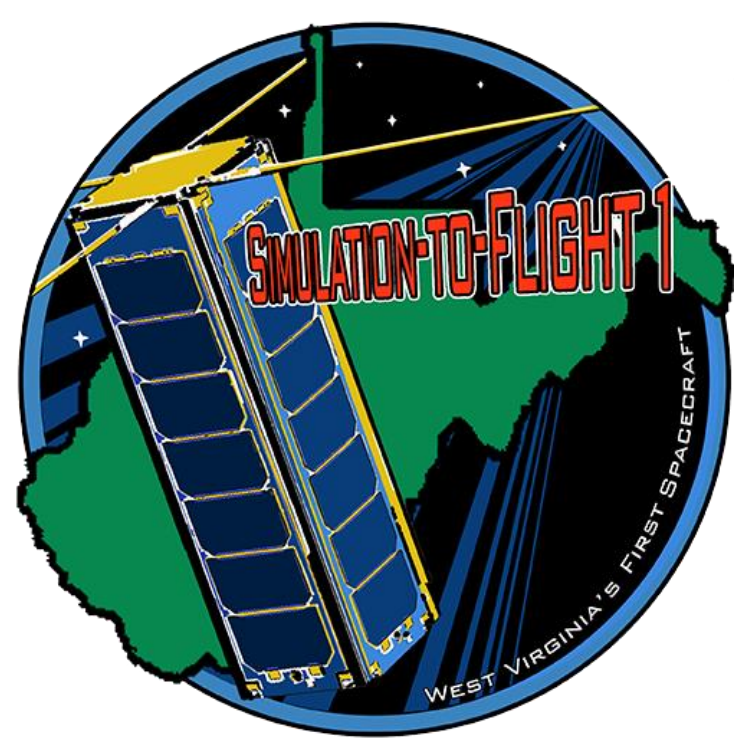

Figure 1. 26: STF-1 Mission Patch

STF-1's primary objective is focused on validation of test platforms for transitioning technologies in the development phase to flight-ready components [126]. STF-1 also houses three secondary objectives that come directly from WVU. These secondary objectives include the Mechanical and Aerospace (MAE) Department's experiment that consists of microelectromechanical systems (MEMS) and an inertial measurement unit (IMU) swarm that are part of a technology maturing program between WVU, Marquette University, and NASA Johnson Space Center. Additionally, the MAE group will be examining Precise Orbit Determination (POD) as part of a stateof-the-art Global Positioning System (GPS) to work towards developing high-accuracy GPS POD applications [126].

The next secondary science experiment comes from WVU's Physics department, which is looking to demonstrate experimental and computational methods regarding Earth's space weather. To do this, the physics department will be examining the coupling of Magnetosphere-Ionosphere and space weather in LEO. This will be achieved with an IMU for altitude information and a magnetometer, which determines magnetic field direction [126]. This allows for measurement of the 
fundamental properties of space weather and provides information for future research. This experiment works in conjunction with the LOCC system and III-V nitride materials as the resulting measurements give metrics for ionizing dosage on the LEDs.

The final secondary objective is the characterization of III-V Nitride based LEDs while under the influence of ionospheric effects. An advancement of various materials for the detection and generation of light is needed to propel the use of novel materials in space applications. This has led to the creation of an optoelectronic characterization module that contains 3 arrays of light-emitting diodes and photodiodes. The experiment will characterize devices within $380-465 \mathrm{~nm}$ wavelengths. The intent is to examine the durability of the materials used in the LEDs (GaN, InGaN etc.) and the performance of the core components in the conditions of the space atmosphere. The details of this experiment will be further examined in this thesis. Further details regarding STF-1 and its subsequent missions can be found in [126].

\subsection{Research Goals}

This thesis will provide a detailed description of the design and implementation of the LOCC system within the mission parameters of STF-1. The development of the LOCC system includes power circuit design, I2C communication isolation, power budgets, communication budgets, and PCB design and layout. Additionally, the design, fabrication, and packaging of the LEDs is described. The system was tested and assembled inside a NASA-provided cleanroom during the final assembly of the STF-1 CubeSat.

To demonstrate functionality, the output of both the Current-Voltage (I-V) characteristics and the electroluminescent (EL) characteristics of the system will be provided, along with power consumption over time. Figure 1.27 illustrates the process flow diagram for development and operation of the LOCC system for performing the characterization of LEDs onboard a CubeSat, 
along with the critical milestones of development. For continuing the LOCC system and characterizing LEDs while in space, LOCC can be used as a starting point or recreated with the given documentation to produce the platform.

Development starts with components being selected and tested for compatibility. Once the initial system has been tested in a simulation environment, PCB prototyping takes place to ensure that form and function are met. Following corrections and modification, the final PCB board can be fabricated and assembled. From here, the PCB will be integrated with the CubeSat's flight computer, and commands can be sent to LOCC for data acquisition. The CubeSat flight computer receive command codes from the ground station. These command codes send signals to LOCC to start the experiments, configure LEDs, and retrieve stored information. Once the flight computer has received LOCC's stored information, it delivers binary-encoded files that are then post-processed to decimal values and logged.
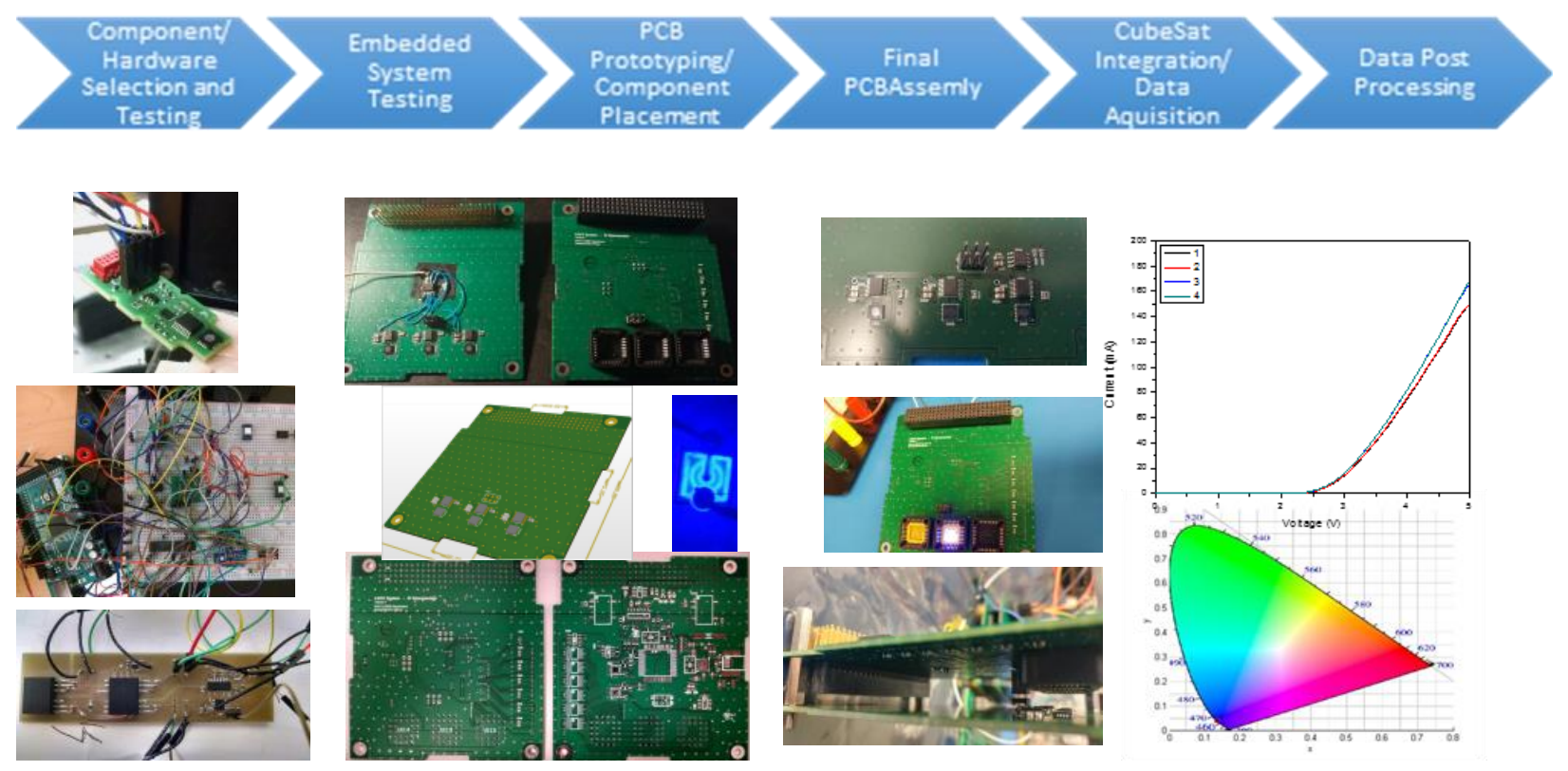

Figure 1. 27: Developmental Diagram for LOCC 
Figure 1.28 describes the overall process cycle of the LOCC system.

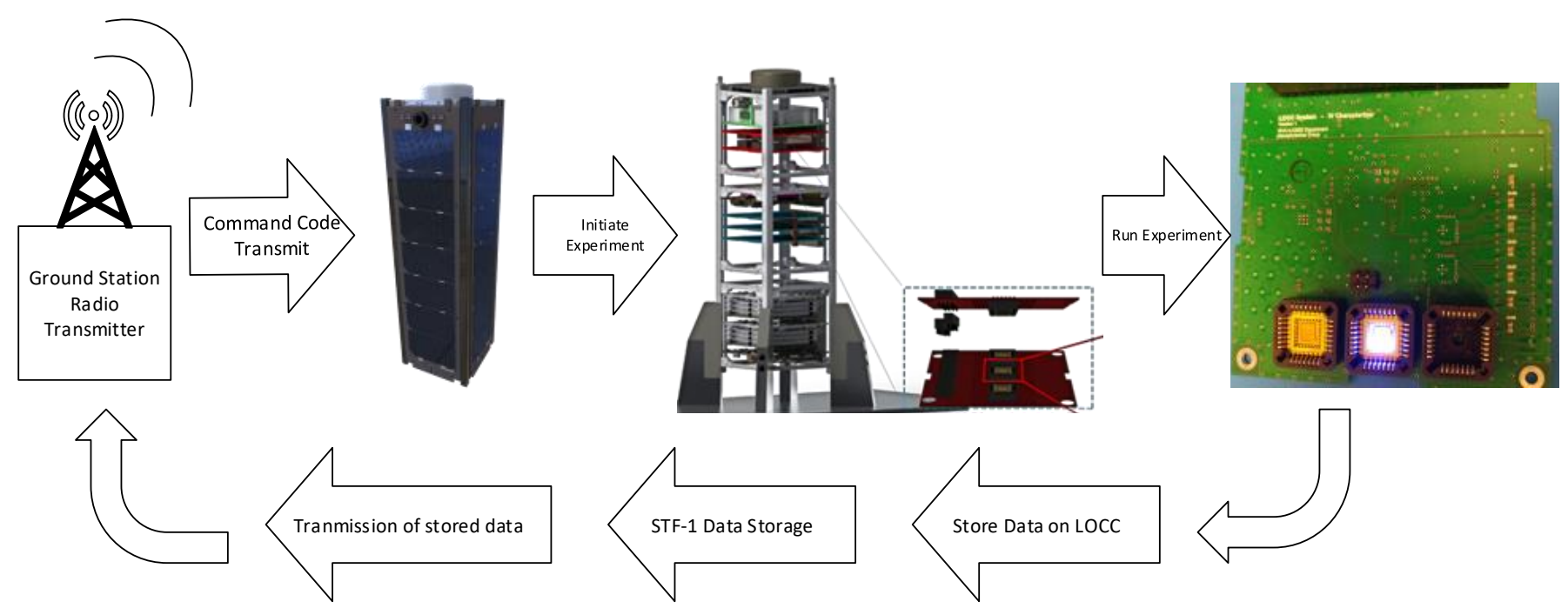

Figure 1. 28: Operational Diagram of LOCC system

The tasks involved in this research are as follows:

1: PC Board \& Circuit Development - This involves circuit design, PCB layout, embedded development, prototyping, assembly, and integration into the CubeSat. This was done using spice programs, OrCAD Capture, Altium Designer, and Arduino.

2: LED Fabrication - For the LOCC system, custom LEDs were fabricated and packaged to fit in chip carrier sockets. These LEDs are fabricated as a $16 \times 16$ array of devices with 10 major steps that include: annealing, photolithography, etching, and metal deposition. The LEDs are then packaged into chip carriers using wafer dicing, boding and encapsulation epoxies, and ultrasonic wire bonding. The LEDs fabricated were done so using commercially available substrates and fabricated with WVU SRF provided cleanroom equipment.

3: LED Characterization Via LOCC - The LOCC system has been developed to produce currentvoltage (I-V) and electroluminescent (EL) characteristics while on-board STF-1. The fabricated LEDs 
are characterized using a current sourcing method with switching to control up to 24 separate LEDs and measure the changing current. Using a grouping of analog-to-digital converters and an array of Si-PIN photodiodes the light output intensity and the peak wavelength can be determined from the operation of these components. To control these characterization methods, firmware has been developed for switching, ramping, and data acquisition. STF-1's flight computer sends command codes to the LOCC system to initiate one of two experiments. Data is stored in LOCCs onboard EEPROM. This data is then sent to STF-1 to be stored until the ground station can download the data.

The developed LOCC system and the research performed provide new tools and ideas for the way electronics and optoelectronics are thought about in the space environment. LOCC is built to fit in the CubeSat platform, adhering to power and data budget requirements while being able to deliver electrical and optical characteristics of the onboard LEDs. The fundamental operation of LOCC allows for reproduction, scalability, modification, and improvement for a variety of environments. This tool paves way for research and allows for those interested in space exploration to develop more sophisticated devices with expanding reliability, lifetime and reduced costs.

\subsection{Thesis Overview}

The work presented within looks at applying optoelectronic characterization methods into a CubeSat system to study the effects of radiation in semiconductor materials while on orbit. The optoelectronic characterizer is presented as a tool for supporting research done on improving the lifetime and reliability of optoelectronics for space-based applications. This thesis is organized as follows: Chapter 2 describes the theory of LED operations, displacement damages and ion stopping in both LEDs and PDs, and thermal effects. Chapter 3 provides a hypothesis and background on the characterization method and what is looked for from the LOCC system. This also includes the 
schematic of each of LOCC's modules, PCB layout, firmware, and software interfacing. Chapter 4 describes the LED fabrication and packaging processes. Chapter $\mathbf{5}$ provides a system evaluation to illustrate functionality with I-V and EL characterization results, light output intensity, power consumption, data budgets as well as costs. The two main experiments performed are the I-V characterization and the EL characterization. These two experiments provide the bases for all other information retrieved from this system. Lastly, Chapter $\mathbf{6}$ concludes the thesis and examines future work that can be done regarding the LOCC system and LED research for space-based applications. 


\section{Chapter 2: Theory}

\subsection{Introduction}

This chapter discusses the operation of light emitting diodes and accumulated radiation damage in III-V GaN materials. This section will look at their theoretical models as well as effect of temperature on $\mathrm{GaN}$ materials and the changes that may occur. An understanding of how radiation particles are implanted into semiconductor materials is also presented.

\subsection{LED Basics and Operation}

Light generated by an LED requires that electrons undergo recombination with their counterpart, holes. Electron-hole recombination is the basis for the emission of photon energy from semiconductor materials. The largest commercial demand for illumination is the production of visible light that is comprised of wavelengths from $400 \mathrm{~nm}$ to $700 \mathrm{~nm}$ [128].

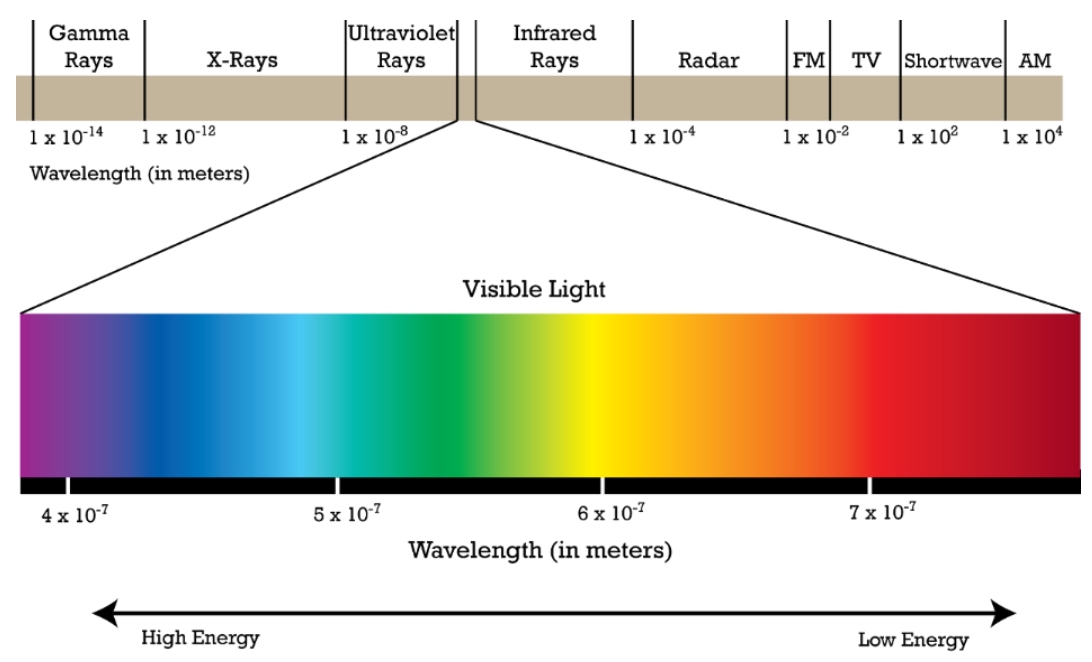

Figure 2. 1: Electromagnetic Spectrum [127] 


\subsubsection{Direct and Indirect Band Gap Semiconductors}

It is important to note the fundamental operation of light generation in semiconductor materials. Semiconductor materials are generally divided into two categories: direct bandgap and indirect bandgap materials. These bandgap structures consist of the conduction band and the valence band. The valence band consists of stationary electrons that exist around their respective atoms. Electrons in the conduction band can move from vacancy to vacancy within the atomic lattice. The momentum of electrons plays a significant role in the transition from conduction to valence band. Electrons and holes with the same momentum, as found in direct bandgap semiconductors, will transition, and electron-hole pairs will recombine to produce a photon. Oppositely, electrons and holes with different momentums may also recombine. This can be seen in indirect bandgaps. Transitioning electron-hole pairs may generate phonons, resulting in lattice vibrations or the production of heat.

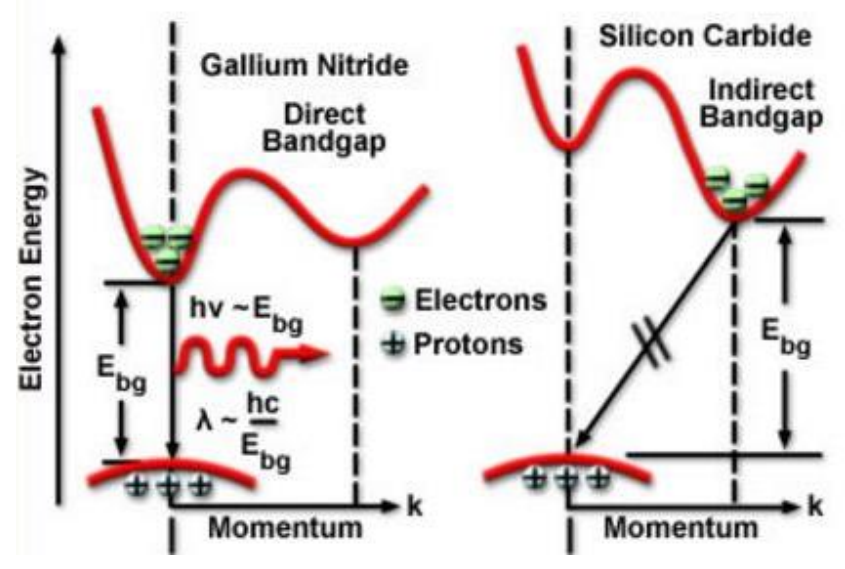

Figure 2. 2: Energy Band Diagram of Direct (Gallium Nitride) and Indirect Bandgap (Silicon Carbide) [129]

Group III-V nitrides consist of direct bandgap materials and are often comprised of binary, ternary, and quaternary atomic structures. Respectively, these can be seen as GaN, InGaN, or AlInGaN. 


\subsubsection{Materials and Structures}

The LOCC system is designed to characterize III-V nitride LEDs while on-orbit. Group III$\mathrm{V}$ nitride based materials consists of engineered energy bandgaps that fall within the $.7 \mathrm{eV}$ to $6.4 \mathrm{eV}$ ranges. These approximated bang-gap energy boundaries are formed by binary materials Indium Nitride (InN) and Aluminum Nitride (AlN). Gallium Nitride $(\mathrm{GaN}$,$) is the focus of this research,$ possesses a band gap that falls around $3.4 \mathrm{eV}$ [130]. Proper grown and engineering of III-V materials allows for the tuning of bandgaps. This is done by forming tertiary and quaternary compounds such as Indium Gallium Nitride (InGaN) or Aluminum Indium Gallium Nitride (AlInGaN). The tie-lines between the points in Figure 2.3 show that a combination of these materials can produce a wide range of emission wavelengths lying within the visible spectrum.

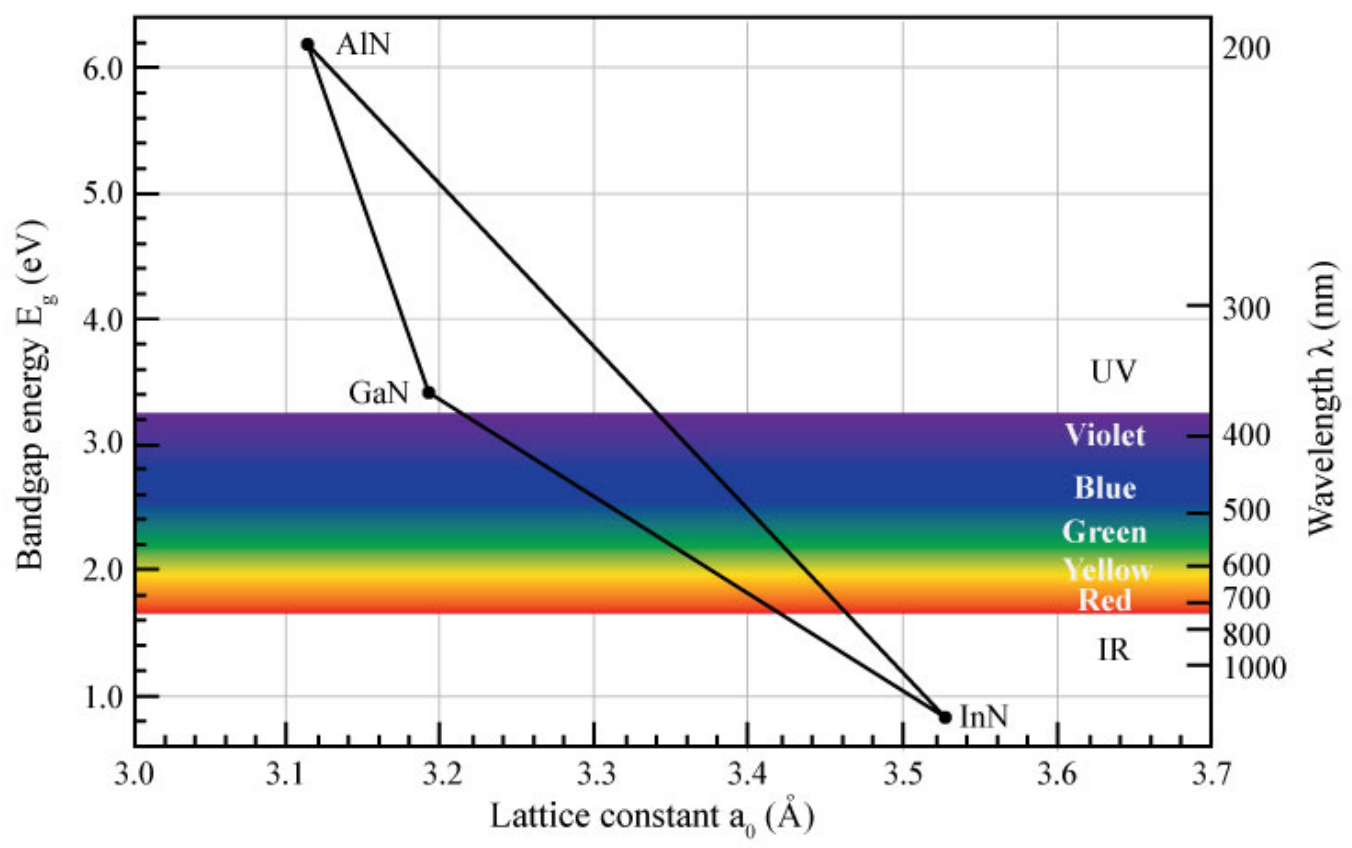

Figure 2. 3: Band Gap Energy vs. lattice constant [130] 
For creation and bandgap energy tuning of these materials, the alloy properties follow the Vegard's law:

$$
P_{A_{x} B_{1-x} N}=x P_{A N}+(1-x) P_{B N} .
$$

Here, $P_{A N}$ and $P_{B N}$ represent material properties of $\mathrm{InN}$ and $\mathrm{GaN}$, and $x$ is fractional. For calculating the resulting bandgap of the heterostructure, an empirical equation can be used.

$$
E_{I n_{x} G a_{1-x} N}^{g}=x E_{I n N}^{g}+(1-x) E_{G a N}^{g}-x(1-x) b_{I n G a N} .
$$

It follows that $E_{G a N}^{g}$ is the material band gap energy of $\mathrm{GaN}$ at $3.4 \mathrm{eV}$ and $E_{I n N}^{g}$ is the material band gap of InN at $.7 \mathrm{eV}$, and $\mathrm{b}$ is the bowing parameter. From here the operating wavelength can be determined using band gap energy (or vice versa) using

$$
E_{g}=\frac{h c}{\lambda}
$$

where $E_{g}$ is the band gap energy, $h$ is Plank's constant, $c$ is the speed of light and $\lambda$ is the wavelength.

The crystallography of group III-V based semiconductors can come in both wurtzite and zinc blende structures. Generally, III-V nitrides exist in a wurtzite crystal structure, and will be the focus of describing the atomic structure of the material used in this work [131]. Figure 2.4 shows the general structure of both wurtzite and zinc blende. 

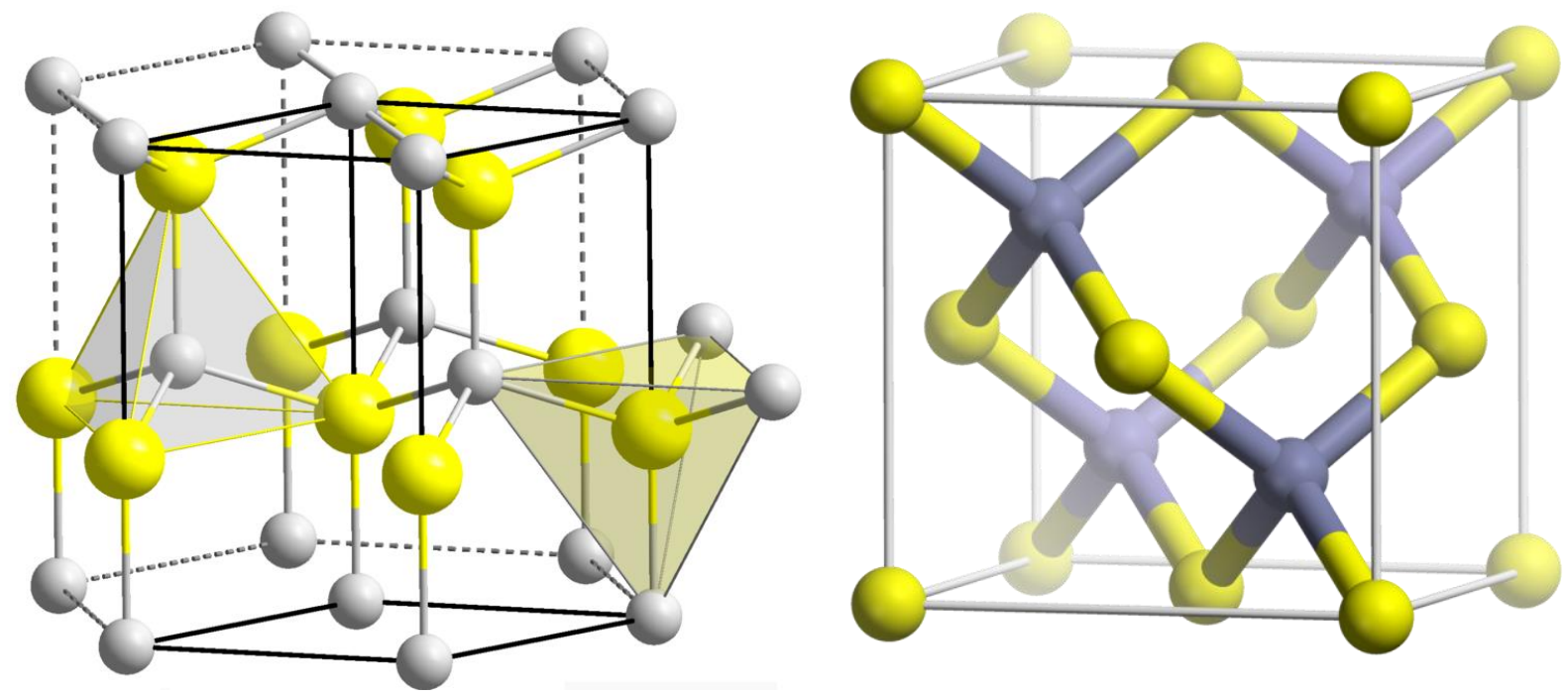

Figure 2. 4: (LEFT)Wutrite Crystal Stucture, (RIGHT) Zinc Blende Crystal Structure [133]

\subsubsection{P-N Junction Theory}

Light emitting diodes are comprised of semiconductor material that are given a positive charge (p-type), with an excess of holes, and negative charge (n-type), with an excess of electrons. These materials gain their positive and negative charge by doping the base materials using methods such as ion implantation, diffusion, or epitaxy. When these materials are placed together a region known as the depletion region is formed. The excess of positively charge holes are forced back by the positive ions that have been revealed with the pushing of electrons from the stationary negatively charges ions. The newly-formed built in electric field prevents the flow electrons until an electric potential is applied. Figure 2.5 illustrates a $\mathrm{p}$-n junction at equilibrium temperature with heavier doping of acceptors 


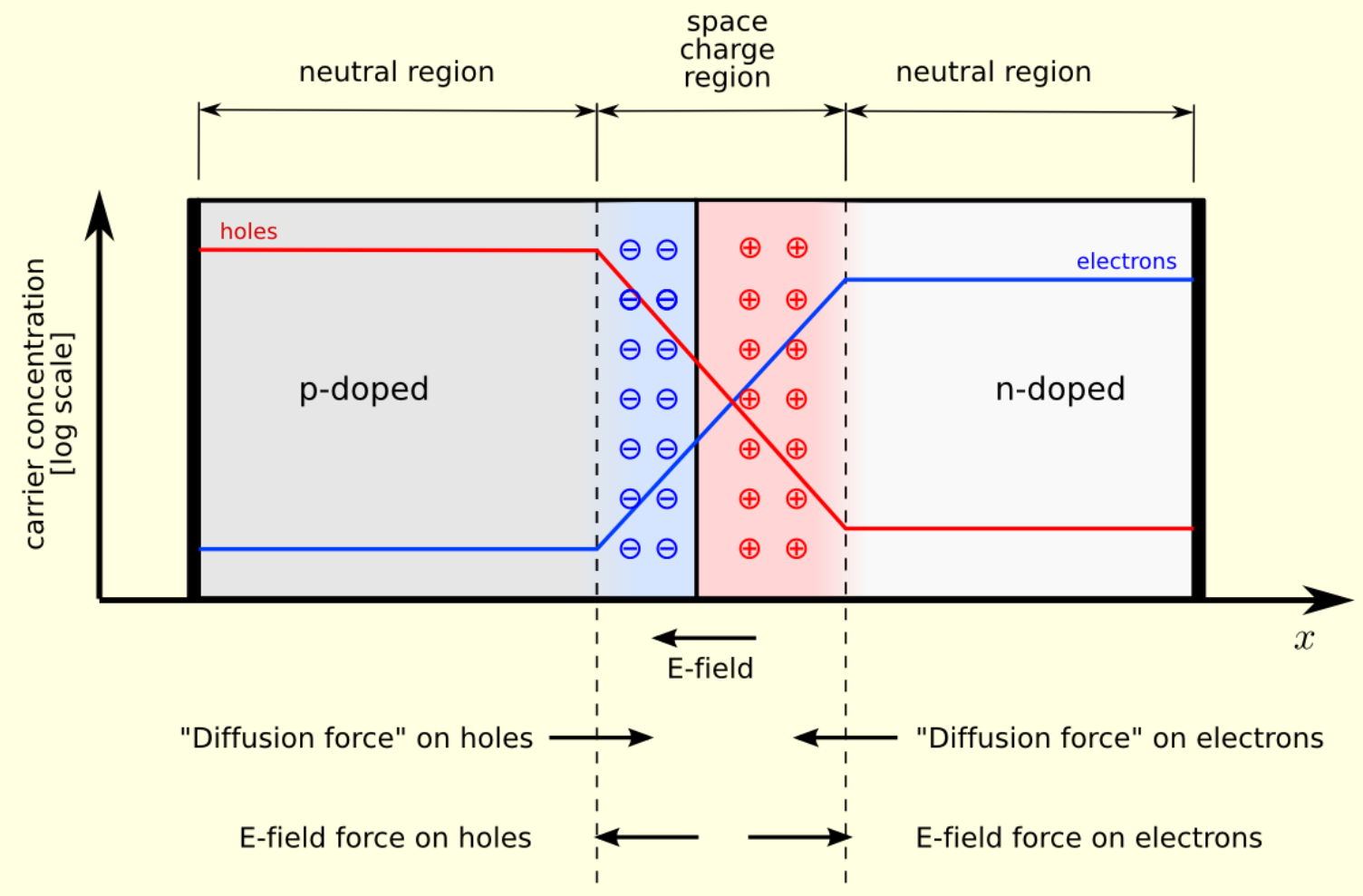

Figure 2. 5: Asymmetric p-doped P-N junction at thermal equilibrium and zero-bias voltage [134]

To operate an LED, the space charge layer that has been created by the repulsion of electrons and holes must be overcome. This is done by applying electromotive force, voltage, across the $\mathrm{p}-\mathrm{n}$ junction. Once the voltage is applied, the free electrons and holes are swept across the space charge layer, creating a current flow. With both holes and electrons flowing against each other, the process of recombination begins. The energy from the sub-particle recombination is released in the form of photons. The production of light within the material is called electroluminescence. The desired emission energy or wavelength can be calculated via the equations mentioned above. Figure 2.6 illustrates the operation of a LED. 


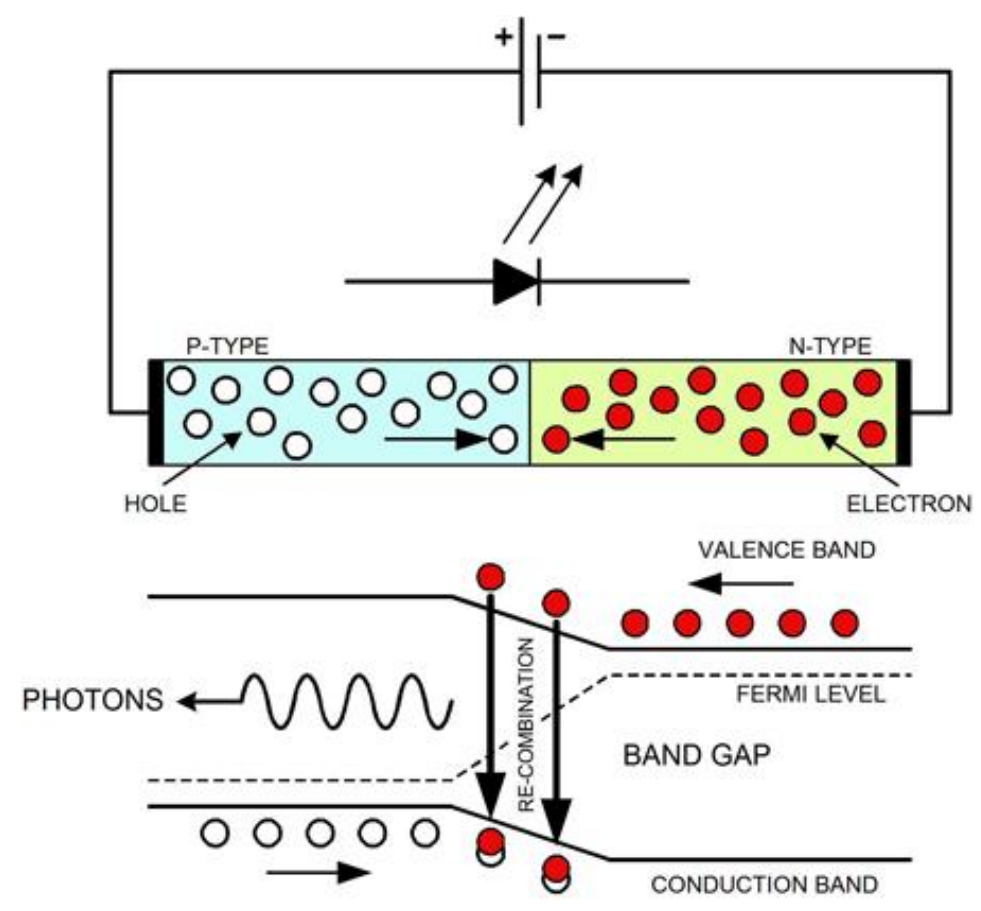

Figure 2. 6: LED Circuit and band Diagram [135]

The $\mathrm{p}-\mathrm{n}$ junction can also be connected in a reverse and a voltage applied. In this configuration, because the negative terminal is connected to the positive charge, holes move away from the n-type barrier. This is the same for electrons. Because the positive terminal of the voltage source is connected the negative charge of the $\mathrm{p}-\mathrm{n}$ junction, the electrons pull away from the barrier, leading to an increased electric field or spatial-charge layer. When photon hits the diode, electron-hole pairs are created. This process is known as the photoelectric effect. Absorption occurs in the space charge layer; these generated hole pairs are the swept from then junction and produce photocurrent. This turns the $\mathrm{p}-\mathrm{n}$ junction into a photosensitive device that produces current proportional to the light falling on the active region of the material. With forward or reverse bias configurations, the $\mathrm{p}-\mathrm{n}$ junction can potentially act as an LED by spontaneous emission or as a photodetector through absorption and generation, though their activation energies may vary based on material and structure. Figure 2.7 illustrates the basic operations of each device. 

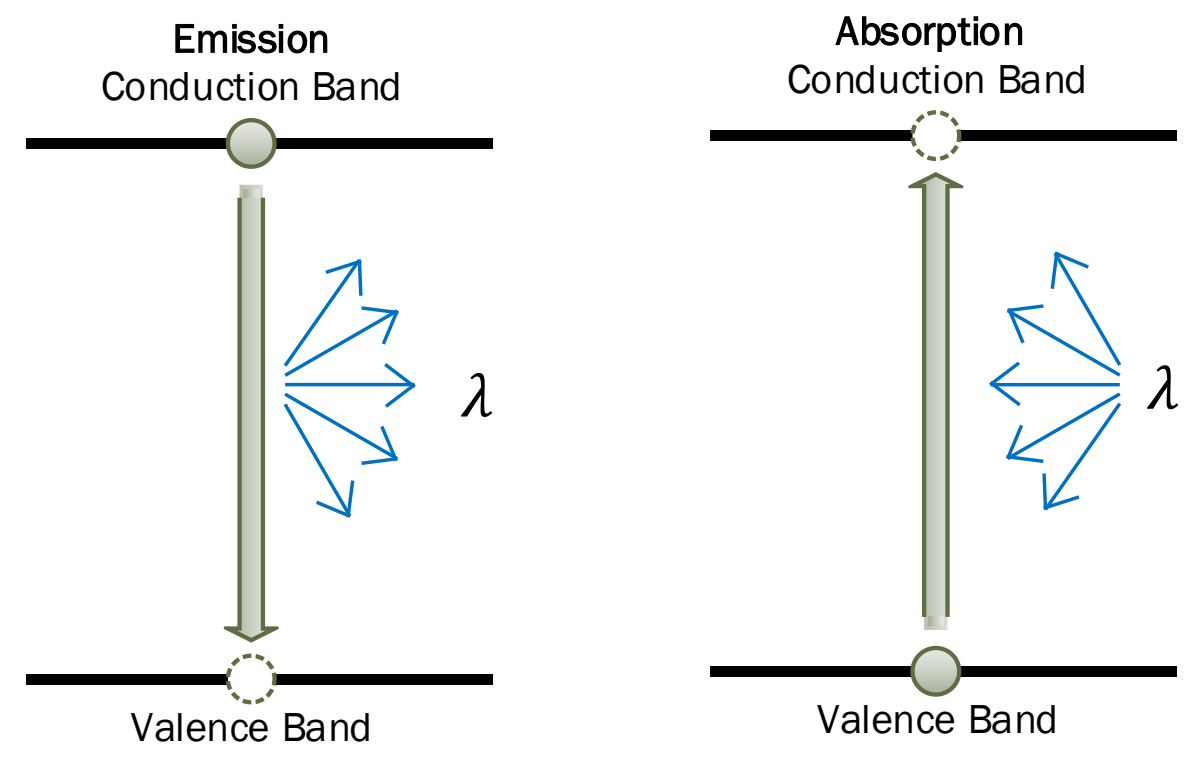

Figure 2. 7: Emissions and Absorption operations of p-n junction.

The recombination of electron-hole pairs follows a Boltzmann distribution and the emission intensity is expressed as the following:

$$
I(E) \propto \sqrt{E-E_{g}} e^{-\frac{E}{k T}}
$$

Here, $E$ is the electric potential, $E_{g}$ is the bang gap energy, $k$ is the Boltzman constant, and $T$ is temperature. Oppositely, light that is absorbed into a material is a function of distance into the material and is based on the following equation:

$$
I(x) \propto I_{o} e^{-a x}
$$

where $a$ represents the absorption coefficient of the material $x$ is the distance into the material, and $I_{O}$ is the light intensity at the material's surface. The operation described above gives the coupled 
concept of LEDs and photodetectors. Utilizing these core concepts of emission and absorption, optoelectronic devices can be tuned and configured for sunlight detection, auto-focusing, optical communications, barcode scanners, position sensors, etc. This thesis focuses on the forward characterization of LEDs. Commercial photodiodes are used in the characterization of emission spectrum.

\subsection{LED characterization}

LOCC will control voltages to characterize III-V nitride LEDS. This is needed to examine the forward voltage characteristics, current throughput, and light output during the lifetime of the device on orbit. LOCC is broken into two systems, the I-V characterization module and the EL characterization module. Below, the characterization methods employed in these modules will be described in detail.

\subsubsection{I-V Characteristics}

The LOCC system is built with a voltage ramping module because current as a function of voltage is to be examined. The current-voltage characteristics represent the amount of current traveling through the device over a range of $0 \mathrm{~V}$ to $5 \mathrm{~V}$. This allows the determination of dependent variables such as the device resistance, turn-on voltage, and allows for examination of current versus light output. Figure 2.8 illustrates measurements for an Schottky diode [145]. 


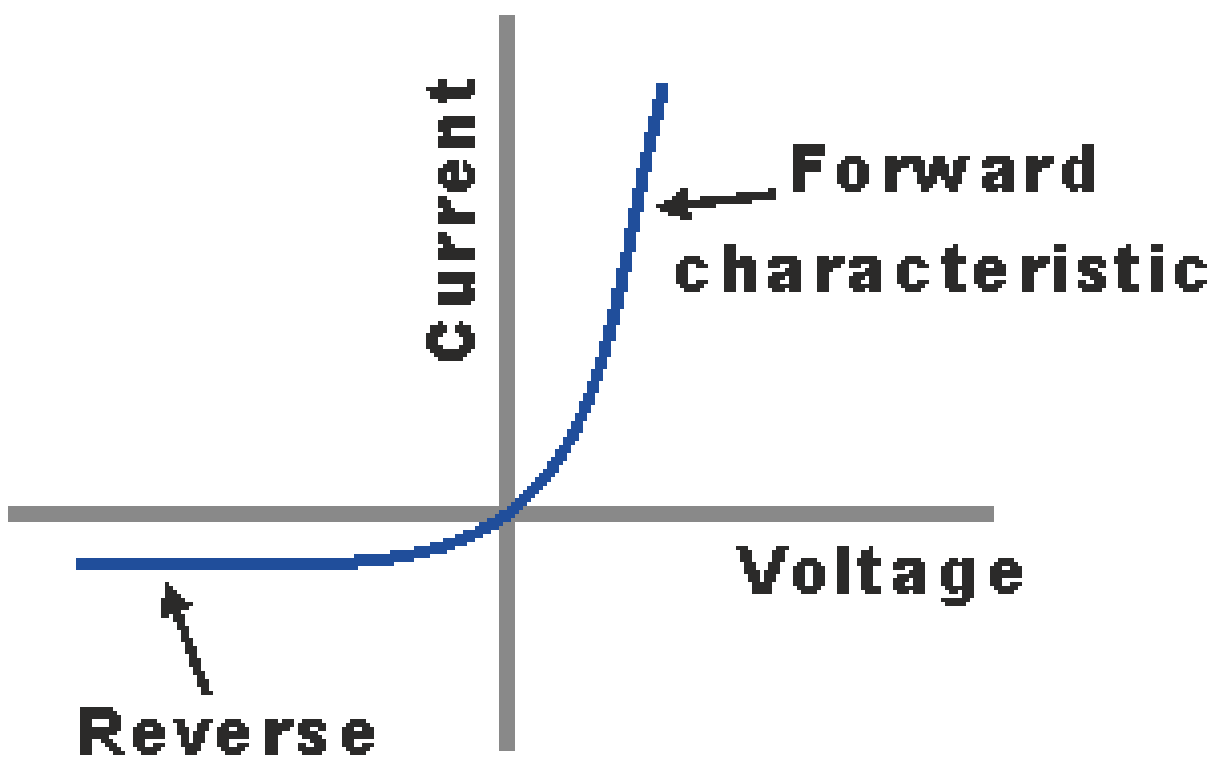

Figure 2. 8: Schottky Diode I-V characteristic curve [145]

This graph can be represented by the equation:

$$
I=I_{S} e^{\frac{V_{d}}{n V_{T}}}-1
$$

where $I$ is the current through the device, $I_{S}$ is reverse saturation current, $V_{D}$ is the voltage across the diode, $V_{T}$ is the thermal voltage, and $n$ is the ideality factor. $V_{T}$ is calculated by:

$$
V_{T}=\frac{k T}{q}
$$

where $k$ is the Boltzman constant, $T$ is temperature, and $q$ is the magnitude of charge. From here, changing the voltage will result in an exponential change in current. Because one focus is on forward bias characterization, a sample plot of the average I-V characteristics of several LEDs is show in Figure 2.9. These results were achieved by a lab characterization system. The I-V characterization module 
will be explained in detail in subsequent sections. The ability to measure the I-V characteristics of these LEDs will provide operational information such as turn-on voltages, devices resistances, and currents after the optoelectronic devices are exposed to the space environment. The methods by which these measurements will be used to give insight into damage that may be take place over time due to this exposure will be discussed later in this chapter.

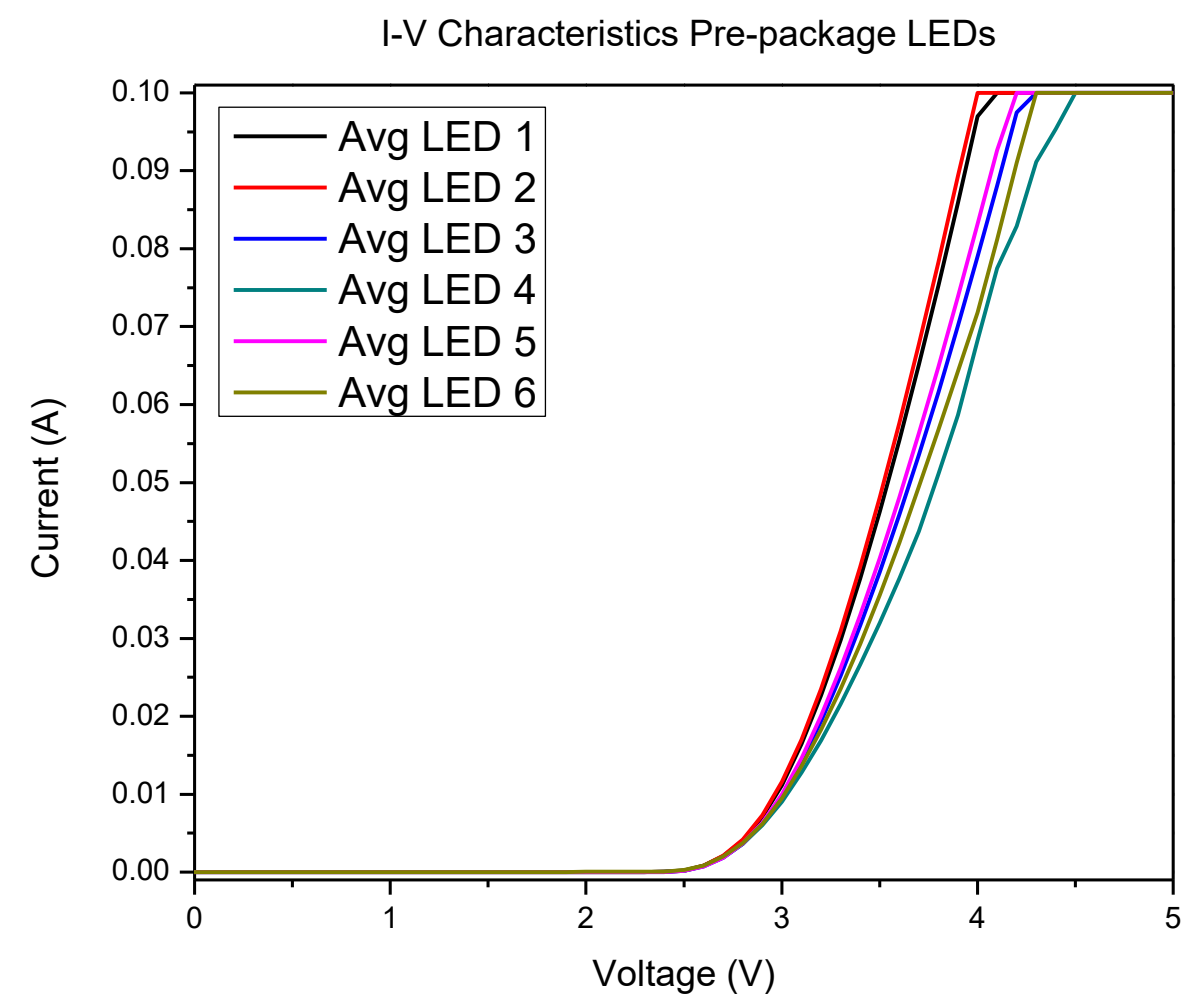

Figure 2. 9: Illustration of WVU LED I-V Characterization Curves

\subsubsection{Electroluminescent (EL) Characterization}

An electroluminescent (EL) response is created by radiative recombination of electron-hole pairs inside of a semiconductor material. A general representation of an EL curve is provided in Figure 2.10. As previously described, photodiodes are designed to be responsive to various wavelengths of light. They generate a very small current (nanoamps and or picoamps) as part of the photoconductive 
response of the photodiodes. Using this principle of operation, light output can be measured from LEDs to photodiodes. Additionally, because photodiodes can be responsive to certain wavelengths of light, this can be used to estimate the LED's emitted wavelength. Using these properties of photodiodes, LOCC will measure light intensity as well as estimate peak wavelength emissions.

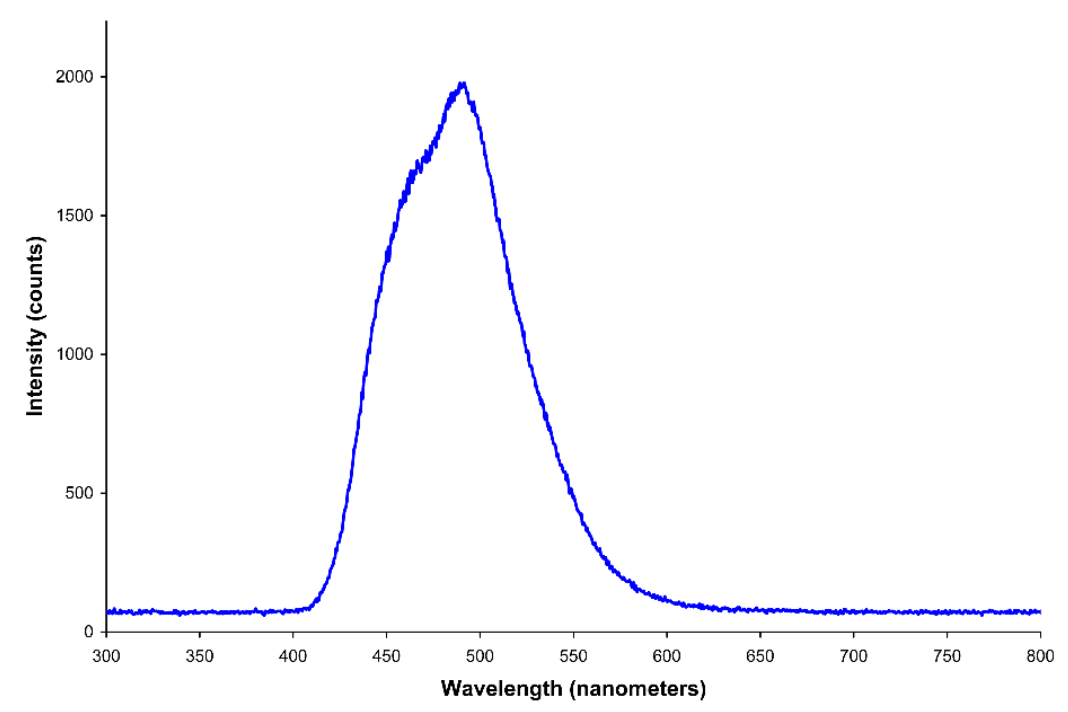

Figure 2. 10: Electroluminescent response of blue/green light source [146]

The LEDs were originally characterized using constant current methods at currents of $20 \mathrm{~mA}$, $40 \mathrm{~mA}, 60 \mathrm{~mA}$, and $100 \mathrm{~mA}$. The LEDs were characterized using a BWTEK Quest-Q CCD Array Spectrometer coupled with its software. Below, the average output of the pre-packaged LEDs at the mentioned currents can be seen. While monitoring the EL output of the LEDs, dependent variables such as light output and peak wavelength shifts can be measured. The results of the unpackaged LED measurements will also lend information to correction algorithms for determining the peak wavelength emission point. 
EL Characteristics Pre-Packaged LEDs

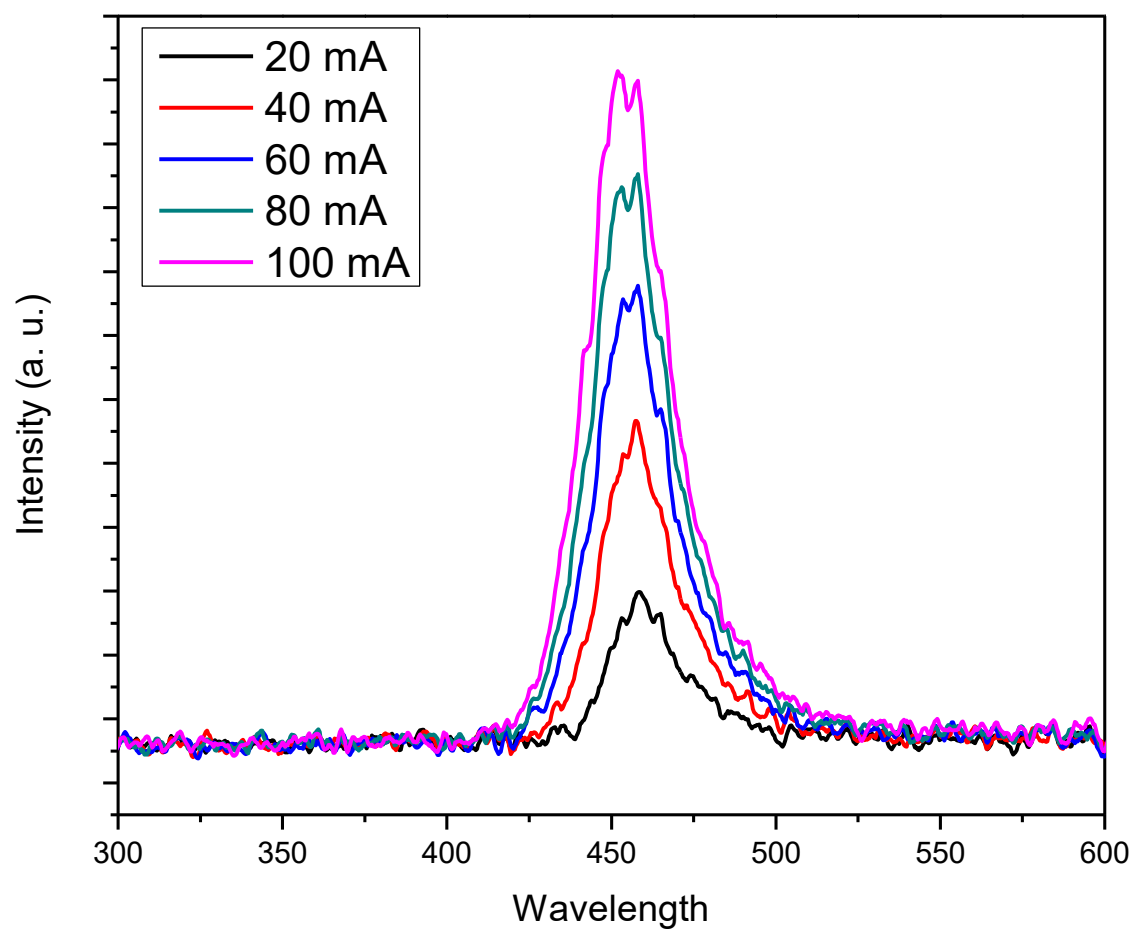

Figure 2. 11: EL spectral output of WVU LEDs

For LOCC to perform EL measurements of the LEDs, a MaZET MTCSiCF photodiode and ADC are used. The response of the MTCSiCF is based on CIE 1931 color space, which provides a link between wavelength and physiological perceived colors in the human eye [164].

Figure 2.12 illustrates the responsivity of the color matching functions with respect to wavelength. 


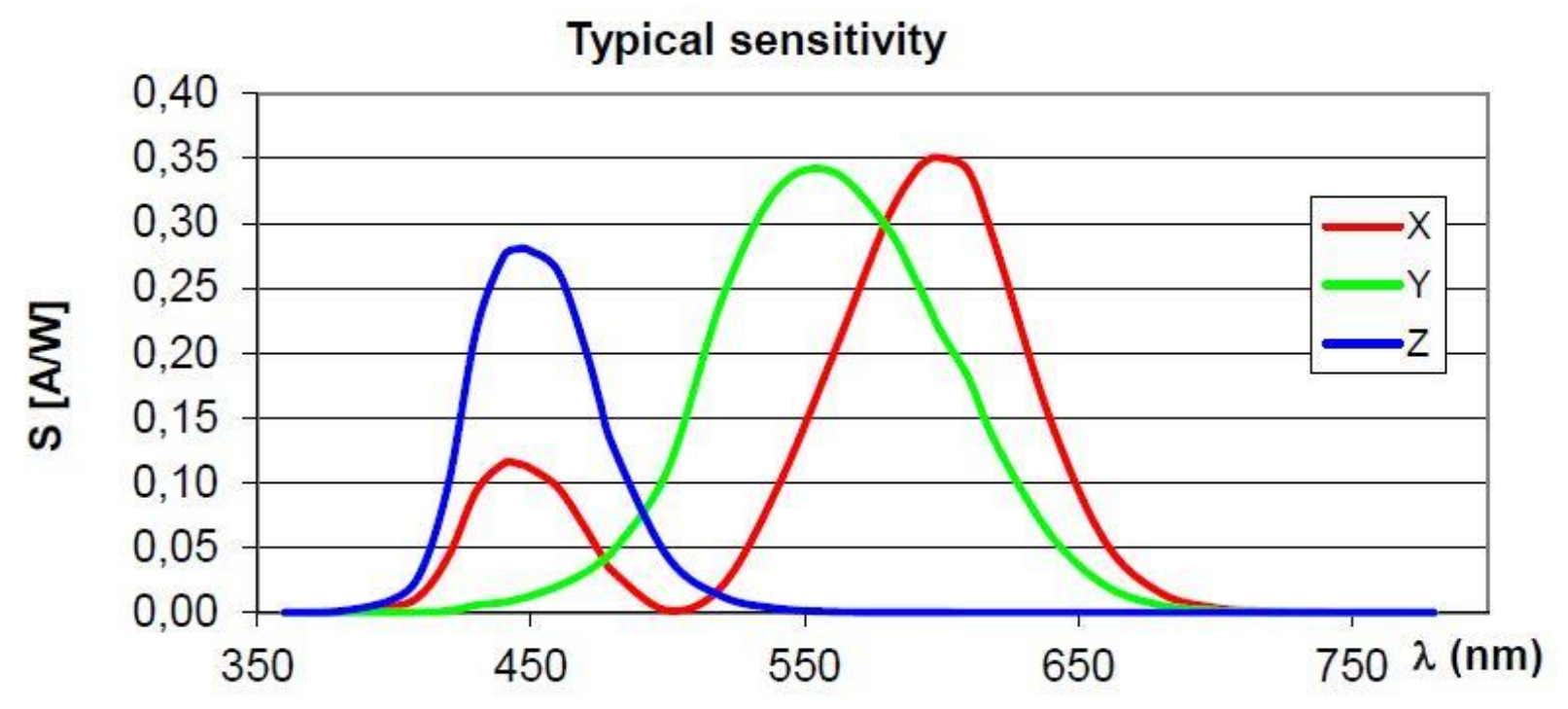

Figure 2. 12: Responsivity of XYZ color sensor MTCSiCF [163]

The tristimulus values are obtained by the relation of spectral radiance and the color matching functions:

$$
\begin{aligned}
& X=\int_{380}^{780} L_{e, \Omega, \lambda}(\lambda) X(\lambda) d \lambda, \\
& Y=\int_{380}^{780} L_{e, \Omega, \lambda}(\lambda) Y(\lambda) d \lambda,
\end{aligned}
$$

and

$$
Z=\int_{380}^{780} L_{e, \Omega, \lambda}(\lambda) Z(\lambda) d \lambda
$$

These values can then be passed into the CIE xy chromaticity diagram, and the CIE xyY color space where the peak emission wavelength can be interpreted, and the luminance of color can be mapped. Chromaticity of a color is specified by the two $\mathrm{x}$ and y parameters. These are normalized values being functions of all three tristimulus values $\mathrm{X}, \mathrm{Y}$, and $\mathrm{Z}$. The derived color space values $\mathrm{x}, \mathrm{y}$, and $\mathrm{z}$ are 
calculated from the tristimulus values. These can then be mapped into the CIE 1931 color space, seen in figure 2.13. The chromaticity equations are as follows:

$$
\begin{aligned}
& x=\frac{X}{X+Y+Z}, \\
& y=\frac{Y}{X+Y+Z},
\end{aligned}
$$

and

$$
Z=\frac{Z}{X+Y+Z}
$$

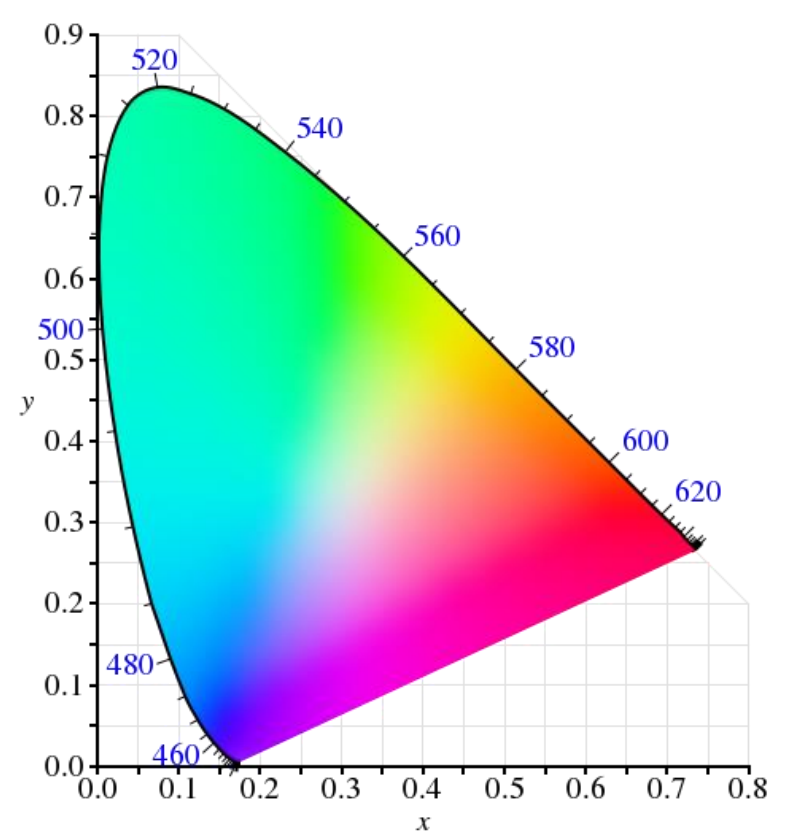

Figure 2. 13: CIE 1931 Chromaticity Diagram [165] [166]

It should be noted that, to achieve proper results the output values, the MCDC04 must be calibrated for applications for absolute color measurements based in the CIE 1931 standard. This is necessary for conversion into color space and helps compensate for opto-mechanical design variations. These values need to be adjusted for external effects such as temperature or other components within the 
system. A sensor calibration can be made by an individual, system or by an in-series calibration. Using an illumination source such as a monochromator and spectrometer as reference, this provides for the variables of the calibration. For calibration of the target, measurements by a spectrometer are known. By defining a coefficient matrix, the relationship between the measured sensors values and color coordinates in CIE 1931 color space can be made. T represents the matrix of the reference measurement. $\mathrm{S}$ is the sensor signal matrix, and $\mathrm{K}$ is the transformation matrix. After the transposition of S, a transformation matrix will be calculated, $\mathrm{K}$. $\mathrm{K}$ which is used to transform the measured sensor values into XYZ color space. The following equations show the calculation of correction matrix and the use of known spectrometer values:

$$
T=\left(\begin{array}{ll}
X 1 & X n \\
Y 1 & Y n \\
Z 1 & Z n
\end{array}\right), S=\left(\begin{array}{ccc}
x \operatorname{adc} 1 & x \operatorname{adc} n \\
y \operatorname{adc} 1 & y \text { adc } n \\
Z \operatorname{adc} 1 & z \text { adc } n
\end{array}\right)
$$

where $\mathrm{T}$ is the $\mathrm{Xn}, \mathrm{Yn}, \mathrm{Zn}$ of known $\mathrm{XYZ}$ measurements from a spectrometer, $S$ is ADC values from the MCDC04. The correction matrix $K$ is calculated by

$$
K=\left(T * S^{T}\right) *\left(S * S^{T}\right)^{-1}
$$

and

$$
K=\left(\begin{array}{ccc}
x k 1 & x k 2 & x k n \\
y k 1 & y k 2 & y k n \\
z k 1 & z k 2 & z k n
\end{array}\right)
$$


The calibrated output is defined as follows. Using the correction matrix, output values of the MCDC04 are found using:

$$
\left(\begin{array}{l}
X \text { sensor } \\
Y \text { sensor } \\
Z \text { sensor }
\end{array}\right)=K *\left(\begin{array}{ll}
x & \text { adc } \\
y & \text { adc } \\
Z & \text { adc }
\end{array}\right)
$$

where $\mathrm{X}, \mathrm{Y}$, and $\mathrm{Z}$ sensor are the CIE system values as corrected by the transformation matrix. This must be done to graph the output values to the xyY color space and chromaticity diagram. The values are stored in the LOCC system as the raw ADC values, and will need to be post processed after retrieval of the data transmitted from STF-1 while on orbit.

\subsubsection{Epoxies and Conformal Coatings}

It is important to note the use of transparent epoxies and conformal coatings in this research. Epoxies and conformal coatings used in spaceflight applications maintain integrity even after large temperature fluctuations. Conformal coatings can be used for multiple applications, including the protection of PCBs and electronic circuitry against particles such as dust, moisture, chemicals and temperature extremes [146]. It should be noted that ionic stopping properties have not been considered for certain conformal coatings, such as Parylene, remain transparent for temperatures upwards of $175^{\circ} \mathrm{C}$, with low visible region absorption, making it transparent for $455-465 \mathrm{~nm}$ wavelengths. It should also be noted that these conformal coatings can have radiation resistance towards gamma radiation in $100 \mathrm{Mr}$ dosages [148]. For examining LED light output for this project, it is assumed that the epoxies and conformal coatings do not severely impact the light output intensity, as they are listed at 99\% transparent against visible spectrum light. 


\subsection{Radiation Damage in InGaN/Gan LEDs}

Many environmental variables may have an effect on the operation of LEDs and the LOCC system while in space. The use of the LOCC system may aid in determining these effects on optoelectronics. It is important to note that the LED's minority carrier lifetimes are sensitive to atomic displacement within the material. The physical mechanisms of nonradiative recombination centers are introduced when under the bombardment of radiation. This effectively decreases the minority carrier lifetime. The decrease in minority carrier lifetime brings with it the mechanism of radiation-induced light output degradation. Nonradiative centers begin to out number radiative recombination centers, thus decreasing light output. Equations 2.18 - 2.28 have been derived in [136] and definethe initial carrier lifetime relating to light output of LEDs to the damage caused by radiation:

$$
\frac{1}{\tau_{o}}=\frac{1}{\tau_{o_{R}}}+\frac{1}{\tau_{o_{N R}}}
$$

where $\tau_{o}$ is the preirradiated minority carrier lifetime, $\tau_{o_{R}}$ and $\tau_{o_{N R}}$, respectively, are the radiative and nonradiative processes. Because nonradiative formations dominate the carrier lifetime, Equation 2.18 can be written as

$$
\frac{1}{\tau_{o_{R}}}=\sigma_{R} v_{t h} N_{R} \text { and } \frac{1}{\tau_{o_{N R}}}=\sigma_{N R} v_{t h} N_{N R}
$$

where $\sigma_{R}$ and $\sigma_{N R}$ are the carrier capture cross sections that respectively relate to radiative and nonradiative center. $N_{R}$ and $N_{N R}$ are the concentrations of the radiative and non-radiative centers, and $v_{t h}$ is the minority carrier thermal velocity. This allows the carrier lifetime to be written as the following, post irradition, 


$$
\frac{1}{\tau}=\frac{1}{\tau_{o}}+\sigma_{N R I} v_{t h} N_{N R I}
$$

In this equation, $\tau$ represents the postirradiated carrier lifetime and $N_{N R I}$ is radiated non-radiative centers and $\sigma_{N R I}$ is the radiated non-radiative carrier capture cross section. These centers are given by

$$
N_{N R I}=c_{i} \phi
$$

where $\phi$ is radiation fluence and $c_{i}$ is the defect density generated by unit of radiation fluence. The damage constant can now be defined. The damage constant $\mathrm{K}$ is written as

$$
K=\sigma_{N R I} v_{t h} c_{i}
$$

Note that $\mathrm{K}$ is a superposition of $\sigma_{N R I}$ and $c_{i}$, or a cascasde of damages, seen as

$$
K=v_{t h} \sum c_{i} \sigma_{N R I}
$$

The phenomenological equation to describe LED radiation damage can be written by substituting Equation 2.11 into 2.8, rearranged, and written as

$$
\frac{\tau_{o}}{\tau}=1+\tau_{o} K \phi
$$

Another note regarding the damage constant $\mathrm{K}$ is that $\mathrm{K}$ is determined through non-ionizing energy loss (NIEL) of radiation, the type of semiconductor material, its composition, and annealing that may 
be taking place while under temperature influences in space. This leads $\mathrm{K}$ to not be easily changed in order to achieve radiation hard LEDs. This does not mean that $\mathrm{K}$ cannot be used as a prediction device, as long as the limitations of this value are kept in mind. This leaves the lifetime-damage product, $\tau_{o} K$, the quantity of interest in finding due to its importance in determining the damages seen in LEDs.

For the LOCC system, the measurable values of light output and current as a function of forward voltage are needed to determine the damage constant. This means that the current controlling mechanism must be known. The LOCC system produces a constant voltage for the LED, leading to radiative current diffusion control. The resulting relationship of light output $I$ and minority carrier lifetime can bewritten as

$$
I=C \tau e^{\frac{q V}{k T}}
$$

The relationship between LED degredation and irradiation is modeled according to Rose and Barnes from [136]. It's given by the following rearrangment of Equations 2.13 and 2.12 as

$$
\left(\frac{I_{o}}{I}\right)^{\frac{1}{n}}-1=\tau_{o} K \phi
$$

Here, $I$ is the LED light output after irradtion, $I_{o}$ the the light output before irradiation, $K$ is a fitting paratermer, $\phi$ is particle fluence and $n$ is another fitting parameter dependent ondevice operating by diffusion current or recombination. For future analysis of equations for LEDs see [136]. GaN, K is $\sim 10^{-7} \mathrm{~cm}^{-2} s$ per particle of $40 \mathrm{MeV}$ protons [137]. The total current governed by space-charge recombination can be calculated similiarly to equation 2.14. Using the following equation [144]: 


$$
J=\frac{C}{\tau} e^{\frac{q V}{2 k T}}
$$

Where $C$ is a constant value, $q$ is the particle charge, $\tau$ is the carrier lifetime, $V$ is energy, $k$ is the Boltzman constant, and $\mathrm{T}$ is temperature. Similiarly, this can be rearranged as:

$$
\frac{J}{J_{o}}-1=\tau_{o} K \phi
$$

Degradation occurs when the value of $\tau_{o} K \phi$ approaches one.

\subsection{Thermal Effects on GaN LEDs}

Because temperature has a direct effect on EL spectra and I-V curves. This may lead to both a forward voltage descrease/increase and a peak wavelength emission increase with increasing temperature. The relation between junction temperature and forward voltage can be realized through the Shockley equation [138],

$$
J_{f}=J_{s}\left[e^{\frac{e V_{f}}{n_{\text {ideal }}^{k T}}}-1\right]
$$


where $J_{s}$ is the density of saturation current, $\mathrm{k}$ is the Boltzmann's constant and $n_{\text {ideal }}$ is the ideality factor. $J_{s}$. The ideality factor is expressed by

$$
J_{S}=e\left[\sqrt{\frac{D_{n} n_{i}^{2}}{\tau_{n} N_{D}}}+\sqrt{\frac{D_{p} n_{i}^{2}}{\tau_{p} N_{A}}}\right],
$$

where $D_{n}$ and $D_{p}$ are the diffusion constants of electrons and holes repespectively. The minority carrier lifetimes of electrons and holes are denoted as $\tau_{n}$ and $\tau_{p}$ respectively. The donor and accpetor energy state concentrations are denoted as $N_{D}$ and $N_{A}$. The intrinsic carrier concentration $n_{i}$ is expressed as

$$
n_{i}=\sqrt{N_{c} N_{v}} e^{-\frac{E g}{2 k T}}
$$

here $N_{c}$ and $N_{v}$ are the effective density of states at the conduction band and valence bands, repsectively.

For $V_{f}>>\frac{k T}{e}$, the original equation can be written as

$$
J_{f}=J_{s} e^{\frac{e V_{f}}{n_{\text {ideal }}^{k T}}}
$$

Solving for junction voltage,

$$
V_{f}=\frac{n_{\text {ideal }} k T}{e} \ln \left(\frac{J_{f}}{J_{s}}\right)
$$


The derivative of the forward voltage allows the determination of the temperature dependence of the given variables [139].

$$
\frac{d V_{f}}{d T}=\frac{d}{d T}\left[\frac{n_{\text {ideal }} k T}{e} \ln \left(\frac{J_{f}}{J_{S}}\right)\right]
$$

Substituing the previous equations in to 2.23 gives the temperature dependence of $n_{i}, E_{g}, N_{c}$, and $N_{v}$ . The derivative then yields

$$
\frac{d V_{f}}{d T}=\left(\frac{e V_{f}-E_{g}}{e T}\right)+\frac{1}{e}\left(\frac{d E_{g}}{d T}\right)-\frac{3 k}{e}
$$

Equations 2.35 shows the fundemental temperature dependence of forward voltage. The first sum on the right side of the equation is from the intrinsic carrier concentration, the second comes from temperature dependence of the band-gap energy. Because LEDs operate near to the built-in voltage, $V_{f} \approx V_{b i}$, this allows for nondegenerate doping concentrations to be written as

$$
e V_{f}-E_{g} \approx k T \ln \left(\frac{N_{D} N_{A}}{n i^{2}}\right)-k t \ln \left(\frac{N_{c} N_{v}}{n i^{2}}\right)=k T \ln \left(\frac{N_{D} N_{A}}{N_{c} N_{V}}\right)
$$

The band-gap energy can be written as

$$
E_{g}=E_{0}-\frac{\alpha T^{2}}{\beta+T}
$$

where $\alpha$ and $\beta$ are the Varshni parameters. $\alpha$ for $\mathrm{GaN}$ is $0.77 \mathrm{meV} / k^{2}$ and $\beta$ is $600 \mathrm{~K}$. Substituting the previous two equations, 2.36 and 2.37 , equation 2.35 can be written as 


$$
\frac{d V_{f}}{d T} \approx \frac{k}{e} \ln \left(\frac{N_{D} N_{A}}{N_{C} N_{v}}\right)-\frac{\alpha T(T+2 \beta)}{e(T+\beta)^{2}}-\frac{3 k}{e} .
$$

These equations expresses the temperature coefficient of the junction voltage, $V_{f}$. These equations and descriptions are used to understand the limitations of the LOCC system with regards to thermal regulation and the results of LOCC relating to the LEDs. Future design consideration should utilize know PCB thermal management techniques to midigate against thermal saturation.

For furthur junction temperature estimation, a forward voltage method can also be employed, though this requires a series of two measurments, a calibration measurement, and the actual junction temperature measurment. The calibration setup uses a duty cyle of $0.1 \%$ forward driving current, ensuring the junction temperature is the same as the ambient temperature. The calibration step establishes the relation between junction temperature and forward voltage. Last, an oscilloscope can be used to measure the forward voltage over various temperatures. The initial calibration step can be done prior to flight to see how the on orbit conditions are effecting the LED temperature. Previous experiments have shows that $V_{f}$ against $\mathrm{T}$ is close to a linear relation and can be fitted to [140]

$$
V_{f}=A+B T_{o}
$$

where $T_{o}$ is the oven temperature and $\mathrm{A}$ and $\mathrm{B}$ are fitting parameters. Following the calibration measurments, a forward current can be applied and the voltage values can be measured. The junction temperature can then be determined at various dc currents given by

$$
T_{j}=\frac{V_{f}-A}{B}
$$


Let it be noted that the LOCC system does not currently have the ability to perform these measurements, but the mechanics above can be used for future designs. These uncertainties that stem from the limitation of the LOCC system can be mitgated by providing temperature sensors and heatsinks to the LOCC sytstem to provide stable temperatures. Additionally, because the junction of LEDs are not able to be directly probed for temperature, the LED junctions conform to the temperatures of the system, PCB, or ambient temperatures. Ambient temperatures readouts will be given from parallel experiments onboard STF-1.

\subsection{Ion Stopping and Displacement}

There are two main systems that effect how a heavy ions and high-energy particles become trapped in GaN materials along with other crystal stuctures. These mechanisms are nuclear stopping and electronic stopping. Nuclear stopping occurs due to elastic collisions between the ion and nuclei in the lattice. This is important for low energies. At higher energies, particles are traveling too fast to have energy transfer. At low energies, nuclear stopping dominates the stopping power. The following equation represents the maximum stopping power within a crystal stucture.

$$
S_{n}=\frac{2.8 \times 10^{-15} M_{i} Z_{i} Z_{t}}{\left(M_{i}+M_{t}\right)\left(Z_{i}^{\frac{2}{3}}+Z_{t}^{\frac{2}{3}}\right)^{\frac{1}{2}}}\left(e V * c m^{2}\right)
$$

Oppositly, electronic stopping power dominates at high ion velocity, and maximum electronic stopping power is governed by the following: 


$$
S_{e}=\frac{\sqrt{\frac{Z_{i} z_{t}}{M_{i}^{3} M_{t}}}\left(M_{i}+M_{t}\right)^{\frac{3}{2}}}{Z_{i}^{\frac{2}{3}}+z_{t}^{\frac{2}{3}}} \sqrt{E}\left(e V * c m^{2}\right)
$$

In both equations $Z_{i}$ represents the atomic number of the ion, $Z_{t}$ represents the target lattice. $M_{i}$ is the mass of the incoming ion and $M_{t}$ is the mass of the lattice structure. The total stopping power is then decribed in Figure 2.8 and Equation 2.31. In electronic stopping, a "screen" of electrons will cause a significant drag on a passing ion. The resulting charge attraction creates a stopping force proportional to the ion velocity, described by

$$
S=S_{e}+S_{n}=\left(\frac{d E}{d x}\right)_{e}+\left(\frac{d E}{d x}\right)_{n}
$$

where $S_{e}$ and $\left(\frac{d E}{d x}\right)_{e}$ are the electronic stopping power, and $S_{n}$ and $\left(\frac{d E}{d x}\right)_{n}$ are the nuclear stopping powers. The two mechasism rely heavily on the mass of the ion and the mass and density of target. 


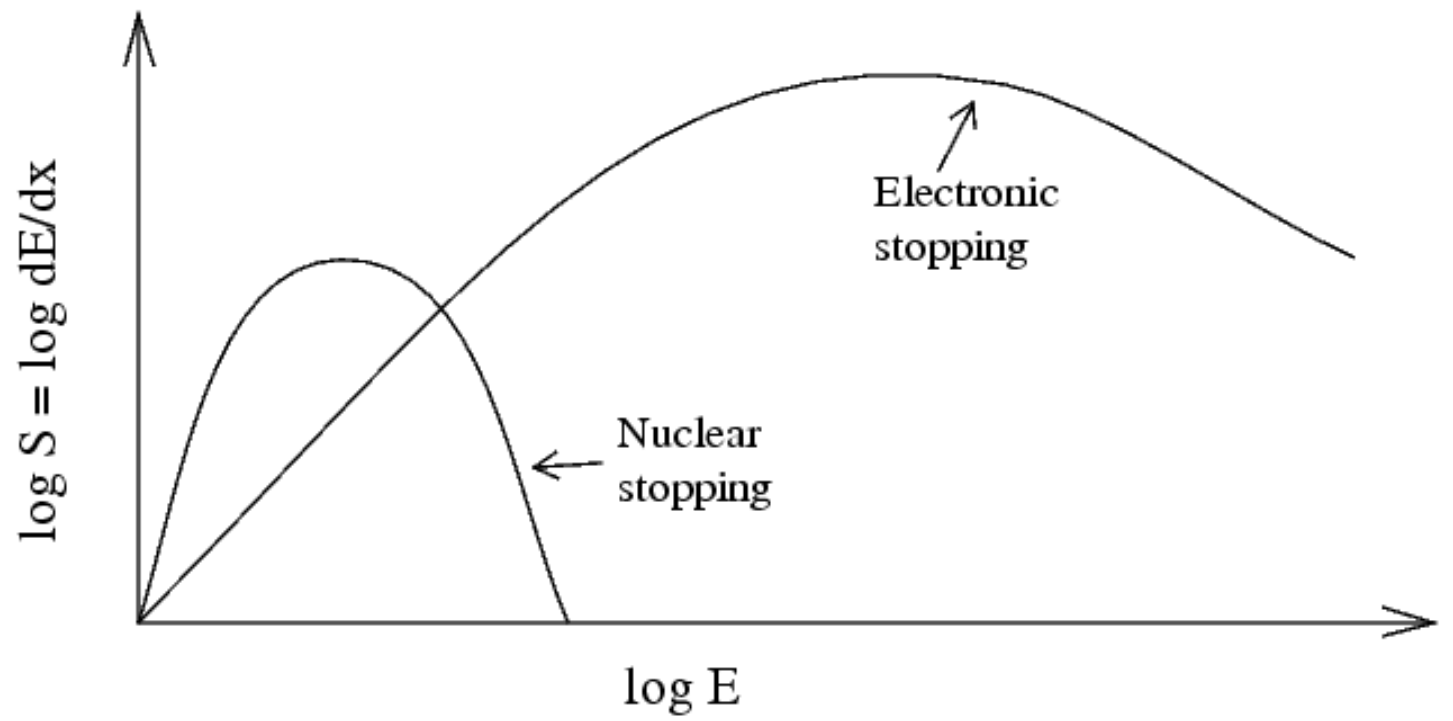

Figure 2. 14: Stopping power versus ion energy [143]

It is importatnt to note that models for ion implantation have been used rigerously and include the Monte-Carlo and Marlow [145 - 147].

For space applications, high-energy particles may pass right through the material. When this is not the case, these lower energy paticles will actually stop within the material. This gives a projected stopping range based on incident ion energy,

$$
N(x)=N_{p} e^{\frac{(x-R p)^{2}}{2 \Delta R_{p}^{2}}}
$$

where $N_{p}$ is the peak concentration, $R_{p}$ is the projected range, $\Delta R_{p}$ is the straggle, and peak concentration is $N_{p}=\frac{\phi}{\sqrt{2 \pi} \Delta R_{p}}$. With this dosing profile, different types of radiation will cascade their damage within the device structure and degredation will occur. This calls for the use of damage models with succesful displacement damage. It can now be seen how ionizing radiation affects our electronics. 


\subsection{Radiation Effects on Photodetectors}

Because photodectors are sensitive to dark thermal currents, a general design consideration is cooling the photodetectors to obtain correct results. Johnson-Nyquist noise, or the thermal noise relation, is given by

$$
i=\frac{4 k_{B} T B}{R_{C}},
$$

where $k_{B}$ is the Boltzman constant, $B$ is the bandwith if the device, $T$ is temperature, and $R_{c}$ is the device resistance. Because heating by radiation is the dominant factor, it should also be noted that the heat radiating from the Sun will generally effect results as well. Photodiodes will also experience a permanent increase in leakage current due to displacement damage. For this, a calculation can be performed to accurately describe the dark current increase. The approach can be used for radiationinduced dark currents which produce generation centers [141]. The equation for dark current density change can be written as

$$
\Delta J_{d}=\frac{q n_{i} x_{d} \phi}{2 K} .
$$

Where $n_{i}$ is the instrinsic carrier concentration, $x_{d}$ is the depletion region width, $\phi$ is the particle fluence, and $\mathrm{K}$ is the damage coefficient. For calculations regarding proton and electron bombardment proper damage coefficients see [142]. 


\subsection{Summary}

In summary, there are many environmental variables that may impact the operation of the LEDs and the LOCC system while under the conditions of the ionosphere. With the knowledge of both the LED structures and the effects of radiation damage, the use of the LOCC system shows potential in determining the effects of space on optoelectronics. This chapter has examined the fundamental operations of LEDs, their crystal structure, their engineering principles, and how these values relate to emission and absorption. For examining the damage caused by radiation, a damage coefficient can be found from the relationship of minority carrier lifetimes pre and post irradiation.

Thermal and annealing effects will also take place. This needs to be considered for system purposes, as providing a thermally-stable system ensures that characteristics that are being observed and not due to unforseen environmental conditions. Junction voltage is directly related to temperature and, therfore, peak emission and turn-on voltage will change due to environmental effects. It is suggested that analysis of the fabricated LED be examined over temperature extremes to gain a sense of how temperature will effects peak wavelength emission and I-V characteristics. Alleviating uncertainties in the LOCC can be with utilizing telemetry given by STF-1 and its subsequent experiments. Table 2.1 provides an overview of the damage parameters and what LOCC will determine based on the provided information. LOCC can not determine junction temperature, but methods have been mentioned for future work. 


\begin{tabular}{|c|c|}
\hline \multicolumn{2}{|c|}{ Table 2. 1: Damage Parameters and Used Equations } \\
\hline Damage Parameter & Equations \\
\hline constant & $\left(\frac{I_{o}}{I}\right)^{\frac{1}{n}}-1=\tau_{o} K \phi$ \\
\hline Peak Wavelength emission shift & $\left(\begin{array}{c}X \text { sensor } \\
\text { Y sensor } \\
Z \text { sensor }\end{array}\right)=K *\left(\begin{array}{l}x \text { adc } \\
y \text { adc } \\
z \text { adc }\end{array}\right)$, \\
Threshold Voltage Shift & $x=\frac{X}{X+Y+Z}, y=\frac{Y}{X+Y+Z}$ \\
\hline Series Resistance Change & LOCC output I-V Characteristics \\
\hline LED Junction Temperature Estimation & LOCC output I-V Characteristics \\
\hline Current Density & $T_{j}=\frac{V_{f}-A}{B}$ \\
\hline
\end{tabular}

The physical mechanisms for the displacement and penetration of high energy particles found in the upper atmosphere are examined. This gives insight into how the particles are projected into the materia,l and how stopping power may be tailored by changing materials or material properties. The final portion of this chapter looked at the effects of irradiation of photodetectors and how thermal noise changes the outcome of results. This leads to a need for radiation tolerent photodiodes as well as thermal stability to produce accuarate results with the LOCC system. 


\section{Chapter 3: System Design and Experimental Test Plan}

\subsection{Introduction}

This chapter will provide details on the design of the LOCC experiments, including the power and space requirements of the CubeSat platform, data requirements of the flight computer, and device requirements for on-board LED characterization. A general hypothesis is stated regarding the relations between fluence, carrier lifetime, and light intensity outputs. This chapter will consider characterization parameters that have been established from the literature review. Following this, a discussion of the overall system operation and data generation are provided.

Based on the literature review, optical and electrical properties are of interest and will be examined via the LOCC system. Dependent variables such as light intensity, turn-on voltage, wavelength shift, and saturation currents will be observed and compared over time while under the influences of total ionizing dose (TID) and displacements damages. Based on [144], the measurable values that will be used to characterize our devices will be light output and current as a function of forward bias. In Equation 2.14, the damage constant $\mathbf{K}$ describes the physical mechanisms. At constant voltages the variable of interest is the initial lifetime-damage constant product, $\tau_{o} K$. Using the following equation from [144]:

$$
\frac{I_{o}}{I}=1+\tau_{o} K \phi
$$

it can be observed that the values of degradation under constant voltage will fall between 1 and 2 as $\tau_{o} K \phi$ approaches unity, signifying large damages. It has been previously reported that optical degradation will occur, and a decrease in light output over fluence will be observed while an increase 
in current is also observed; stemming from deep level trap formation and resulting in non-radiative recombination.

\subsection{System Overview}

The design and layout of the LOCC system is based on the size constraints of the PC104 specification, and power and data limitations of the CubeSat platform [149]. The figures included in this section will illustrate how the LED component and photodiode component boards of the LOCC system will fit together. It also illustrates where LOCC will be located within the $3 \mathrm{U}$ stack of theSTF1 system. Figure 3.1 shows the system overview. The design of LOCC allows for future designs to scale based on the number of electronic devices or size of the satellites. A stacked configuration is utilized to spatially align the LEDs and photodiodes. LOCC is comprised of two main PCBs. Both PCBs are governed by four major hardware modules that include: power management, communication isolation, LED I-V characterizer and microcontroller, and the electroluminescence characterization unit. This section will give an overview of the LOCC system and how its sub-modules will be used to accomplish LED characterization tasks.

\subsubsection{Identification}

Table 3.1 gives an overview of abbreviation, title, version, and release of the LOCC system. This should be used to alleviate any discrepancies between future models and revisions of the LOCC system. For future renditions, such as a desktop version of the LOCC system, abbreviation can be

changed to suite the experimental environment. An example would be, to recreate this system for desktop, LOCC can be renamed to a Low-powered Optoelectronic Characterizer for Desktop (LOCD). 
Table 3. 1: System Identification

\begin{tabular}{|l|l|}
\hline System Title & $\begin{array}{l}\text { Low-powered Optoelectronic } \\
\text { Characterizer for CubeSats }\end{array}$ \\
\hline Abbreviation & LOCC, LOC-C \\
\hline Version & 1 \\
\hline System Address & $0 \mathrm{x} 08$ \\
\hline
\end{tabular}

\subsubsection{Module Overview}

LOCC consists of a modular design for ease of modification and individual operations. This allows for LOCC to be modified and scaled to meet data and power constraints based on the desired experiment. Figure 3.2 illustrates the block diagram of the LOCC system interconnections and the connections from the flight computer. In this case, STF-1's on-board computer provides LOCC with command operations, and can receive telemetry to be sent back to the ground station.

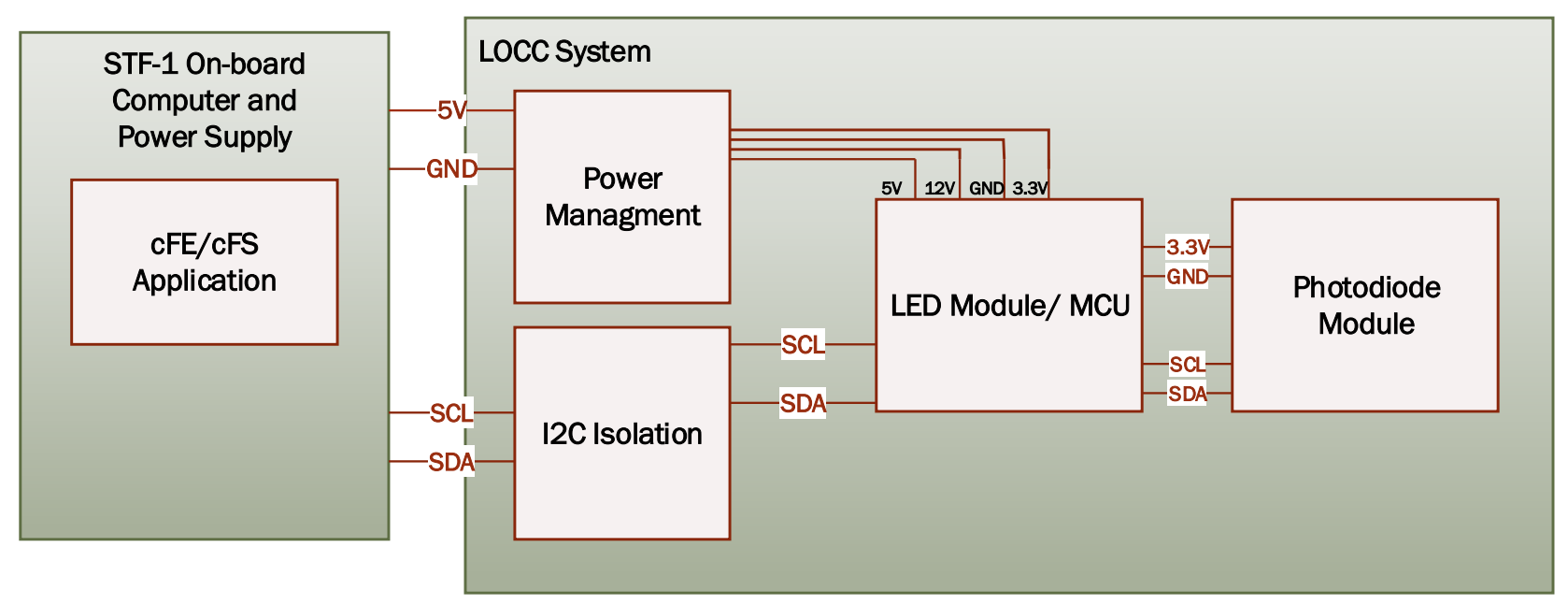

Figure 3. 1: Block Diagram Illustrating the LOCC system and embedded modules [155] 


\subsubsection{LED Characterization/MCU module}

Figure 3.2 shows the block diagram of the LED Characterization Module. The LOCC master controller is based on the Arduino Mega 2560 [156]. The Arduino-based firmware allows for configuration of switching, voltage ramping, voltage sensing, data management, and master/slave I2C communication.

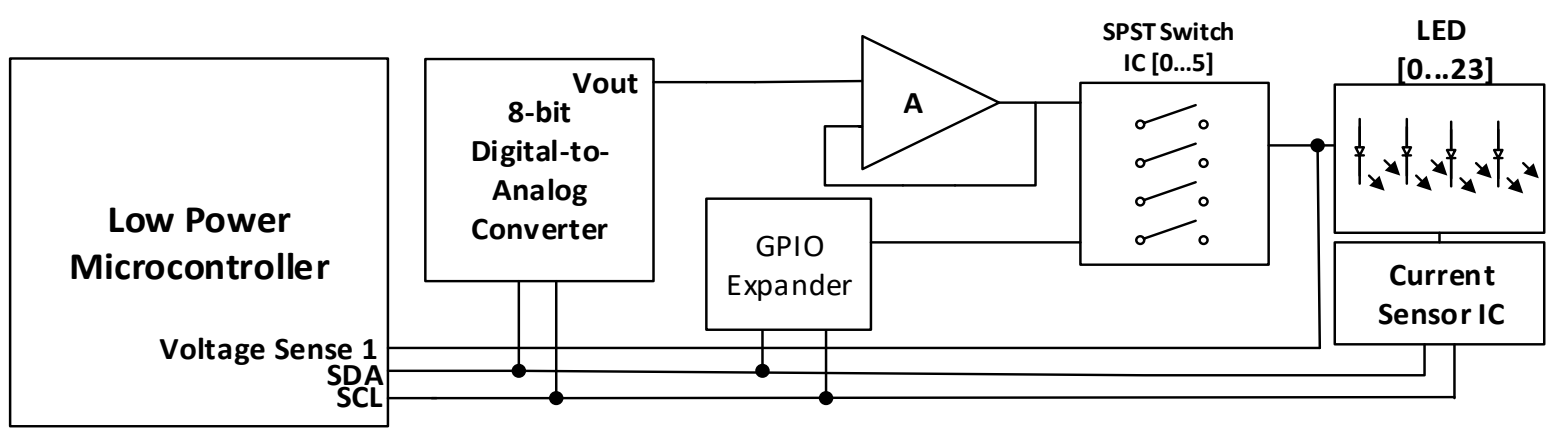

Figure 3. 2: Block Diagram of the LED Characterization Module

The MCU and its firmware allows for direct communication with STF-1's flight computer for data collection and transmission. To achieve the $0 \mathrm{~V}$ to $5 \mathrm{~V}$ voltage ramp needed for LED I_V characterization, an 8-bit digital-to-analog (DAC) converter is used. A Microchip MCP4901 uses a SPI communication protocol to provide a continuous voltage level with $\mathrm{mV}$ accuracy and thermal stability [157]. This device provides the necessary voltage level, but cannot supply high amounts of current. In this case, the DAC is connected to a unity gain operational amplifier (opamp) that is designed to supply a linear current output. Analog Devices manufactures an opamp with exceptional current supply values (150 mA to $375 \mathrm{~mA})$. The AD8397 provides up to $310 \mathrm{~mA}$ peak linear outputs and can drive heavy loads such as the current demand on an LED [158]. It should be noted that a current limiting resistor should be incorporated to avoid burning or shorting the opamp. The opamp is configured with $12 \mathrm{~V}$ supply to operate within desired range and allows enough current to be sourced 
for operation. Because the module is designed with scalability in mind, redundancy is provided with the use of multiple LEDs and single-pole-single-throw (SPST) switches. Analog Devices makes an IC that is used in data acquisition systems and allows for high current to pass through ( 200 mA) [159]. The use of SPST switches offers control over which LED is turned on at any given time. This is crucial in performing voltage ramping. Activating more than one LED at a time may pull too much current and damage or destroy the subsequent components. Because the SPSTs are based on CMOS technology the selection of source and drain channels is dependent on logic level controlled by GPIO expanders. The Microchip MCP23018 is configured to supply the proper logic levels to ensure the correct switching [160]. For current sensing, a Texas Instrument (TI) INA219 current sensor is used [161]. The LEDs are attached to the INA219 using a shunt resistor configuration of $0.1 \Omega$, and the voltage change over this resistor is measured to determine current. Using this device, a mA-accurate reading of the current passing through the LED can be read and stored in the MCU. Separation of internal I2C and the onboard computer I2C communications is needed. This allows for interrupts to be sent while experiments are running. To achieve this separation of communication, LOCC uses the ATMEGA's ability to modulate digital pins and mimic I2C communication. This technique is referred to as 'bit banging,' and it can provide a $100 \mathrm{MHz}$ signal to all I2C connected devices.

\subsubsection{Electroluminescence Characterization Module}

A similar approach is used for characterizing the LEDs and their electroluminescence. For the case of the EL side, a combination of components is used to allow the photodiode sensors to be activated using I2C interfacing. MAZe'T Electronic Engineering \& Manufacturing Services produce a 4-channel analog-to-digital converter (ADC) with I2C control and output, the MCDC04 [162]. 


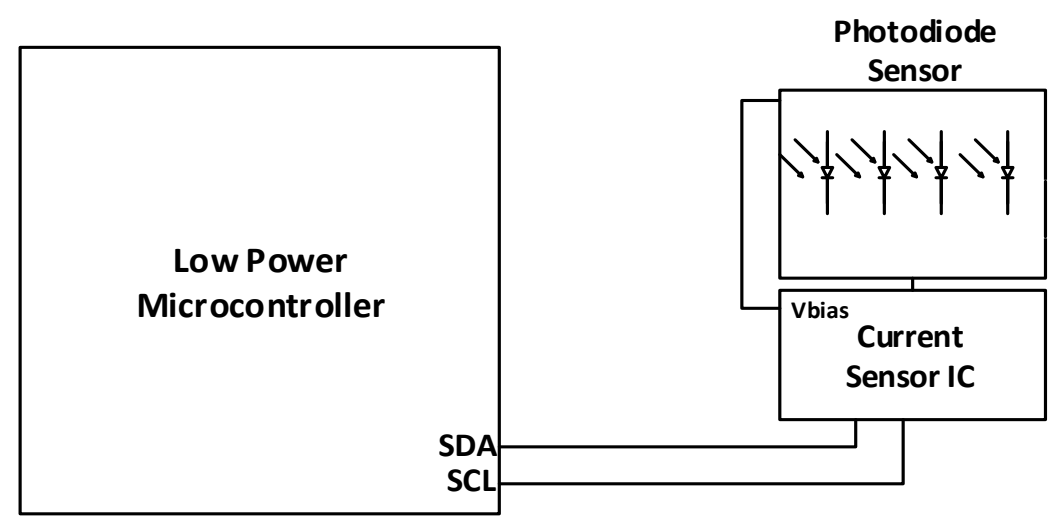

Figure 3. 3: Block Diagram of the EL Characterizer

This component measures small current changes that happen in silicon PIN photodiodes. The MCDC04 provides 16-bit signal resolution and is configurable to $20 \mathrm{fA} / \mathrm{LSB}$ sensitivity. This IC supports multiple forms of operation that include:

- $\quad$ CMD Mode - For single measurement and conversion (I2C controlled)

- CONT Mode - Continuous measurement and conversion until told to stop

- $\mathrm{SYN}[\mathrm{x}]$ modes - Synchronized with external source

- SYNS Mode - Synchronized at Start

- SYND Mode - Synchronized at Start and Stop measuring cycles

This IC allows the user to set current ranges and integration time for varying applications. Utilizing control of the ADC allows for controlled power usage, as the devices can be sent into Power Down and Standby modes, making it suitable for mobile solutions. Because the converter is highly flexible, it allows for a wide range of usage in lighting applications, such as the measurement of intensity and color coordinates (XYZ CIE 1931, DIN 5033, etc). 
The MTCSiCF True Color Sensor is based on CIE 1931/DIN 5033 (human perception) [163]. The JENCOLOR, housed within the MTCSiCF, sensor is comprised of 19x3 photodiodes in silicon PIN configuration, allowing for sensitivity and speed, and operates within $-20^{\circ} \mathrm{C}$ to $100^{\circ} \mathrm{C}$. Operation outside of these temperatures may result in filter shifting and erroneous data.

\subsubsection{Power Management}

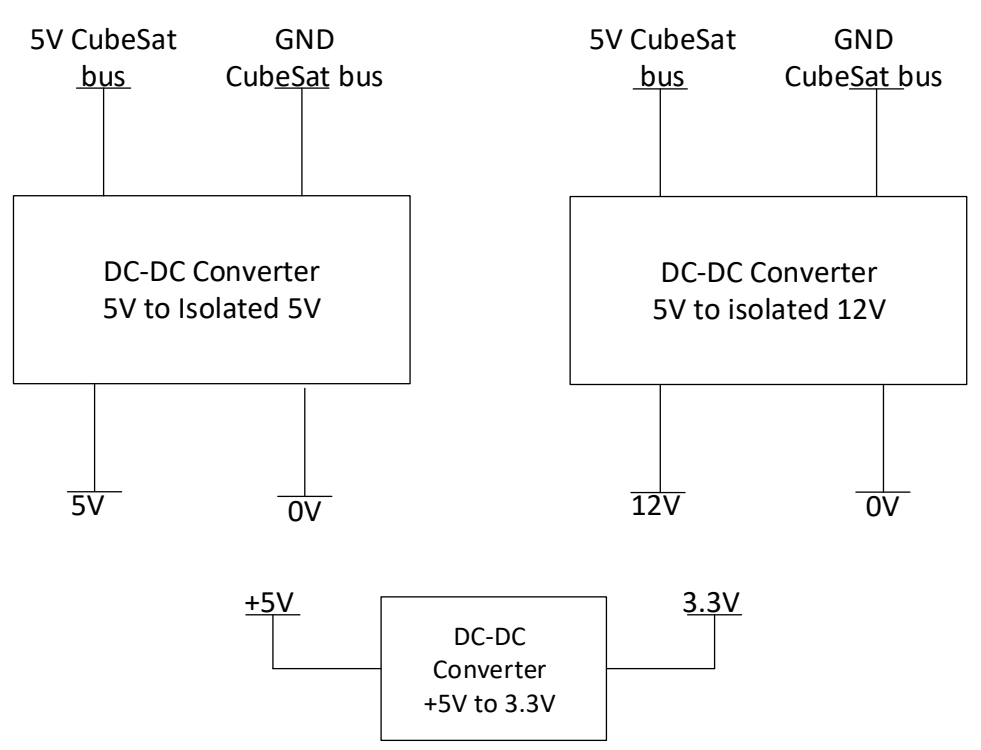

Figure 3. 4: Block Diagram of the Power Management module

The power management module is fundamental to the operation of the LOCC system, as it supplies up to $4 \mathrm{~W}$ of total isolated power with an efficiency of up to $82 \%$. This is done using the CUI INC PDS2-M DC-DC converter [167]. Isolated 5V and 12V voltage levels are generated with a common 0V. From the 5V supply, a $3.3 \mathrm{~V}$ is generated for logic level conversion and other circuit components requiring lover supply voltage. This provides LOCC with power supply lines that are dedicated to MCU and processing ICs, and leaves a separate $12 \mathrm{~V}$ supply to provide current to the LEDs. Because the bus power supplied to the experiment is susceptible to fluctuations in the line, it 
is necessary to supply isolated power and provide protection to overcurrent draw to the experiment. These DC-DC converters are ideal to provide these safety mechanisms, and give design restriction to operate within $5 \mathrm{~W}$. The converter connects the inner power and ground planes of the LOCC I-V Characterizer PCB. The $12 \mathrm{~V}$ supply is used to manage voltage ramping to ensure the LEDs can run from 0 to $5 \mathrm{~V}$. This ensures stable operation during LED ramping and power supply to other components, and maintains a lower demand. Power management also need to provide logic level transitions and a 3.3V supply to other devices. This is done using the MICREL MIC5225 Regulator [168]. This IC provides a stable voltage output while under small loading effects. The calculated wattage for the operation of the LOCC system is listed below.

Table 3. 2: Power Budget of LOCC system

\begin{tabular}{|c|c|}
\hline \multicolumn{2}{|c|}{ Power Budget } \\
\hline Total (TYP) (W) & 1.397 \\
\hline Total (MAX) (W) & 4.757 \\
\hline
\end{tabular}

\subsubsection{I2C Interfacing Module}

LOCC communicates via I2C communication. This is done for to meet STF-1's 100kHz I2C communication requirements. Keep in mind that LOCC operates with two separate I2C buses. One being the 'bit bang' bus that communicates with the systems components, and the other being the system I2C bus which allows LOCC to talk to the master computer. To meet the STF-1 communication requirements, an I2C interface module is implemented into the LOCC system. Figure 3.5 describes the block diagram of the $\mathrm{I} 2 \mathrm{C}$ isolation. 


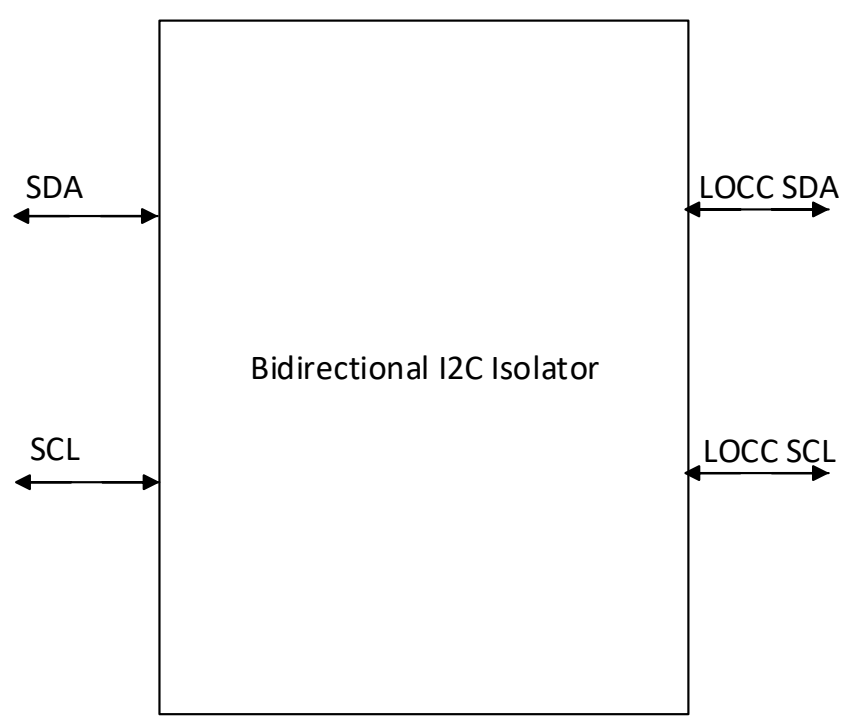

Figure 3. 5: Block Diagram of I2C isolated Module

Similarly, the communication bus is also susceptible to unstable voltages and other uncertainties in the line. For this, the I2C module provides the needed protection for LOCC's communication from the master computer to the MCU. This is done with a Texas Instrument ISO154x capacitive isolator [168]. It should be noted that the original schematic design involved three components rather than one for level translation and signal integrity. Due to previous schematic design, an optocoupler was installed but incorrectly configured. This led to a modification using the ISO1540. For this, the device was placed on unpopulated pads and connected via hand-soldering to the necessary pins. For future design consideration, the same modification can be implemented in layout, or the modification can be performed. 


\subsubsection{Installation of modules}

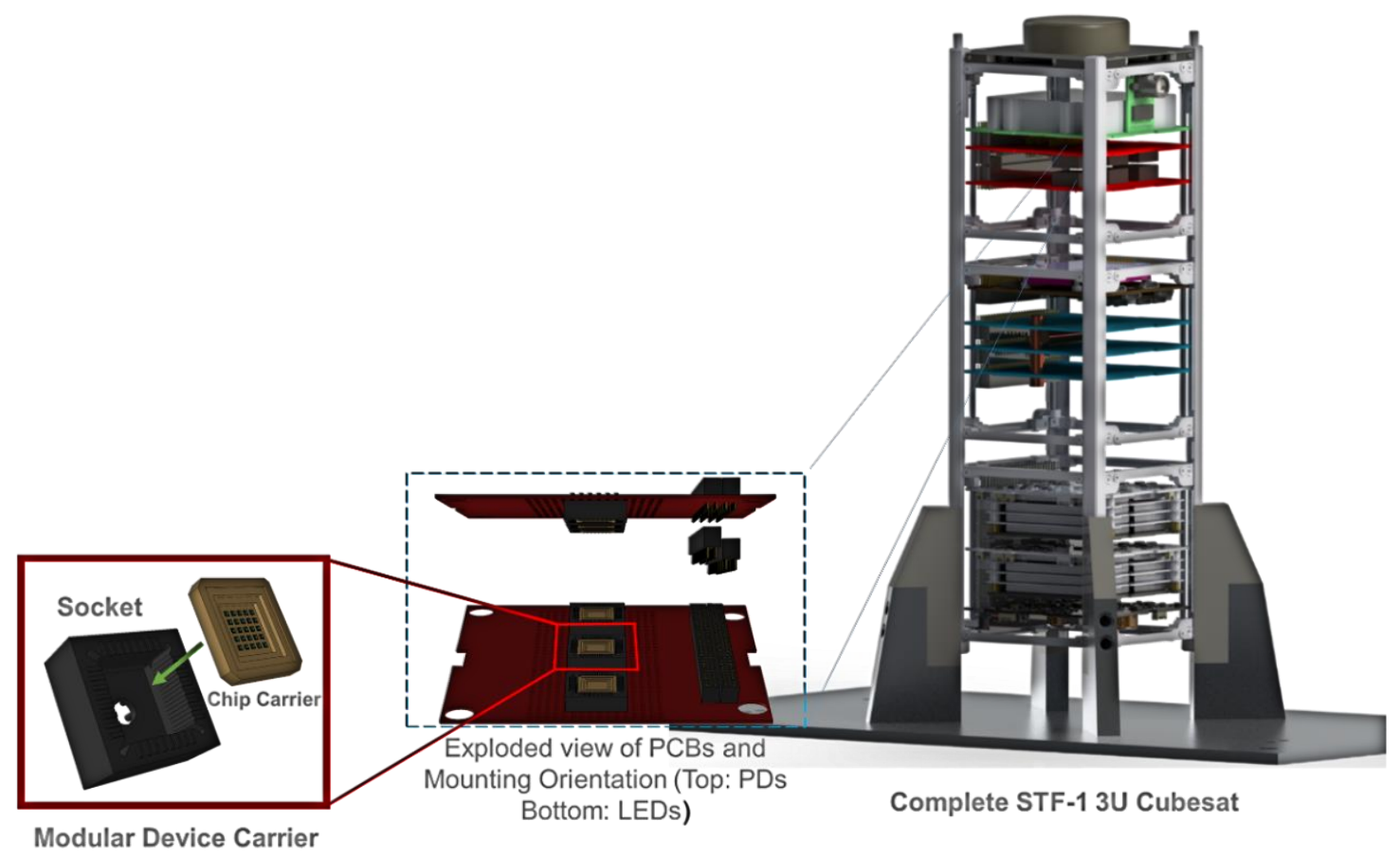

Figure 3. 6: (From Left to Right) Chip Carrier and Chip Carrier Socket, Photodiode and LED PCB modules (exploded), STF-1 CubeSat Rendering without Solar Panels

Image 3.2.1 illustrates the three main components of implementing the LOCC system. Starting from left and moving right, the LED carrier and socket are shown. Within this module. the LEDs are packaged into a chip carrier and seated into the socket. The chip carriers and chip carrier sockets are detailed in [150] [151], and correspond to the 3 leadless chip carriers (LCC) models. Following the device carrier installation comes the LOCC system, which houses the LEDs and, will be the source of control for I-V characterization and electroluminescent characterization. The systems modules are internally connected via male and female header pins produced by Harwin, respectively [152] [153]. 
The cross-sectional in Figure 3.7 shows the dimensions of the system when aligned and assembled for interconnection.

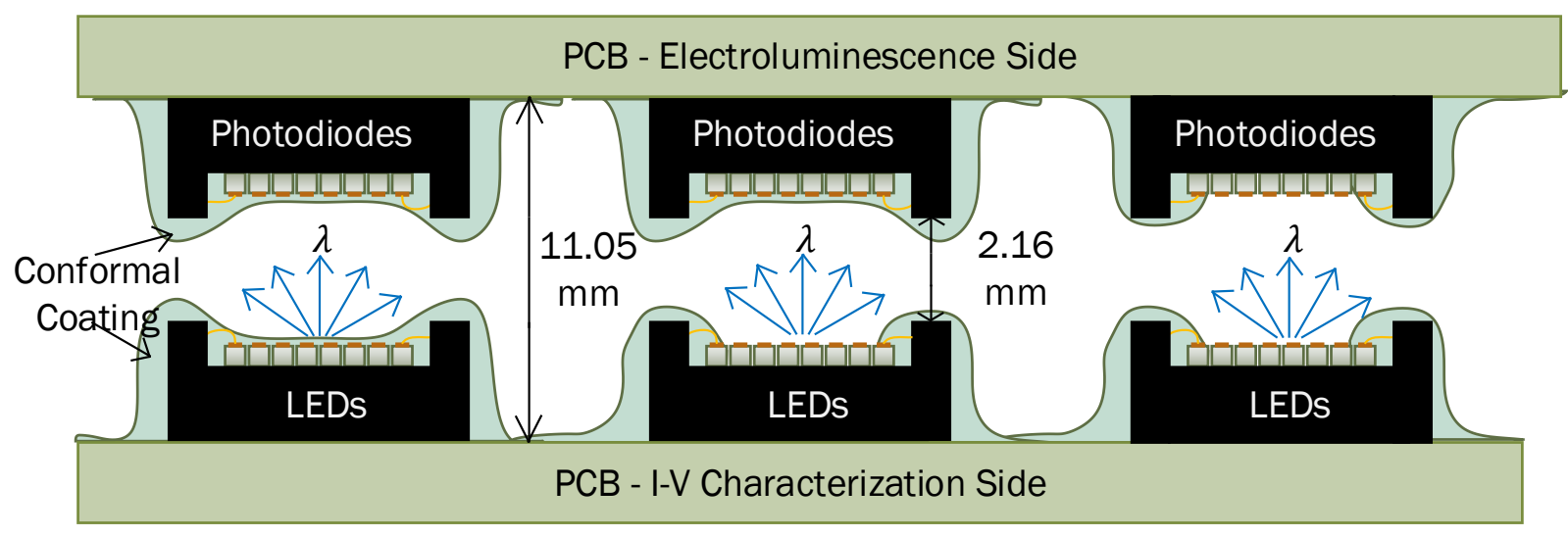

Figure 3. 7: Cross-section of connected LOCC system

When testing the LEDs and their respective photodiodes in space, it is important to establish a control group of devices to accurately interpret the results of the experiments. A layer of transparent conformal coating will be added to the boards comprising the LOCC system. This conformal coating will give us a degree of shielding to minimize the potential for damage to the control devices. Three pairs of LED/PDs will be used, as seen in Figure 3.7. On the left of Figure 3.7, Set A will consist of both LEDs and PDs being coated. The middle Set B array will consist of coated PDs and uncoated LEDs. For Set $\mathrm{C}$ on the right, both LED and PD devices will remain uncoated. A comparison of the uncoated and coated devices will allow for the effects of the space environment on the unprotected InGaN LED devices to be monitored via device characterization methods.

The LOCC system sits within a $1 \mathrm{U}$ CubeSat configuration and is powered by the CubeSat; formed by the connecting header pins that span the length of the CubeSat chassis. Within the pin configuration of the header pin, LOCC sends and receives information. Samtec manufacturers a variety of PC/104 specific connectors with different widths, lengths, height, and connection type. For 
the CubeSat specification, the Samtec ESQ-126-39-G-D was chosen from [154]. The connections on these header pins should match mission requirements, and caution should be used to make sure that there are no shorts or missed connections to ensure proper communication with the flight computer. Figure 3.8, 3.9, and 3.10 show the connection type for board-to-board interfacing.

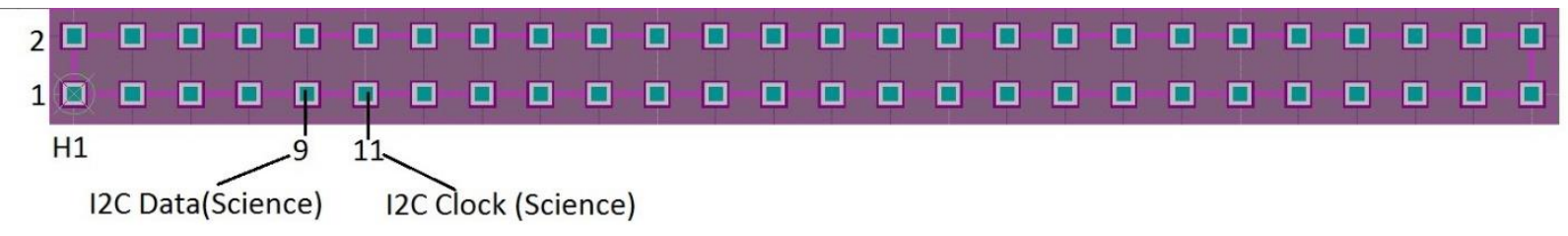

Figure 3. 8: (Top Down View) LOCC Header Pin 1 for STF-1 connection

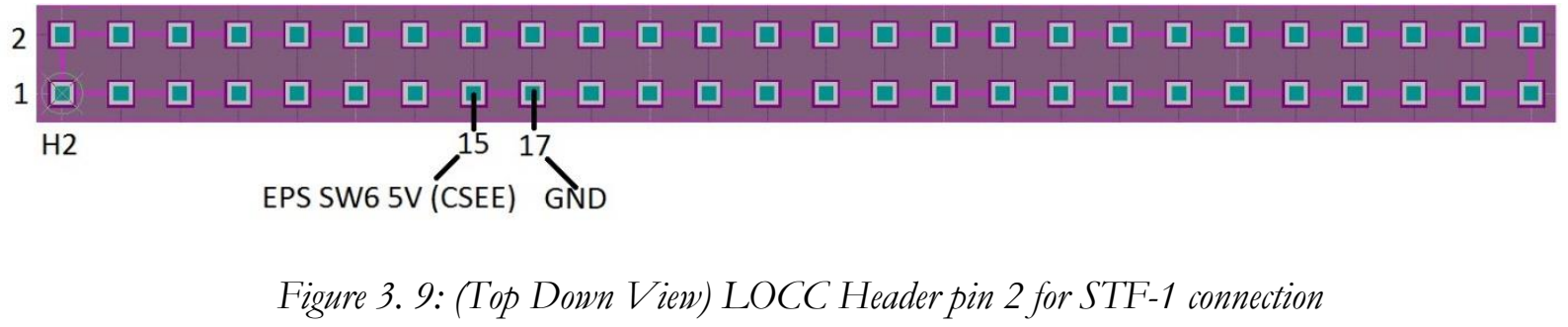

The modules can be "sandwiched" together to close the connection and allow communication between the microcontroller unit (MCU) and EL side. It should be noted that both can be used individually. 


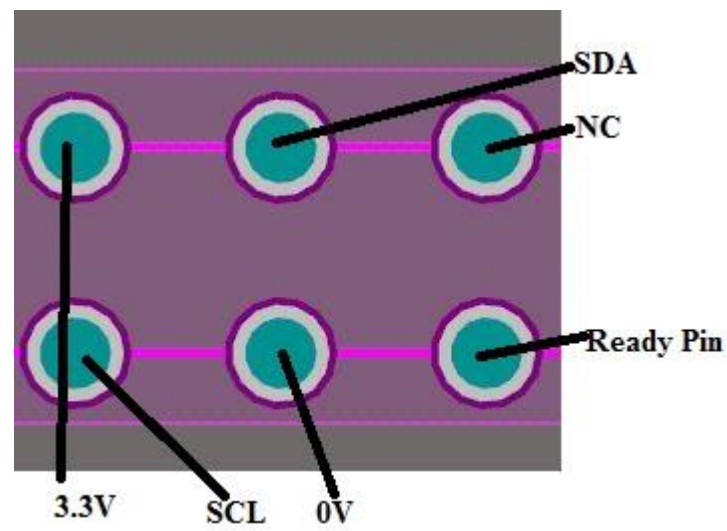

Figure 3. 10: (Top Down View) LOCC inter-board connection

Figure 3.11 illustrates a side-view of the two boards being pressed together to complete the connection. Once the boards are attached, the user can then make the proper connections to the ESQ126-39-G-D including power, ground, and I2C data.

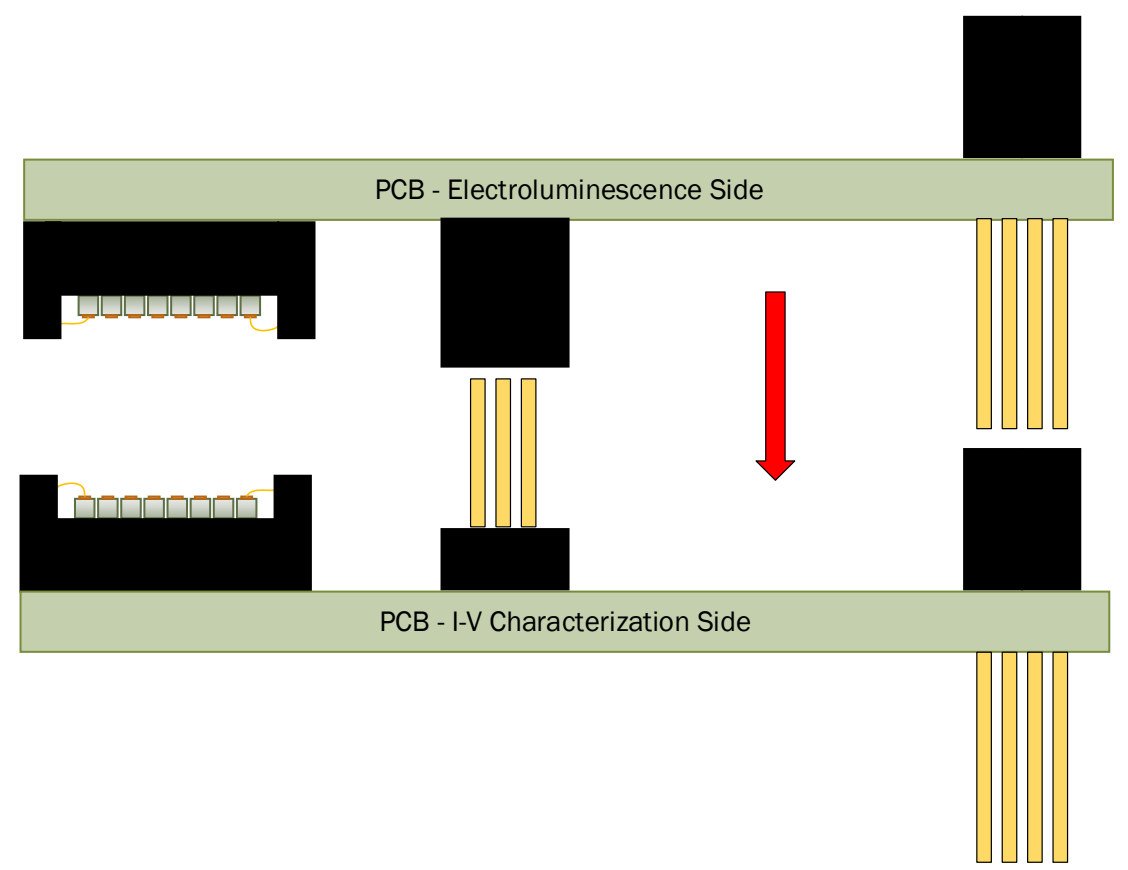

Figure 3. 11: Side profile of connection diagram for assembly of LOCC 
The LOCC system is controlled by the flight computer via inter-integrated circuit (I2C) commands. For STF-1, the control is done by Core Flight Executive (cFE,) a software application that sends hexadecimal values to read and write data to and from the LOCC system. For initial prototyping, LOCC is controlled by both Arduino MEGA 2560 and Raspberry Pi v.2 using C and $\mathrm{C}++$ command programs. Details regarding protocol, and embedded firmware can be found in the Design sections. Data is stored as hexadecimal values, and will need to be parsed out based on given byte values for data packets in the firmware.

\subsection{Circuit Schematics of LOCC Modules}

For this section, the circuit diagrams will be explained regarding the modules of the LOCC system. This will provide a look at what components are used, as well as the details regarding resistors, capacitors, diodes, etc. This section begins by looking at the MCU/I-V Characterization module of LOCC, followed by the EL circuit schematic. This will give an idea of how the components are connected for future maintenance and modifications. Looking further at the modules, the I2C circuit and power management circuits illustrate how LOCC communicates to the flight computer as well as how it is powered. The full circuit diagram can be seen in Figure 3.12.

\subsubsection{MCU/I-V Characterizer}

Figure 3.12 illustrates the complete MCU/I-V Characterizer and Figure 3.13 shows the ATMEL ATMEGA 2560 microcontroller unit (MCU) [169]. This was originally based on the Arduino MEGA 2560 schematic and modified to fit within the PC104 PCB Specification. The ATMEGA 2560 is the master component of the LOCC experiment. Communication with the CubeSat flight computer is handled by this component, as well as communication with the various components of the LOCC experiment. From the MCU, the DAC, current sensor, GPIO expanders, and EL's ADCs are 
controlled. Note, within Figure 3.13, the I2C buses are separated into their respective parts of 'bit bang' lines and isolated I2C. The internal components communicate with the 'bit bang' I2C, and the STF-1 flight computer communicates with the MCU via the isolated I2C. The various components and subsystems of the I-V characterizer are shown. 


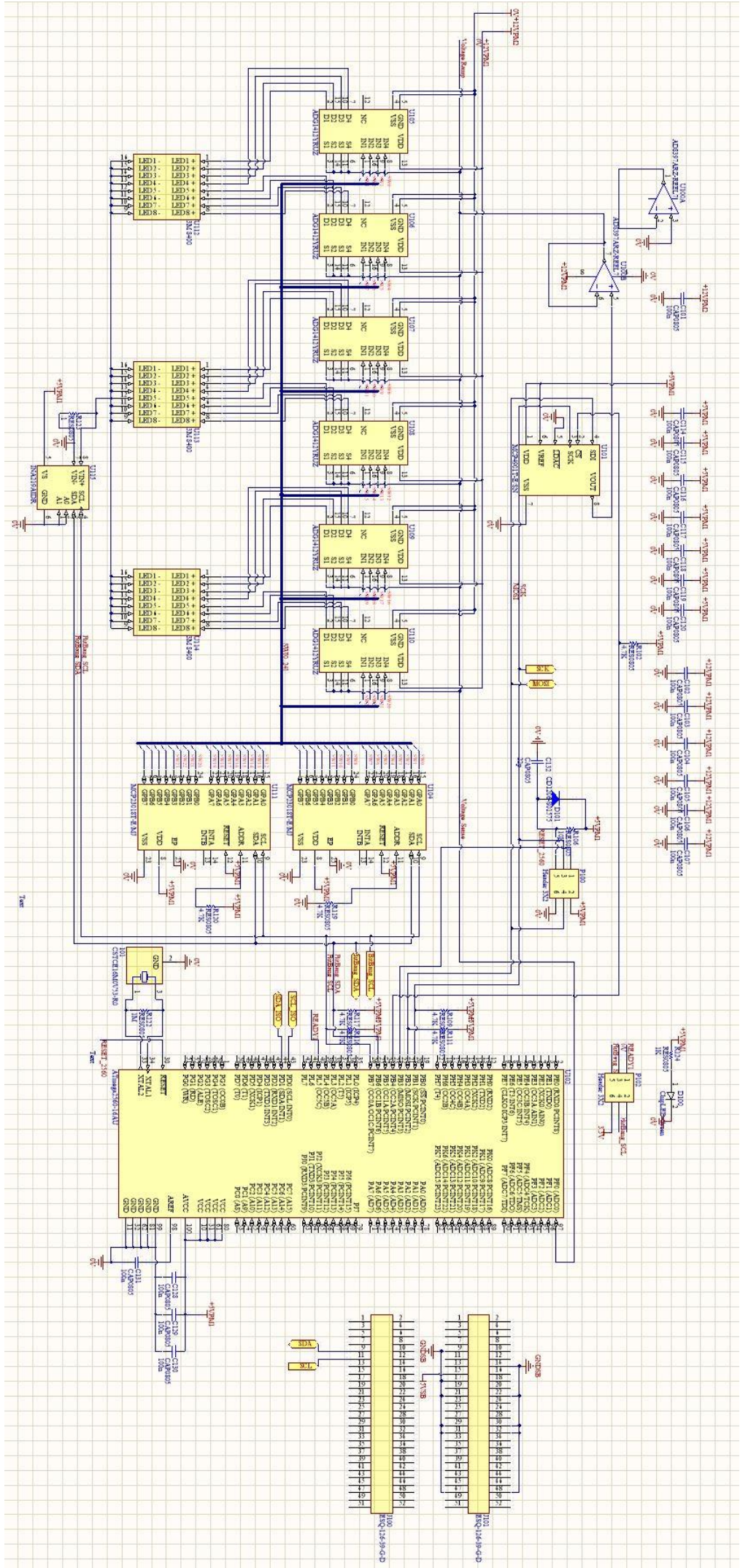

Figure 3. 12: Complete LOCC MCU/I-V Characterizer Circuit Diagram 


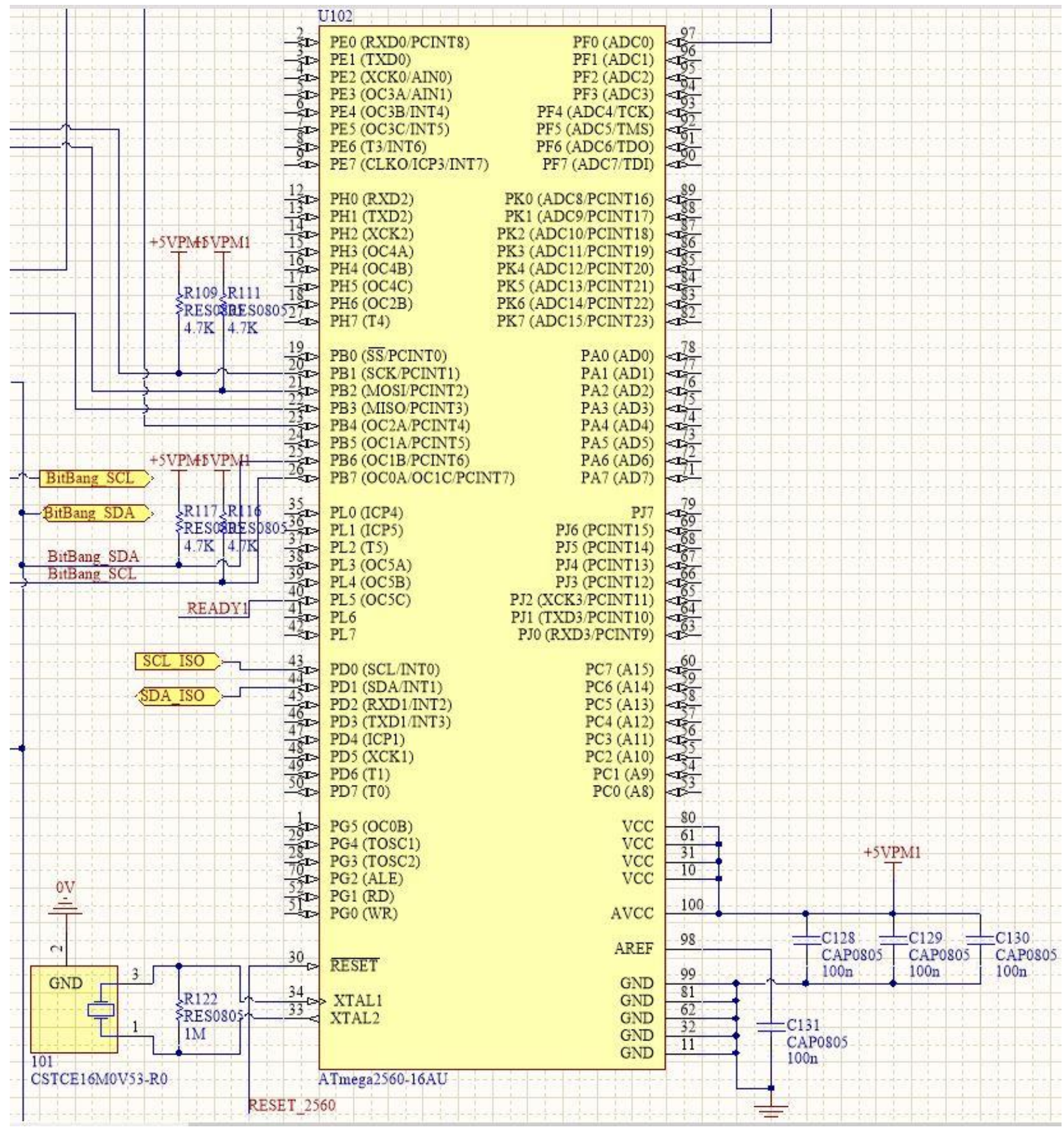

Figure 3. 13: LOCC MCU, the ATMEGA 2560-16AU. 
Figure 3.14 shows the LED driving circuit. A MCP4901 with SPI connection is used to control the applied voltage over the LEDs [157]. Note the chip select (CS) pin connected to the MCU. The CS pin his held low (0V). When this happens the MCP4901 does not "listen" or "see" the incoming data that is being sent via the Master Out Slave In (MOSI) line. This allows for multiple devices to communicate over the same bus. When active, the MCP4901 receives command bytes that set the output voltage from 0 to VREF. It is important to note that VOUT of the DAC can come within .02 $\mathrm{V}$ of the reference voltage, meaning the maximum output of VOUT to be around 4.98V. The DAC used provides for a voltage step size of .02V. The ideal voltage range is given as 0V to 255/256*VREF. Equation 3.2 shows the DAC analog output voltage calculation,

$$
V_{\text {out }}=\frac{V_{r e f} \times D_{n}}{2^{n}} G
$$

where VREF is the voltage reference, $\mathrm{Dn}$ is $\mathrm{DAC}$ input code, $\mathrm{G}$ is gain selection and $\mathrm{n}$ is the DAC resolution.

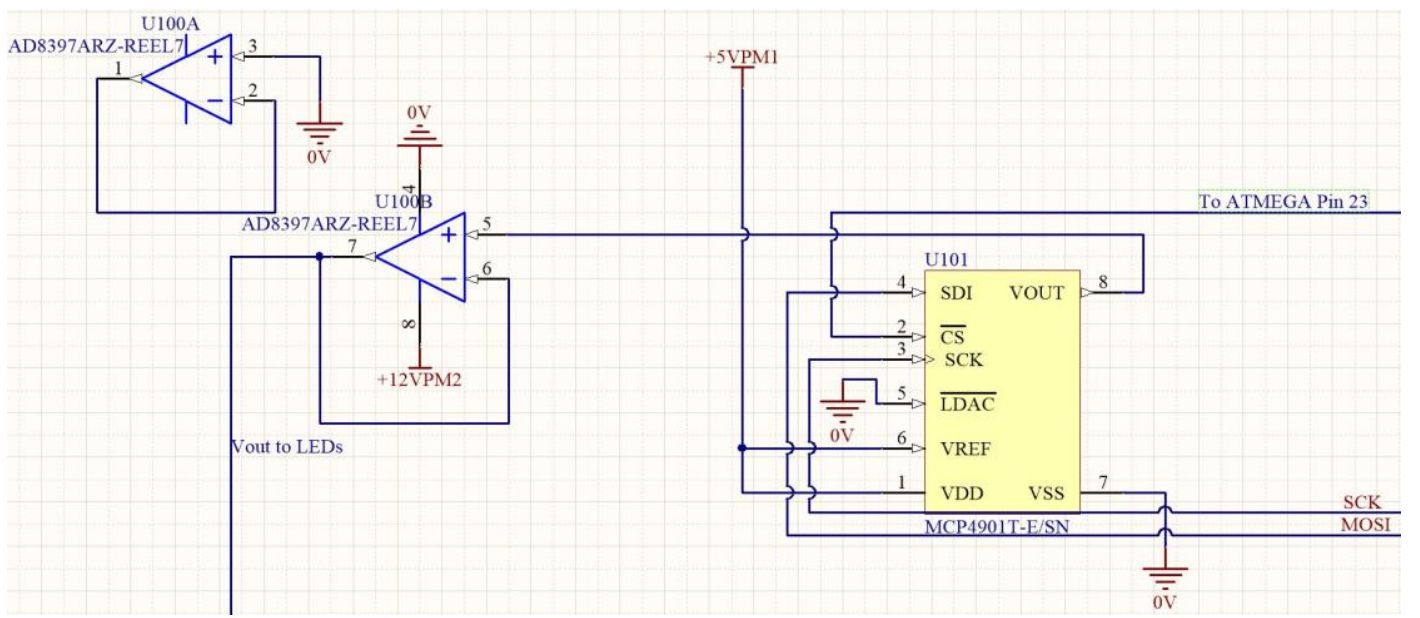

Figure 3. 14:Digital-to-Analog Converter connected to unity-gain OPAMP to source current to LEDs. 
The DAC is connected to the non-inverting terminal and configured for unity gain. With a unity gain configuration, the output of the opamp (VOUT) is given as:

$$
V_{\text {out }}=V_{\text {in }}
$$

where Vout is the opamp voltage output and Vin is the voltage output from the DAC. This allows for the direct translation of ramped voltage and the necessary supply of current for turning on LEDs.

The opamp selected is the Analog AD8397 Rail-to-Rail, high output current amplifier [15]. To ensure proper voltage swing, the rails of the opamp are connected to ground and the $12 \mathrm{~V}$ plane. This opamps provides a linear response to driving heavier loads, such as a LED, with up to $310 \mathrm{~mA}$ outputs. Note that in Figure 3.14 there are two opamps shown. The second opamp is grounded so that it does not interfere with the one supplying the current to the LEDs. Figure 3.14 describes the sourcing method for driving the LEDs and supplying the needed amount of current.

Figure 3.15 describes the circuits and components used to switch the LEDs, their connections, and the current sensing IC. From the sourcing circuit, the VOUT of the opamp is connected to the ADG1412 source pins. The ADG1412 is from Analog Devices, and is a quad single-pole/single-throw (SPST) switching IC with low 'on' resistance [159]. The switch uses metal-oxide semiconductor (CMOS) technology to control which LED turns on. The ADG1412 allows for a maximum of 190 $\mathrm{mA}$ for the TSSOP package, which is adequate current for the LEDs. The ADG1412 is also controlled via logic levels. These logic levels are controlled by a set of general-purpose input/output (GPIO) expander ICs. The expander is provided by Microchip Technology and is the MCP23018 16-bit I/O Expander with Open-Drain Outputs [160]. 


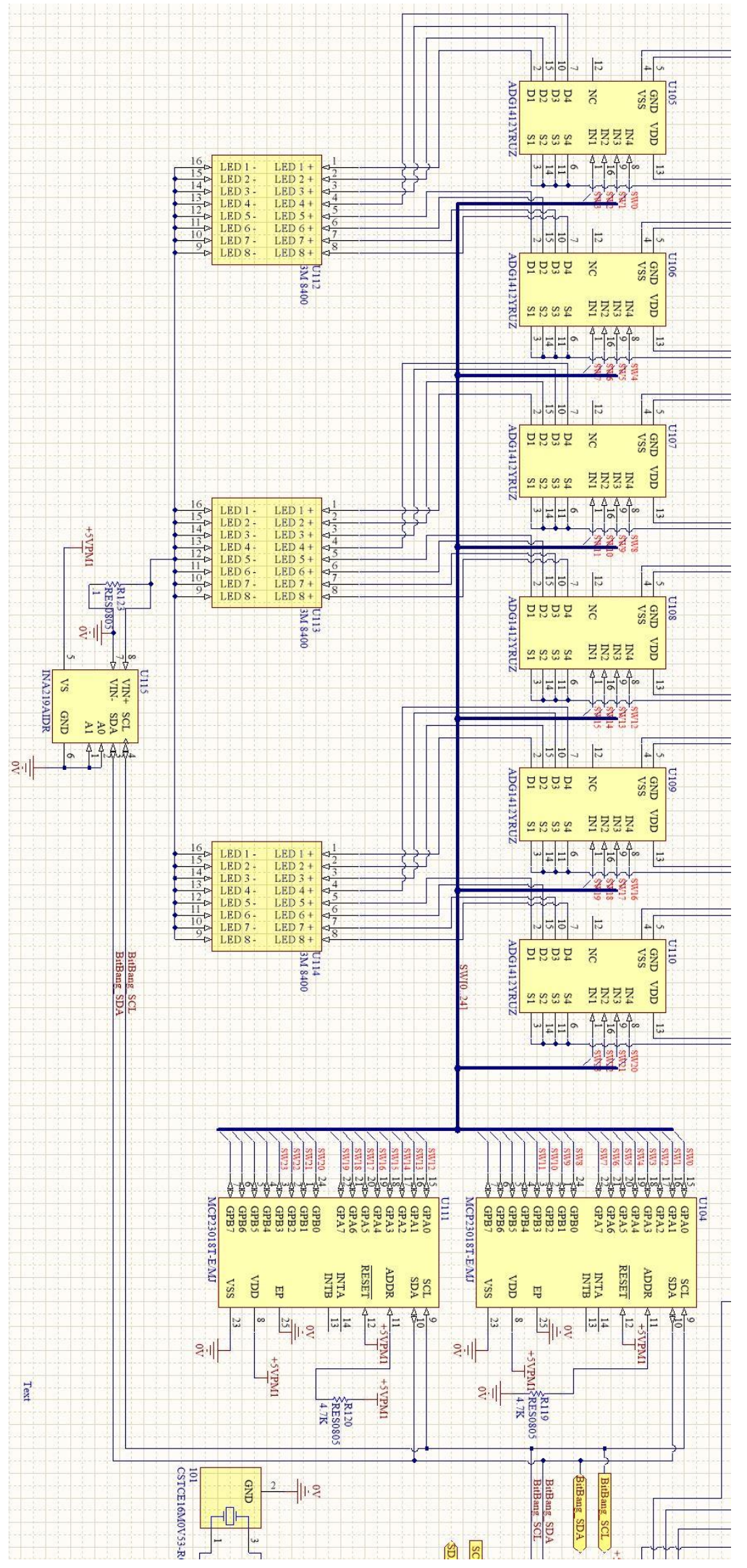

Figure 3. 15: LOCC LED Control Circuit 
The MCP23018 16-bit GPIO expanders are controlled via $100 \mathrm{KHz}$ I2C interfacing. The devices are highly configurable in that they utilize registers for determining input, output and polarity selection. The MCU can configure the MCP23018 for the I/O pins to be either input or output. These I/O pins also come equipped with built in pull-up resistors, so the addition of discrete resistors is not necessary to supply valid logic levels. This makes the MCP23018 usable for control of the ADG1412 for automated switching control.

From the control of the GPIO expanders for the SPST switches, the current can now be directed to the LED of choice in the chip carrier socket, listed as components U112 to U114. The output of each of these chip carrier sockets is then connected to a common node at resistor R123. Figure 3.16 describes the configuration of the current sensor connected to the common LED output node.

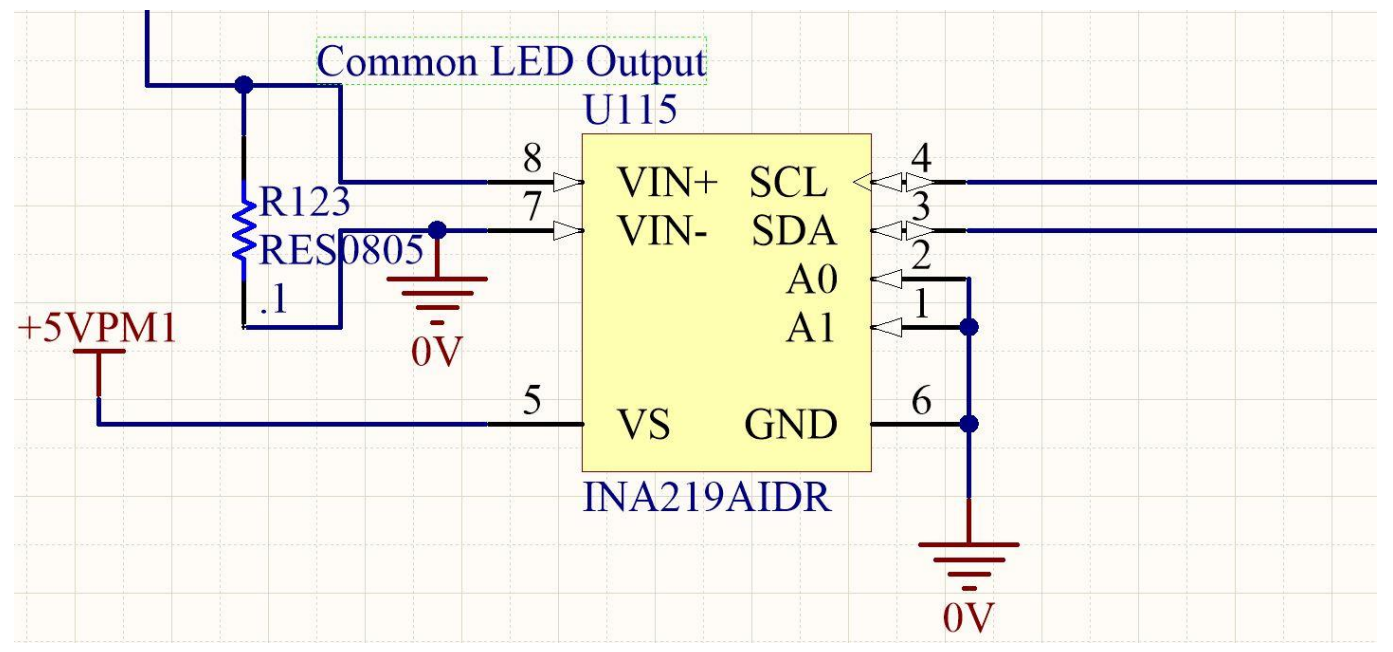

Figure 3. 16: LOCC Current/Power Monitor Circuit. 
Current and power monitoring are performed using the INA219 Zero-drift, Bi-directional Current/Power Monitor [161] from Texas Instruments. The INA219 is configured with a $0.1 \Omega$ shunt resistor for high-side current monitoring. The device communicates via I2C for monitoring of shunt voltage drop. The INA219 is also designed for temperature swings of $-40^{\circ} \mathrm{C}$ to $+125^{\circ} \mathrm{C}$, making it a reliable device for operations in space. This device is the final component in the operation of the I-V characterizer of the LOCC system. Figure 3.17 describes a sourcing configuration of the characterizer.

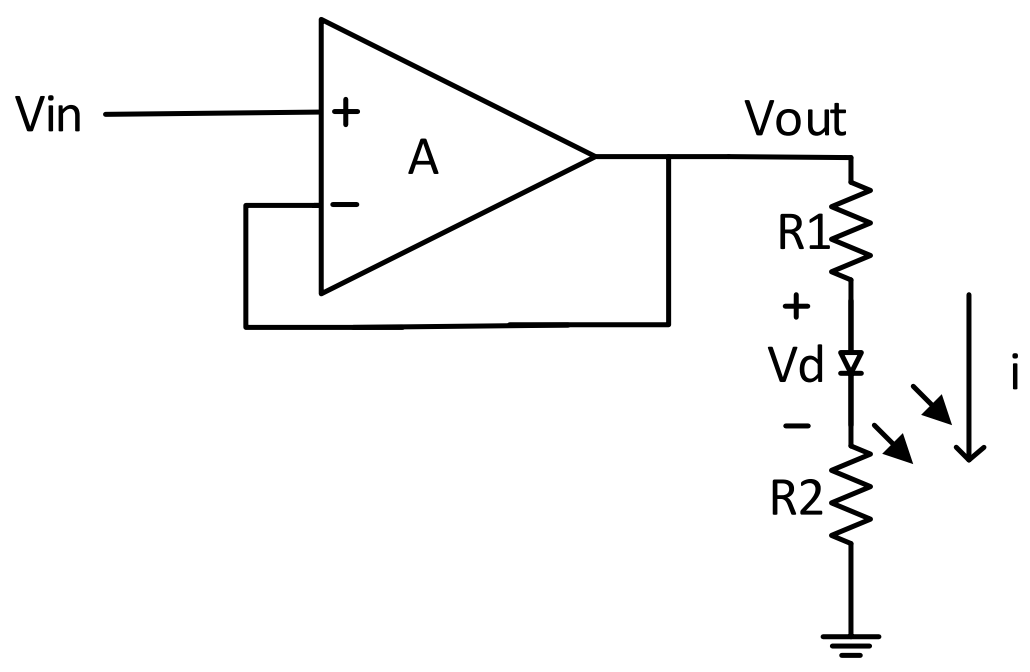

Figure 3. 17: Simplified LED Sourcing Circuit

Here, Vout is governed by the MCP4901, R1 is the resistance of the SPST switch, R2 is the Resistance of the shunt resistor, $\mathrm{i}$ is the current, and $\mathrm{Vd}$ is the turn-on voltage of the LED. From here, the maximum amount of current that can flow through the circuit can be determined. The maximum current flowing through the LED should be limited to protect the opamp and current sensor. Current traveling through the circuit is governed by the Equation $\mathrm{XX}$,

$$
i=\frac{V_{\text {out }}-V_{d}}{R_{1}+R_{2}},
$$


where $V_{\text {out }}$ is the voltage from op-amp output, $V_{d}$ is the voltage drop across the LED, and $R_{1}$ and $R_{2}$ are the series resistances of the SPST switch and shunt resistor, respectively.

\subsubsection{Electroluminescent Module}

The LOCC system is comprised of both an I-V characterizer and a EL characterizer. This subsection will cover the EL characterizer circuit and key components used in the creation of this board. Figure 3.18 shows the circuit diagram of the EL characterizer. 


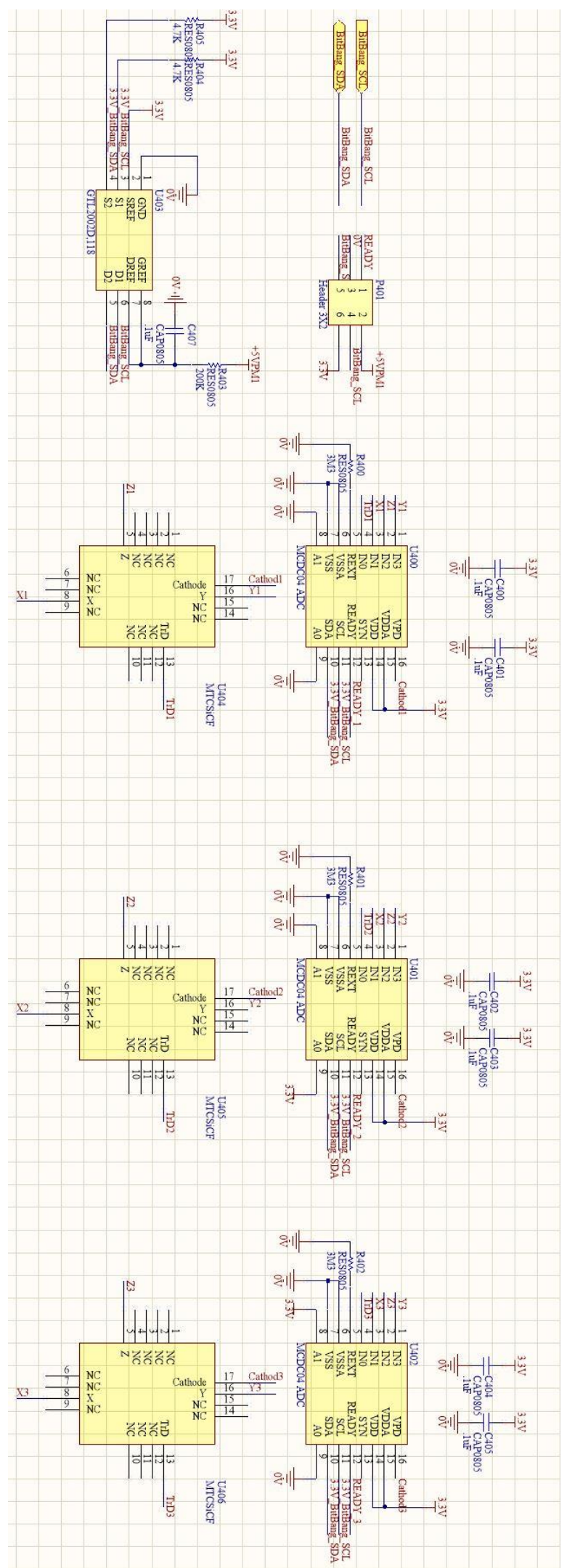

Figure 3. 18: EL Characterizer Complete Circuit 
Initial testing was performed using the MAZe'T passive OEM sensor board to evaluate the CIE1931 color standard [163]. This board allowed the development of the firmware and proper selection of the hardware components for use in the LOCC EL characterizer. The EL characterizer is comprised of an ADC, the MAZe'T MCDC04 and connected to a Si-PIN photodiode array, the MTCSiCF XYZ color sensor [163]. The MCDC04 and the MTCSiCF color sensors are repeated three times to align with each set of LEDs. Figure 3.19 shows the single system circuit diagram.

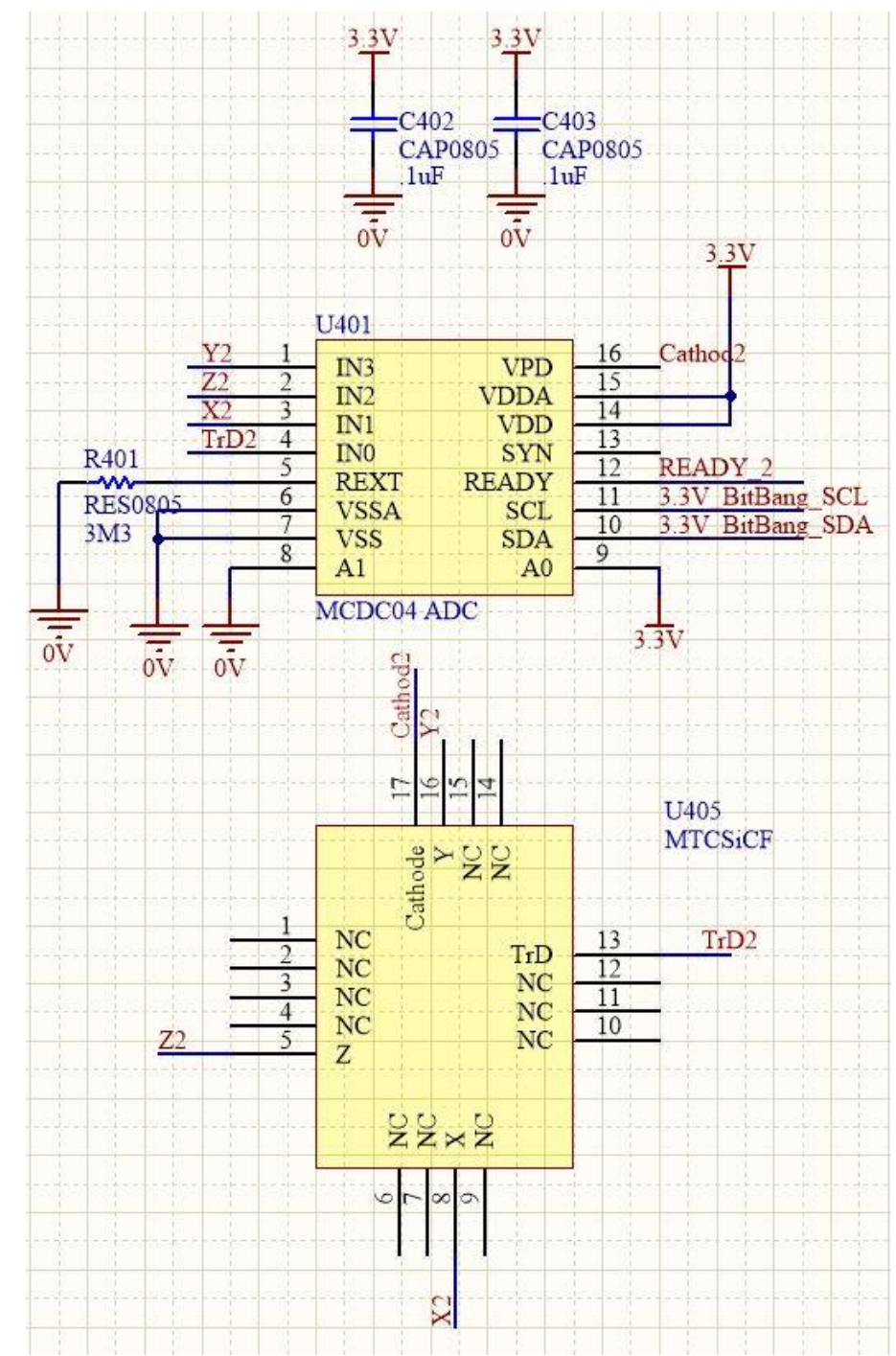

Figure 3. 19: Single ADC-to-Photodiode Circuit 
The labels can be seen for the interconnection between the components. The MCDC04 performs current to digital conversion by utilizing four parallel connected ADCs. This device sets the sensitivity, start and stop conversions for applications of interest. Note the $3.3 \mathrm{M} \Omega$ resistor. The external resistor REXT directly influences the generating of the reference current for the signal conversion. Therefore, the temperature coefficient of resistance has an important role. The smaller temperature coefficient and the resistor value tolerance $(1 \%$ better than $5 \%$, etc.) result in more accurate conversion.

For the detection of color, the MTCSiCF True Color Sensor IC provides a filter function based on CIE 1931/DIN 5033 color standards, which is based on the human eye. This component provides long-term stability over time and temperature. A single sensor consists of 19x3 photodiodes that have been integrated into a single package of a hexagonal matrix with $2 \mathrm{~mm}$ diameter. The device has been used in general purpose applications of color measurement and control, portable color measurements, and detection of light sources and regulated color temperatures [163]. The packaging of the sensor IC has an aperture angle (beam width) of nearly $90^{\circ}$. Traditional interference filters operate depending on angle of incidence. Using a $<10^{\circ}$ angle of incidence will allow the best results with no filter shifts. This can be ensured by using lenses or optical holes that limit the angle of incidence to the sensor device $<10^{\circ}$. An angle of incidence of more than $10^{\circ}$ will result in a filter shift. The filter response and accuracy will be distorted the greater the angle deviation is. LOCC operates within this constraint by having the photosensors directly above the LED chip carriers. Using the dimensions provided in figure 3.7, the angle of incidence is approximately $6^{\circ}$. This falls within the constraints to avoid filter shifting.

It is important to note the $3.3 \mathrm{~V}$ I2C and voltage supplies. The LOCC system provides a stepped-down voltage to the EL characterizer for the operation of the ADCs. The I2C logic level must also match the voltage supply. The logic translation is done using NXP's GTL2002 2-bit bidirectional low voltage translator [171]. Figure 3.20 shows the circuit diagram of the translator circuit 
for the EL characterizer. The Gunning Transceiver Logic (GTL) IC provides high-speed voltage translation for a $5 \mathrm{~V} \mathrm{I} 2 \mathrm{C}$ logic to a $3.3 \mathrm{~V}$ I2C voltage level. This device provides for a bidirectional voltage translation between $1 \mathrm{~V}$ and $5 \mathrm{~V}$ without directional control.

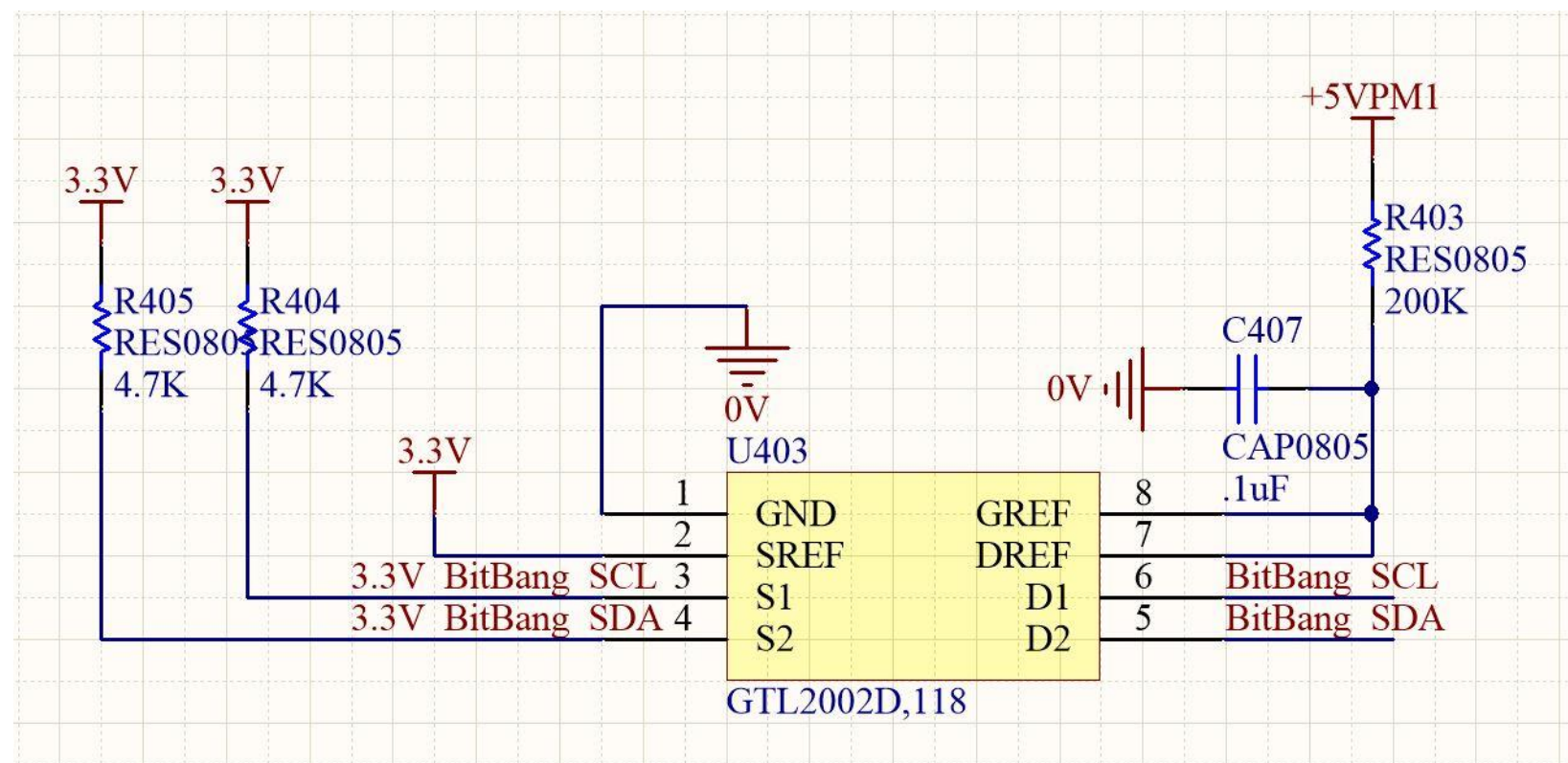

Figure 3. 20: Logic Translation circuit of EL Characterizer

\subsubsection{I2C Isolation Module}

Because the I2C bus of STF-1 experiences loading effects of connected communication circuits, LOCC provides an internal I2C isolation module to avoid single ground loops. The ground loop can be detrimental due to the grounded loop currents deteriorating the clock and data signals, and they can even damage circuit components. The I2C isolation module allows for communication between the LOCC MCU and STF-1's flight computer for telemetry packet delivery and receiving commands codes from the flight computer. This is done using Texas Instrument's ISO1540 LowPower Bidirectional I2C Isolator [168]. The ISO1540 comes with two bidirectional channels for clock and data, making it perfect for master and slave communications. The circuit diagram for the I2C isolation module is given in Figure 3.21. 


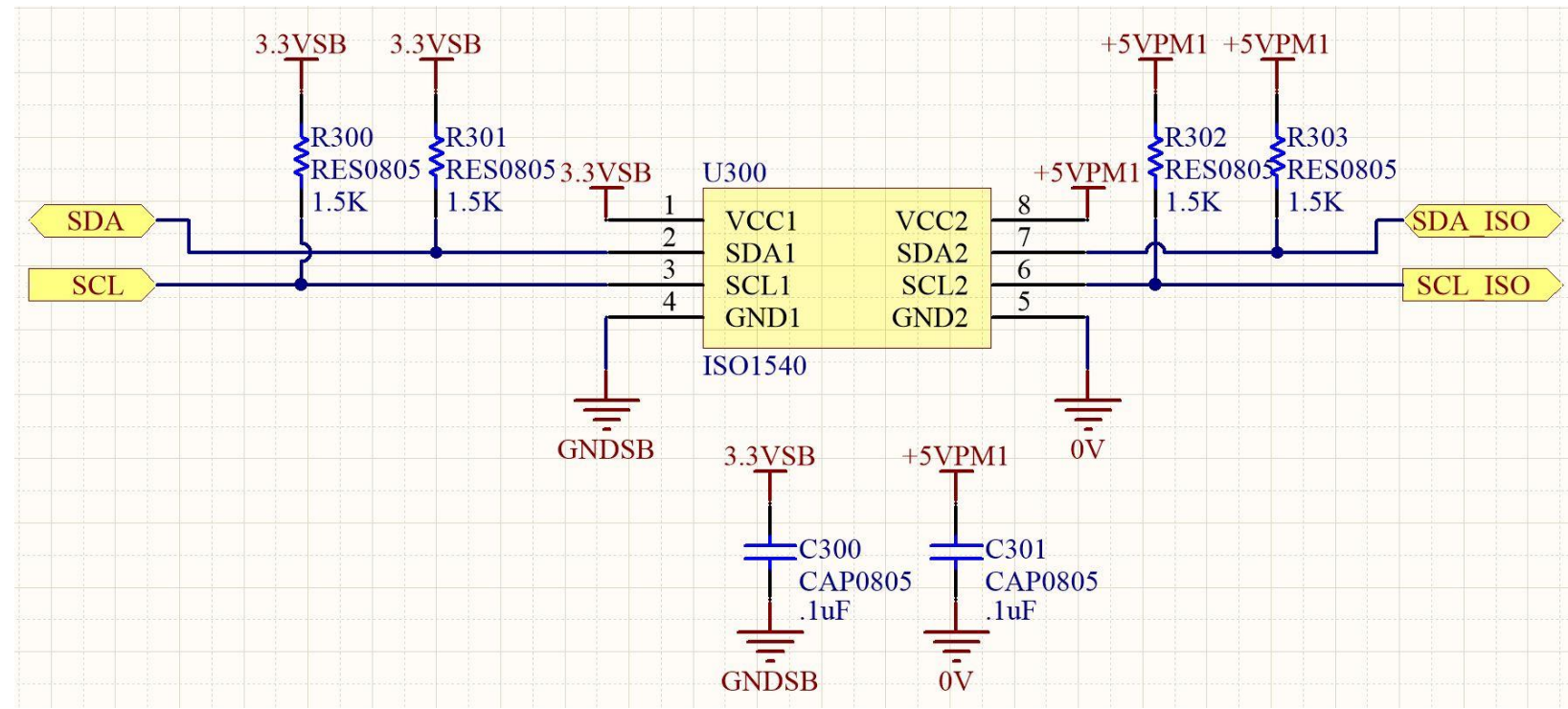

Figure 3. 21: LOCC I2C Isolation Module Circuit Diagram

\subsubsection{Power Supply Module}

The last module of the LOCC system is the power management module. The voltage supplied from the electronic power supply (EPS) of STF-1 is connected to the components of the LOCC system. Isolation is also employed to ensure safety between LOCC and the rest of STF-1's 5V power bus. This is done because the loading effects of the supplied power are unpredictable, and limiting the effects of outside nominal operation protects the components. This also provides a common ground for all components to operate from. Power management is done using CUI INC's PDS2-M DC-DC converter [167]. This IC comes in multiple packages which include $5 \mathrm{~V}$ to $24 \mathrm{~V}$ packages for voltage translations. For LOCC, two DC-DC converters are used to supply isolated $5 \mathrm{~V}$ and isolated $12 \mathrm{~V}$. This is done using the PDS2-S5-S5-M model and the PDS2-S5-S12-M model. These devices provide 2W of isolated power, short circuit protection, $79 \%$ and $82 \%$ efficiencies, and operate within $-40^{\circ} \mathrm{C}$ to $105^{\circ} \mathrm{C}$. To provide $3.3 \mathrm{~V}$ for components, a low-dropout regulator is used. The MICREL MIC5225 provides $5 \mathrm{~V}$ to $3.3 \mathrm{~V}$ conversion with a guaranteed $150 \mathrm{~mA}$ output [168]. The regulator can provide excellent load regulations and current protection for devices. This device also operates within a wide 
temperature range of $-40^{\circ} \mathrm{C}$ to $125^{\circ} \mathrm{C}$, making it a perfect component for the LOCC system in voltage regulation.

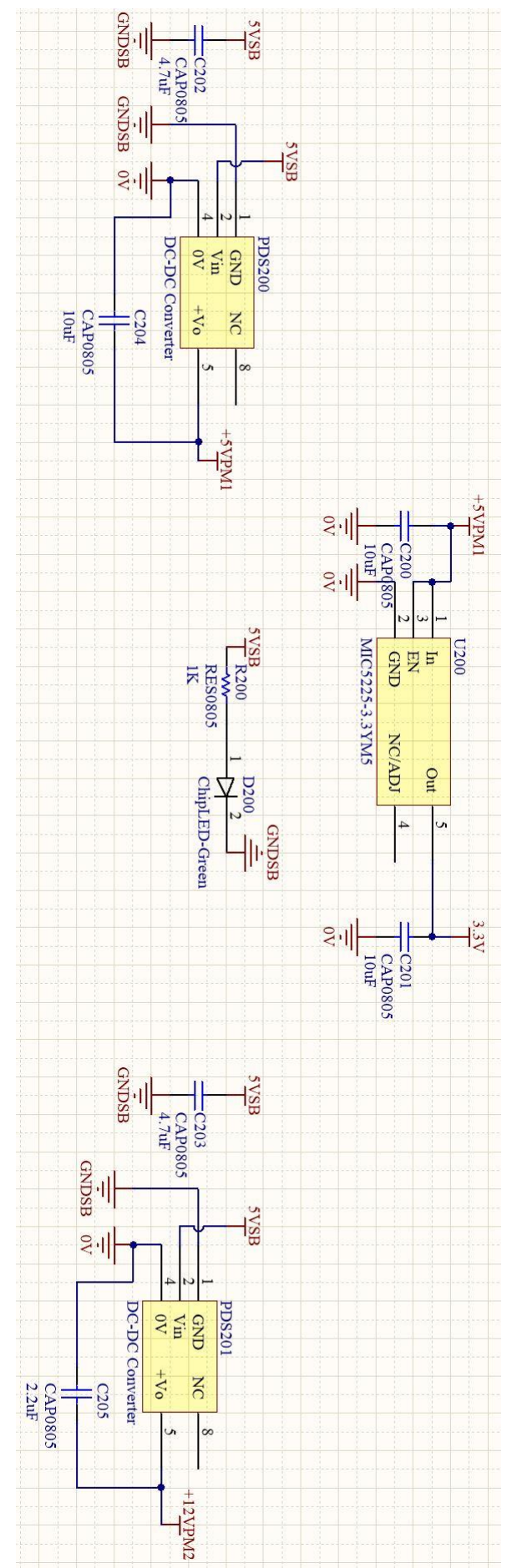

Figure 3. 22: LOCC Power Management Circuit Diagram 


\subsection{Printed Circuit Board Layout}

A printed circuit board (PCB) is designed using computer aided design (CAD) software that generates fabrication files known as Gerber files. These output files include copper patterns, solder masks images, drill files, and electrical test files. The fabricated boards go through multiple processing steps depending on the complexity of the board. This includes additive or subtractive processing, chemical etching, drilling, lamination, and plating [172].

PCBs are made to support electrical connections between components using conductive traces, and pads that can be etched from copper sheeting. Components such as capacitors, resistors and ICs are then soldered onto the PCB. The design of modern PCBs includes the following steps [172] [173]:

1. Schematic Capture

2. Board Dimensions

3. Components position determination

4. PCB layer stackup

5. Line impedance calculations

6. Vias and lands markings

7. Trace routing

8. Gerber File generation

This section will detail the design and layout of the LOCC system and the respective boards that make up the system. This includes detailing the board layers, signal routing, and board sizing. 


\subsubsection{I-V Characterizer PCB Layout}

The LOCC I-V characterizer consists of a 4-layer board with a finished thickness of 1.5748 $\mathrm{mm}(.062$ ") and a $2 \mathrm{oz}$. copper pour, or $0.203 \mathrm{~mm}$ thickness. For future designs, the copper pour thickness can be decreased to $1 \mathrm{oz}$. for a reduction in cost. Both LOCC boards follow PC104 standard and the dimensions can be found in Figure 3.23. Table 3.3 describes the board layers and the final height/thickness of the entire board.

Table 3. 3: I-V Characterizer Board Stack Report

\begin{tabular}{|c|c|c|c|c|}
\hline \multicolumn{5}{|c|}{ Board Stack Report } \\
\hline \multicolumn{2}{|r|}{ Stack Up } & \multicolumn{3}{|c|}{ Layer Stack } \\
\hline Layer & Board Layer Stack & Name & Material & Constant \\
\hline 1 & & Top Paste & & \\
\hline 2 & & Top Overlay & & \\
\hline 3 & & Top Solder & $\begin{array}{l}\text { Solder } \\
\text { Resist }\end{array}$ & 3.5 \\
\hline 4 & & Top Side & Copper & \\
\hline 5 & & Dielectric 1 & FR-4 & 4.2 \\
\hline 6 & $\square$ & $\begin{array}{l}\text { Ground } \\
\text { Plane }\end{array}$ & Copper & \\
\hline 7 & & Dielectric 3 & & 4.2 \\
\hline 8 & & Power Plane & Copper & \\
\hline 9 & & Dielectric 4 & & 4.2 \\
\hline 10 & & Bottom Side & Copper & \\
\hline 11 & $\square \square \square \square$ & $\begin{array}{l}\text { Bottom } \\
\text { Solder }\end{array}$ & $\begin{array}{l}\text { Solder } \\
\text { Resist }\end{array}$ & 3.5 \\
\hline 12 & & $\begin{array}{l}\text { Bottom } \\
\text { Overlay }\end{array}$ & & \\
\hline 13 & & $\begin{array}{l}\text { Bottom } \\
\text { Paste }\end{array}$ & & \\
\hline & $\begin{array}{r}\text { Height: } 1.5748 \mathrm{~mm} \\
\left(.062^{\prime \prime}\right)\end{array}$ & & & \\
\hline
\end{tabular}




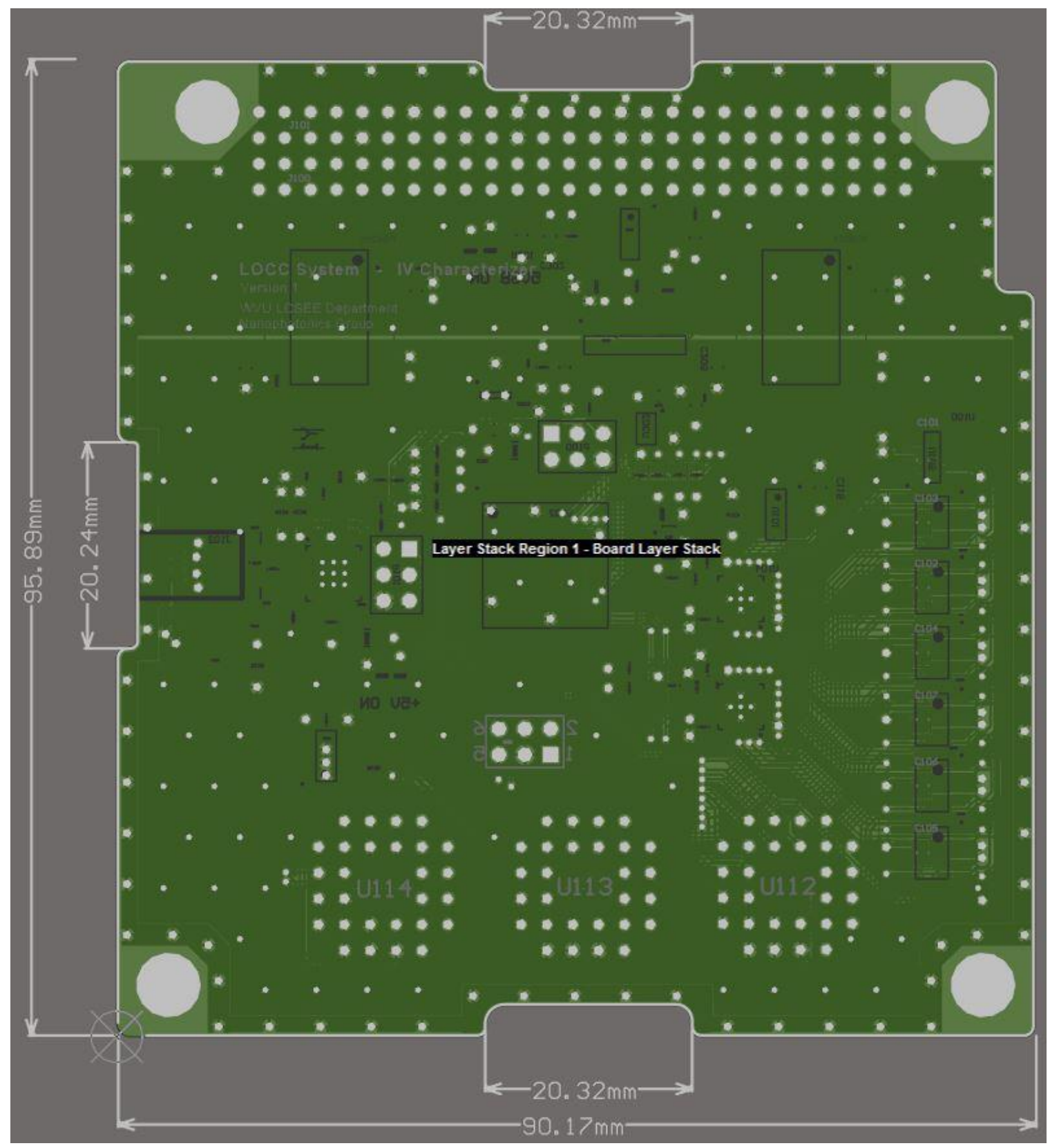

Figure 3. 23: Board Outline and dimensions of LOCC I-V Characterizer

Starting from the internal layers, the ground and power planes are located on the internal copper layers. The ground plane layout is shown in Figure 3.24. The visible green line denotes the outline of the ground plane copper, and the line extending across the top portion denotes the split between the system ground and the isolated ground. 


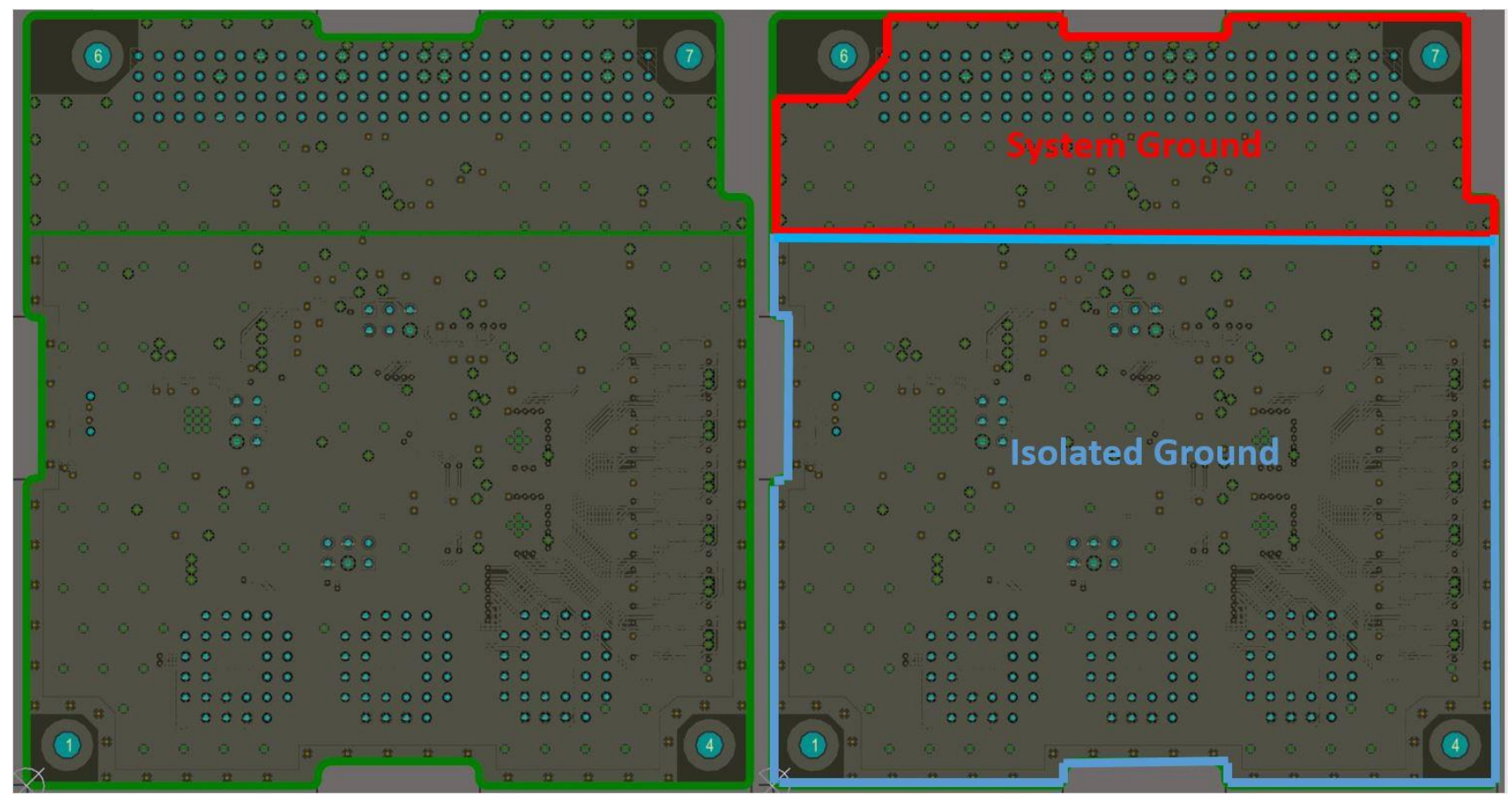

Figure 3. 24: (Left) Ground Plane layout, (Right) Designation between System Ground and Isolated Ground

Figure 3.24 illustrates a side-by-side comparison of ground plane layout and the separation of system ground and isolated ground planes. Both ground sections exist on the same plane, but are separated by a $0.533 \mathrm{~mm}$ pullback. The ground plane isolations are fundamental to the operation of LOCC and are used with the DC-DC converters. This design consideration helps prevent damages that may be caused by fluctuations in system power and avoid ground loops, keeping digital signals clean from power anomalies.

Like the ground plane, the power plane is split between the system bus power and the isolated plane power. While the ground plane provides an isolated ground for all components, the isolated power supply is split into a $5 \mathrm{~V}$ isolation and a $12 \mathrm{~V}$ isolation to power the switches and the opamp. Figure 3.25 describes the power plane layout for the separation of the system power plane and the isolated $5 \mathrm{~V}$ and $12 \mathrm{~V}$ planes. 


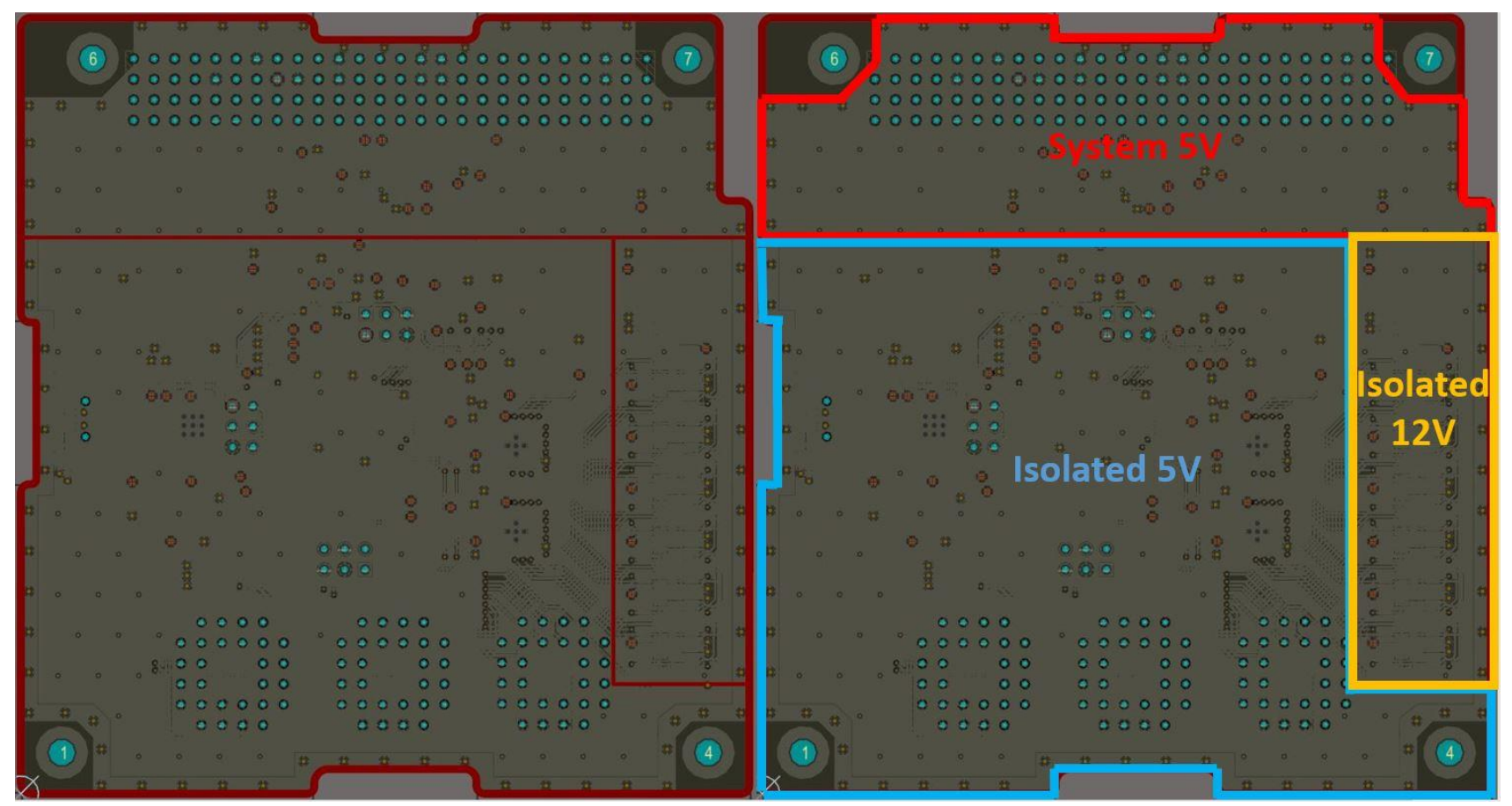

Figure 3. 25: (Left) Power Plane Layout, (Right) Designation between System 5V Power, Isolated 5V, and Isolated $12 \mathrm{~V}$

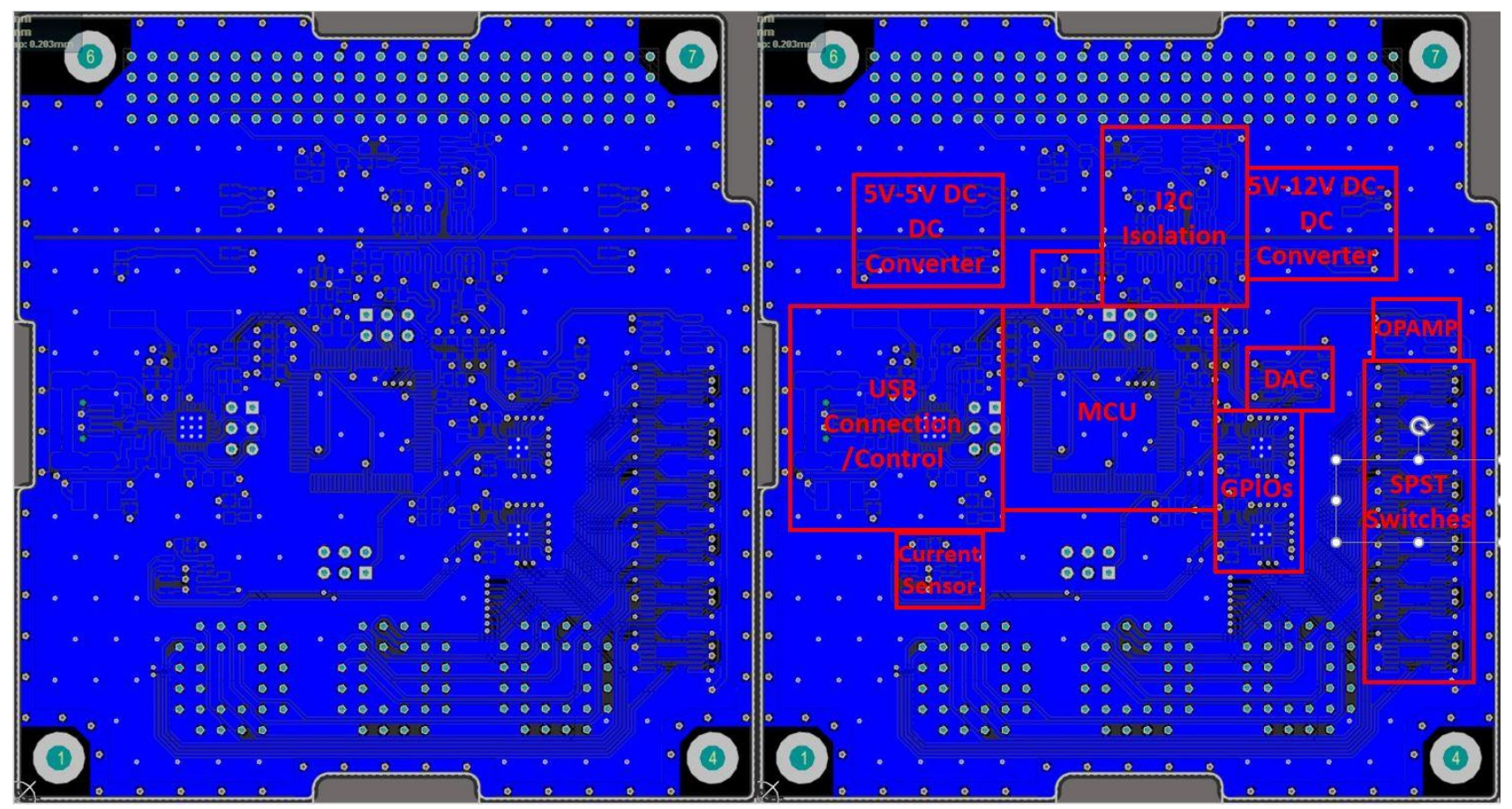

Figure 3. 26: (LEFT) Bottom Signal Plane Layout, (Right) Designation of modules and components 
Figure 3.26 shows the bottom layer layout of the PCB. This includes trace routes and the location of key components, as described by the schematics in Section 3.4. This gives an idea of where components are located for further diagnosis, and provides a mapping of traces for testing signal and power integrity. The top layer of the LOCC I-V system is given in Figure 3.27. There are few components placed on this side of the board. The LED chip carrier sockets are placed to face toward the photodiodes.

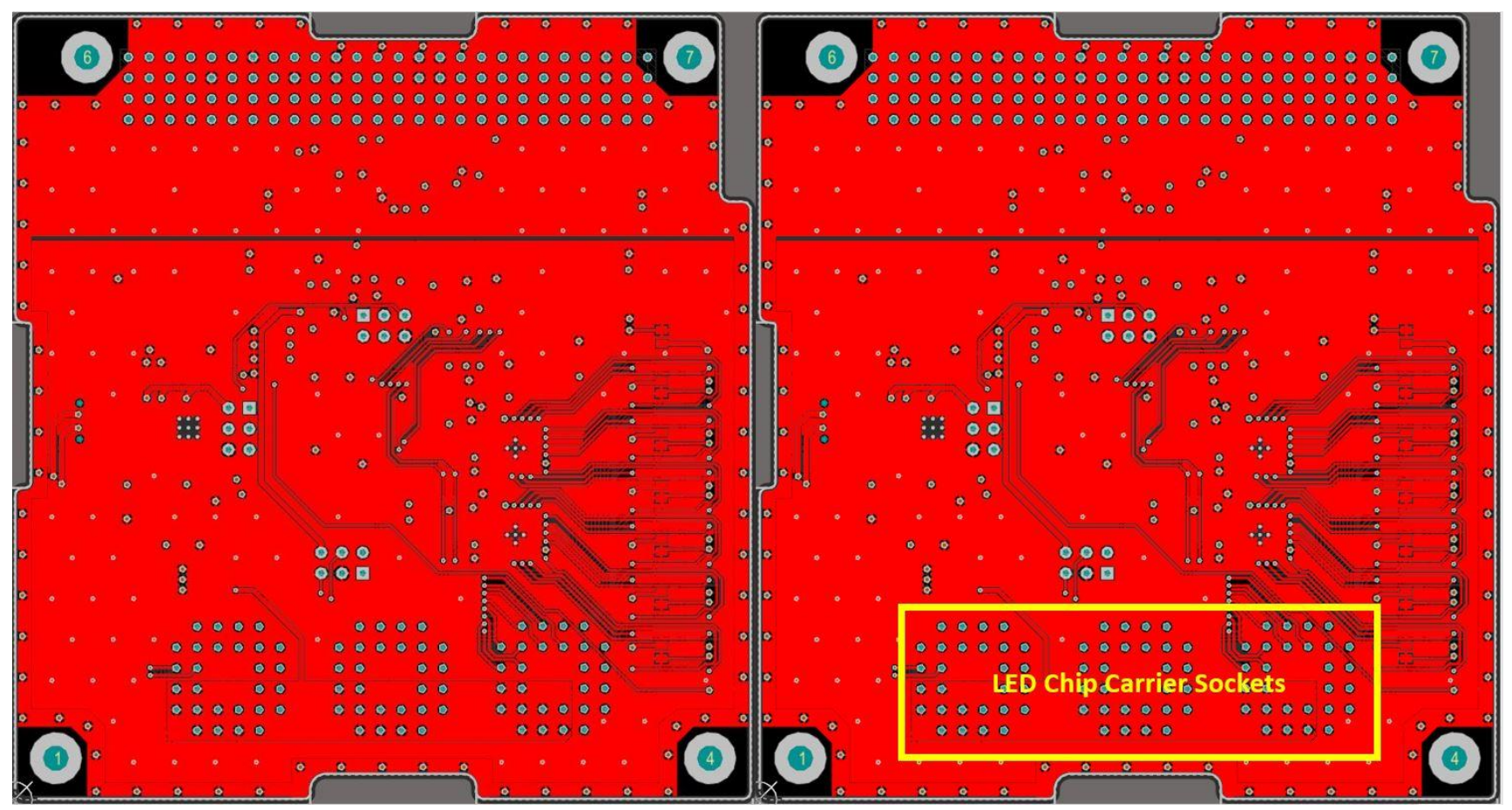

Figure 3. 27: (Left) Top Signal Plane Layout, (Right) Designation of chip carrier socket components

The last portion of images illustrate an isometric view of both the top and bottom planes of the LOCC system before fabrication. The 3D rendered view gives an idea of the space required by the components, and describes the placement of the components. Figure 3.28 shows the isometric 3D images of the LOCC I-V characterizer. 


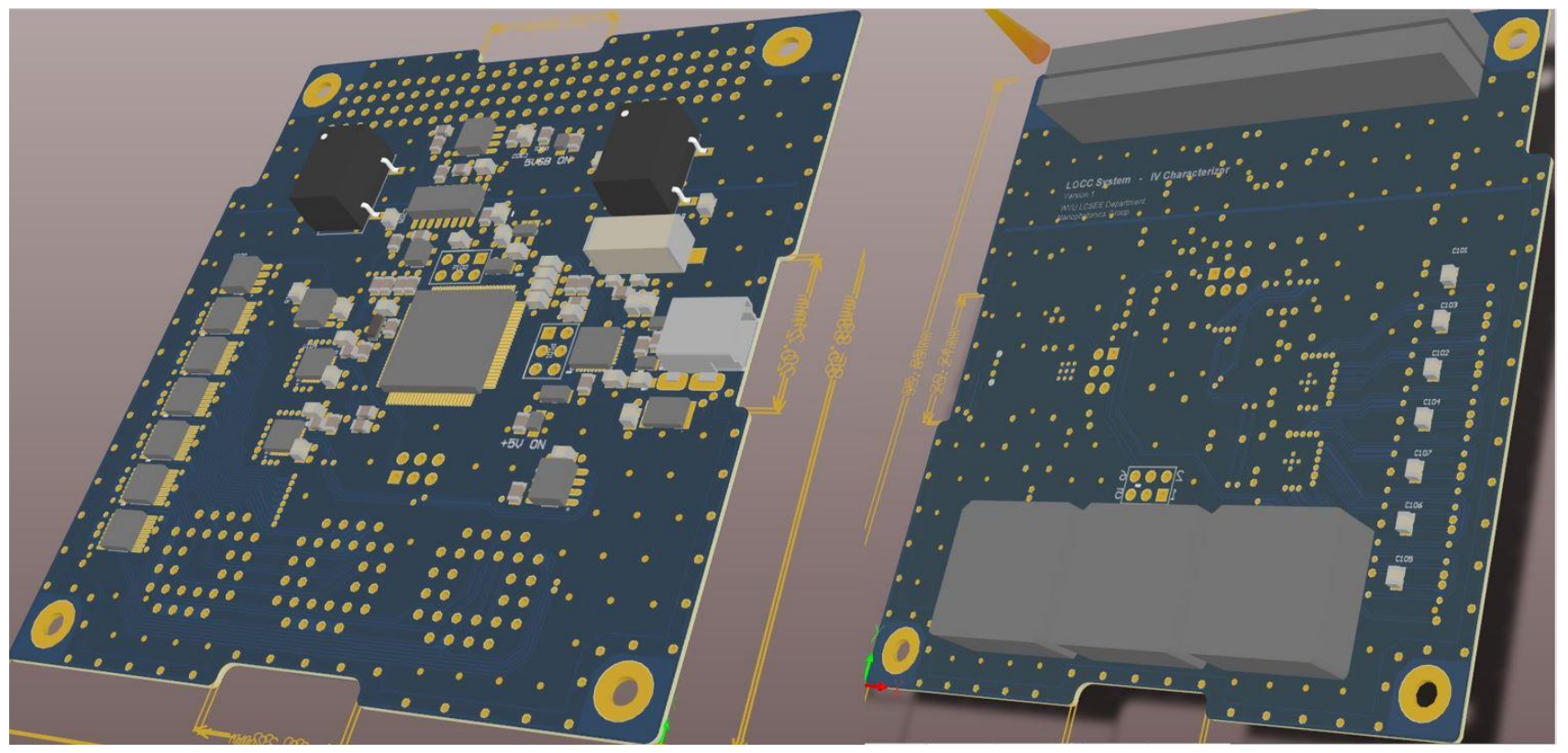

Figure 3. 28: (Left) 3D View Bottom Side, (Right) 3D View Top Side 


\subsubsection{EL Characterizer PCB Layout}

The LOCC EL characterizer consists of a 2-layer board with a finished thickness of 1.5748 $\mathrm{mm}(.062$ ”) and a 1 oz. copper pour, or $0.203 \mathrm{~mm}$ thickness. Table 3.4 describes the board layers and the final height/thickness of the entire board.

Table 3. 4: EL Characterizer Board Stack

\begin{tabular}{|c|c|c|c|c|}
\hline \multicolumn{5}{|c|}{ Board Stack Report } \\
\hline \multicolumn{2}{|r|}{ Stack Up } & \multicolumn{3}{|c|}{ Layer Stack } \\
\hline Layer & Board Layer Stack & Name & Material & Constant \\
\hline 1 & & Top Paste & & \\
\hline 2 & & Top Overlay & & \\
\hline 3 & & Top Solder & $\begin{array}{l}\text { Solder } \\
\text { Resist }\end{array}$ & 3.5 \\
\hline 4 & & $\begin{array}{l}\text { Component } \\
\text { Side }\end{array}$ & Copper & \\
\hline 5 & & Dielectric 1 & FR-4 & 4.2 \\
\hline 6 & & Bottom Layer & Copper & \\
\hline 7 & & Solder Side & $\begin{array}{l}\text { Solder } \\
\text { Resist }\end{array}$ & 3.5 \\
\hline 8 & 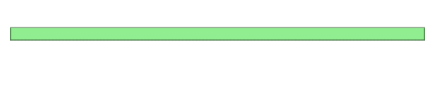 & $\begin{array}{l}\text { Bottom } \\
\text { Overlay }\end{array}$ & & \\
\hline 9 & & Bottom Paste & & \\
\hline & $\begin{array}{r}\text { Height: } 1.5748 \mathrm{~mm} \\
\left(.062^{\prime \prime)}\right.\end{array}$ & & & \\
\hline
\end{tabular}

The design of the LOCC EL characterizer is less complex than the I-V characterizer. The EL characterizer is made up of only two layers of copper as opposed to a 4-layer design. This helps reduce the overall cost of fabricating this portion of the system. Note that the final thickness of the board is still that of a 4-layer board. This is just a standard processing thickness from the PCB fabricators. The components are laid out to match the chip carrier sockets that are embedded on the other board. This 
allows for $<10^{\circ}$ viewing angle, ensuring no filter shifting from the photodiodes, as described in section 3.7. Figure 3.29 shows the board outline and planning for components and drills.

Because the board is a 2-layer board, the grounding and power supply planes are embedded on the surface. Like the ground planes of the I-V side, the bottom portion of the EL board is dedicated to components and a ground copper pour that is via-stitched to the opposite side. Figure 3.30 shows the placements of key components including the I2C voltage translator, ADCs and PDs. Turning to the top side, the $3.3 \mathrm{~V}$ volt plane is islanded within the isolated ground plane that is included on the top side. This allows for the components to be placed within the rectangular area for powering. Figure 3.33 shows the $3 \mathrm{D}$ rendering of the EL board to illustrate dimensions and concept.

Overall, the largest cost to this design are the components. The photodiodes and ADCs are highly sophisticated and are expensive. For future designs, more cost-effective components should be considered. It is recommended that newer designs not use the XYZ responsivity for determining peak wavelength emission. For reference, the company Pixelteq offers a multispectral sensor in 20 pin LCC packaging with 8 photodiodes tuned to specific wavelengths for the observation of peak wavelength emission [175]. Due to the required processing for a custom set of these Pixelteq photodiodes, lead times did not fall within the development schedule of the LOCC system. Given the necessary budget and scheduling these would make a significant addition to the LOCC EL characterizer. 


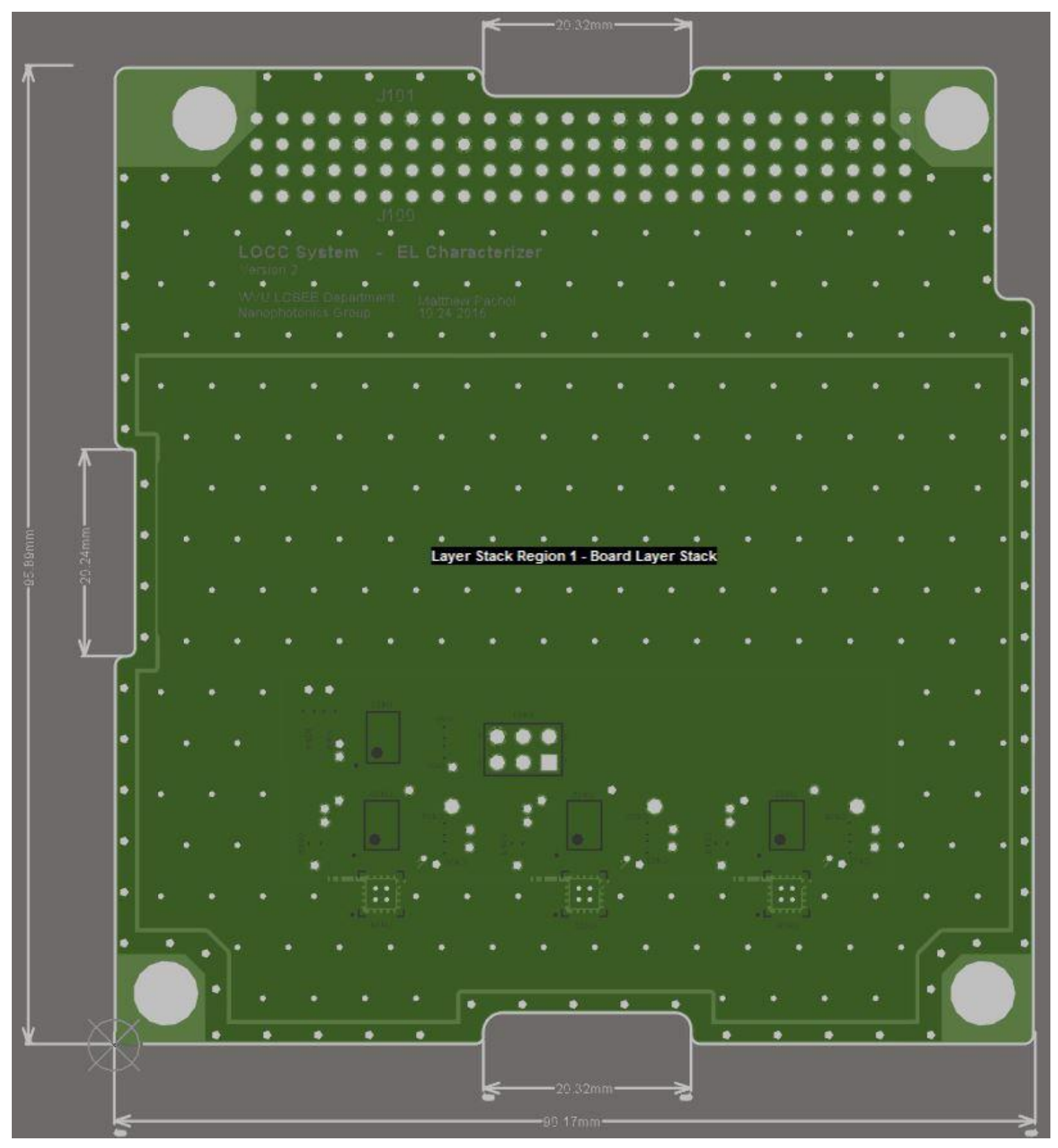

Figure 3. 29: Board Outline and Dimensions of LOCC EL Characterizer 


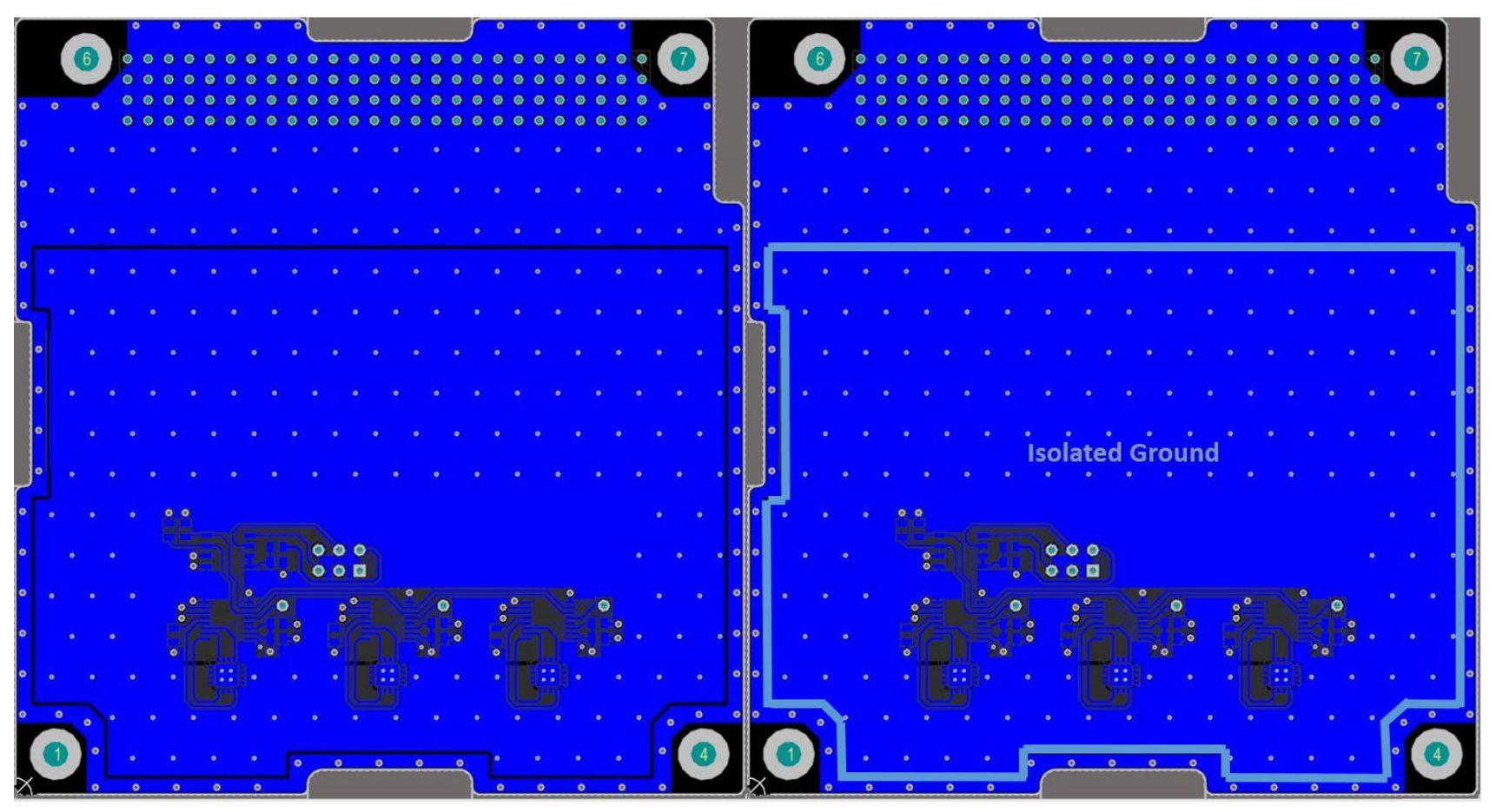

Figure 3. 30: LOCC EL Characterizer bottom side with outlined Isolated Ground Plane

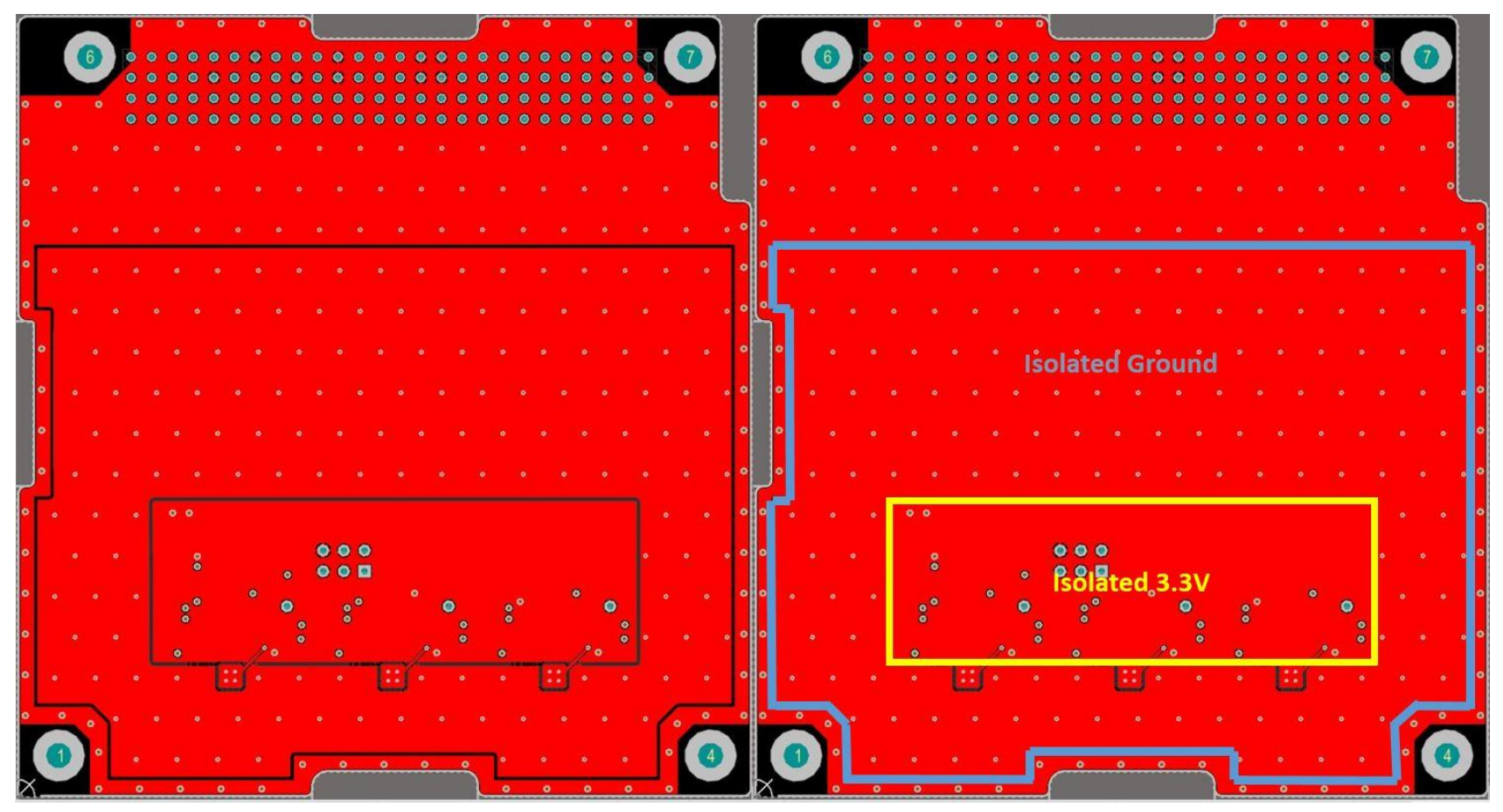

Figure 3. 31: LOCC EL Characterizer top side with outlined 3.3V and ground pours 


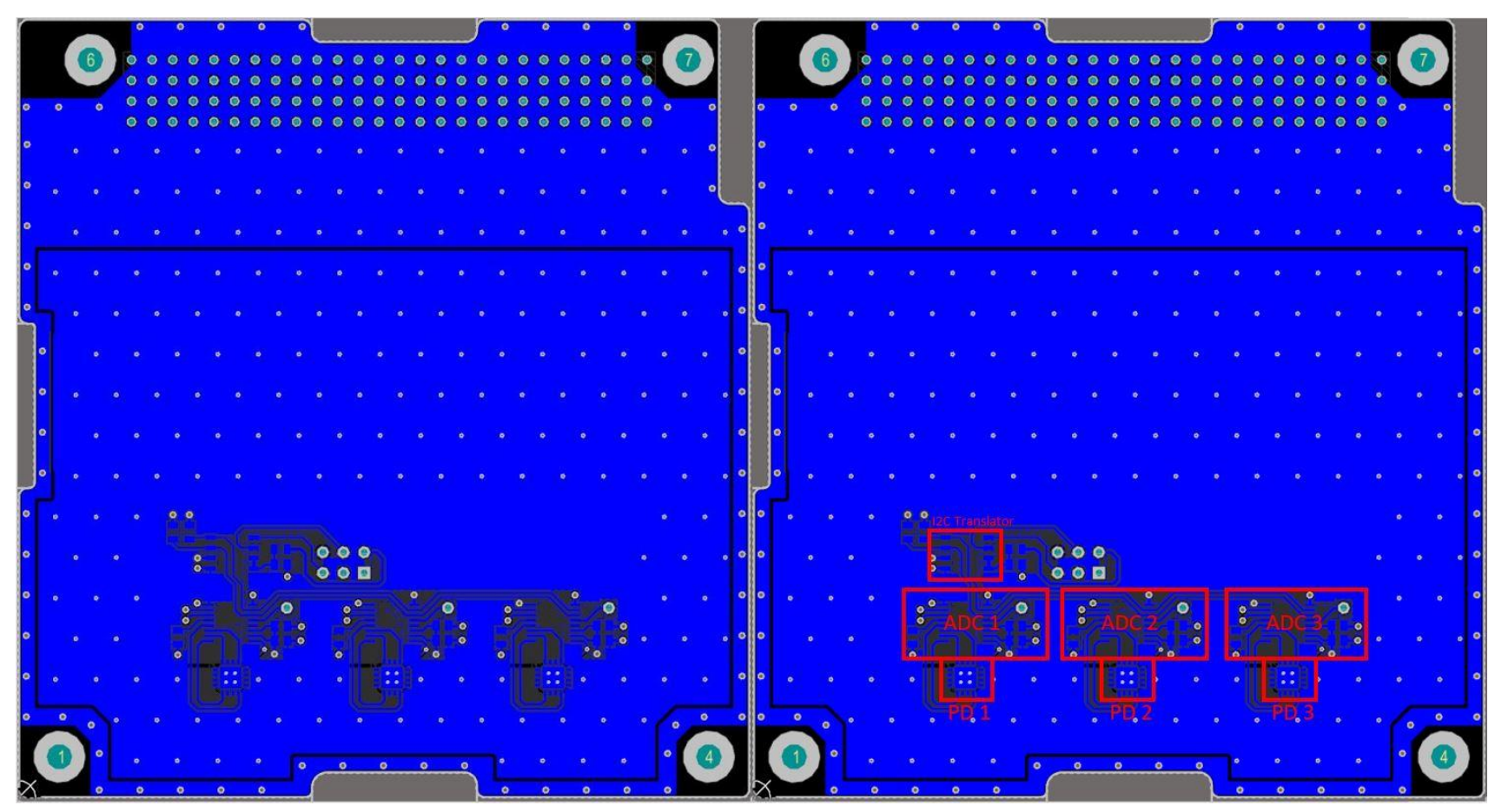

Figure 3. 32: (Left) Bottom Signal with designated components

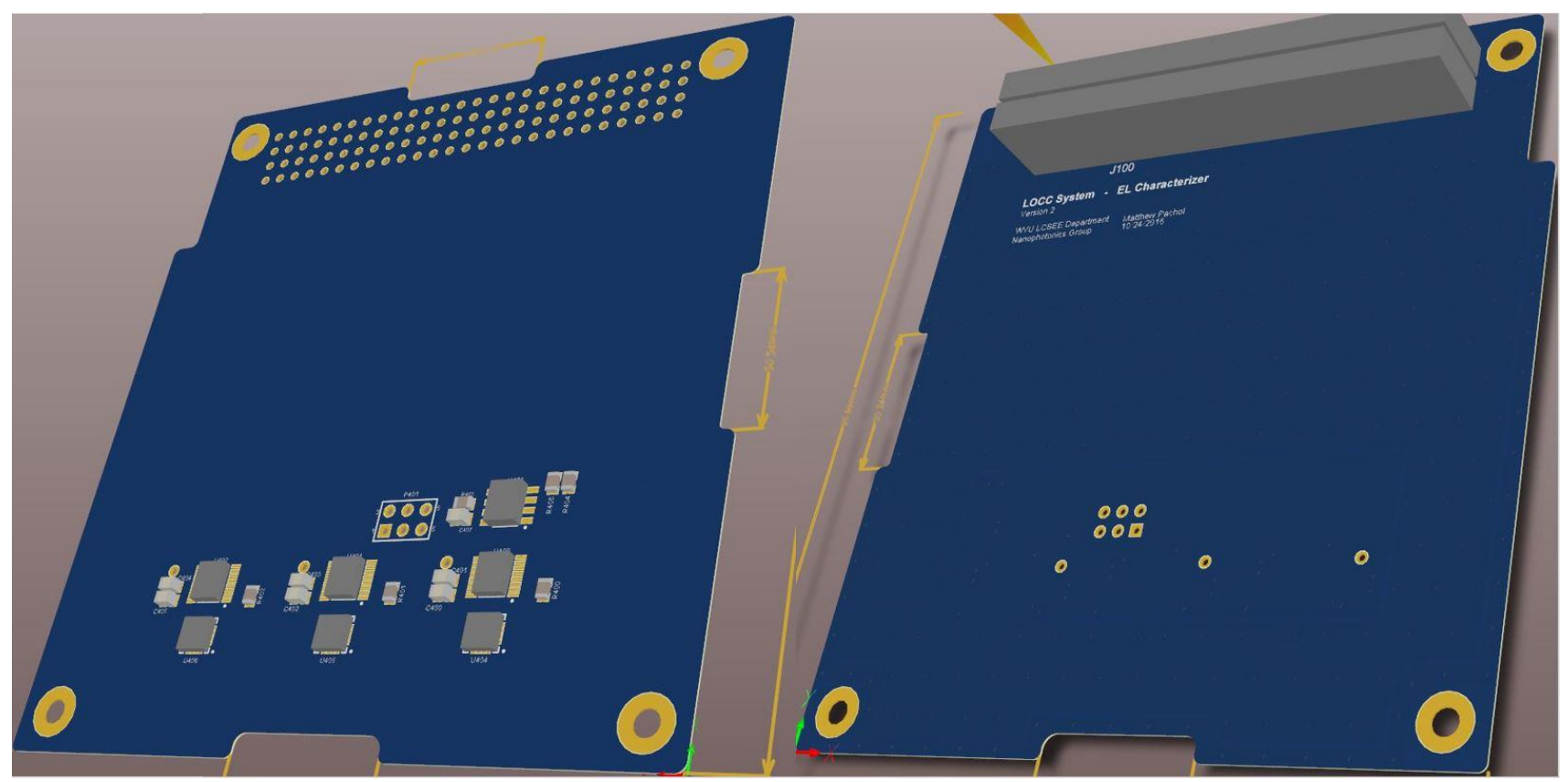

Figure 3. 33: (Left) EL Bottom Side 3D Rendering, (Right) EL Top Side 3D Rendering 


\subsection{Firmware}

This section describes the interfacing along with firmware operation for control. This section also describes, software flow, major functions, and key values to be evaluated regarding the coupling of hardware and software. Included within this section will be process flow diagrams, to show the operations of experiments and communication with the LOCC system. This includes the necessary command codes to initiate specific experiments, retrieve data, set LEDs to be turned on, setting voltage resolution, and retrieve the stored data after completion of the experiment.

\subsubsection{Pin Description}

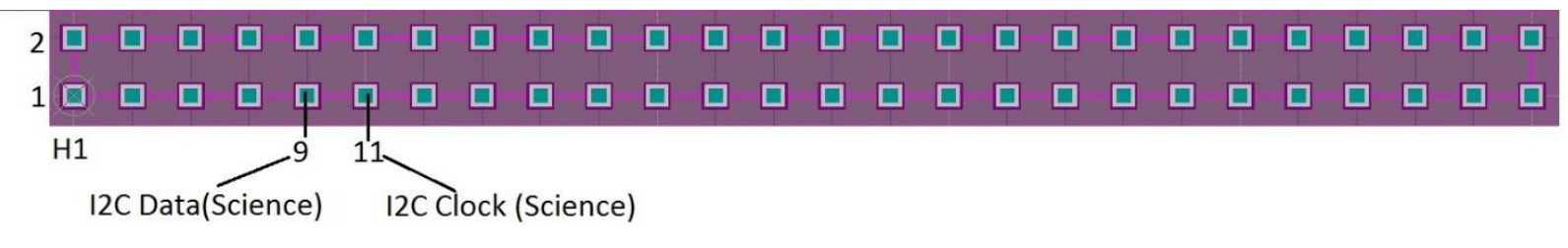

Figure 3. 34: (Top Down View) LOCC Header Pin 1 for STF-1 connection

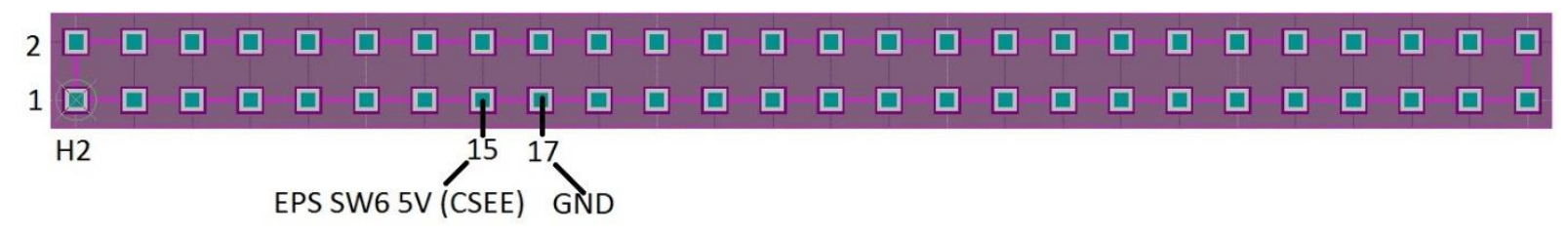

Figure 3. 35:(Top Down View) LOCC Header pin 2 for STF-1 connection

To reiterate the connections from Section 3.2.2, communication and power to the LOCC system is done via the header pins. These connections can be made by simply plugging the proper wires into the labeled slots. 


\subsubsection{Process Flow Diagram}

Figure 3.36 illustrates the flow diagram of the LOCC firmware. Upon switching on power to the LOCC system, the flight computer will confirm with LOCC that the connects are made by receiving a confirmation bit. If the bit is not shared between the system, both the flight computer application and firmware will attempt to resend the confirmation bit. LOCC is now ready to be sent command codes to begin processing of experiments. This is done with command codes found in Table 3.8. The LOCC command codes are read in as hexadecimal values and correspond to written subroutines regarding running experiments, activating or deactivating LEDs, and transferring data between LOCC and the flight computer. Once the command codes are send and experiment runs, data can be sent back to the flight computer using the given command codes. Once the transfer is complete and all desired experiments have been ran, LOCC can then be shut down. 


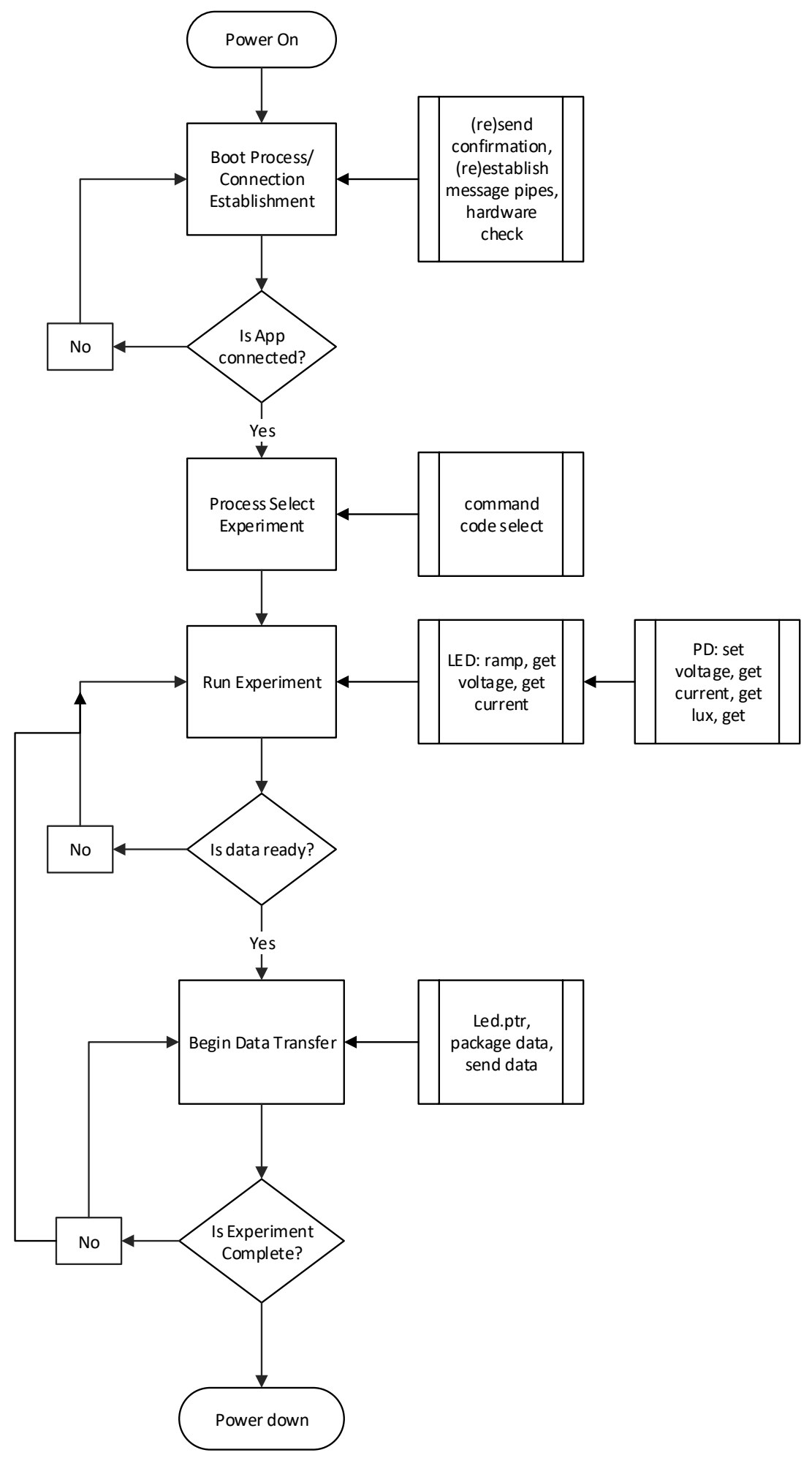

Figure 3. 36: Process flow diagram for experiments of LOCC system 


\subsubsection{Libraries}

Below, a list of libraries used in the operation of the LOCC system is provided. These $\mathrm{C}++$ libraries are necessary to include when compiling and installing the LOCC firmware. A description of each of the included libraries is given to show functionality, as well as describe how the library interacts with the LOCC system and various components.

\section{Standard Arduino Libraries:}

SPI - https://www.arduino.cc/en/Reference/SPI

- Include Files: SPI.h

- The SPI standard library allows for MOSI communication between the Arduino Mega and the MCP4901 DAC.

\section{Open-Source Libraries:}

EEPROMex - http://playground.arduino.cc/Code/EEPROMex

- Include Files: EEPROMex.h, EEPROMVar.h

- The EEPROMex library extends the functionality of the standard EEPROM library for storing data on the Arduino Mega's on-board EEPROM.

SoftI2CMaster - https://github.com/todbot/SoftI2CMaster

- Include Files: SoftI2CMaster.h

- The SoftI2CMaster library allows for software-based I2C communications between the Arduino Mega and I2C-enabled devices (INA219, MCP23018, TCS34725, TSL2561). This frees the on-board hardware-enabled I2C bus for other communication purposes. 
MCP23019 BitBang -

- Include Files: MCP23018_bitbang.h

- The driver library used for controlling soft the MCP23018 GPIO expanders. This library makes it possible to control the expanders via Arduino.

Adafruit INA219 - https://github.com/adafruit/Adafruit_INA219

- Include Files: Adafriot_INA219_BitBang.h

- Library used for controlling the calibration of the current sensor for measure current through LEDs.

CubeSat LCSEE -

- Include Files: CubeSat_CSEE.h

- Used for the overall control of the LOCC system. Creates an instance of the system and allows the user to use getters and setters as well as run experiments and enable/disable LEDs.

MCDC04 Bitbang -

- Include Files: MCDC04_Bitbang.h

- Driver used to control the MCDC04 which is connected to the photosensor. This library allows for the setting of registers, controlling integration time, and calibrations. 


\subsubsection{Data Storage:}

Data will initially be stored on the Arduino Mega's 4096 bytes of on-board EEPROM. A set of memory addresses in the EEPROM will be pre-allocated for storing parameters and program states. Listed below are the variable designators for the stored information regarding each LED. These variables are preallocated within the LOCC memory. Storage of information begins with the LED counter that designates the starting recorded data for that LED. Following this, the voltage resolution is set. The voltage resolution parameter is used to set how many voltage steps are taken from $0-5 \mathrm{~V}$. The LED select parameter used 3 bytes to set the active LEDs to be voltage ramped. The LED status bytes let the user know which LEDs have drawn current and show the user which LEDs are working. These memory addresses require a total of 9 bytes of EEPROM storage. The other 4087 bytes of EEPROM are available for experiment data storage.

- Counter: Current LED (1 Byte)

- A counter showing the current LED being used in the experiment.

- Voltage Resolution (1 Byte)

- A parameter used to determine the input voltage interval of the MCP4901 DAC and the number of data points gathered for each LED.

- $\quad$ LED Select (3 bytes, 1 Byte per LED Array)

- A parameter used to choose individual LEDs or combinations of LEDs for use in either experiment.

- LED Status (3 bytes, 1 Byte per LED Array)

- A set of flag bits representing the working status of LEDs 


\subsubsection{Data Packet Description}

Data packets sent from the Arduino will contain complete data from 1-to-many LEDs. The number of LEDs in the data packet and the size of the packet will depend on the voltage resolution of the MCP4901 DAC. The data, timing and experiment frequency are in Tables 3.5 through 3.7.

Table 3. 5: Experiment 1 Data Size (Bytes)

\begin{tabular}{|c|c|c|c|}
\hline \multicolumn{4}{|c|}{ Experiment 1 } \\
\hline $\begin{array}{c}\text { Single } \\
\text { LED/PD (B) }\end{array}$ & Minimum & Nominal & Maximum \\
\hline $\begin{array}{c}24 \text { LED/PD } \\
\text { (B) }\end{array}$ & 848 & 129 & 1026 \\
\hline
\end{tabular}

Table 3. 6: Experiment 2 Data Size (Bytes)

\begin{tabular}{|c|c|}
\hline \multicolumn{2}{|c|}{ Experiment 2 } \\
\hline & Nominal \\
\hline $\begin{array}{c}\text { Single } \\
\text { LED/PD (B) }\end{array}$ & 17 \\
\hline $\begin{array}{c}24 \mathrm{LED} / \mathrm{PD} \\
\text { (B) }\end{array}$ & 408 \\
\hline
\end{tabular}

Table 3. 7:Experimental Frequency of LOCC

\begin{tabular}{|l|l|}
\hline & Experiment Frequency \\
\hline Week 1 & 3 times per day \\
\hline Week 2 & 1 time per day \\
\hline Week 3 & 3 times in 7 days \\
\hline Week 4. & 1 time in 7 days \\
\hline
\end{tabular}


For Experiment 1, the data structure for an individual LED will be as follows:

- $\quad \operatorname{LED}(1$ Byte)

- Voltage (2N Bytes, where $\mathrm{N}$ is the voltage resolution parameter)

- Current (2N Bytes, where $\mathrm{N}$ is the voltage resolution parameter)

For Experiment 2, the data structure for an individual LED will be as follows.

- $\operatorname{LED}(1$ Byte)

- $\quad$ ADC Reading (3*2 Bytes, $\mathrm{X}$ - adc values, $\mathrm{Y}$ - adc values, $\mathrm{Z}$ - adc values)

- Voltage (2 Bytes)

- Current (2 Bytes)

- $\quad$ ADC Reading (3*2 Bytes, $\mathrm{X}$ - adc values, $\mathrm{Y}$ - adc values, $\mathrm{Z}$ - adc values)

It is important to note the reasoning for take multiple readings from the ADC. This is done to examine the dark current values in the photodetectors. Measuring the dark current gives a baseline value to interpret when looking at the expected output. These values will give insight into any damages that may have or may be occurring in the photodetectors. Dark current may increase due to displacement damages seen from irradiation and produce unreliable results.

\subsection{Software Interfacing}

The software section is dedicated to describing the LOCC application and its functionality when interfacing with hardware and firmware. This section will describe the various command code, message IDs, and functionality of the developed application. The block diagrams provided will illustrate the coupling between processes and hardware. Essentially, the firmware handles all internal processes, and the software handles only its internal processes. 
LOCC is interfaced with STF-1's onboard computer which utilizes Core Flight Executive (cFE), which is an application development and run-time environment. The purpose of cFE is to provide core services that include messaging on a software bus, time, events, executive, and table services. These services are built in, and provide users an interface to each service [176]. For future information of availability and usage see [177].

\subsubsection{Software Process Diagram}

Figure 3.37 illustrates the process diagram for software interfacing LOCC with STF-1's flight computer. The software application onboard of the flight computer is designed specifically to communicate with LOCC. This begins with running the application within $\mathrm{cFE}$ and establishing a connection with LOCC. Here, power is enabled to the system and confirmation of communication between the software and hardware is established. Following the command codes from table 3.8, the commands establish a subroutine, or child task, to begin sending commands and/or receiving data. These child tasks are the gateway to communicating with the LOCC system. 


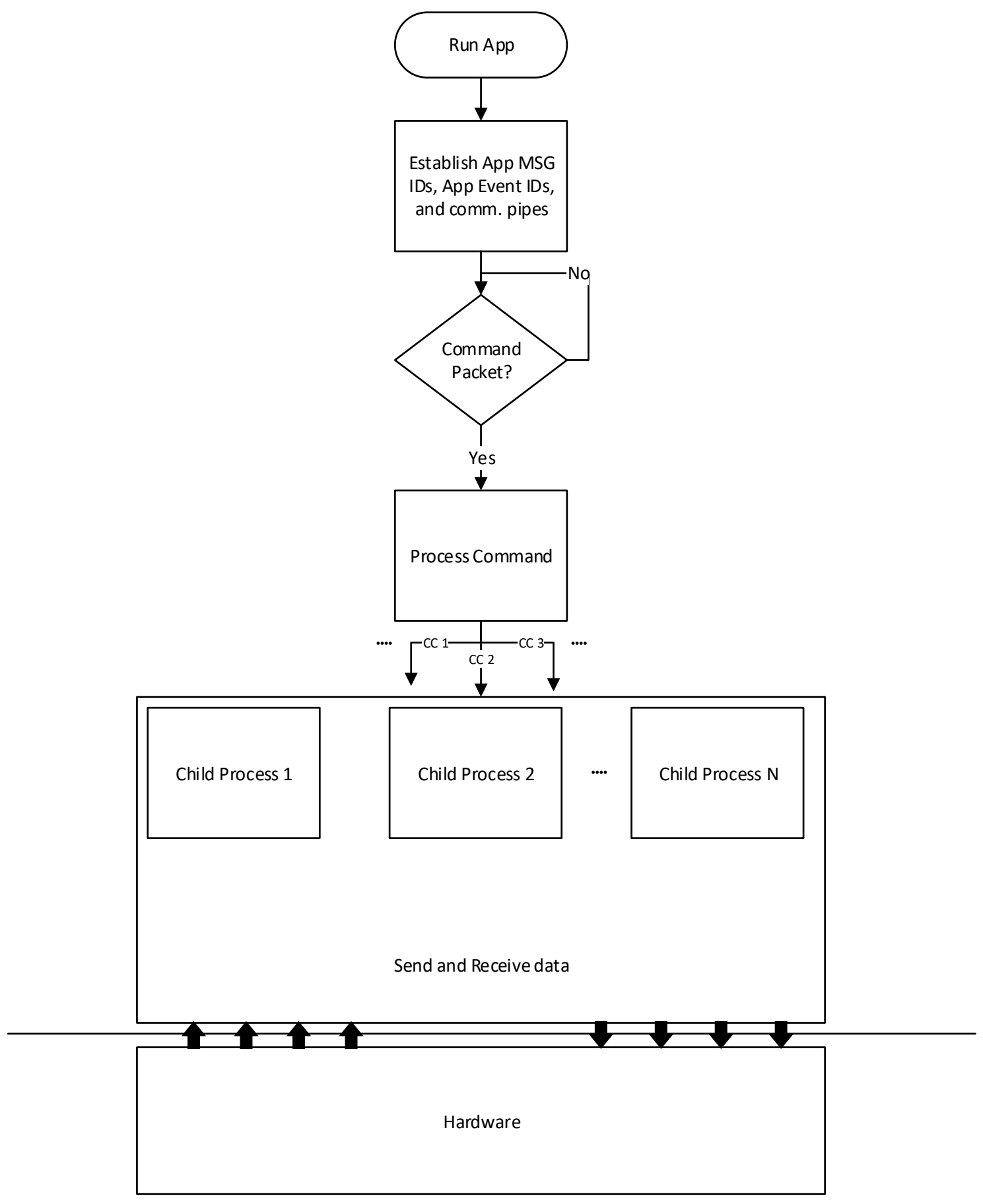

Figure 3. 37: Software Operation Flow Diagram

Tables 3.8, 3.9, and 3.10 provide insight into the command ranges, established message IDs and ground input command codes for command this portion of the experiment. A list of relative functions 
used in the child process is also given for relative information regarding the functionality of the spawned child processes for handling data transfer between software and hardware.

Table 3. 8: LOCC Command and Telemetry Code Ranges

\section{Application Command Range}

Table 3. 9: LOCC Message IDs

\begin{tabular}{|l|l|l|}
\hline Application & App Message IDs & \\
\hline CSEE & CSEE_CMD_MID & 0x1980 \\
\hline & CSEE_SEND_HK_MID & 0x1981 \\
\hline & CSEE_HK_TLM_MID & 0x1982 \\
\hline
\end{tabular}

Table 3. 10: LOCC Command Codes

\begin{tabular}{l}
\hline CC \\
CSEE_NOOP_CC \\
CSEE_REQ_DATA_CC \\
CSEE_RESET_COUNTERS_CC \\
CSEE_STOP_CC \\
CSEE_PAUSE_CC \\
CSEE_RESUME_CC \\
CSEE_EXP1_CC \\
CSEE_EXP2_CC \\
\hline CSEE_RUN_EXP1_CC \\
\hline CSEE_PUB_EXP1_CC \\
\hline CSEE_RUN_EXP2_CC \\
CSEE_PUB_EXP2_CC \\
\hline
\end{tabular}




\subsection{Summary}

This chapter provides and overview of system development for this effort, that include the LOCC system circuit design, PCB layout, and firmware design, and software design. These system components function together to perform experiments for the I-V characterization and the EL characterization of the LEDs. The I-V experiment ramps the voltages of 24 LEDs and records the voltage and current values. This identifies resistivity, turn on voltage, and saturation currents. The second experiment focuses on the EL characteristics of the LEDs. This determines the overall light output and estimation of peak wavelength emission for determining damage constants. Both experiments give information for describing optical and electrical characteristics of the LEDs.

This chapter also describes the LOCC system design, which includes block diagram, circuit design, component description, PCB layout, firmware, and system integration. Each section details the different modules that make up the LOCC system that include power management, communication management, MCU/I-V characterization, and EL characterization. The aim of this

chapter is to provide a detailed overview of the LOCC system and its targeted results. Additionally, specific components are detailed regarding selection and placement that provides ease of use and diagnosis. The overall goal of this chapter is to detail the work done in preparing the LOCC system for integration into the CubeSat platform. 


\section{Chapter 4: LED Fabrication and Packaging}

\subsection{Processing and Fabrication of LEDs}

This chapter describes the fabrication processes of making the custom LEDs for the LOCC system. The chapter is divided into two sections that focus on the fabrication techniques of LEDs and the packaging of the LEDs into chip carriers. Before the LEDs can be processed and fabricated, substrates containing the active regions of the device must be grown. The active regions of the LEDs are typically created using MOVPE techniques [178] [179]. The LED layers are grown on sapphire (Al2O3) substrates that are $430 \mu \mathrm{m}$ thick and a diameter of $50.8 \mathrm{~mm}$. For this work, commercial LED substrates were purchased rather than grown in-house. The characteristics of the LED wafers used in this work are detailed in [180]. After the wafers are grown, the individual LED mesas and contacts are created using successive lithography, etch, and deposition steps. The optimized LED structure can be seen in Figure 4.1. The active region, consisting of periodic InGaN/GaN quantum wells, having a thickness of $200 \mathrm{~nm}$. The blocking layer consists of p-type doped AlGaN, with a thickness of $30 \mathrm{~nm}$. The overall thickness is described in Figure 4.1 The target peak emission is 445 to $475 \mathrm{~nm}$, depending on the concentration of indium dopants and processing. 


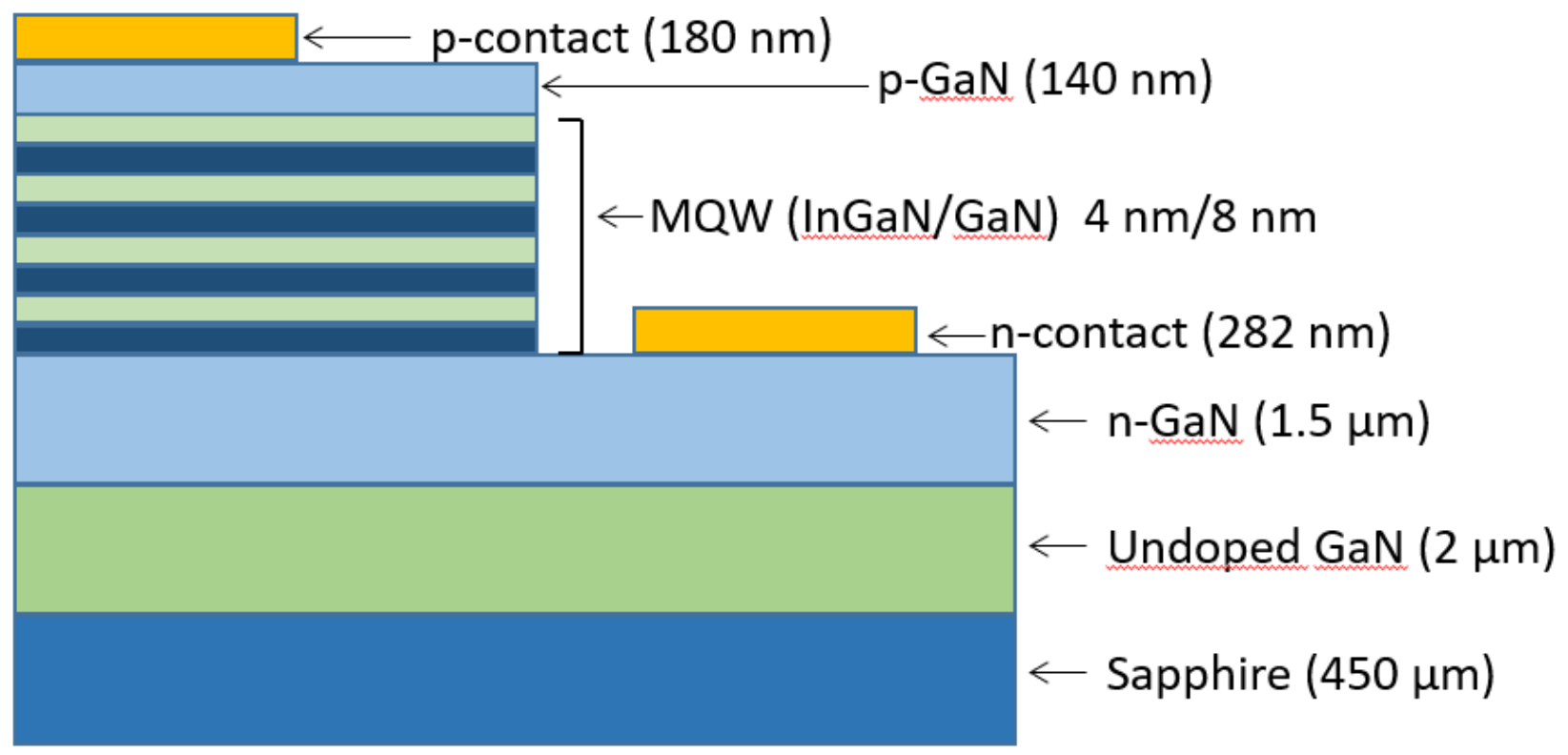

Figure 4. 1: LED Structure

The LED substrates are diced into smaller samples using a diamond scribe to score the rough side of the substrate. Following this, a degreasing step is performed by placing the diced substrate pieces into successive 5-minute ultrasonicated baths of acetone and methanol. The samples are then rinsed using DI water and dried using nitrogen. They are then placed in an oven at $120{ }^{\circ} \mathrm{C}$ for 20 minutes for a dehydration bake. The LED samples then go through an annealing process. This process takes place in a rapid thermal annealer (RTA) at $800{ }^{\circ} \mathrm{C}$ for 5 minutes in a nitrogen environment of $500 \mathrm{sccm}$. This is done to activate the charge carriers and reduce the defects of the p-type GaN [181]. Because the annealing process may form an oxidized layer, the annealing step is followed by an oxide etch to remove this layer. This is done by placing the samples in a sequence of acidic solutions. This consists of a buffered oxide etch (BOE) for 3 minutes, deionized (DI) water rinse for 2 minutes, dilute hydrochloric acid ( $\mathrm{HCl}$ ) with a 1:1 mixture of $\mathrm{HCl}$ and DI water for 1.5 minutes, and finished with another DI water rinse for 2 minutes. Following the removal of oxide layers, another degreasing step 
is performed, and the samples are placed back in the dehydration oven for another 20 minutes to ensure all residues have been removed.

The samples are now fully prepared for mesa patterning and etch. For this, the samples are spun in the Laurel Technologies 600 vacuum spinner hood with AZ4400 photoresists at $4000 \mathrm{rpm}$ with a $1749 \mathrm{rpm}$ acceleration for 30 seconds. This is done to acquire the correct final thickness of 50 $\mathrm{nm}$ of photoresist for exposure and processing. The samples are then placed on a $95{ }^{\circ} \mathrm{C}$ hotplate for 1 minute to evaporate the resist solvents. The samples are now ready for patterning. Using a mask aligner, Suss Microtech MA6 Mask Aligner at $320 \mathrm{~nm}$, photolithography is performed to expose the pattern on the photoresists at an exposure dosage of $250 \mathrm{~mJ} / \mathrm{cm}^{2}$. The exposed AZ4400 can then be developed. Note, the AZ4400 photoresist is a positive resist, meaning the image that is produced from exposer is the same as the photomask. The photomask used was originally designated the bonding LED Mesa mask. Figure 4.2 illustrates the patterning process of the LED mesa.

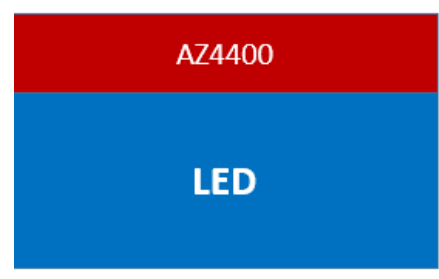

Spin Coating
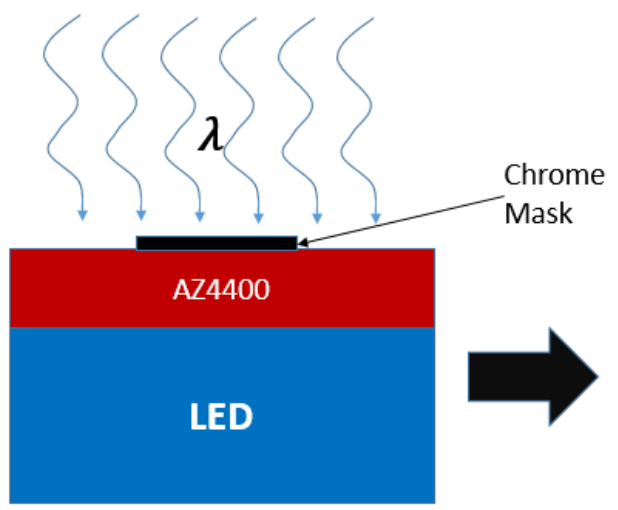

Photolithography

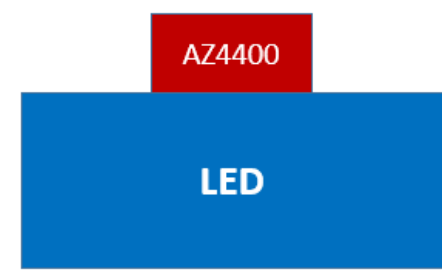

Development

Figure 4. 2: Mesa Patterning Step

AZ 400K developer is diluted with DI water in a 1:3 ratio and developed the exposed resist for about 1.5 minutes. Since AZ 4400 is a positive photoresist, the exposed pattern should be an exact replica of the photomask. Following the development of the mesa pattern, the photoresist is then hard baked 
in the oven at $120{ }^{\circ} \mathrm{C}$ for 20 minutes, which hardens the remaining photoresist and prepares it for the next step of processing, reactive ion etching (RIE). Multiple samples can be placed into the reactive ion etching chamber for processing. Samples are placed onto a silicon wafer by photoresist mounting and placed into the vacuum chamber. To perform the photoresist mounting, a silicon wafers is covered in AZ4400 photoresist and placed in the spinner hood. This is then followed by a hard bake. After the hard bake of the samples and wafer together, the samples are now ready to be placed into the RIE. The mesa structures are created by etching away layers of the LED substrate, exposing the active region of the LED. The Trion Minilock III Inductively coupled plasma (ICP) RIE etching is used to etch the materials. The etching chamber is filled with boron trichloride $\left(B C l_{3}\right)$ at $10 \mathrm{sccm}$ flow, and chlorine $\left(\mathrm{Cl}_{2}\right)$ at $20 \mathrm{sccm}$ flow. The pressure of the chamber is kept at $10 \mathrm{mT}$. The RIE and ICP power are applied to the chamber and the gasses generate a plasma that can begin etching and lifting away of materials. This process is performed for 120 seconds to achieve an etch depth of 500 to $800 \mathrm{~nm}$. The etch depth is governed by other ICP RIE etching parameters such a helium (He) flow, chamber pressure, DC bias, etc. This can produce varied results between $600 \mathrm{~nm}$ to $900 \mathrm{~nm}$ mesa heights over time. As long as the MQWs are exposed during the process, these results will yield functional LEDs. Once the mesa etch is complete, the photoresist can now be stripped from the samples. This is done by placing the samples and silicon wafer in in AZ 300 T stripper for 1 hour at a temperature of $100{ }^{\circ} \mathrm{C}$. Figure 4.3 illustrates the mesa etching process. 


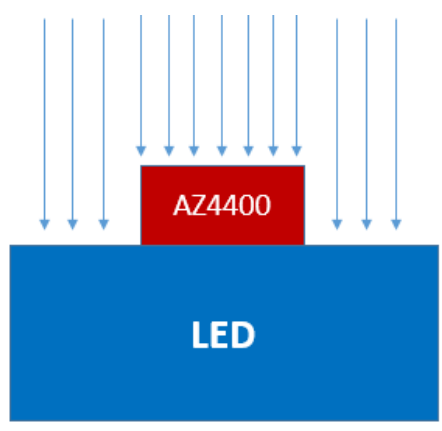

ICP RIE Etch

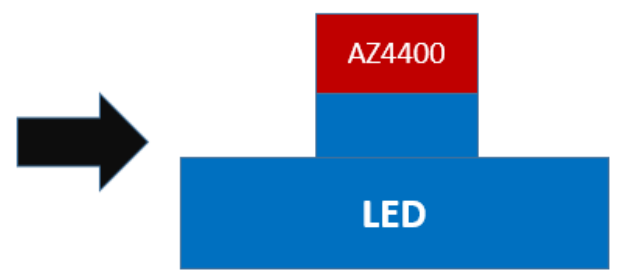

Post Etch

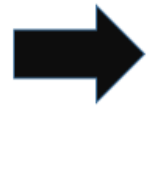

LED

Post Strip

Figure 4. 3: Mesa Etch Processing and PR strip

Following the photoresist strip, the samples undergo another degreasing step and dehydration bake for 20 minutes. The samples are now ready for the next step of processing, p-contact patterning onto the top of the mesa.

For this patterning step, photoresist AZ 5214 E-IR is used. Using the spinner hood, the photoresist is spun at $4000 \mathrm{rpm}$ with an acceleration of $1749 \mathrm{rpm}$ for 30 seconds. This is followed by a solvent evaporation at $95{ }^{\circ} \mathrm{C}$ on a hot plate for 1 minute. The mask aligner is used again with the pcontact mask with an exposure dosage of $50 \mathrm{~mJ} / \mathrm{cm}^{2}$. Following the exposure, the samples are baked at $120{ }^{\circ} \mathrm{C}$ for 2 minutes for the polymer to form crosslinking within the photoresist. A second image reversal flood exposure is then performed, using the OAI UV Flood exposure system at $365 \mathrm{~nm}$, to exposing the samples to a dosage of $2000 \mathrm{~mJ} / \mathrm{cm}^{2}$. Once this exposure is completed, AZ $300 \mathrm{MIF}$ developer is used for 35-45 seconds to reveal the new pattern for the p-contact. Figure 4.4 shows the steps for metal p-contact patterning. 


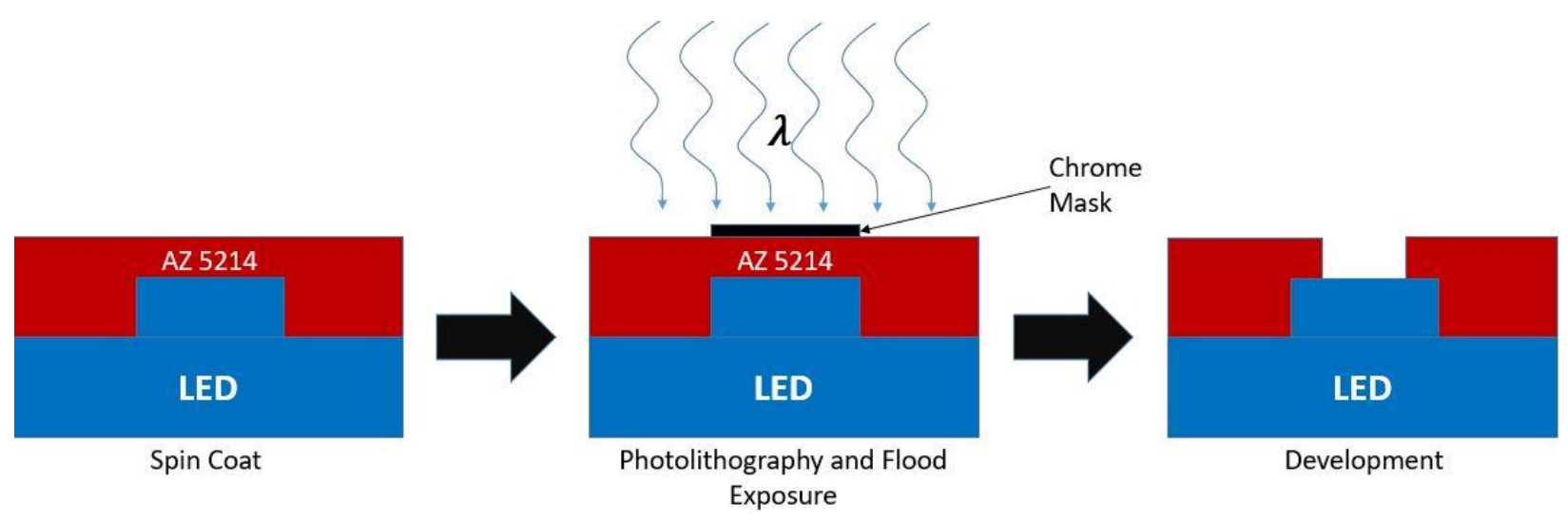

Figure 4. 4: Metal Patterning Process from photolithography

Once the patterning has been completed, the metal and nickel p-contact is ready to be created on top of the LED mesa using the KJL E-Beam for electron beam metal evaporation deposition. The first layer of the contact is a $30 \mathrm{~nm}$ deposition of nickel, followed by a deposition of a second $150 \mathrm{~nm}$ layer of gold. These ohmic contacts were previously developed in [182]. After metal evaporation, a liftoff process is performed. This is done with the ultrasonicator, acetone, and methanol for 5 minutes. This removes the excess gold and photoresist, and leaves the p-contact attached to the mesa. Figure 4.5 shows the metal deposition steps for the p-contact.

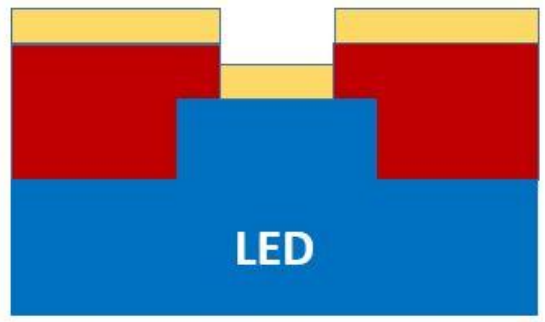

Deposition of Metal

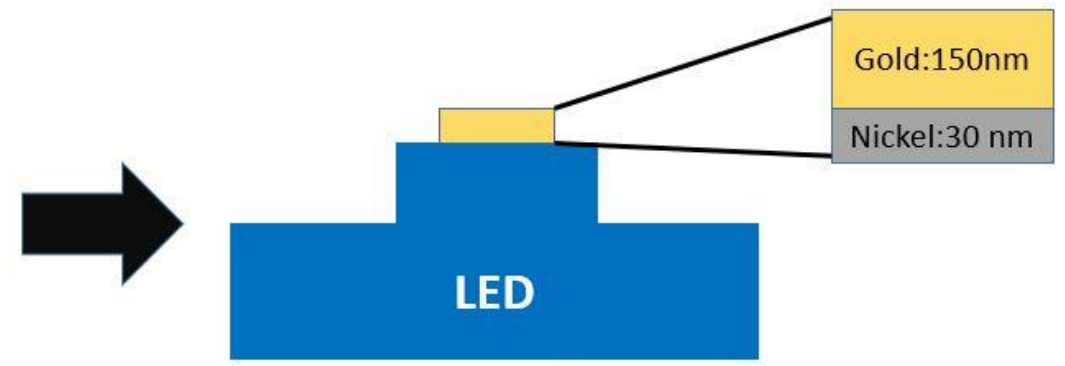

Post Liftoff

Figure 4. 5: Metal Evaporation and liftoff for p-contact 
Before $\mathrm{n}$-contact patterning, another degreasing and dehydration phase is performed to remove residue that main remain after the previous process. The n-contact process is the same as the p-contact patterning process. AZ 5214 E-IR is spun onto the samples at $4000 \mathrm{rpm}$ at an acceleration of $1479 \mathrm{rpm}$ for 30 seconds. This is followed by an evaporation bake at $95{ }^{\circ} \mathrm{C}$ for 1 minute. Using the mask aligner, the photoresist is again exposed to a dosage of $50 \mathrm{~mJ} / \mathrm{cm}^{2}$, and followed by another bake at $120{ }^{\circ} \mathrm{C}$ for 2 minutes for promotion of polymer crosslinking. Another round of flood exposure is used at $2000 \mathrm{~mJ} / \mathrm{cm}^{2}$ dosage for image reversal. The n-contact pattern is then developed using AZ 300 MIF for 30-45 seconds.

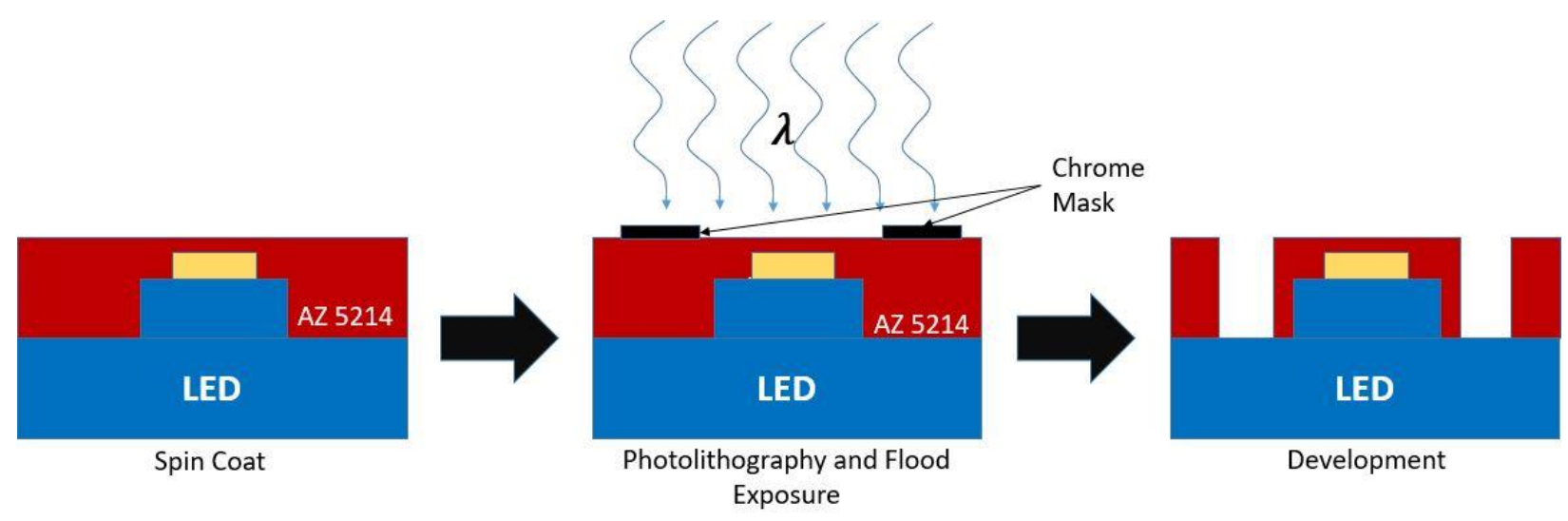

Figure 4. 6: n-contact Patterning Process from photolithography

After development, the n-contact can be deposited using the same method of electron beam evaporation that was used to create the $\mathrm{p}$-contact. The $\mathrm{n}$-contact varies in metals used. The $\mathrm{n}$-contact layers consist of an initial $2 \mathrm{~nm}$ layer of titanium followed by $100 \mathrm{~nm}$ of aluminum, another $30 \mathrm{~nm}$ of titanium, and finally $150 \mathrm{~nm}$ of gold. These materials are layered to promote adhesion between the metals as well as the n-doped $\mathrm{GaN}$ material and form an ohmic contact. After metal deposition, liftoff is performed, and another degreasing and dehydration phase is recommended. Figure 4.7 illustrates metal deposition and liftoff for the n-contact. 


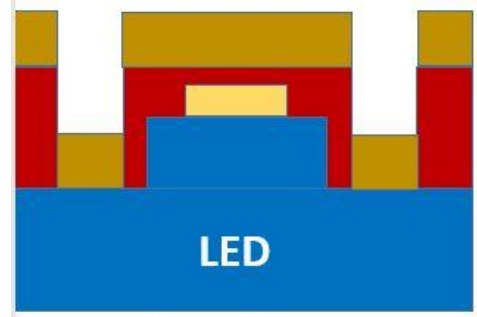

Deposition

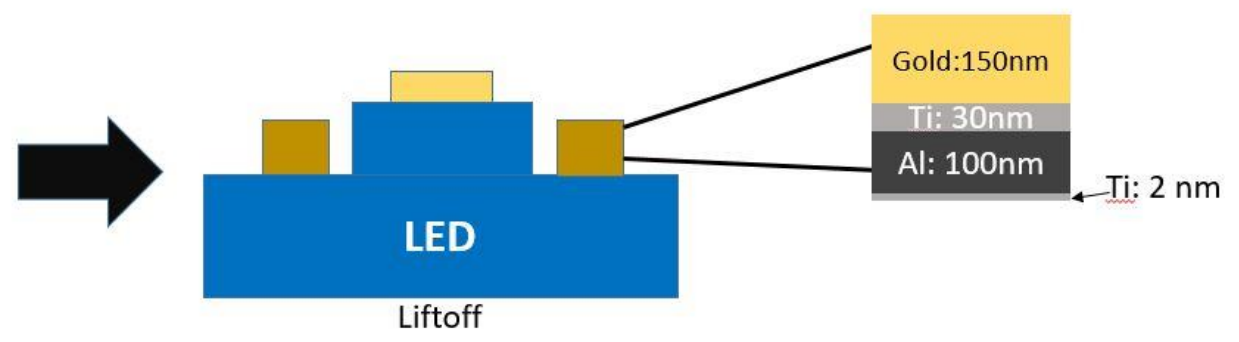

Figure 4. 7: n-contact metal deposition and liftoff

Following the liftoff stage of the samples, the post-process anneal is performed. The RTA is used again for this process. Each sample is placed inside the AnnealSys AS-Micro Rapid Thermal Annealer chamber and annealed at $600{ }^{\circ} \mathrm{C}$ for 3 minutes in air. This process will fuse the layers of the metal contacts together, as well as fuse the metal onto the LED substrate forming a cohesive bond of all the components. The LED is now operational and ready to be powered on. However, because the LEDs need to be packaged to fit into the LOCC, system bonding pads must be added to the contacts to ensure proper bonding from the chip carrier to the LED using gold wire.

The bonding pad layer follows the same procedures as the $\mathrm{p}$ - and n-contacts, utilizing AZ 5214, solvent evaporation, photolithography, crosslinking bake, flood exposure image reversal, and development (see previous sections on p-contact and n-contact patterning). Bonding pads require the use of an alternative deposition technique to improve adhesion to the underlying layers, sputtering. For this, a CVC 610 DC Magnetron Sputtering Station is used sputter a layer of gold deposited for $\sim 6.5$ minutes, producing a $600 \mathrm{~nm}$-thick layer on top of the $\mathrm{p}$ and $\mathrm{n}$ contacts. Figures 4.8 and 4.9 illustrate the patterning and metal deposition of the bonding pads. 


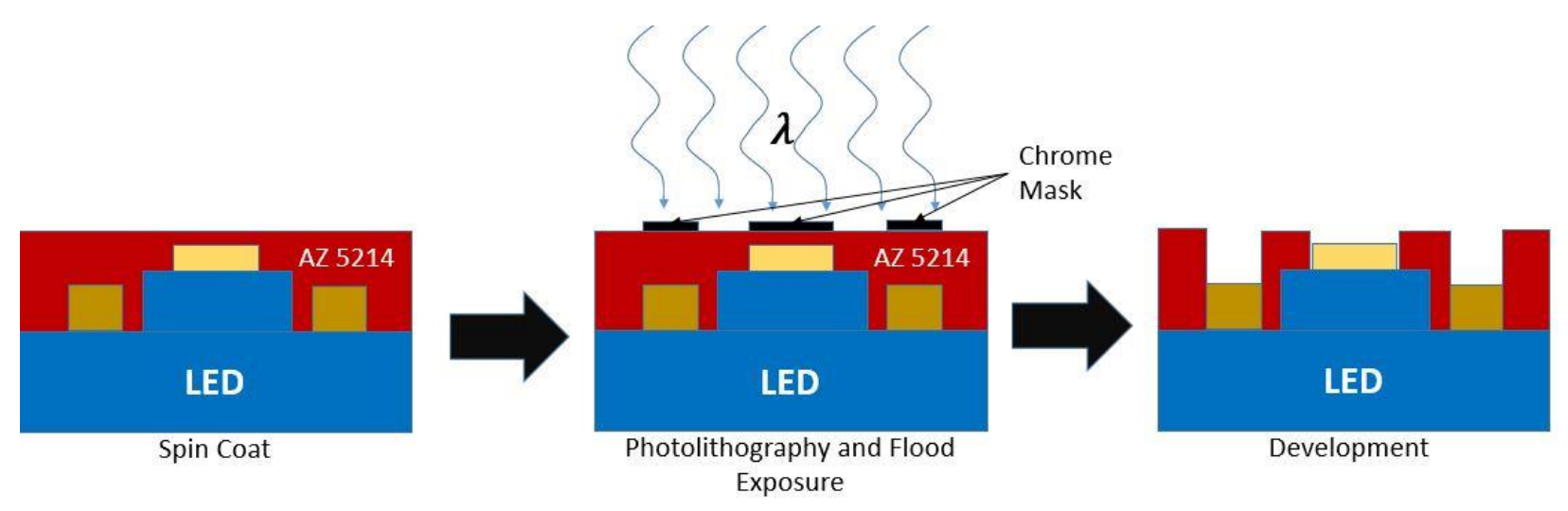

Figure 4. 8: Bonding Pad Patterning

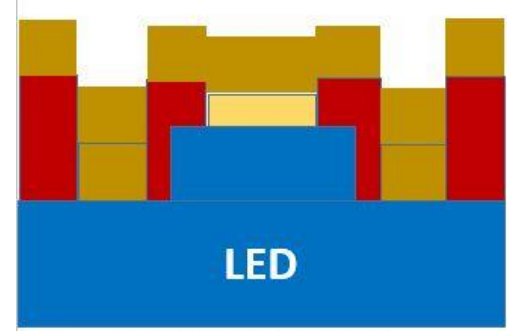

Sputter Deposition

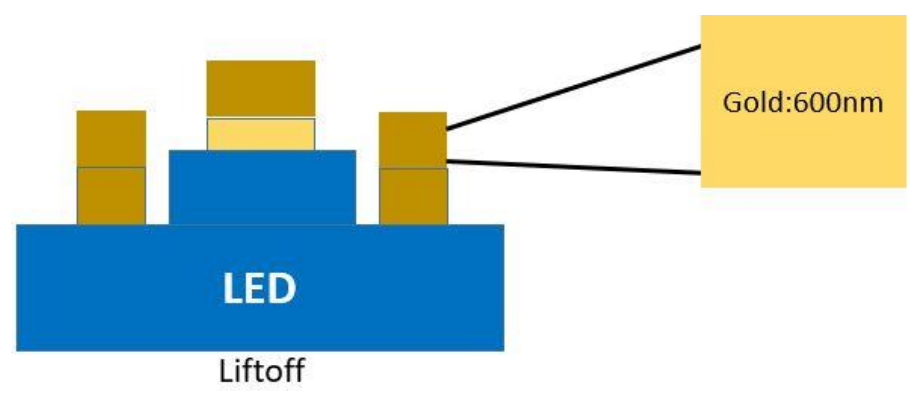

Figure 4. 9: Bonding pad metal deposition

Following the deposition of the gold bonding pad, the LED is now ready to be packaged. The packaging process is detailed in the next section, Section 4.2. Figure 4.10 shows a 3D illustration of a processed LED. 


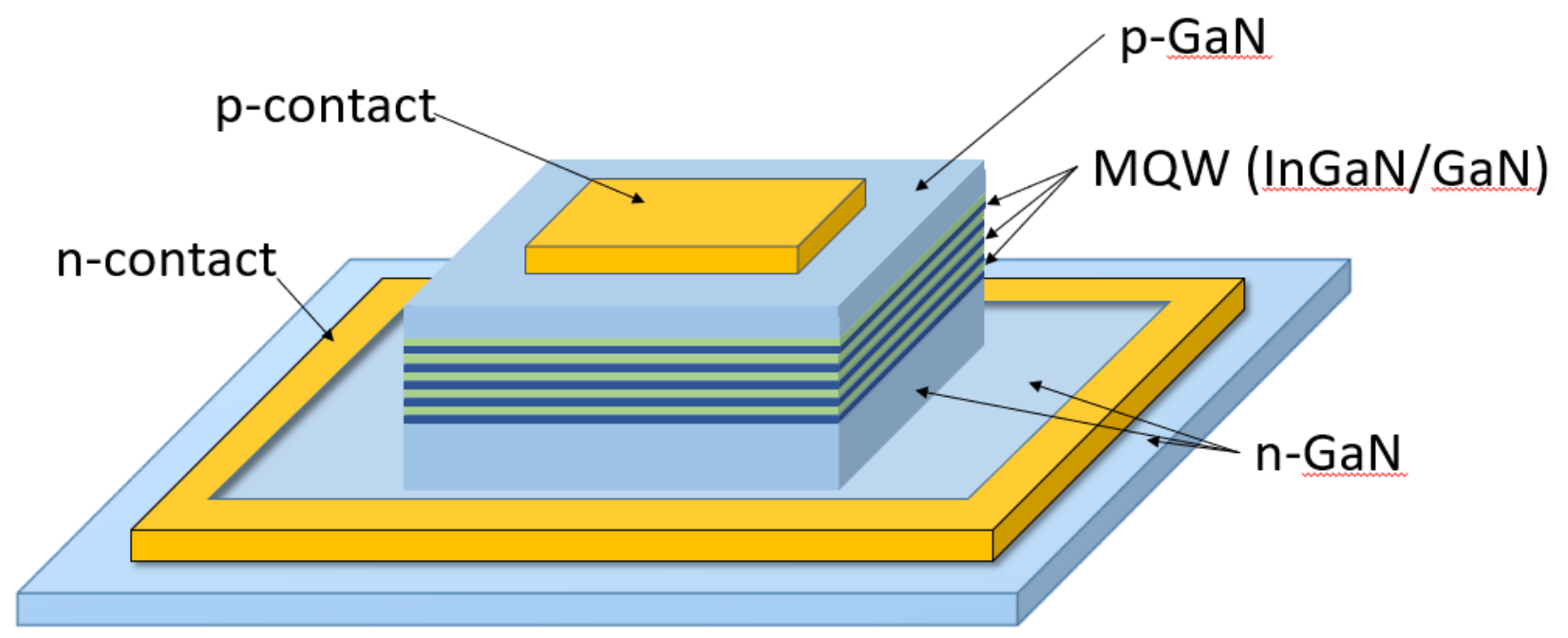

Figure 4. 10: 3D illustration of fabricated LED

\subsection{Integration and Packaging of LEDs}

For the LEDs to be implemented into the LOCC system for characterization, they must first be cut into smaller sizes. To do this, a Disco Tech wafer dicing system is utilized to cut the LEDs into 4-by-4 arrays to be placed into the chip carriers. This is done with a hub-style DISCO diamond blade ZH05-SD2000-N1-50 [183]. The blade is aligned to the corner of the sample and the initial cut is made. After the initial cut the blade is incrementally moved to make cuts every $3.5 \mathrm{~mm}$ in the $\mathrm{x}$ direction for 3 cuts. Following the completion of the $\mathrm{x}$-direction, the wafer dicer will then rotate the sample and move in the $y$-direction. From the initial cut, the sample then incrementally moves in the y-direction $3.25 \mathrm{~mm}$ for 3 cuts. Each cut runs for at least $7 \mathrm{~mm}$ to ensure the sample is cut all the way through. Figure 4.11 illustrates the cut lines and dicing of the LEDs into 4-by-4 arrays. 


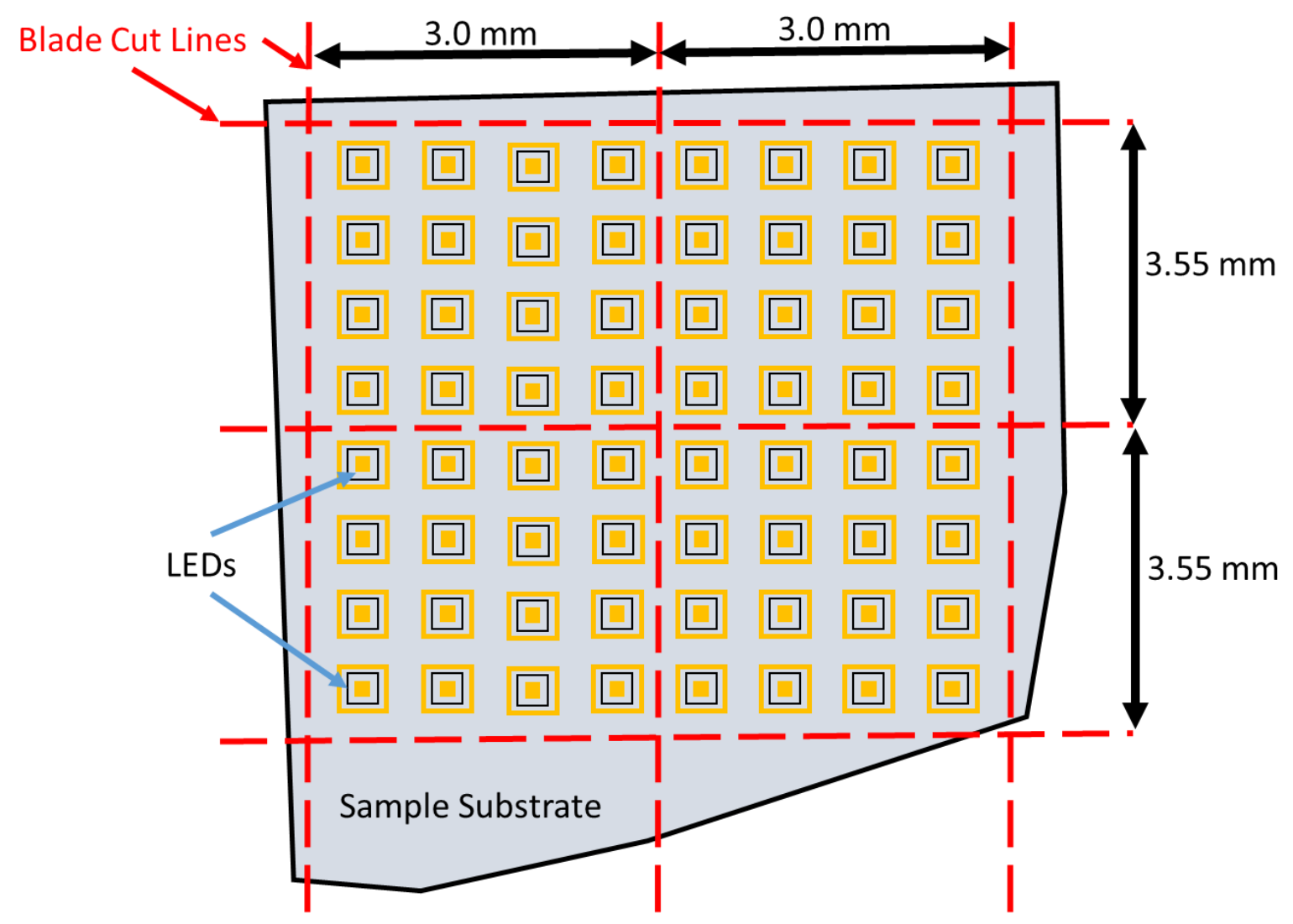

Figure 4. 11: Cut lines and dimensions for sample dicing

Following the dicing of the samples, packaging of the LEDs into the chip carriers can be performed. The diced samples and chip carriers both undergo the degreasing and dehydration phase to clean the diced LEDs of substrate particles from the dicing process, and to ensure the chip carrier has a clean surface for epoxy attachment to the carrier. Following degreasing, EPO-TEK's 353ND [184] high temperature epoxy can be used to secure the LEDs into the chip carrier. This is done by mixing two components together (A and B) with a mixture ratio of $10: 1$ by weight (10 grams of $\mathrm{A}$ with 1 gram of B). The mixed epoxy is then deposited onto the chip carrier in small amounts. One to two small drops are sufficient; adding more will cause over-expansion of the curing epoxy and subsequent creep onto the chip carrier pads and LED bonding pads, leaving them unusable for bonding. After the epoxy has been placed onto the chip carriers, they can be desiccated for 30 minutes 
in a vented desiccator to ensure that all air bubbles have been removed from the epoxy. This step ensures that all air bubbles formed in the epoxy are removed, helping promote strong adhesion between the LEDs and carrier. Once desiccation is complete, the LEDs can now be placed onto the epoxy, pressed in, and placed into an oven to cure for 1 hour at $150{ }^{\circ} \mathrm{C}$. Figure 4.12 illustrates the process of mounting the LEDs.

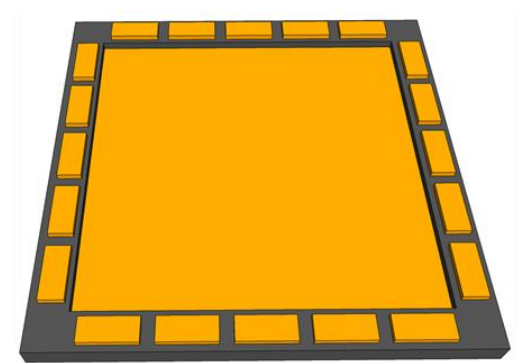

1. Clean chip carrier

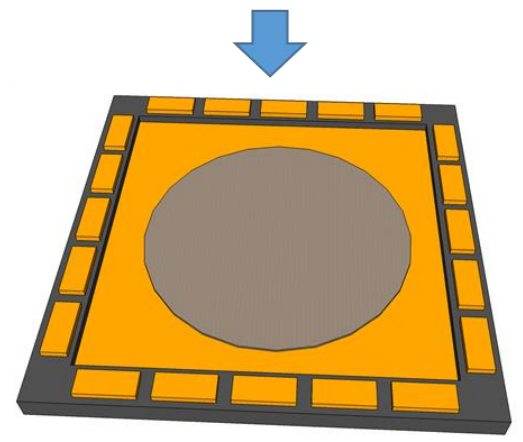

2. Desiccated Epoxy 353 ND

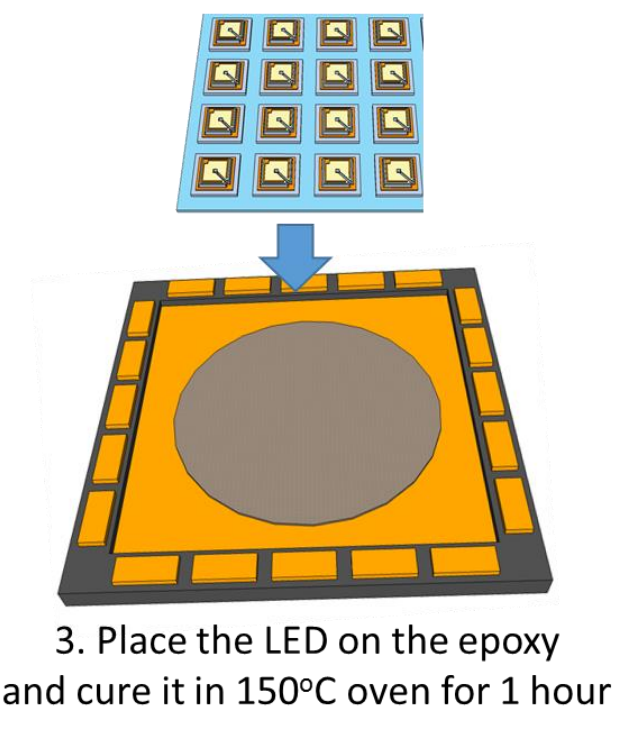
and cure it in $150^{\circ} \mathrm{C}$ oven for 1 hour

Figure 4. 12: Illustration of LED bonding to chip carrier

After epoxy curing is completed, the LEDs are ready to be wire-bonded. The West Bond 74776E wire bonder uses an ultrasonic bonding technique to create traditional wedge/ball bonds. Beginning with the ball bond on the chip carrier pad, the bonding power is set to $200 \mu \mathrm{W}$ for a duration of $150 \mathrm{~ms}$. For the wedge bonding of the LED, the bonding power is decreased to ensure no damage to the contacts. This is done with $120 \mu \mathrm{W}$ of power at a duration of $150 \mathrm{~ms}$. Figure 4.13 shows the bonds that are made with the LEDs to operate properly with LOCC. Note the limitations of the number of 
LEDs that can be bonded. This has left only 8 LEDs to be connected. Here, the red lines represent the positive terminal or $\mathrm{p}$-contact wire bond and black is the negative terminal or $\mathrm{n}$-contact bond.

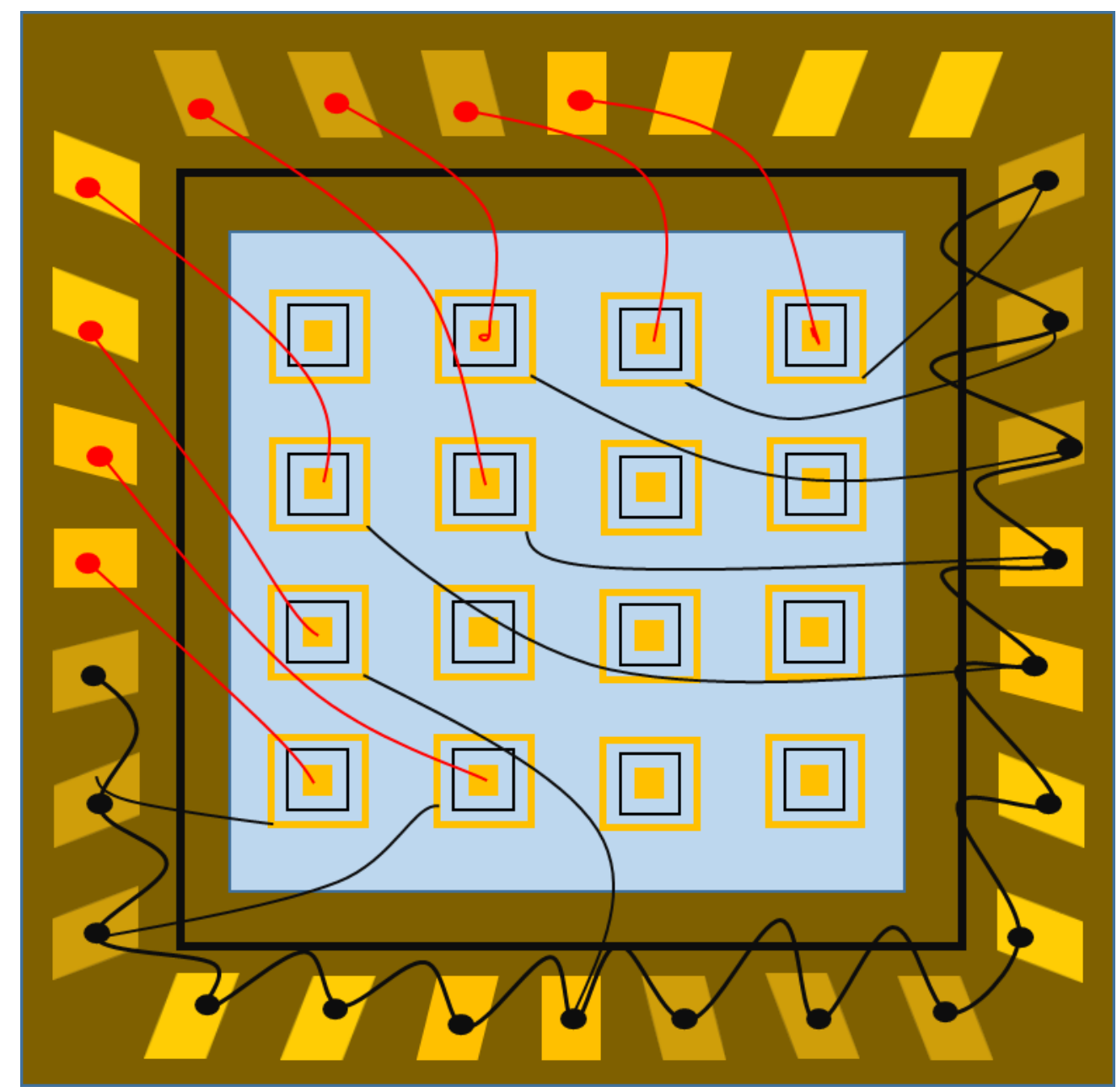

Figure 4. 13: Wire bonding diagram for LOCC compliance

For future design and easier bonding Figure 4.14 gives a recommended configuration for bonding the LEDs to the carrier. The wire bond configuration would require LOCC to have a new design for the assignment of connections. This configuration allows for 12 of LEDs to be connected as well as better connections without the risk of short circuits and misconnections. 


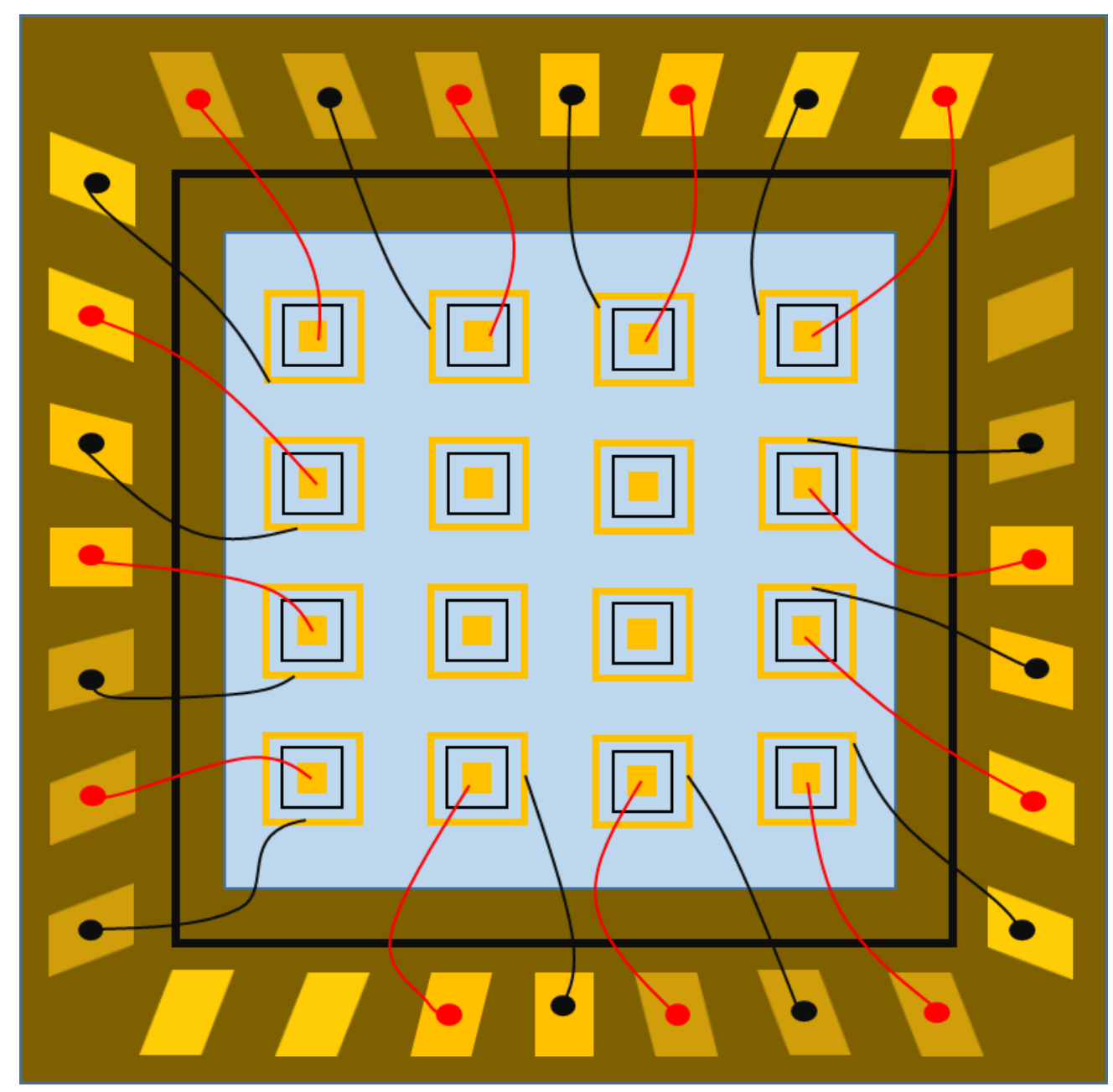

Figure 4. 14: Recommended wire bond configuration.

The final step for completing the packaging process for the LEDs is to apply an epoxy encapsulate to ensure the wire bonds and LEDs stay in place within the chip carriers. This is done using EPO-TEK's 301-2FL low-stress optical epoxy [185] at a mixture ratio of 100:35 by weight. For conservation of epoxy, a mixture ration of 10 grams to 3.5 grams was used. This epoxy is then cured for 3 hours at $80^{\circ}$ C. Figure 4.15 shows the final encapsulation step for packaging the LEDs after wire bonding. 


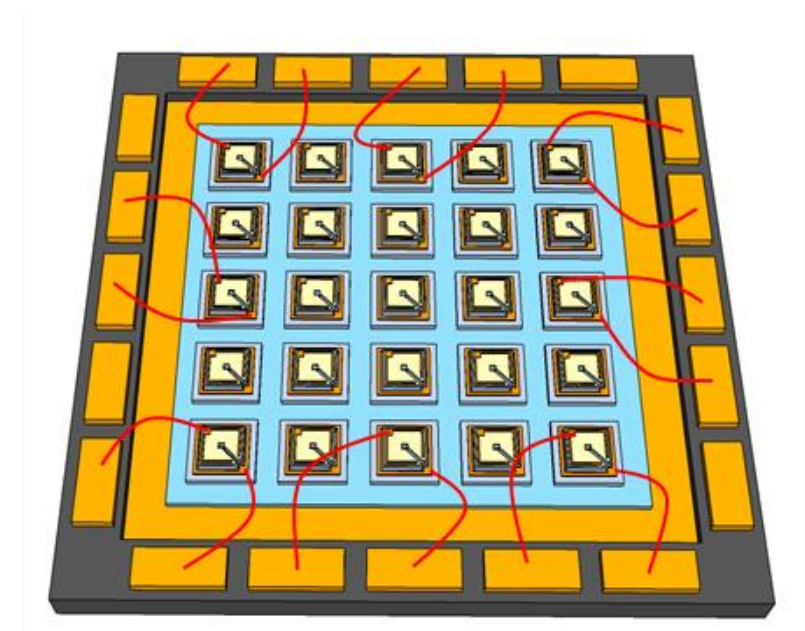

\section{Wire Bond the LEDs}

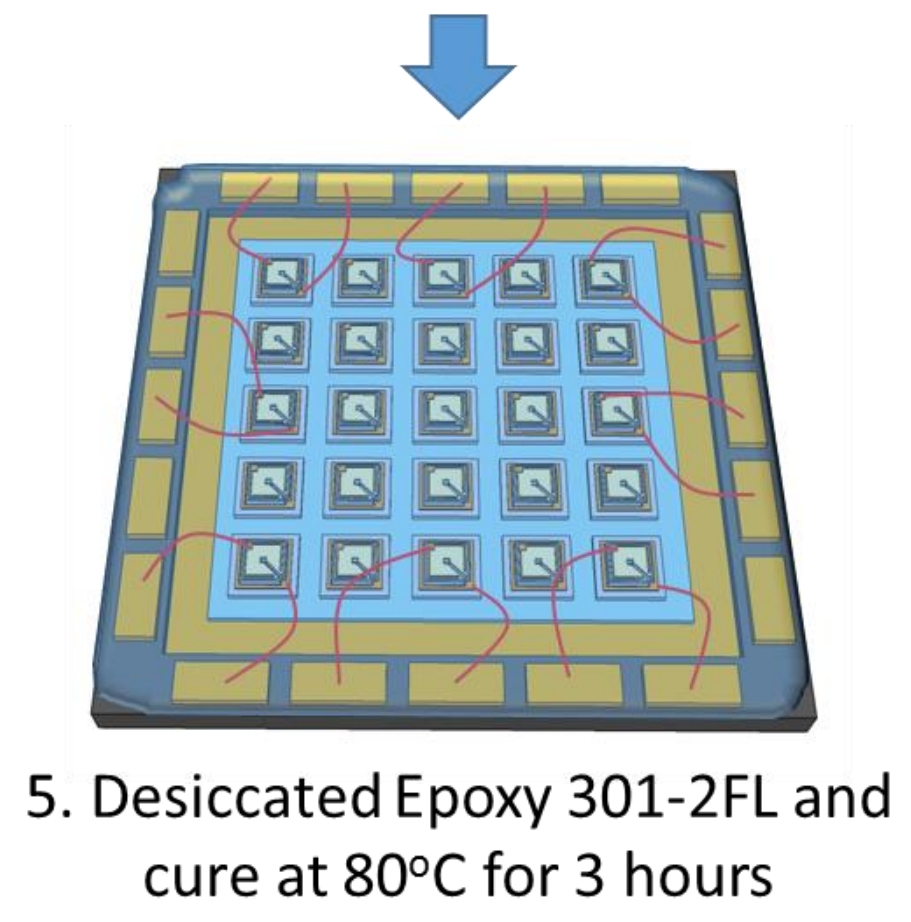

Figure 4. 15: Final Packaging Steps

After a cooling period, the LEDs are now ready to be placed in the LOCC system for testing or characterized with the in-house characterizer. 


\section{Chapter 5: System Characterization \& Testing}

\subsection{Introduction}

This chapter will focus on characterizing the outputs of the LOCC system that consist of I-V ramping results, tristimulus results mapped to CIE 1931 chromaticity diagram, light outputs results, power consumption, and data packet sizes. The results were produced within the assembled CubeSat to show functionality of the LOCC system within STF-1. The experimental data was sent from LOC, to the flight computer, and, finally, transmitted to the ground station computers. The files produced are binary (.bin) files ranging from 900 bytes to 3 kilobytes. Following the download of the binary files, they are translated into their respective text files with float values and plotted using Origin and MATLAB.

In this portion of the work, OriginPro 8 was used to graph and statistically analyze the values produced from the LOCC experiment. Following this, MATLAB was used to determine the corrected tristimulus values for each LED tested, and place them on the chromaticity. From experiment two of LOCC, the light intensity output is determined over all the iterations of the experiment and provided for a baseline observation to be compared to after thermal and irradiation exposure for determining damage coefficients.

Data that is presented in the following chapter has been produced following NASA's vibration and thermal testing of the fully assembled LOCC system, illustrating the capability of the assembly to operate within acceptable ranges after being exposed to extreme conditions. The sub-sections of this chapter cover the generation of I-V curves using the LOCC system, associating them with devices in their respective chip carriers, as well as across the multiple trial runs. This also covers the mean value of current versus voltage of the LEDs in their respective chip carries and their standard deviation as well as the mean values of current versus voltage of each LED and their standard deviations across 
each LED. Next, the correlated LED peak wavelength emissions are estimated and plotted to against a CIE 1931 chromaticity diagram. This chapter will also discuss the resulting light output levels from each LED. The last portion of this chapter will cover the overall recorded power and data costs of the LOCC system.

\subsection{Final Assembly}

The overall LOCC system is assembled to analyze in-house fabricated LEDs. Figure 5.1 shows the final packaging steps and LEDs that will be loaded into LOCC's chip carrier sockets. Figure 5.1(a) shows the initial activation of the LED prior to being diced and epoxied into the chip carrier (as described in the previous chapter). Figure 5.1(b) illustrates a close-up of the wire bonded LEDs to the chip carrier. Figure 5.1(c) and (d) show the encapsulated LEDs following the epoxy cure, ready to be placed into the LOCC system. 


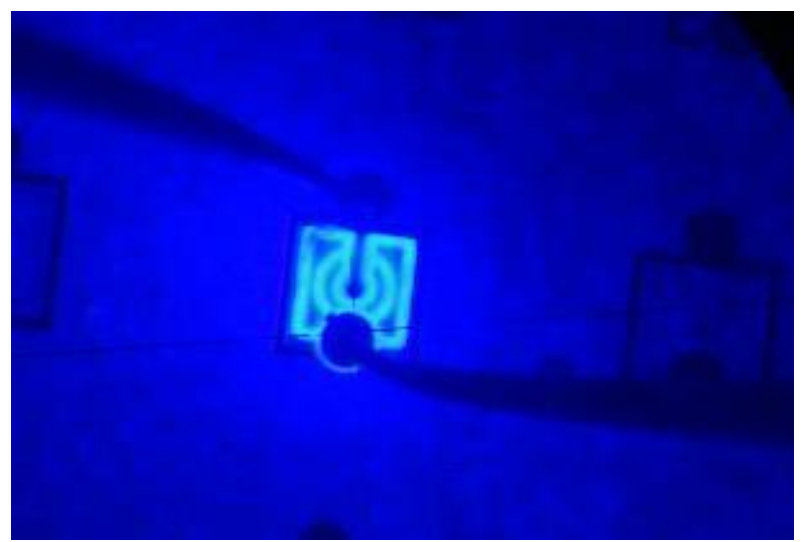

(a)

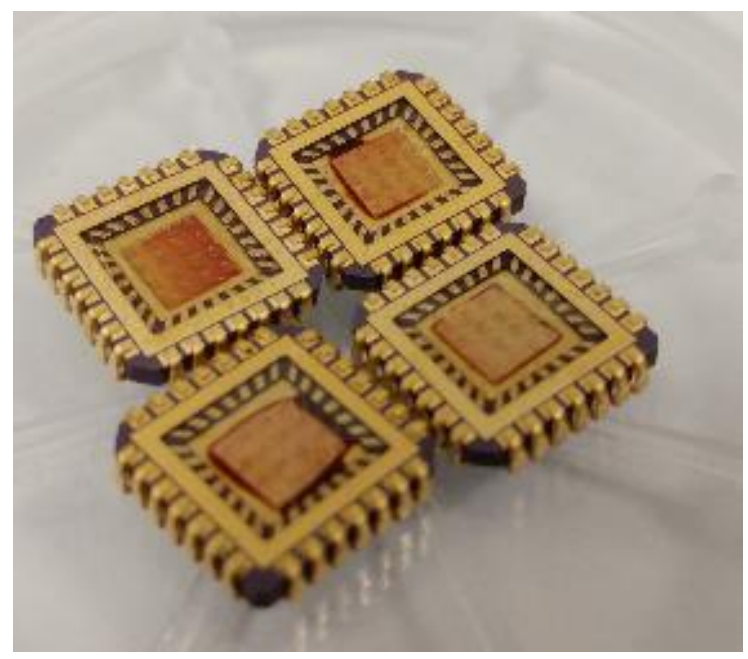

(c)

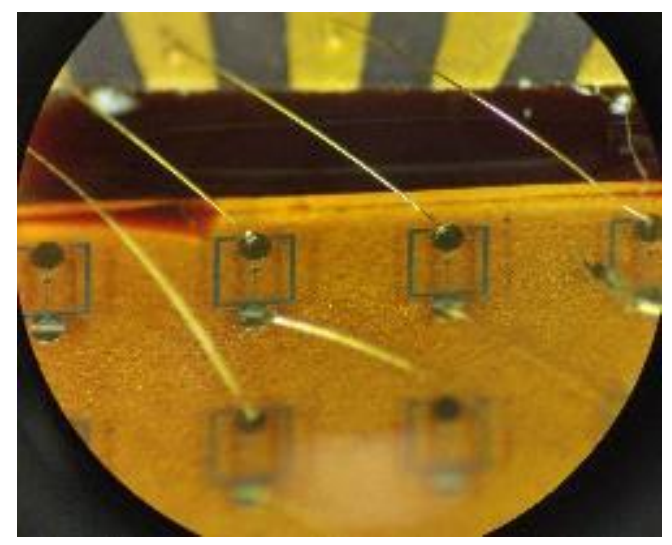

(b)

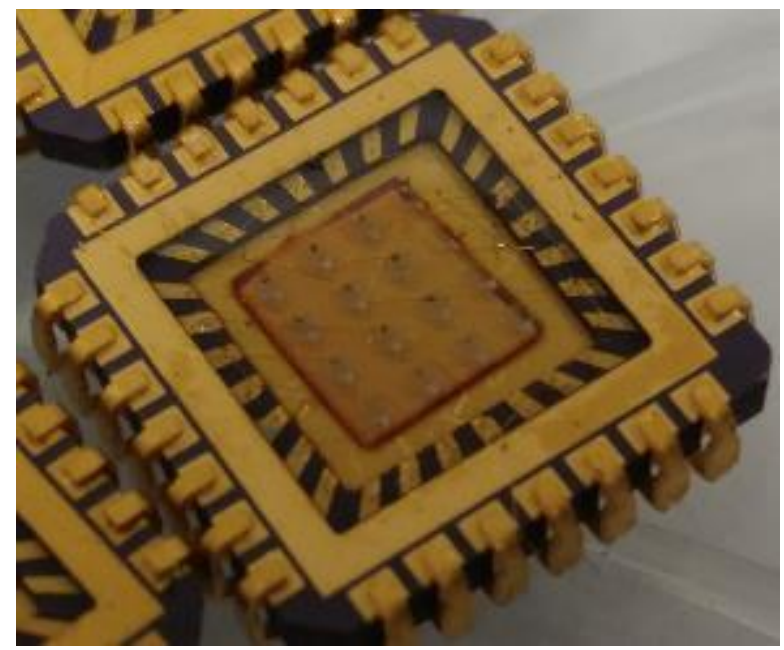

(d)

Figure 5. 1: (a)LED - Pre-package test, (b)Close-up of wire bonded LED, (c) Pre-encapsulation LED chip carriers, (d)Post-encapsulation chip carrier

Figure 5.2 illustrates the final assembly of the LOCC I-V and EL boards. The firmware is uploaded, and the two boards can be connected and integrated into the CubeSat. Figure 5.2(a) shows the bottom side of the I-V characterizer, showing the entirety of the I-V characterization, power and data isolation, and controlling circuitry. Figure 5.2(b) shows the top side of both the I-V and EL characterization module boards. The chip carrier sockets are the only components located on the I-V board side. Figure 5.2(c) shows the LOCC I-V board in operation, enabling an LED and turning it on. Figure 5.2(d) shows a close-up of final assembly of the EL side. 


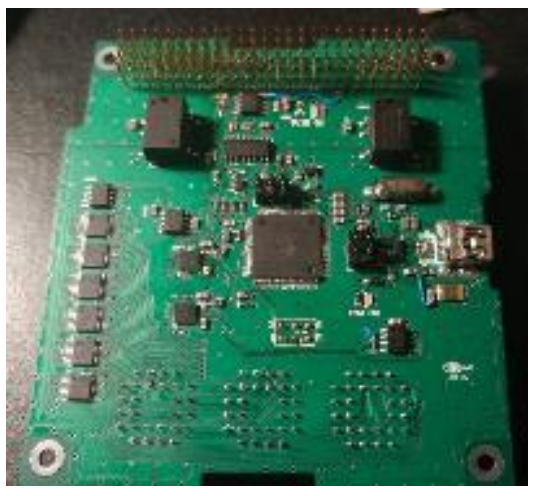

(a)

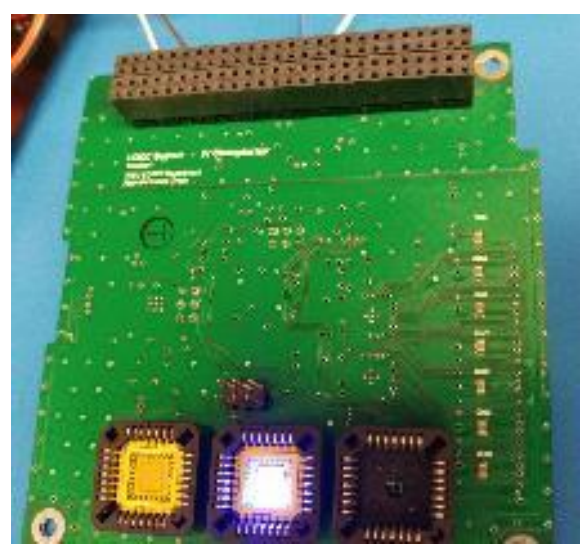

(c)

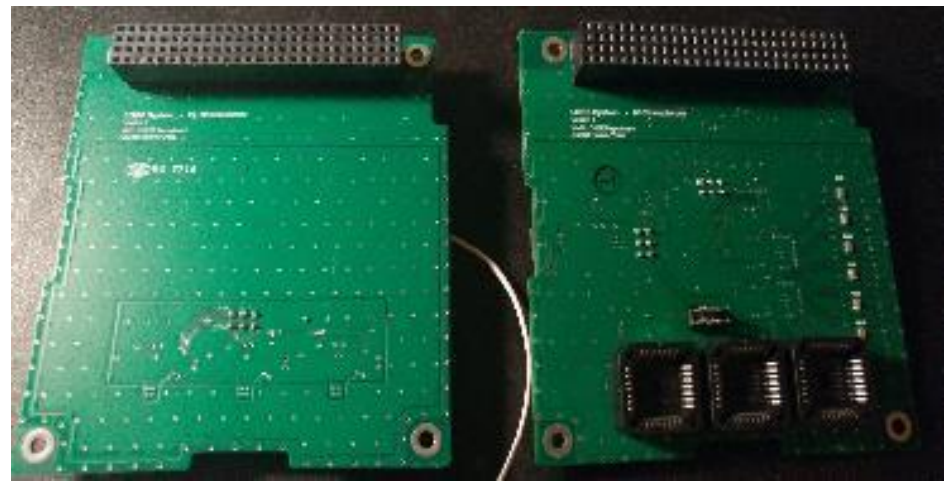

(b)

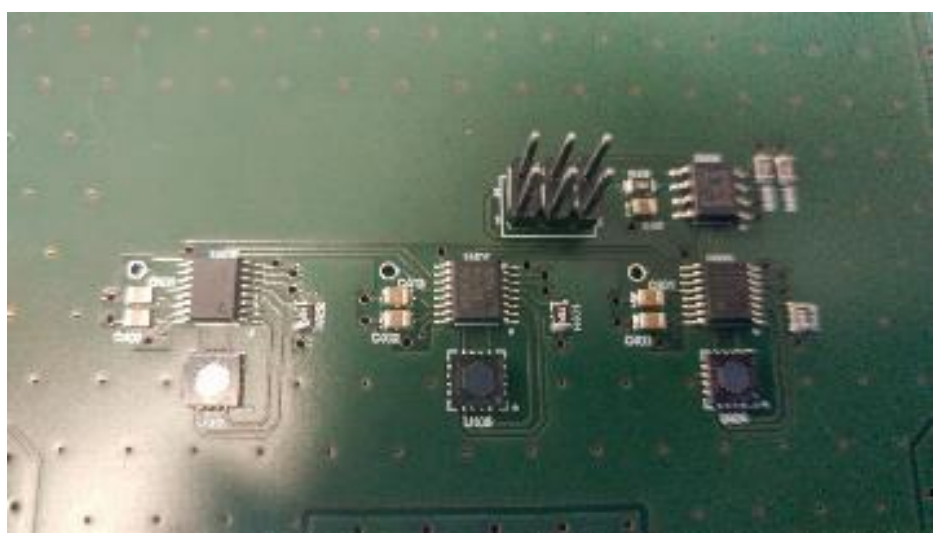

(d)

Figure 5. 2: (a)I-V Characterizer bottom side, (b)EL and I-V characterizer top side, (c)Functional test with lit LED, (d)EL characterizer top side

Figures 5.3 and 5.4 illustrate the connected LOCC system. The boards are pressed together, connecting the center header pins and allowing communication between the I-V and EL boards. This is also how the EL board receives power. 


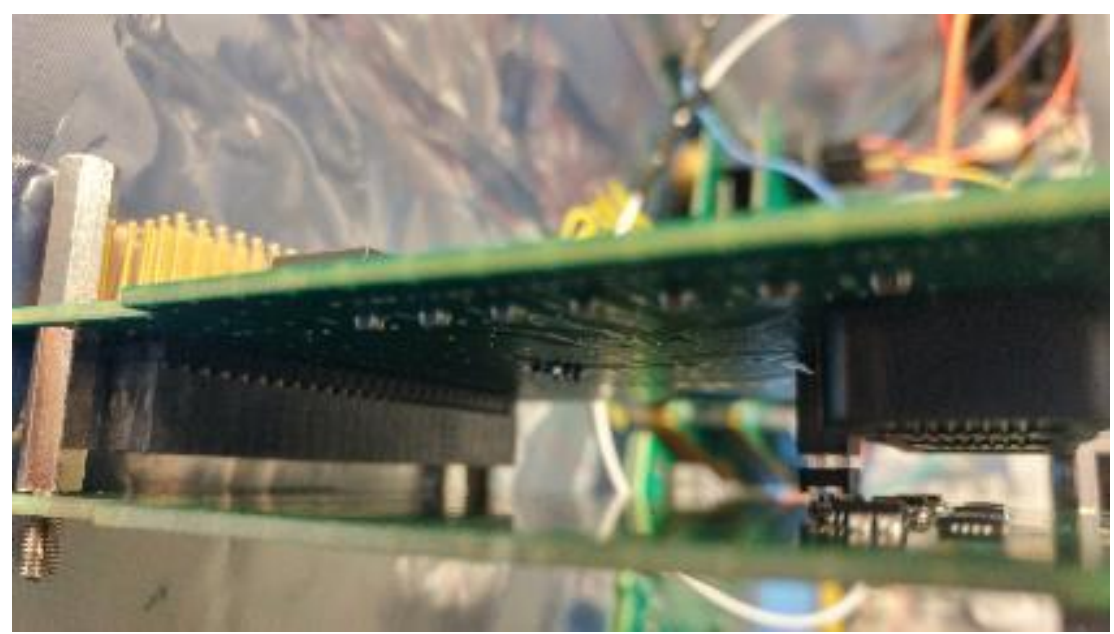

Figure 5. 3: LOCC connected

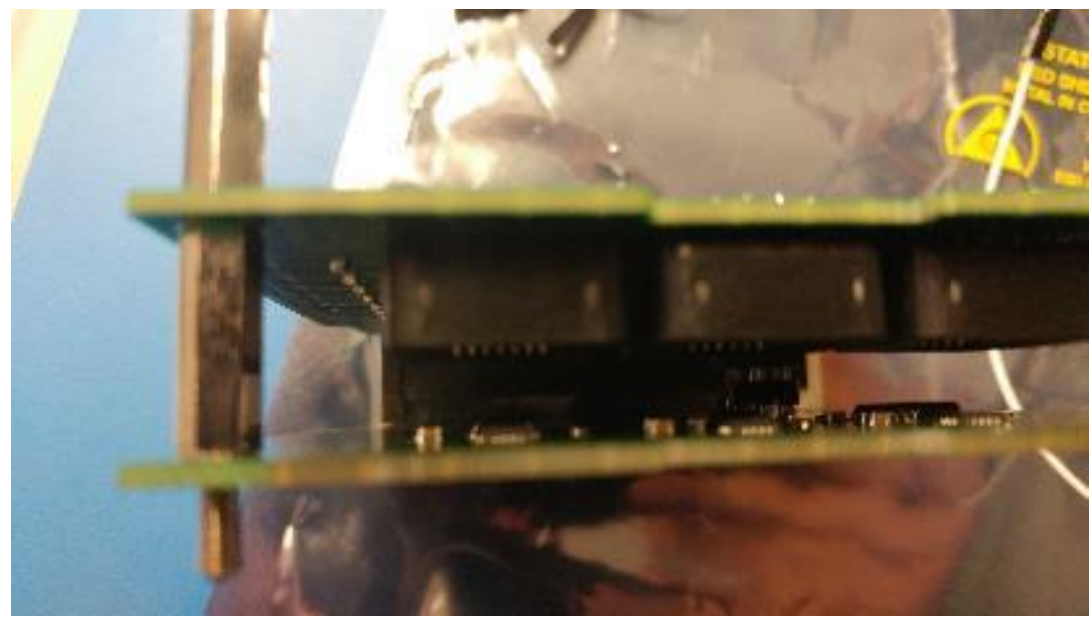

Figure 5. 4: Alternate angle of LOCC connected

Figures 5.5 and 5.6 show the integration testing of LOCC with STF-1 before assembly into the CubeSat Chassis. Power was supplied from the STF-1's battery and monitored for current draw and voltage stability. Additionally, command codes were sent to run LOCC's experiments and retrieve data. This ensured that LOCC was tested and would not interfere with the other existing experiments on-board STF-1. 


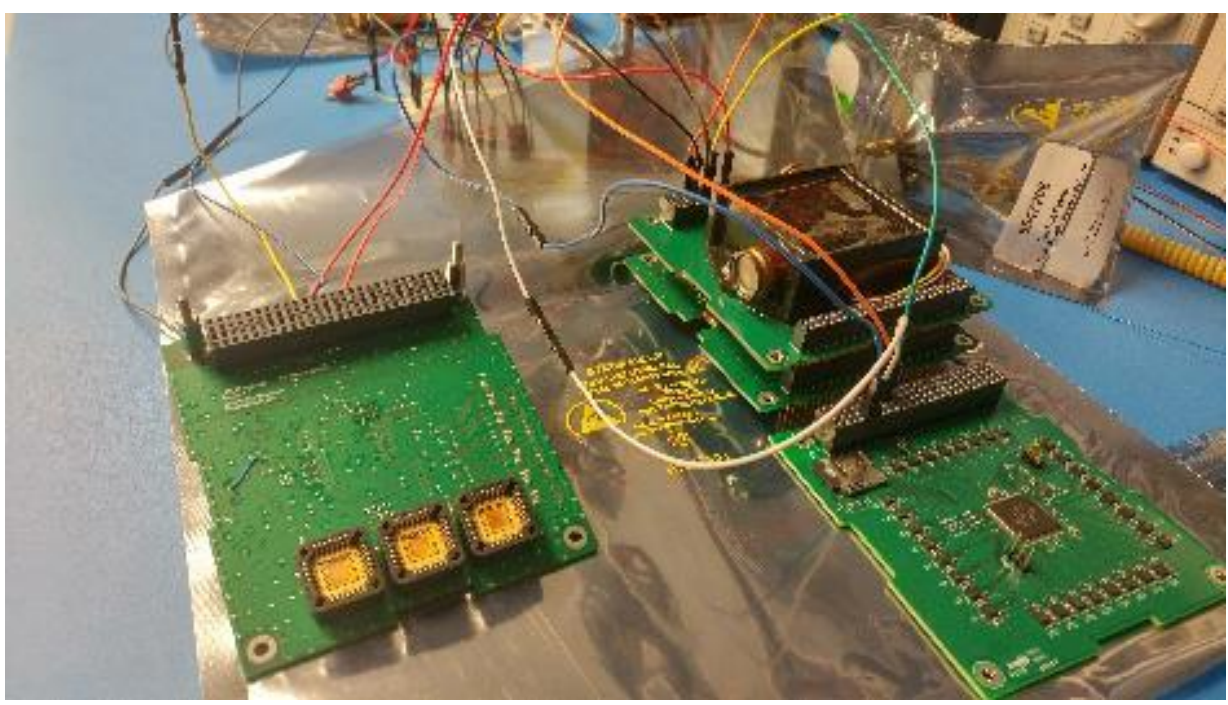

Figure 5. 5: I-V characterizer integration into CubeSat

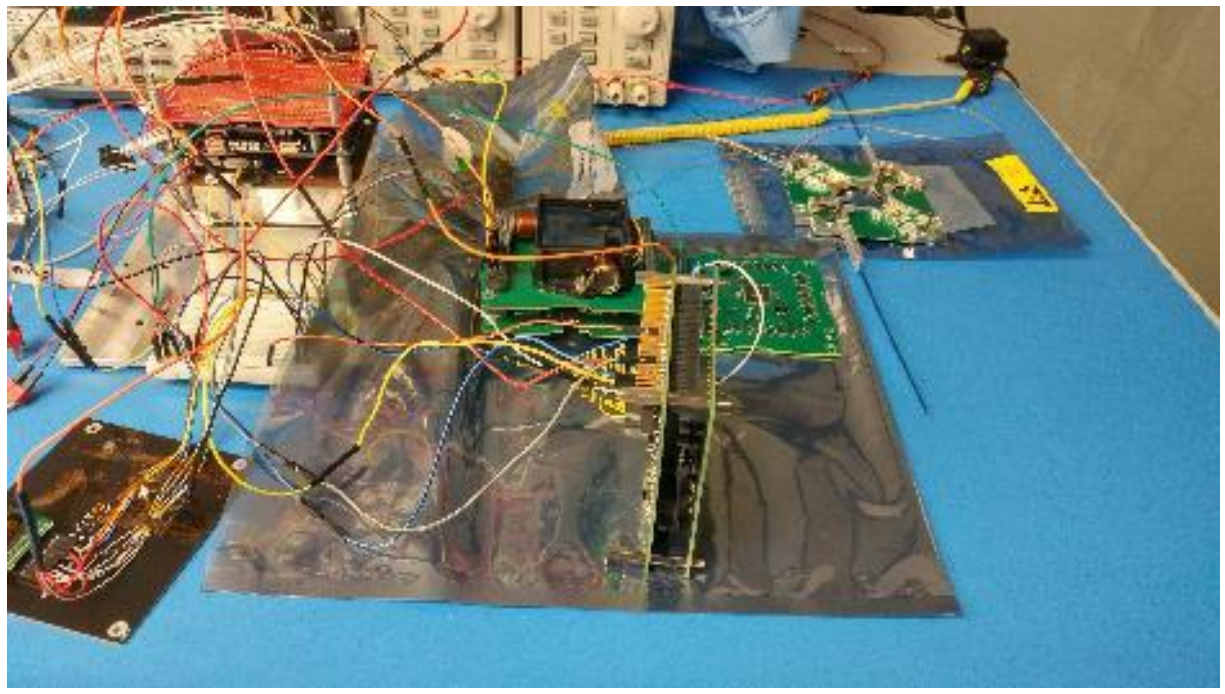

Figure 5. 6: Full LOCC integration into CubeSat

Figure 5.7 shows the final assembly of STF-1. This shows the complete system, ready for placement into the CubeSat launch vehicle payload. The outside casing is made up of four plates of solar cells that will recharge the internal battery for running experiments. On the top, a small antenna exists to communicate between the CubeSat and ground station for send and receiving the data. 


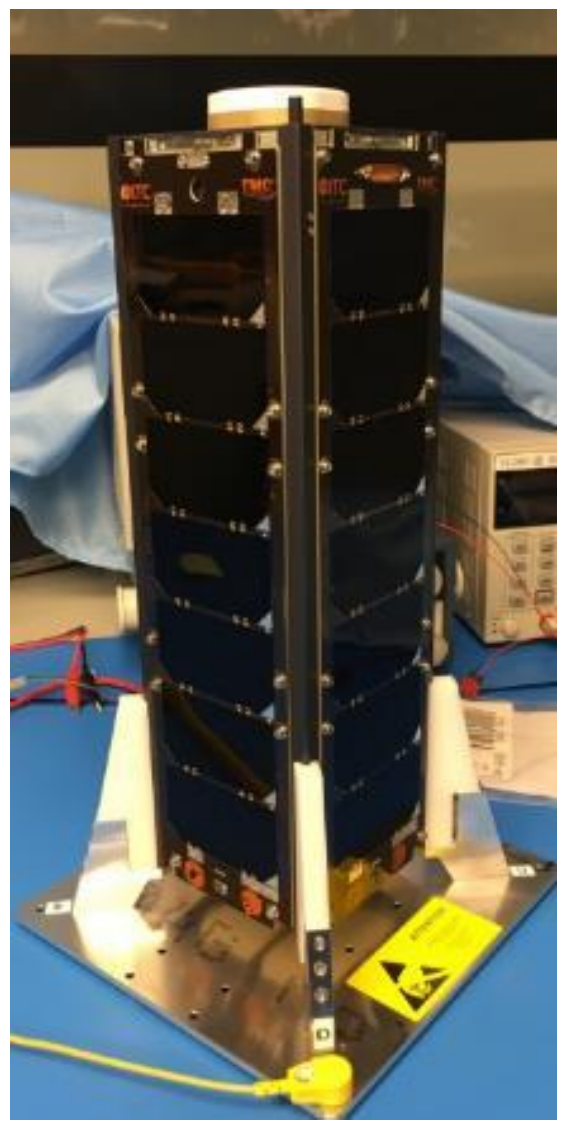

Figure 5. 7: Final assembly of the STF-1 CubeSat

\subsection{I-V Characterizer Testing}

The purpose of LOCC and its experiments is to provide a test for determining LED quality regarding their operation against irradiation. LOCC performs I-V characterization and EL estimations to provide information for examining voltage shifts, resistive changes, and peak wavelength shifts. The results of testing the LOCC I-V characterization module are examined below and consist of:

1) The resulting $I-V$ characteristics of each chip carrier set, along with a comparison of each LED over the course of 4 trial runs.

2) The mean and standard deviation of LED current and voltage between each chip carrier, as well as a mean and standard deviation of current and voltage for each LED. These 
results are examined to evaluate constancy between the LEDs over the course of 4 trials runs.

The output characteristics are shown in figures $5.8 \& 5.9$.

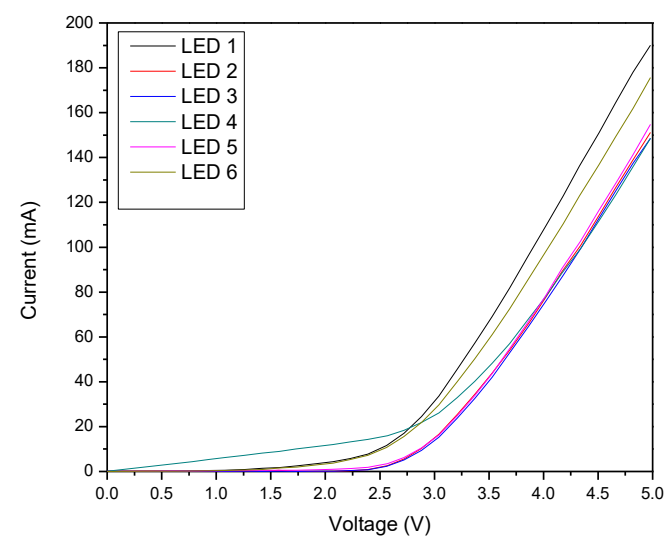

(a)

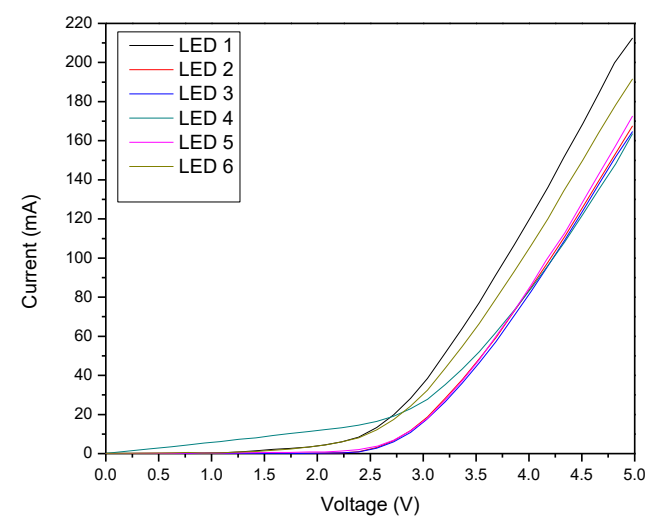

(c)

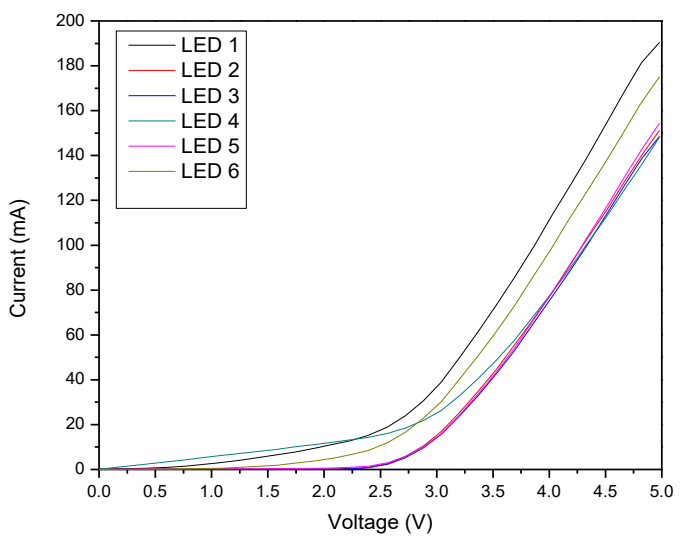

(b)

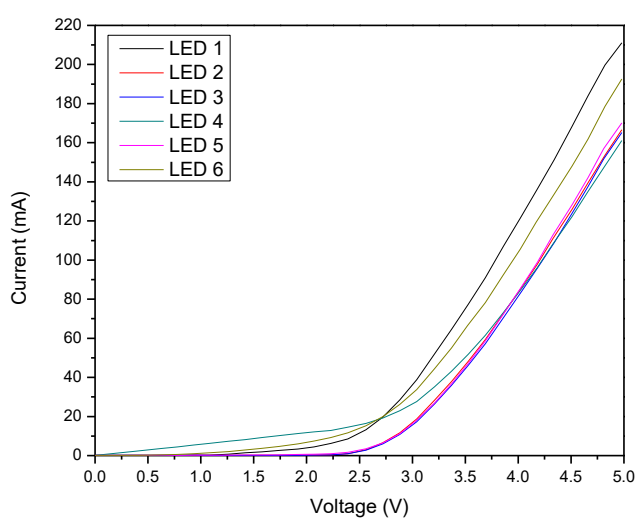

(d)

Figure 5. 8: I-V Characteristics of Chip Carrier 2. (a)Trial 1, (b)Trial 2, (c)Trial 3, (d) Trial 4 


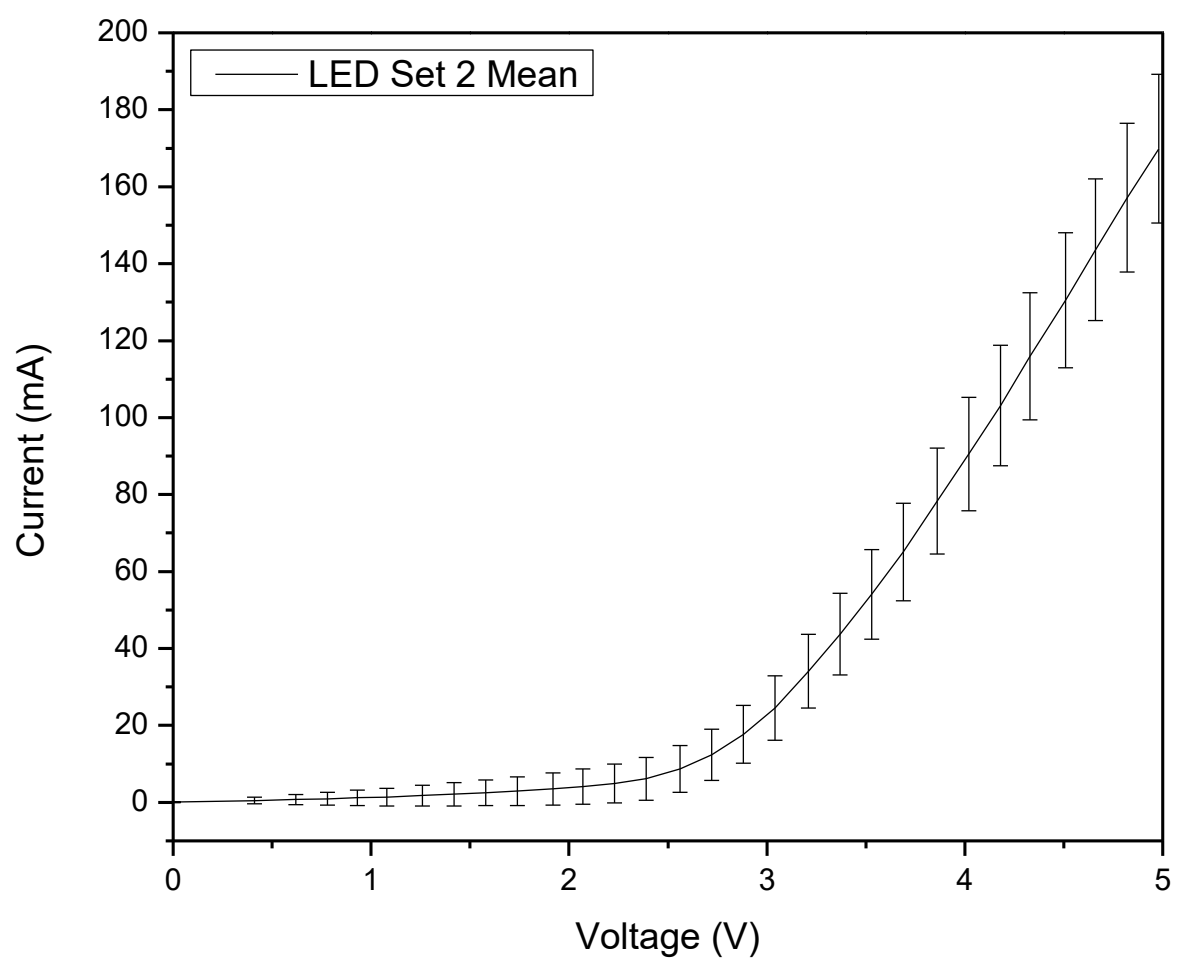

Figure 5. 9: Average and standard deviation I-V results of LEDs in chip carrier 2

Graphs 5.3.1 and 5.3.2 illustrate a successful characterization of chip carrier 2 LEDs. The LEDs that were characterized show turn-on voltages ranging from $1.6 \mathrm{~V}$ to $2.5 \mathrm{~V}$. A $36 \%$ difference is seen over the LED turn-on voltages for chip carrier 2. Early turn-on voltages can be seen in LEDs 1, 4, and 6 for the second trial. These LEDs show a change in turn-on voltages of $14.29 \%$ and $9.09 \%$, and 11.11\%, respectively, between trials 1 and 2, and back to trial 3. The remaining LEDs, showed little change, at less than $.80 \%$ in turn-on voltage values. The I-V characteristics also allow for the examination of the LED resistances. These resistance values vary $26 \%$ from the least resistive LED, LED 1 at $9.3 \Omega$, to the most resistive, LEDs 2, 3, and 5 of $12.5 \Omega$. LEDs 2, 3, and 5 show a resistive difference of $4 \%$, from $12 \Omega$ to $12.5 \Omega$. Examining figure 5.10 , the averaged results of the LEDs can be seen. This shows an average turn-on voltage of $2.46 \mathrm{~V}$, an average resistance of $11.65 \Omega$, and an 
average maximum current of $165 \mathrm{~mA}$. Figure 5.11 also shows gives information of the standard deviation from the mean value of the 4 trials. The standard deviation at $5 \mathrm{~V}$ is 20.5 and 8.7 at the mean turn-on voltage. While these parameters vary, they can be used during the process of gathering data. Temperature effects may have temporary effects that can be seen from experimental data over time, mean values of current, voltage, and resistance will have a have greater deviations over time and exposure to radiation. The standard deviations from the mean will also determine how each device is being affected, by temperature or radiation.

Following the examination of the LED chip carriers, individual LEDs are graphed and looked at for the same properties of current values, voltage shift, and standard deviation. Figures 5.12 shows three different LEDs ran over the course of 4 trails. Here, consistency in the LED measurements can be seen. This is shows turn-on voltage shifts of less than .2V, with LEDs 5, 6 , and 7 having .8\%, 1.3\%, and $4.4 \%$ differences in turn-on voltages, respectively. Resistance change was also minimal, with changes from $11.2 \Omega$ to $11.5 \Omega$ for $\operatorname{LED} 5,11.1 \Omega$ to $11.3 \Omega$ for LED 6 , and $11.5 \Omega$ to $11.6 \Omega$ for LED 7. Overall, this is less than a $2 \%$ difference across the LEDs. This further illustrates the consistent results provided by the LOCC I-V characterization system.

Figures 5.12 shows three different LEDs ran over the course of 4 trails. Standard deviations at $5 \mathrm{~V}$ were $20.1,17.5$, and 25.3 for LEDs 5,6 , and 7. 


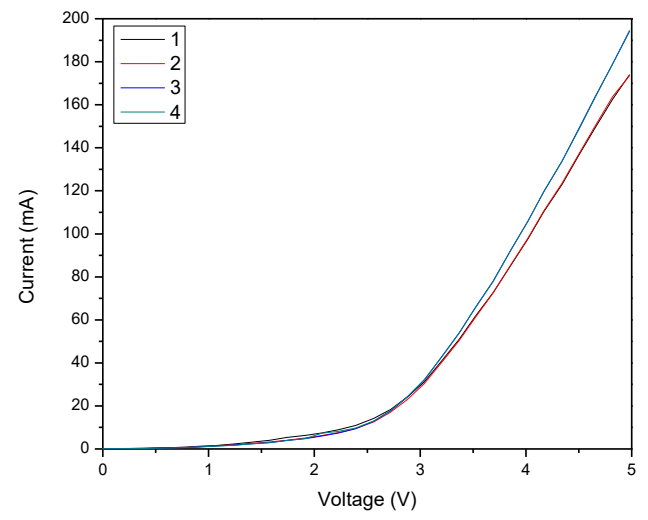

(a)

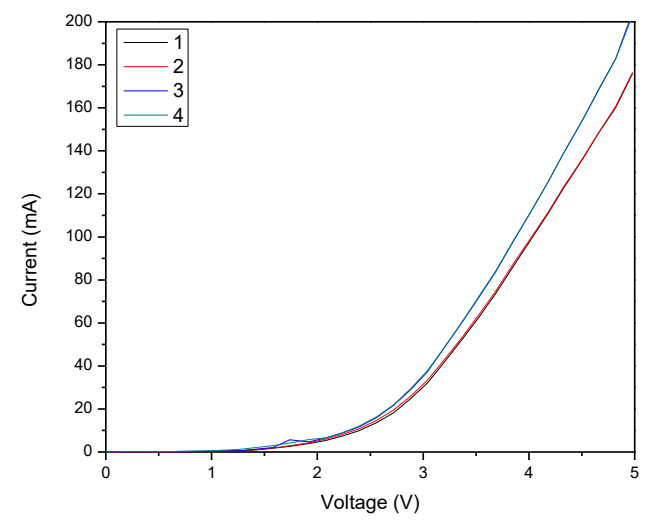

(c)

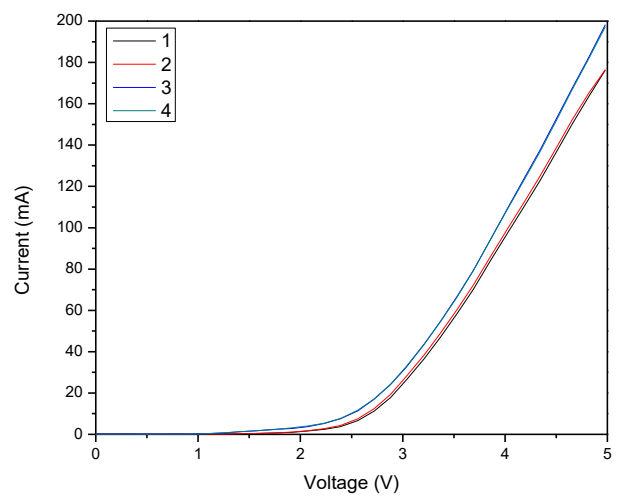

(e)

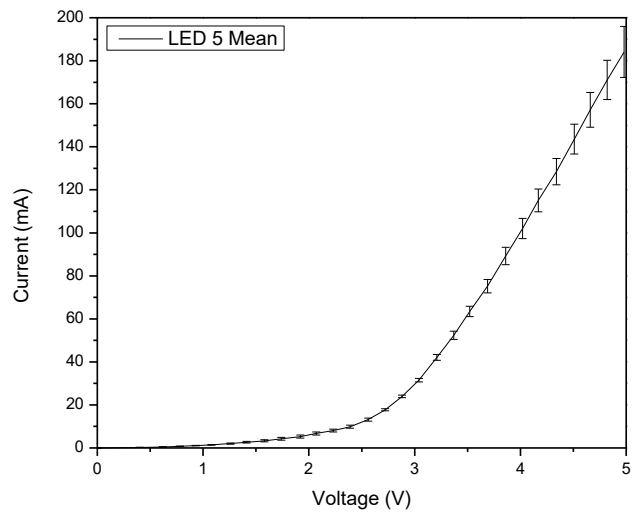

(b)

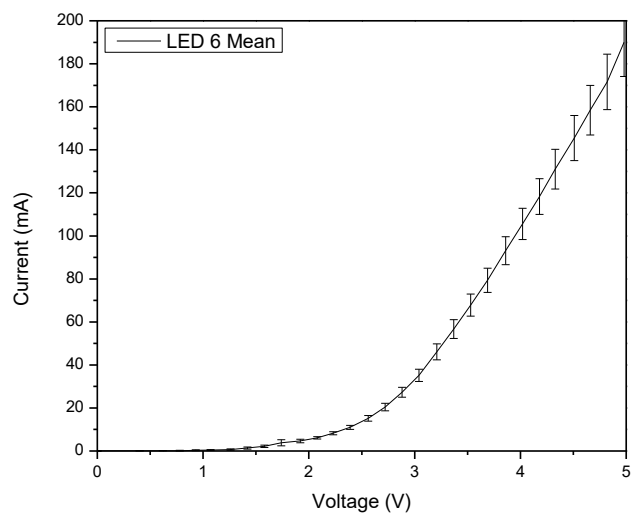

(d)

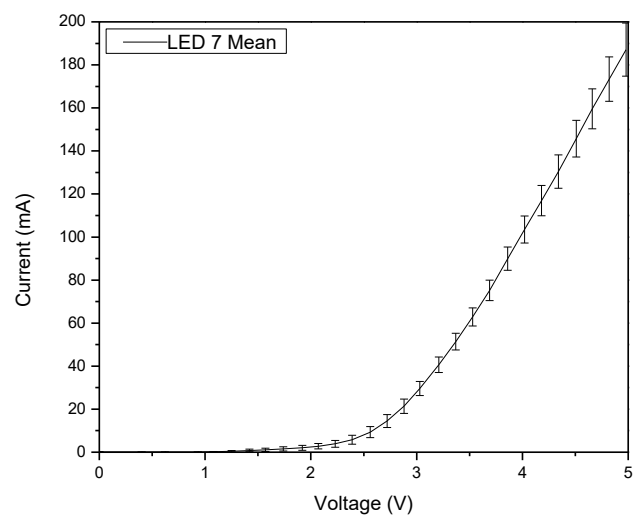

(f)

Figure 5. 10: (a)LED 5 - 4 trials, (b)LED 5 - Mean and standard deviation, (c)LED 6 - 4 trials, (d)LED 6 - Mean and standard deviation (e)LED 7 - 4 trials, (f)LED 7 - Mean and standard deviation 


\subsection{Electroluminescent Characterizer Results}

For the next portion of this section the electroluminescent characterizer results will be examined. As discussed before, the peak wavelength emission and light output of the LEDs are crucial measurements for determining the effects of radiation on the LEDs. The data from the trial runs aboard STF-1 are below. These results consist of:

1) The chromaticity diagram estimations of the peak wavelength emissions. Because the variance of these results are overlapping from a high-level view, the zoomed regions are included to highlight where the results lie in the diagram.

2) Secondly, the resulting light output values from the corrected xyY values are plotted of the functioning LEDs.

\subsubsection{Peak wavelength estimations}

Using MATLAB, the correlation matrix, described in section 2.3.2, is used against the recorded values from LOCC's EL characterizer. The results of the corrected values are then transformed into xycoordinates for the CIE 1931 color standard and plotted against the CIE 1931 chromaticity diagram. Figure 5.11 shows the plotted color corrected values for estimating peak wavelength.
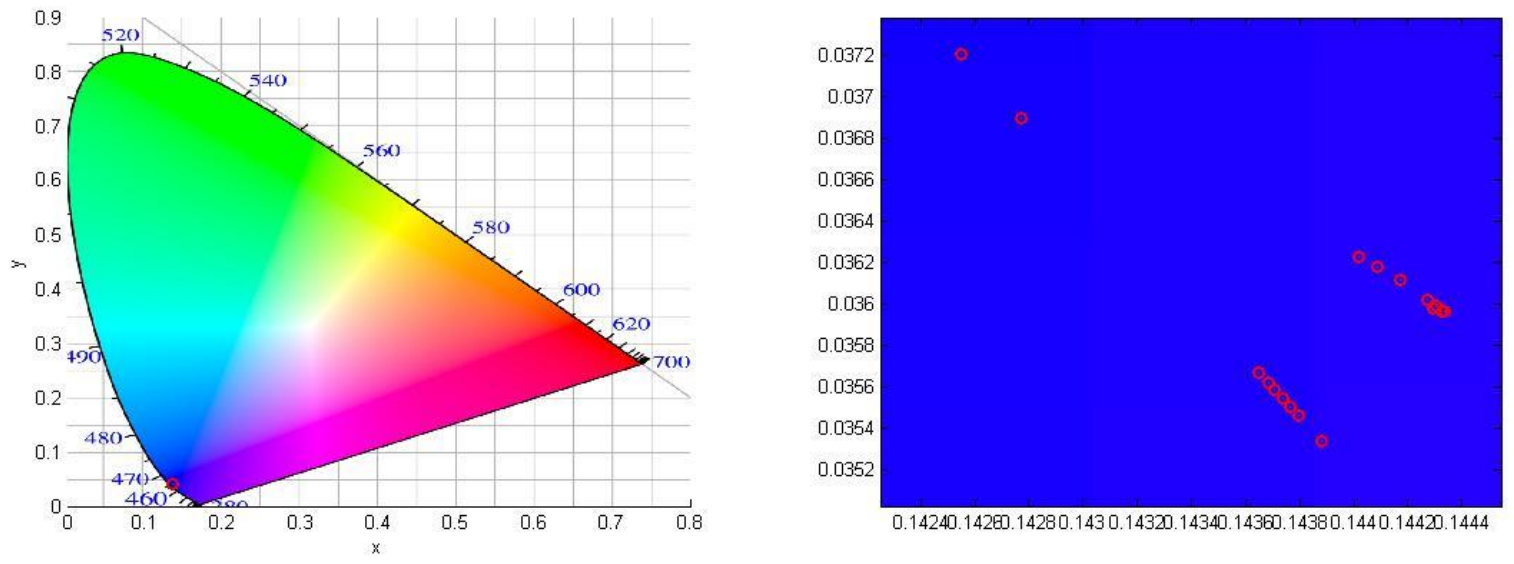
(a)

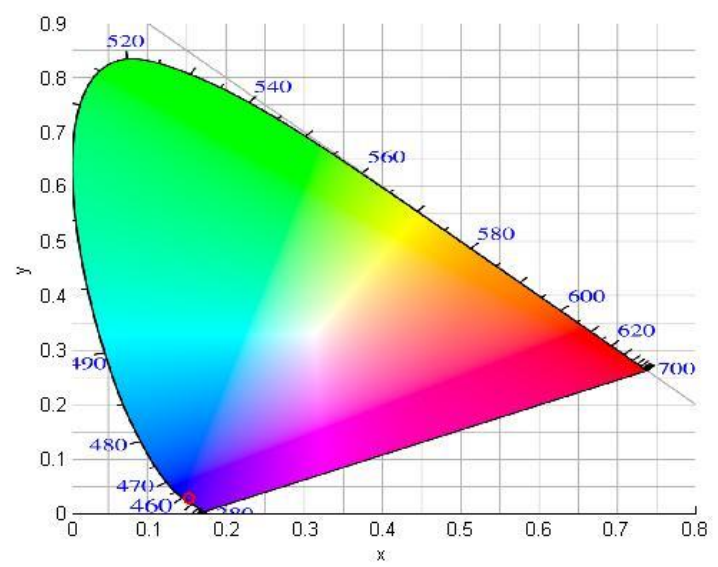

(c)

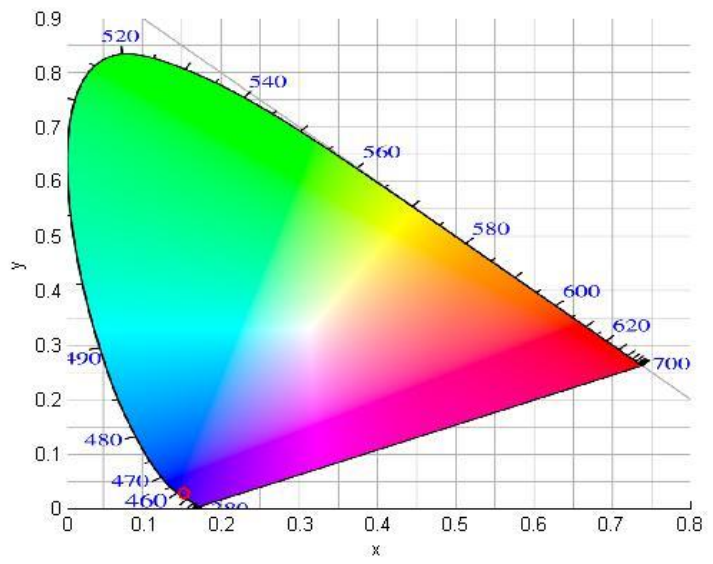

(e)

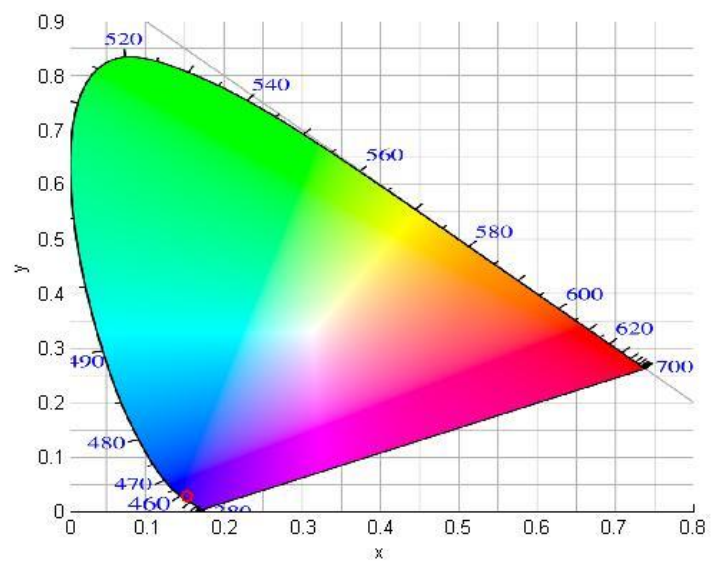

(g) (b)

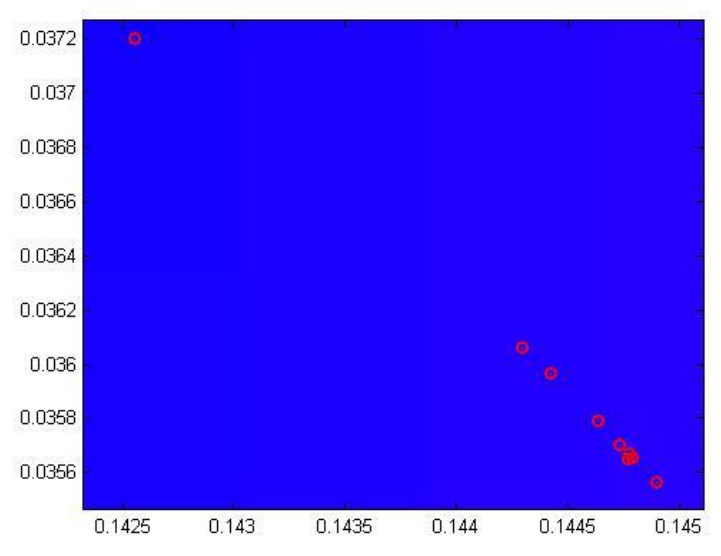

(d)

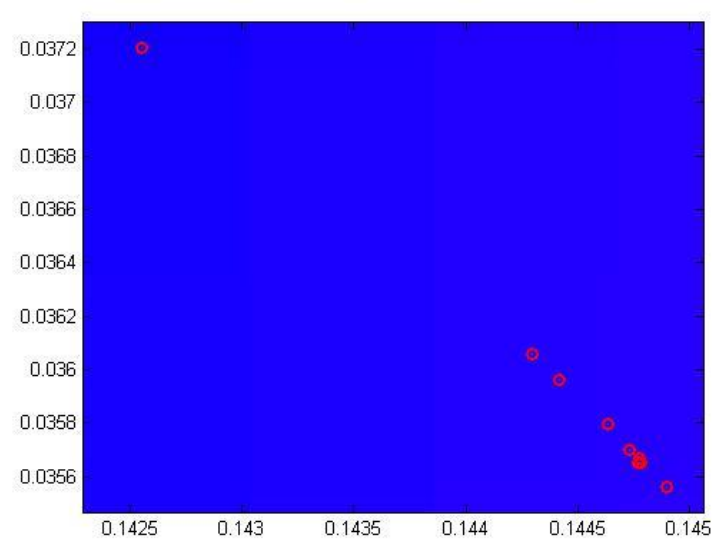

(f)

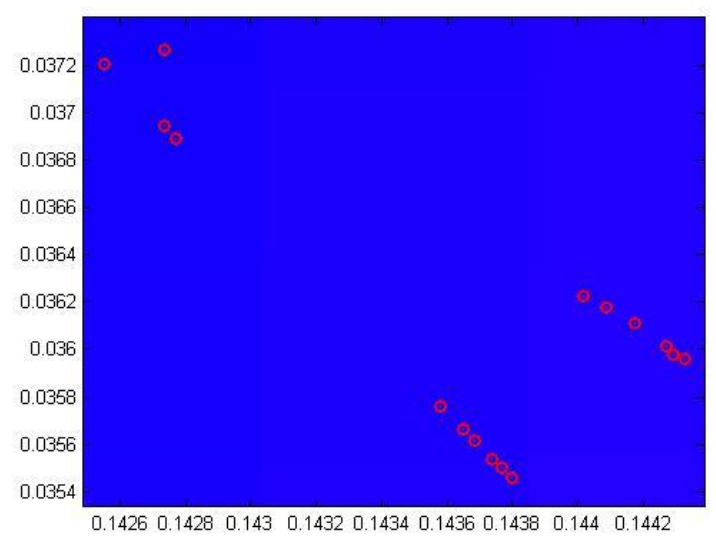

(h)

Figure 5. 11: EL Characteristics of all LEDs. (a)Trial 1 - 24 LEDs, (b)Trial 1 - Zoomed Chromaticity values, (c) Trial 2 - 24 LEDs, (d) Trial 2 - Zoomed Chromaticity values (e)Trial 3 - 24 LEDs, (f)Trial 3 Zoomed Chromaticity values, (g) Trial 4 - 24 LEDs 
The x-values range from .1426 to .1422 and y-values range from .0354 to .0372. The corrected values can be seen at the monochrome values between 455 and $460 \mathrm{~nm}$, which is consistent with the original EL measurements provided by the original in-house measurement system, seen in Figures 5.12. Because the correlated values are overlapping, additional plots zoomed around the values are provided below to see the difference in LED outputs.

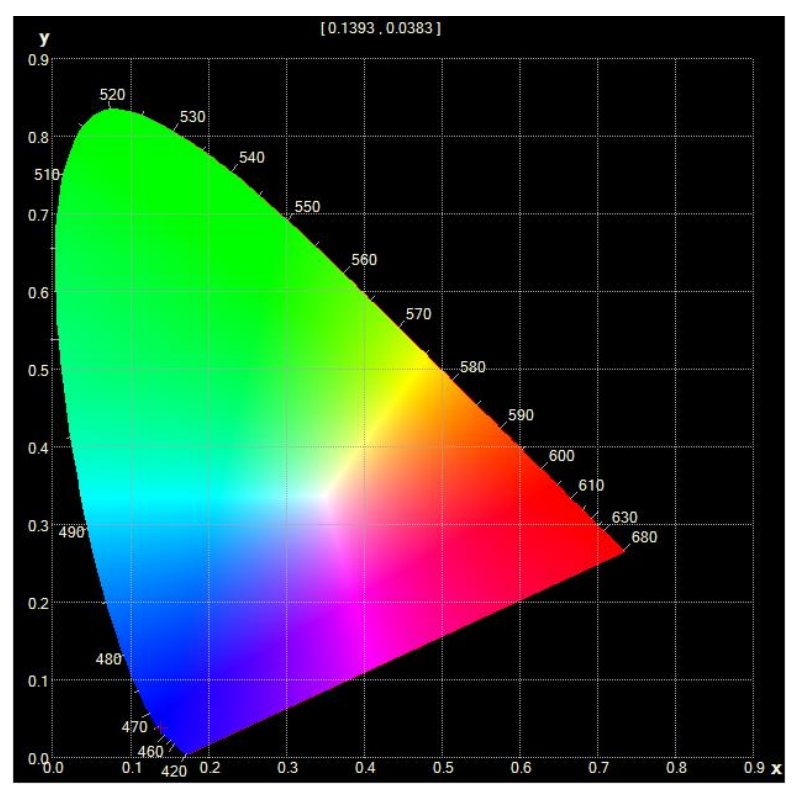

(a)

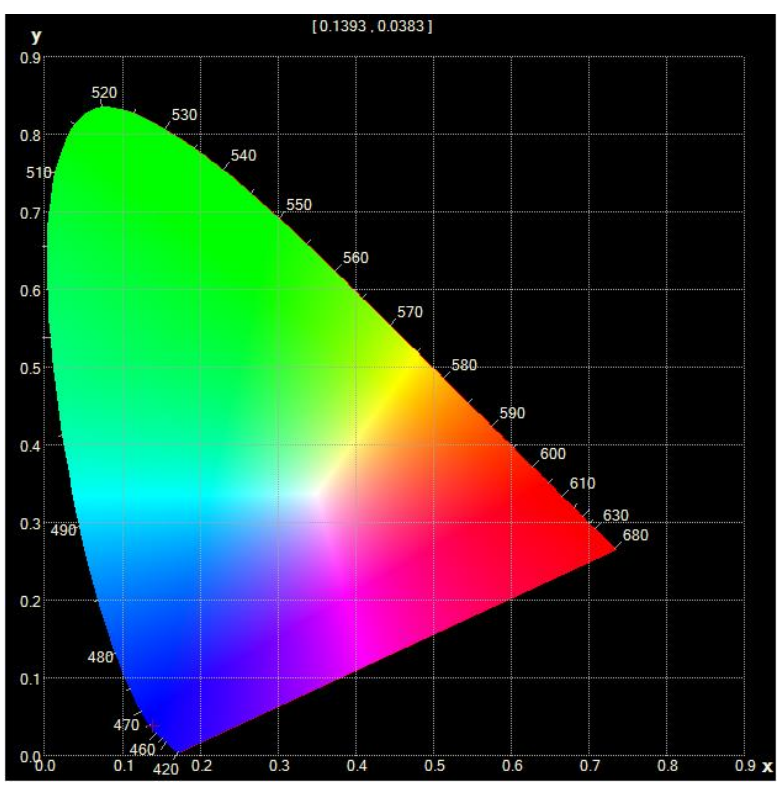

(b)

Figure 5. 12: Commercial Spectrometer Chromaticity outputs

\subsubsection{Light Output Results}

Figures $5.13-5.14$ describe the plotted light output intensity values as box diagrams to show where most of the calculated $\mathrm{Y}$ values fall in terms of light output intensity. Here, the $\mathrm{Y}$-values from the colorimetric reading hold the light output intensity values. These values are important as they can be used with Equation 2.26 to help describe the lifetime-damage coefficient. Here the values seen in 
the LEDs can range in difference from .12 to .2 in arbitrary units. These values are useful for determining lifetime-damage coefficients and determining light output degradation over time. Additionally, Figure 5.14 provides of overview of chip carrier 1 LED's light output intensity. As the effects of space takes place on the LEDs, from what research has shown us, these light output intensities will decrease overtime. The mean values of the trials are represented by the red bar inside the box, values within a standard deviation fall with the upper and lower limits of the box, and those connected with dotted lines are outside quartiles for light output values. Light output values range from .42 to .19 .

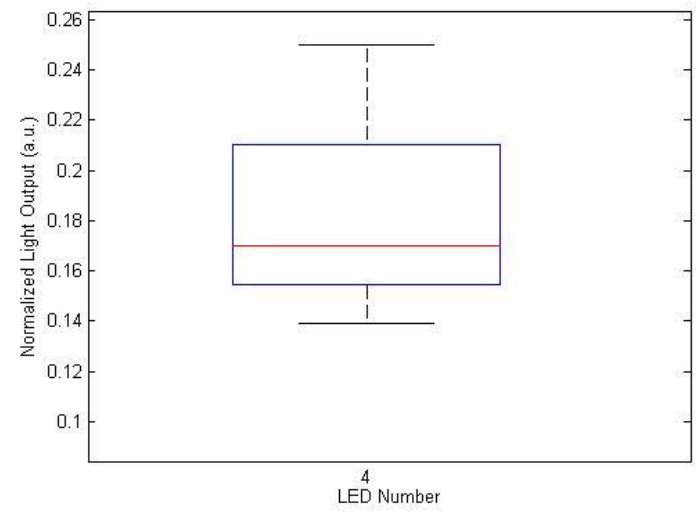

(a)

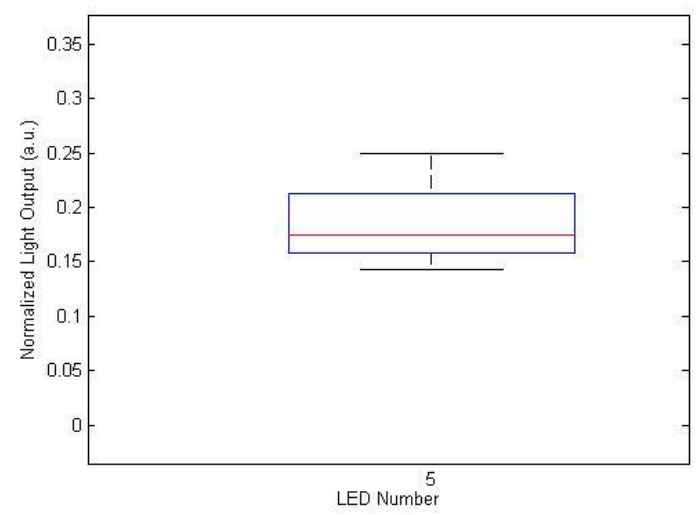

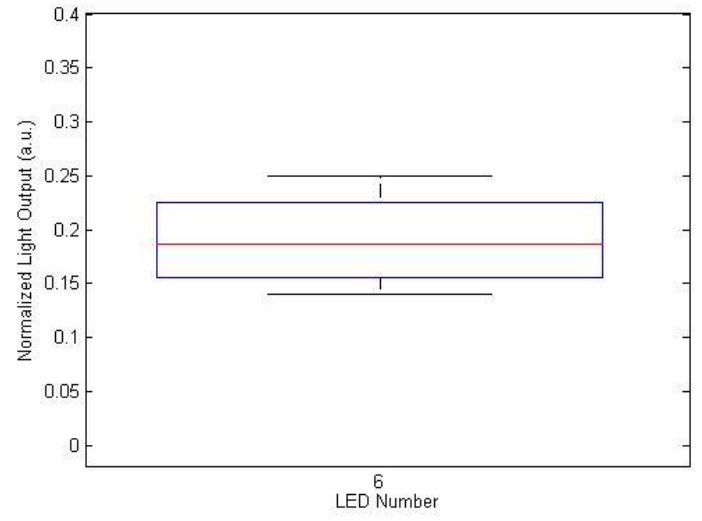

(b)

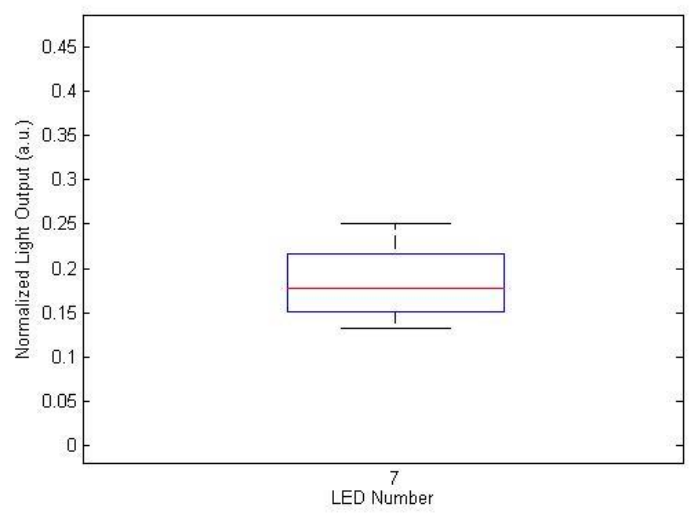


Figure 5. 13:Box plot of light output over 4 trials. (a) LED 4, (b) LED 5, (c) LED 6, (d) LED 7

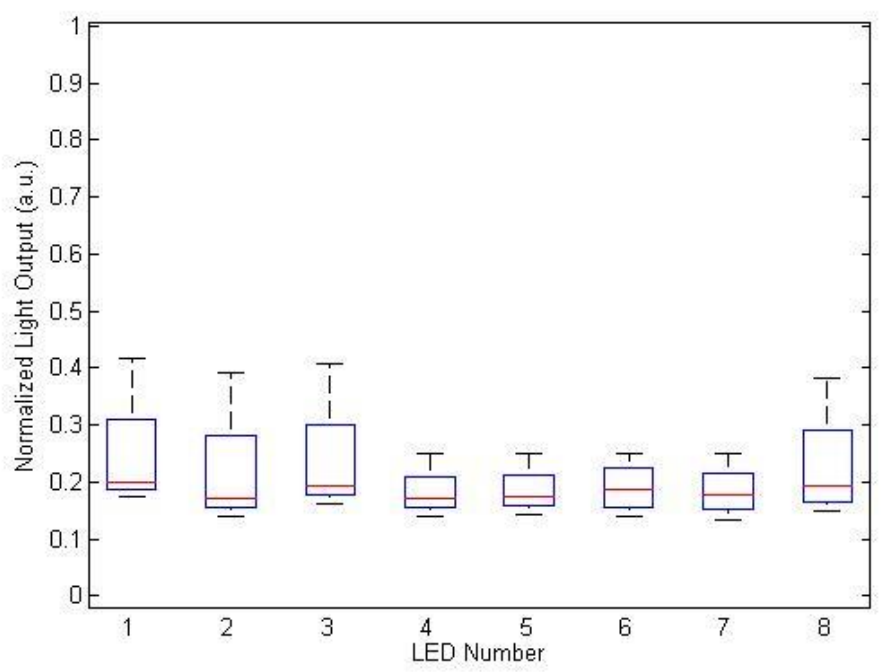

Figure 5. 14: Light output intensity of LED chip carrier 1

\subsection{Power and Data Budgets Results}

This subsection is dedicated to describing the power and data budgets of the LOCC system. This is broken down into the system's subsequent experiments, and results are plotted over the time. The plots below illustrate the current fluctuations observed while performing IV characterization, idle current consumption, and the max current consumption. Following this, the total required wattage and watt-hours are provided. The data requirements are more easily determined, as they take a fixed amount of memory per each experiment. 


\subsubsection{Power Results}

Before examining the power results, two considerations must be noted. First, because the STF1 flight computer continuously samples the output current and voltage of its batteries, the output currents do not directly correlate to the current requirements of each LED. This means that an LED, or even multiple LEDs, may have turned on in between the power points sampled by the flight computer. Secondly, the duration of each test must be noted. Experiment 1, which gathers data on the I-V measurements, takes $\sim 25$ seconds to complete. With the flight computer sampling every 10 seconds, this sampling rate is not fast enough to provide information related to current change over time. To overcome this challenge in power characterization, Experiment 1 was conducted multiple times for two trials. This was then reconstructed and presented over a 100 second span to match the duration of Experiment 2, which gathers the data from the EL measurements. Experiment 1 results are shown in Figure 5.15. These detail the current draw of the LOCC system when integrated into the CubeSat. Because the LEDs turn on an off one at a time, the overall current draw jumps from idle $.21 \mathrm{~A}$ to a maximum of $.72 \mathrm{~A}$ over the course of the experiment. Further, these the values of average maximum current, average current, and idle current are seen. The average maximum current draw is approximately .6A, average current draw over the duration of the experiment is $.432 \mathrm{~A}$, and idling is $.21 \mathrm{~A}$. 


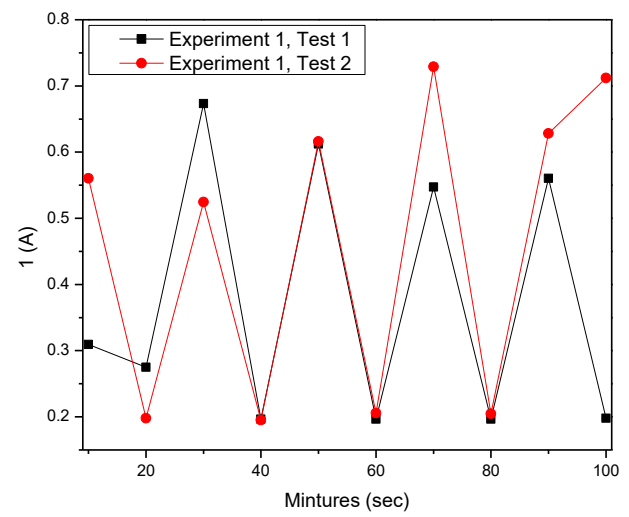

(a)

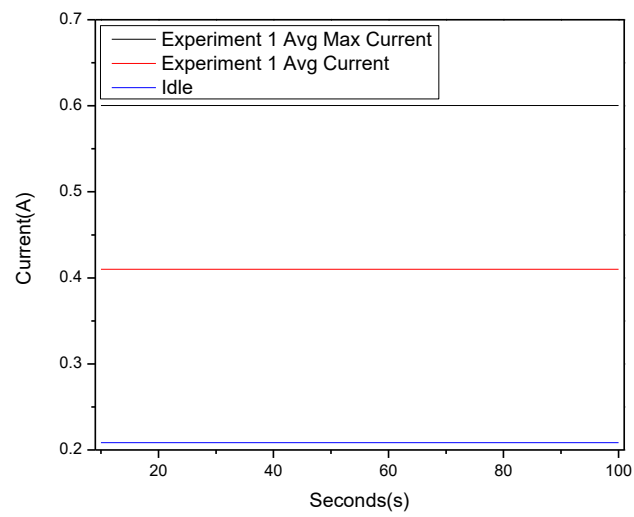

(b)

\section{Figure 5. 15: Experiment 1 (a) Current consumed over time, (b) Average current consumed over time}

The duration of Experiment 2 is much longer than the I-V characterization experiment because more steps are involved. Here, the experiment takes approximately 100 seconds to run through the entire set of 24 LEDs. The convention is the same as in Experiment 1, meaning the samples take place every 10 seconds and do not show the current change for every LED. Here, the values remain relatively the same with an increase in current draw by .02A due to the duration the LEDs are left on. The maximum current draw seen in the experiment is .7A up from the idle of .21A. Experiment 2's average maximum current draw is $.62 \mathrm{~A}$, average current draw is $.43 \mathrm{~A}$, and idle is still $.21 \mathrm{~A}$. 


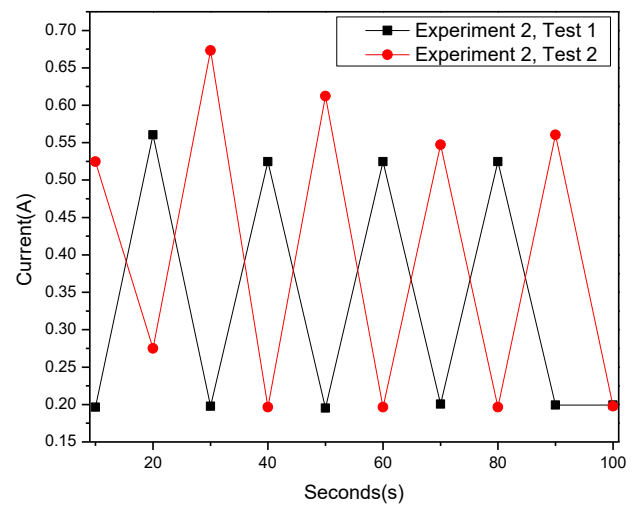

(a)

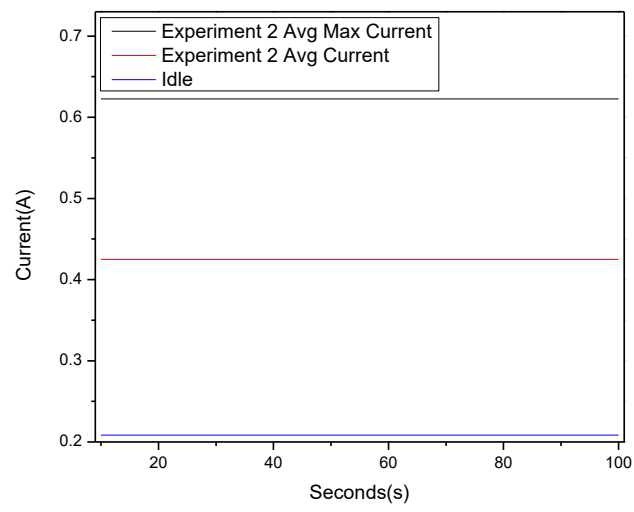

(b)

Figure 5. 16: Experiment 2 (a) Current consumed over time, (b) Average current consumed over time

Table 5.1 provides an overview of the current draw and power needed to run the LOCC system both idle and under load. The table gives an idea of the average current draw, maximum current draw, average wattage, maximum wattage, average watt-hours, and maximum watt-hours needed to deploy the LOCC system. This table represents the graphed values in the preceding figures. These values describe LOCCs necessary power requirements for integration into a CubeSat. The relatively low watthour demand provides a lower demand as it does not stay on for very long.

Table 5. 1: Power Results

\begin{tabular}{|c|c|c|c|c|c|c|}
\hline Experiment & $\begin{array}{c}\text { Avg } \\
\text { Current } \\
\text { (A) }\end{array}$ & $\begin{array}{c}\text { Max } \\
\text { Current } \\
\text { (A) }\end{array}$ & $\begin{array}{c}\text { Wattage } \\
\text { Avg (W) }\end{array}$ & $\begin{array}{c}\text { Wattage } \\
\text { Max (W) }\end{array}$ & $\begin{array}{c}\text { Watt-hour } \\
\text { Avg (Wh) }\end{array}$ & $\begin{array}{c}\text { Watt-hour } \\
\text { Max (Wh) }\end{array}$ \\
\hline I-V & 0.410 & 0.600 & 2.045 & 3.002 & .057 & .083 \\
\hline EL & 0.425 & 0.623 & 2.125 & 3.113 & .059 & .086 \\
\hline
\end{tabular}




\subsubsection{Data Budget Results}

The budget for data consists of the output files produced by the LOCC system. LOCC stores the measured values for LED current, voltage, and tristimulus results, and sends them to the flight computer for transmission to the ground station. These are initially stored as binary files, and must later be translated into text files. The resulting data budgets shown below are from both the generated binary and text files. Table 5.2 describes the data that is generated from the LOCC system. Initially, the user will receive a $4 \mathrm{kB}$ or $408 \mathrm{~B}$ binary file and have translated into a .txt which is now a $40 \mathrm{kB}$ or $4 \mathrm{kB}$ file. This shows that the data demand of LOCC is relatively low and can be accounted for in small storage space requirements.

\begin{tabular}{|c|c|c|}
\hline \multicolumn{3}{|c|}{ Table 5. 2: Data Budge Results } \\
\hline Experiment & .bin file size (B) & .txt file size (B) \\
\hline IV & 3096 & 39,500 \\
\hline EL & 408 & 3730 \\
\hline
\end{tabular}

\subsection{Summary of Results}

Using a combination of MATLAB, OriginPro 8, and custom Raspberry Pi software, I-V and EL characteristics for WVU's in-house fabricated III-V GaN LEDs have been produced. Using telemetry provided by STF-1's flight computer, the resulting power over time and energy needed to power the system were analyzed to show functionality and feasibility. Overall, the LOCC system is shown to be compatible for CubeSat applications, as well as being effective at measuring the optical and electrical characteristics of LEDs in the CubeSat platform. The LED samples were fabricated and 
packaged as three sets of $4 \times 4$ arrays, labeled as sets 1-3. To resolve reliability issues with the LOCC system, it is suggested that both commercial and custom LEDs be used to continually improve both fabrication and packaging steps of custom LEDs, and provide corrected and accurate results from the LOCC system.

The first portion of these results illustrate the electrical characteristics of the LEDs from 0$5 \mathrm{Vs}$ with increments of $.15 \mathrm{~V}$ allowing for 32 values of both current and voltage. This will allow the post-irradiated LEDs to be compared with the results produced from this testing with a second LOCC system (located on the ground) while this unit is in space. This will show if the system can detect electrical characteristic changes based on irradiation inside the ionosphere. For the second portion of this experiment, the CIE 1931 chromaticity values have been calculated from the results provided by LOCC. This requires additional steps to ensure that the correct values are found. This includes calculating a correlation matrix from known spectrometer values and applying them to the LOCC tristimulus values. From the results above, it is shown that the calculated values fall just below $460 \mathrm{~nm}$ wavelength emission, close to $455 \mathrm{~nm}$. This is consistent with characterization of the devices from the in-house equipment. Using the provided results and calculated correlation matrix, LOCC will be able to show if irradiation effects cause a shift in peak emission wavelength. The EL characterization results can also be used to determine the light output intensity. Because our most meaningful results will come from light intensity, it is important to state that these values are maintained at the corrected tristimulus values in the xyY coordinates. Here, LOCC will be able to detect changes in light output intensity values for irradiated devices, and allow for comparison against the pre-irradiated values on the ground station system for light intensity.

The last portion of the results consisted of the necessary budgets of the LOCC system. This included power and data of the system. The provided graphs are given as part of STF-1's housekeeping data to monitor the output of each pin on the battery system. The provided results have been averaged 
over the duration of the experiments and fit within a CubeSat's power budget. The small wattage required to run the experiments as well as watt-hours make LOCC ideal for scalability for larger experiments or multiple systems. Next, LOCC produced binary files to be translated into text files. Ultimately, LOCC's current configuration produced .bin files that do not exceed $4 \mathrm{~KB}$ of data, leaving room to produce larger files with greater accuracy and I-V steps.

Regarding costs, the largest cost of producing this system was from the custom-made LEDs. Another cost that can be adjusted for future work is the PCB production company. The initial version of the EL characterizer was produced locally and, due to their processing, made the price almost three times higher than having the PCB produced from a manufacturer geared towards high-volume productions. Potentially, the overall production cost of the LOCC can be reduced from $\$ 6500$ to almost $\$ 2000$, making this an academically feasible system to produce. In summary, the LOCC system shows potential as a system for characterizing LEDs effectively and cheaply while on orbit and provides a baseline system for introduction into the CubeSat and "NewSpace" industry. 


\section{Chapter 6: Conclusion and Future Work}

\subsection{Conclusion}

LEDs have revolutionized the lighting industry. The technologies and progress made in this industry have led to contributions in greater energy efficiency, fast methods of communication, and reliable devices for signal isolation. Along with this, optoelectronics have seen widespread application in the exploration of space and the "new space" industry as components of power generation, isolation, and communication systems. However, this does not mean that all optoelectronic materials and systems are immune to the effects of space such as proton and electron irradiation. Currently, to combat these effects, optoelectronics are heavily shielded, which adds to the overall cost and effectiveness of these devices.

Group III-V nitride materials are among the most popular materials being used in optoelectronic applications both, terrestrial and extraterrestrial, due to their material robustness in harsh environments. These robust properties are a by-product of the optimization of materials and methods used in the fabrication of the core elements of optoelectronic devices. When dealing with the effects of space, the overall characteristics of LED devices are the I-V characteristics, peak wavelength emission, and light output before and after irradiation for evaluating their performance in harsh conditions. Of these, the I-V characteristics and peak wavelength emissions have shown considerable reliability, but the optical characteristics (light output) experience more degradation before electrical degradation effects are observed. While examining LEDs under radiation bombardment, a method for examining the optical and electrical properties of LEDs while in space has been created.

A Low-power Optoelectronic Characterizer for CubeSats (LOCC) has been developed to place minimally shielded LEDs into orbit to perform experiments focused on characterizing both electrical and optical properties of $\mathrm{GaN}$ devices while in space. While many terrestrial experiments 
have been conducted on LEDs and other lighting devices haven't had LED characteristics quantified while in space. LOCC has been developed using a current-sourcing method to provide the necessary amount of current to drive the LEDs. LOCC is a statistical proof of concept to demonstrate the first field experiment of III-V Nitride based LEDs. The system is broken down into the current-voltage characterization module and electroluminescent characterization module. Furthermore, the system design utilizes power and communication isolation to protect itself and the bus from damaging effects to and from the CubeSat bus. Using low 'on' resistance switching and accurate current and voltage, as well as reliable photodetectors, LOCC has been implemented and tested within the CubeSat STF-1 to produce I-V characteristic diagrams, peak wavelength emission estimates, and light intensity measurements. Along with these results, low watt-hour requirements and data budgets have resulted in a scalable system capable of producing results within the CubeSat platform.

\subsection{Future Work}

LOCC utilizes an ATMEGA2560 microcontroller unit that is limited in memory capacity, therefore limiting the accuracy of the measurements. It is recommended that a MCU with higher memory capacity or an additional memory module be added to compensate for this. Another issue within the LED driving circuit is the lack of current limitation. This leaves some LEDs drawing far more current than the power supply is rated for as well as issues examining light output due to increase current draw for some LEDs. A current limiting resistor should be added to the driving circuit to limit draw to $\sim 100 \mathrm{~mA}$. While the EL characterizer can give an estimation of peak wavelength emission, it is recommended that this portion of the system undergo further development to utilize photosensors,

such as a CCD array that can scan the visible spectrum and recreate and accurate representation of the wavelength emission. Lastly, the cost to produce this prototype system can be reduced by selecting 
different vendors for PCB fabrication. Acquisition of an in-house soldering and reflow ovens should be considered to reduce cost of PCB assembly.

A major hurdle in the development and integration of this system was the packaging of the LEDs. The wire-bonding method employed in the packaging phase was under-developed, and led to a large variance in the characteristics of each LED. The caused the chip carrier bonding to suffer from short circuiting, fragile bonds, and limitations on the number of characterized LEDs. To save on future problems regarding LED connectivity, it is recommended to spend more time optimizing wirebonding techniques and pad creation to ensure proper bonding. Another consideration is to build a different layout that utilizes commercially available LEDs as they can provide a more reliable output and use them in parallel with custom LEDs. For future system development, it is recommended to use commercial LEDs to develop a baseline.

In Summary, a LED characterizer for CubeSats has been developed and integrated into West Virginia's first spacecraft, STF-1. The design and assembly of the LOCC system was performed simultaneously with the fabrication and packaging of the LEDs. With the help of members from NASA IV\&V, Ingeniu Tech, LLC, and the WVU Shared Cleanroom Facility, the LOCC system was made functional and successfully integrated. Significant improvements can be made to the system, but a working alpha-version system has been developed and can be improved upon to increase the scale of operation and reduce costs.

Future work includes:

- More in-depth simulations of controlling hardware components to increase accuracy, reduce power, and reduce data budgets - Simulation tools such as LABVIEW and Cadence PSpice are invaluable to producing simulated results that can more accurately reflect the output and data that will be acquired from a system. Continuing to refine the selected ICs and components of LOCC can provide for a far superior system for future renditions 
- Miniaturization of the characterization system to make feasible for desktop usage and control from personal computer - LOCC is made to be implemented into a CubeSat system and creates a tool for characterizing LEDs while in space These same concepts can be applied to making a similar system that is available for desktop usage and can be controlled via USB and software. Characterizing a bath of LEDs can takes hours, with a desktop version of the LOCC system it could be done in minutes.

- Testing of the LOCC system while under thermal stress and ionic bombardment to develop a more reliable system - To produce commercial grade electronics and systems they need to be subjected to rigorous testing that includes thermal cycling, vibration testing, military specification requirements for space, and so on. Creating LOCC and sending it through initial testing and qualifications to ensure a completely stable system

- Development and implementation of thermal management for both the I-V characterizer and EL characterizer - Because LOCC requires the use of optoelectronics and these components are sensitive to temperature flux, developing a thermally stable system will guarantee provided results remain as accurate as possible.

- Redesign of LOCC system to characterize optocouplers for improving design against radiation - Optocoupler are widely used in a multitude of electronics for separation of signal and avoiding ground loops. Many optocouplers are made to be radiation hardened and developing a system to characterize these devices while in space may prove beneficial for testing and device reliability.

- Development of CCD array electroluminescent characterizer for LOCC - LOCC utilizes an array of photodetectors that are dependent on CIE 1931 standard and human-eye perception of color. Conventional means of electroluminescent characterization utilize CCD arrays and wavelength sweeps to provide information of peak wavelength emission as well as the light 
bandwidth. LOCC and many other would benefit from design and development of CCD array EL characterizer for CubeSats to compliment the LOCC system.

- Further examination and simulation of the effects on LEDs while under radiation bombardment - Because we know that ion implantation is an effect of the space environment, utilizing simulation software such as SRIM and TRIM would provide insight for developing optoelectronic device structures that are radiation hardened.

- Simulation of passivation layers in the protection of LEDs against degradation in both electrical and optical properties - Materials such as Florine doped Tin-Oxide (FTO) and Indium doped Tin-Oxide (ITO) provide passivation layers to LEDs to increase their quantum efficiencies. Examining passivation layers as ionic stopping layers may be beneficial for reducing the effects of ion implantation into the active layers of optoelectronic structures. 


\section{References}

[1] Kulu, E. (2017). Nanosatellites \& CubeSat Database. Retrieved March 17, 2017, from http://www.nanosats.eu/

[2] Prince, F. A., "Weight and the Future of Space Flight Hardware Cost Modeling," 2003.

[3] Mosher, S. K. (2016, July 20). Here's how much money it actually costs to launch stuff into

space. Retrieved March 17, 2017, from http://www.businessinsider.com/spacex-rocket-cargoprice-by-weight-2016-6/

[4] Kestenbaum, D. (2011, July 21). Spaceflight Is Getting Cheaper. But It's Still Not Cheap Enough. Retrieved March 17, 2017, from

http://www.npr.org/sections/money/2011/07/21/138166072/spaceflight-is-getting-cheaper-butits-still-not-cheap-enough

[5] Marshall, P. W., Dale, C. J., and Burke, E. A., "Space radiation effects on optoelectronic materials and components for a $1300 \mathrm{~nm}$ fiber optic data bus," IEEE transactions on nuclear science, vol. 39, 1992, pp. 1982-1989.

[6] Johnston, A. H., "Radiation damage of electronic and optoelectronic devices in space," Proceedings of the 4th International Workshop on Radiation Effects on Semiconductor Devices for Space Application, 2000.

[7] California Polytechnic State University, "CubeSat Design Specification (CDS).”

[8] Daniel, L Oltrogge, and Kyle Leveque, “An Evaluation of CubeSat Orbital Decay,” 25th Annual AIAA/USU Conference on Small Satellites, Aug. 2012.

[9] Kadiyala, A., Kyoungnae L., Rodak L. E., Lawrence L. A., Korakakis D., and Dawson J.M. "Improvement in the Light Extraction of Blue InGaN/GaN-Based LEDs Using Patterned Metal Contacts.” IEEE Journal of the Electron Devices Society 2, no. 2 (March 2014): 16-22. doi:10.1109/JEDS.2013.2289308

[10] Justice, J., A. Kadiyala, Dawson, M.J., and Korakakis, D., "Group III-Nitride Based Electronic and Optoelectronic Integrated Circuits for Smart Lighting Applications." Materials Research Society Symposium Proceedings 1492, vol. 12 (2012): 123-28. doi:10.1557/opl.2013.369.

[11] Kadiyala, A., Kyoungnae L.,Rodak L.E., Hornak L. A., Korakakis, D. and Dawson, J. M., "Improvement in the Light Extraction of Blue InGaN/GaN-Based LEDs Using Patterned Metal Contacts.” IEEE Journal of the Electron Devices Society 2, no. 2 (March 2014): 16-22. doi:10.1109/JEDS.2013.2289308. 
[12] Zeynali, O., Masti, D., and Gandomkar, S., "Shielding protection of electronic circuits against radiation effects of space high energy particles," Advances in Applied Science Research, vol. 3, 2012, pp. 446-451.

[13] Zeynali, O., Masti, D., Ebrahimi, A., Orvatinia, M., and Maryam, N., “The design and simulation of the shield reduce ionizing radiation effects on electronic circuits in satellites," Electrical and Electronic Engineering, vol. 1, 2011, pp. 112-116.

[14] Maurer, R. H., Fraeman, M. E., Martin, M. N., and Roth, D. R., "Harsh Environments: Space Radiation," Johns Hopkins APL technical digest, vol. 28, 2008, p. 17.

[15] Robbins, M. S., "High-energy proton-induced dark signal in silicon charge coupled devices," IEEE transactions on Nuclear Science, vol. 47, 2000, pp. 2473-2479 [16] Cai, S. J., Tang, Y. S., Li, R., Wei, Y. Y., Wong, L., Chen, Y. L., Wang, K. L., Chen, M., Schrimpf, R. D., Keay, J. C., and others, “Annealing behavior of a proton irradiated Al/sub $\mathrm{x} / \mathrm{Ga} /$ sub 1-x/N/GaN high electron mobility transistor grown by MBE," IEEE Transactions on Electron Devices, vol. 47, 2000, pp. 304-307.

[17] Luo, B., Johnson, J. W., Ren, F., Allums, K. K., Abernathy, C. R., Pearton, S. J., Dwivedi, R., Fogarty, T. N., Wilkins, R., Dabiran, A. M., and others, "DC and RF performance of protonirradiated AlGaN/GaN high electron mobility transistors,” Applied Physics Letters, vol. 79, 2001, pp. 2196-2198.

[18] White, B. D., Bataiev, M., Brillson, L. J., Choi, B. K., Fleetwood, D. M., Schrimpf, R. D., Pantelides, S. T., Dettmer, R. W., Schaff, W. J., Champlain, J. G., and Mishra, U. K., "Characterization of 1.8-MeV proton-irradiated AlGaN/GaN field-effect transistor structures by nanoscale depth-resolved luminescence spectroscopy," IEEE Transactions on Nuclear Science, vol. 49, Dec. 2002, pp. 2695-2701.

[19] K. Gill et al., J. Appl. Phys. vol. 82, no. 1, July 1997.

[20] G.P. Summers et al., IEEE Trans. Nucl. Sci. vol. 34, 1987, p. 1134.

[21] Casse, G., "Radiation Damage in Silicon," Radiation Effects in Semiconductors, CRC Press, 2010, pp. 3-30.

[22] Lindström, G., Ahmed, M., Albergo, S., Allport, P., Anderson, D., Andricek, L., Angarano, M. M., Augelli, V., Bacchetta, N., Bartalini, P., and others, "Radiation hard silicon detectorsdevelopments by the RD48 (ROSE) collaboration," Nuclear Instruments and Methods in Physics Research Section A: Accelerators, Spectrometers, Detectors and Associated Equipment, vol. 466, 2001, pp. 308-326. 
[23] Dittongo, S., Bosisio, L., Ciacchi, M., Contarato, D., D’Auria, G., Fretwurst, E., and Lindstrom, G., "High-energy electron irradiation of different silicon materials," IEEE Transactions on Nuclear Science, vol. 51, Oct. 2004, pp. 2794-2798.

[24] Spieler, H., Lumpkin, A., and Eyeberger, C. E., "Introduction to radiation-resistant semiconductor devices and circuits," AIP Conference Proceedings, AIP, 1997, pp. 23-49.

[25] Patterson, R. L., Hammoud, A., and Elbuluk, M., "Electronic components for use in extreme temperature aerospace applications," 2008.

[26] L. Polenta, Z-Q. Fang, and D. C. LookView Affiliations DOI:

http://dx.doi.org/10.1063/1.126263, “On the main irradiation-induced defect in GaN,”Superradiation hard detector technologies: 3-D and widegap detectors. [27] Xinwen Hu, Karmarkar, A. P., Bongim Jun, Fleetwood, D. M., Schrimpf, R. D., Geil, R. D., Weller, R. A., White, B. D., Bataiev, M., Brillson, L. J., and Mishra, U. K., "Proton-irradiation effects on AlGaN/AlN/GaN high electron mobility transistors," IEEE Transactions on Nuclear Science, vol. 50, Dec. 2003, pp. 1791-1796.

[28] Ionascut-Nedelcescu, A., Carlone, C., Houdayer, A., von Bardeleben, H. J., Cantin, J.-L., and Raymond, S., "Radiation hardness of gallium nitride," IEEE Transactions on Nuclear Science, vol. 49, Dec. 2002, pp. 2733-2738.

[29] Belyaev, A. E., Boltovets, N. S., Konakova, R. V., Milenin, V. V., Sveshnikov, Y. N., and Sheremet, V. N., "Radiation damage of contact structures with diffusion barriers exposed to irradiation with 60Coy-ray photons," Semiconductors, vol. 44, Apr. 2010, pp. 448-456.

[30] S. J. Pearton, Y.- S. Hwang, and F. Ren, J. Mater. 67, 1601 (2015).

[31] M. G. Ganchenkova and R. M. Nieminen, Phys. Rev. Lett., 96, 196402 (2006).

[32] S. J. Pearton, R. Deist, F. Ren, L. Liu, A. Y. Polyakov, and J. Kim, J. Vac. Sci. Technol. A 31, 050801 (2013).

[33] M. R. Hogsed, Y. K. Yeo, M. Ahoujja, M.-Y. Ryu, J. C. Petrosky, and R. L. Hengehold, Appl. Phys. Lett. 86, 261906 (2005).

[34] S. A. Vitusevich, N. Klein, A. E. Belyaev, S. V. Danylyuk, M. V. Petrychuk, R. V. Konakova, A. M. Kurakin, A. E. Rengevich, A. Yu. Avksentyev, B. A. Danilchenko, V. Tilak, J. Smart, A. Vertiatchikh, and L. F. Eastman, Phys. Stat. Solidi A 195, 101 (2003).

[35] G. A. Umana-Membreno, J. M. Dell, G. Parish, B. D. Nener, L. Faraone, and U. K. Mishra, IEEE Trans. Electron. Dev. 50, 2326 (2003). 
[36] G. A. Umana-Membreno, J. M. Dell, G. Parish, B. D. Nener, L. Faraone, S. Keller, and U. K. Mishra, J. Appl. Phys., 101, 054511 (2007).

[37] J. T. Moran, J. W. McClory, J. C. Petrosky, and G. C. Farlow, IEEE Trans. Nucl. Sci., 56, 3223 (2009).

[38] B. H. Rose and C. E. Barnes, J. Appl. Phys. 53, 1772 (1982)., .-Y. Kim, J. Kim, F. Ren, and S. Jang, J. Vac. Sci. Technol B, 28, 27 (2010)., C. Liu, Optics and Spectroscopy 113, 153 (2012).

[39] M. Osi'nski, P. Perlin, H. Sch“one, A. H. Paxton, and E. W. Taylor, Electron. Lett. 33, 1252 (1997).

[40] S. M. Khanna, D. Estan, A. Houdayer, H. C. Liu, and R. Dudek, IEEE Trans Nucl. Sci. 51, 3585 (2004).

[41] S. M. Khanna, D. Estan, L. S. Erhardt, A. Houdayer, C. Carlone, A. Ionascut-Nedelcescu, S. R. Messenger, R. J. Walters, G. P. Summers, J. H. Warner, and I. Jun, IEEE Trans. Nucl. Sci. 51, 2729 (2004).

[42] B.-J. Kim, Y. H. Hwang, S. Ahn, F. Ren, S. J. Pearton, and J. Kim, J. Vac. Sci. Technol. B 33, 051215 (2015).

[43] A. Ionascut-Nedelcescu, C. Carlone, A. Houdayer, H. J. von Bardeleben, J.-L. Cantin, and S. Raymond, IEEE Trans. Nucl. Sci., 49, 2733 (2002).

[44] C. S. Lee and S. Subramanian, IEEE Trans Nucl. Sci. 50, 1998 (2003).

[45] Hong-Yeol Kim; Jihyun Kim, "Effect of neutron irradiation on electrical and optical properties of InGaN/GaN light-emitting diodes," Soohwan Jang; Journal of Vacuum Science \& Technology B, Nanotechnology and Microelectronics: Materials, Processing, Measurement, and Phenomena 2010, 28, 27-29. DOI: 10.1116/1.3268136

[46] C. Liu, Optics and Spectroscopy 113, 153 (2012).

[47] G. A. Umana-Membreno, J. M. Dell, T. P. Hessler, B. D. Nener, G. Parish, L. Faraone, and U. K. Mishra, Appl. Phys. Lett. 80, 4354 (2002).

[48] J. Tringe, A. Conway, T. Felter, W. Chan, J. Castelaz, V. Lordi, Y. Xia, C. Stevens, and C. Wetzel, Nuclear Science, IEEE Transactions on, 55, 3633 (2008).

[49] Champion, C. L., and La Rue, G. S., “Accurate SPICE models for CMOS analog radiationhardness-by-design," IEEE Transactions on Nuclear Science, vol. 52, Dec. 2005, pp. 2542-2549. [50] McIver, J. K., and Clark, L. T., "Reducing radiation-hardened DigitalCircuit power consumption," IEEE Transactions on Nuclear Science, vol. 52, Dec. 2005, pp. 2503-2509. 
[51] Kauppila, J. S., Massengill, L. W., Holman, W. T., Kauppila, A. V., and Sanathanamurthy, S., "Single event Simulation methodology for analog/mixed signal design hardening," IEEE

Transactions on Nuclear Science, vol. 51, Dec. 2004, pp. 3603-3608.

[52] Knudsen, J. E., and Clark, L. T., “An Area and Power Efficient Radiation Hardened by Design Flip-Flop,” IEEE Transactions on Nuclear Science, vol. 53, Dec. 2006, pp. 3392-3399.

[53] Chen, T.-H., Chen, J., Clark, L. T., Knudsen, J. E., and Samson, G., “Ultra-Low Power Radiation Hardened by Design Memory Circuits," IEEE Transactions on Nuclear Science, vol. 54, Dec. 2007, pp. 2004-2011.

[54] Chen, D., Kim, H., Phan, A., Wilcox, E., LaBel, K., Buchner, S., Khachatrian, A., and Roche, N., "Single-Event Effect Performance of a Commercial Embedded ReRAM," IEEE Transactions on Nuclear Science, vol. 61, Dec. 2014, pp. 3088-3094.

[55] Balasubramanian, A., Bhuva, B. L., Black, J. D., and Massengill, L. W., "RHBD techniques for mitigating effects of single-event hits using guard-gates," IEEE Transactions on Nuclear Science, vol. 52, Dec. 2005, pp. 2531-2535.

[54] Rhouni, A., Techer, J.-D., Sou, G., and Berthomier, M., "Design and Characterization of a High Dynamic Range and Ultra Low Power 16-Channel ASIC for an Innovative 3D Imaging Space Plasma Analyzer,” IEEE Transactions on Nuclear Science, vol. 59, Oct. 2012, pp. 2580-2586. [55] Cabanas-Holmen, M., Cannon, E. H., Amort, T., Ballast, J., Brees, R., Fischer, S., Kleinosowski, A. J., Meaker, B., Swann, T., and Wert, J., "Predicting the Single-Event Error Rate of a Radiation Hardened by Design Microprocessor," IEEE Transactions on Nuclear Science, vol. 58, Dec. 2011, pp. 2726-2733.

[56] Pickel, J. C., Reed, R. A., Ladbury, R., Rauscher, B., Marshall, P. W., Jordan, T. M., Fodness, B., and Gee, G., "Radiation-induced charge collection in infrared detector arrays," IEEE Transactions on Nuclear Science, vol. 49, Dec. 2002, pp. 2822-2829.

[57] Marshall, P. W., Dale, C. J., Burke, E. A., Summers, G. P., and Bender, G. E., "Displacement damage extremes in silicon depletion regions," IEEE Transactions on Nuclear Science, vol. 36, 1989, pp. 1831-1839.

[58] Hopkinson, G. R., “Radiation effects in a CMOS active pixel sensor,” IEEE Transactions on Nuclear Science, vol. 47, 2000, pp. 2480-2484.

[59] Bogaerts, J., Dierickx, B., and Mertens, R., "Enhanced dark current generation in protonirradiated CMOS active pixel sensors," IEEE Transactions on nuclear science, vol. 49, 2002, pp. 1513-1521. 
[60] Round, H. J. (1907). "A note on carborundum". Electrical World. 19: 309

[61] Holonyak Nick; Bevacqua, S. F. (December 1962). "Coherent (Visible) Light Emission from Ga(As1-x Px) Junctions". Applied Physics Letters. 1 (4): 82. Bibcode:1962ApPhL...1...82H. doi:10.1063/1.1753706

[62] Patel, Neel V. (2014-10-09). "Nobel Shocker: RCA Had the First Blue LED in 1972". IEEE

Spectrum. Institute of Electrical and Electronics Engineers. Retrieved 2015-09-14

[63] Energy Efficiency \& Renewable Energy, “2016 Energy Forcast Summary,” Sep. 2016.

[64] "LED Device Illuminates New Path to Healing" (Press release). nasa.gov. Retrieved January 30, 2012

[65] Tsonev, Dobroslav; Videv, Stefan; Haas, Harald (December 18, 2013). "Light fidelity (Li-Fi):

towards all-optical networking". Proc. SPIE. Broadband Access Communication Technologies VIII. 9007 (2). doi:10.1117/12.2044649

[66] Wiki: Light-emitting diode, 2011

[67] NIOSH, "National Institute for Occupational Safety and Health," 2012.

[68] Jones, H. W. (2012, July 18). Common cause failures and ultra-reliability Presented at the 42nd International Conference on Environmental Systems, San Diego, CA.

[69] Jerath, N. and Ohtakay, H., "Mariner IX Optical Navigation Using Mars Lit Limb," Journal of Spacecraft and Rockets, Vol. 11, No. 7, 1974, pp. 505-511.

[70] Lyons, Daniel T. (August 5-8, 2002). "MARS RECONNAISSANCE ORBITER:

AEROBRAKING REFERENCE TRAJECTORY" (PDF). AIAA/AAS Astrodynamics Specialist

Conference and Exhibit. Retrieved March 9, 2012.

[71] NASA/JPL/Corby Waste - http://photojournal.jpl.nasa.gov/catalog/PIA07241

[72] "MRO HiRISE Camera Specifications". HiRISE website. Retrieved January 2, 2006.

[73] "MRO Context Imager (CTX) Instrument Description". Malin Space Science Systems website. Retrieved June 6, 2006.

[74] "Spacecraft Parts: Instruments: MARCI". MARCI website. Retrieved June 2, 2006.

[75] Selva, D., and Krejci, D., “A survey and assessment of the capabilities of Cubesats for Earth observation," Acta Astronautica, vol. 74, May 2012, pp. 50-68.

[76] "Spacecraft Parts: Instruments: MCS". CRISM Instrument Website. Archived from the original on January 4, 2006. Retrieved May 28, 2006.

[77] Radar Map of Buried Mars Layers Matches Climate Cycles. Keith Cowing, September 22, 2009. 
[78] Byrne, S. et al. 2009. Distribution of Mid-Latitude Ground Ice on Mars from New Impact Craters: $329.1674-1676$

[79] Osterloo, M. et al. 2008. Chloride-Bearing Materials in the Southern Highlands of Mars. Science. 319:1651-1654

[80] Russell, P. et al. (2008). Seasonally active frost-dust avalanches on a north polar scarp of Mars captured by HiRISE. Geophysical Research Letters 35, doi:10.1029/2008GL035790.

[81] NASA/JPL - http://nssdc.gsfc.nasa.gov/nmc/experimentDisplay.do?id=1998-073A-01

[82] NASA - http: http://marsoweb.nas.nasa.gov/HiRISE/instrument.html

[83] G. Swenson, P. Thakker, F. Kamalabadi, M. Frank, V. Coverstone, and H. Voss, Optical sensing of atmospheric emissions with Cubesats and Nanosats, in: Proceedings of SPIE, 2007, vol. 6555, p. 655506.

[84] M. Noca et al., Lessons Learned from the First Swiss Pico-Satellite: SwissCube, in: Proceedings of the 23rd Annual AIAA/USU Con- ference on Small Satellites, 2009.

[85] K.A. Dontchev, K. Ghorakavi, C.E. Haag, T.M. Liu, and R. Ramos, M- Cubed: University of Michigan Multipurpose MiniSatellite with Optical Imager Payload, in: Proceedings of the AIAA Space 2010 Conference \& Exhibition, 2010.

[86] Seitzer, P., Cutler, J., Piergentili, F., Santoni, F., Arena, L., Cardona, T., Cowardin, H., Lee, C., and Sharma, S., "LEDsats: LEO CubeSats with LEDs for Optical Tracking," Advanced Maui Optical and Space Surveillance Technologies Conference, 2016.

[87] Tanaka, T., Kawamura, Y., and Tanaka, T., "Development and operations of nano-satellite FITSAT-1 (NIWAKA),” Acta Astronautica, vol. 107, Feb. 2015, pp. 112-129.

[88] Miranda Straub, "Design and Development of the WVU Advanced Technology Satellite for Optical Navigation," 2015.

[89] Hinkel, H., Zipay, J. J., Strube, M., and Cryan, S., “Technology development of automated rendezvous and docking/capture sensors and docking mechanism for the Asteroid Redirect crewed mission,” Aerospace Conference, 2016 IEEE, IEEE, 2016, pp. 1-8.

[90] Mokuno, M., and Kawano, I., "In-Orbit Demonstration of an Optical Navigation System for Autonomous Rendezvous Docking," Journal of Spacecraft and Rockets, vol. 48, Nov. 2011, pp. 1046-1054.

[91] Dennehy, C. J., and Carpenter, J. R., “A summary of the rendezvous, proximity operations, docking, and undocking (rpodu) lessons learned from the defense advanced research project agency (darpa) orbital express (oe) demonstration system mission,” 2011. 
[92] S. (2012, November 16). Dragon. Retrieved March 17, 2017, from

\section{http://www.spacex.com/dragon}

[93] Underwood, C., Pellegrino, S., Lappas, V. J., Bridges, C. P., and Baker, J., “Using

CubeSat/micro-satellite technology to demonstrate the Autonomous Assembly of a Reconfigurable Space Telescope (AAReST)," Acta Astronautica, vol. 114, Sep. 2015, pp. 112-122.

[94] Kelvinsong: Earth's atmosphere. (2017, March 09). Retrieved March 17, 2017, from https://en.wikipedia.org/wiki/Talk:Earth\%27s_atmosphere

[95] "The height of the tropopause". Das.uwyo.edu. Retrieved 2012-04-18

[96] Journal of the Atmospheric Sciences (1993). "stratopause". Retrieved 2013-10-18.

[97] States, Robert J.; Gardner, Chester S. (January 2000). "Thermal Structure of the Mesopause Region (80-105 km) at $40^{\circ} \mathrm{N}$ Latitude. Part I: Seasonal Variations". Journal of the Atmospheric Sciences 2000. 57: 66-77. Bibcode:2000JAtS...57...66S. doi:10.1175/1520-0469(2000)057

[98] Joe Buchdahl. "Atmosphere, Climate \& Environment Information Programme".

Ace.mmu.ac.uk. Archived from the original on 2010-07-01. Retrieved 2012-04-18.

[99] Randy Russell (2008). "The Thermosphere". Retrieved 2013-10-18

[100] K. Rawer. Wave Propagation in the Ionosphere. Kluwer Acad.Publ., Dordrecht 1993. ISBN 07923-0775-5

[101] Stubbe, 1982

[102] 1Bothmer, V., and Daglis, I. A., Space weather: physics and effects, Berlin ; New York: Springer, 2007.pg 354

[103] Bothmer, V., and Daglis, I. A., Space weather: physics and effects, Berlin ; New York: Springer, 2007.pg 360

[104] Space Age. (2017, March 17). Retrieved March 17, 2017, from https://en.wikipedia.org/wiki/Space_Age

[105] (n.d.). Retrieved March 17, 2017, from https://history.nasa.gov/sputnik/

[106] Martin, G. L., “NewSpace: The Emerging Commercial Space Industry,” 2014.

[107] (n.d.). Retrieved March 20, 2017, from https://www.jwst.nasa.gov/

[108] Garner, R. (2015, January 15). Hubble Space Telescope. Retrieved March 20, 2017, from https://www.nasa.gov/mission_pages/hubble/main/index.html

[109] Selva, D., and Krejci, D., “A survey and assessment of the capabilities of Cubesats for Earth observation," Acta Astronautica, vol. 74, May 2012, pp. 50-68. 
[110] OPAL - eoPortal Directory - Satellite Missions. (n.d.). Retrieved March 20, 2017, from https://directory.eoportal.org/web/eoportal/satellite-missions/o/opal

[111] Manchester, Z., Peck, M., and Filo, A., "Kicksat: a crowd-funded mission to demonstrate the world's smallest spacecraft," 2013.

[112] Space Systems Design Studio. (n.d.). Retrieved March 20, 2017, from

http://www.spacecraftresearch.com/MII/MII_overview.html

[113] National Aeronautics and Space Administration, "Dellingr 6U CubeSat."

[114] Doncaster, B., Shulman, J., Bradford, J., and Olds, J., "SpaceWorks 2016 Nano-Microsatellite Market Forcast." $30^{\text {th }}$ Annual AIAA/USU Conference on Small Satellites

[115] Clark, C., "Clyde Space CEO No Slowdown in CubeSat,” Aug. 2016.

[116] www.sciencemag.org, "Science-2014-Feature-1449."

[117] CubeSat. (2017, March 19). Retrieved March 20, 2017, from

https://en.wikipedia.org/wiki/CubeSat\#Launch_and_deployment

[118] Crook, M. R., “NPS CubeSat launcher design, process and requirements,” Monterey, California. Naval Postgraduate School, 2009.

[119] Clark, C., and Logan, R., "Power budgets for mission success," CubeSat Workshop, 2011.

[120] CubeSat Constellation around Mars. (n.d.). Retrieved March 20, 2017, from

http://ccar.colorado.edu/asen5050/projects/projects_2013/Naik_Siddhesh/Cubesats.html

[121] Home. (n.d.). Retrieved March 20, 2017, from http://nanoracks.com/

[122] NanoRacks, L. L. C., "NanoRacks CubeSat Deployer (NRCSD) Interface Control

Document," 0.36, 2013.

[123] SatMagazine. (n.d.). Retrieved March 20, 2017, from

http://www.satmagazine.com/story.php?number=602922274

[124] Jackson, S. (2017, February 17). About CubeSat Launch Initiative. Retrieved March 20, 2017, from https://www.nasa.gov/content/about-cubesat-launch-initiative

[125] A Nation of Makers. (n.d.). Retrieved March 20, 2017, from

https://www.whitehouse.gov/nation-of-makers

[126] Morris, J., Zemerick, S., Grubb, M., Lucas, J., Jaridi, M., Gross, J. N., Christian, J. A., Vassiliadis, D., Kadiyala, A., Dawson, J., others, and Pachol, M., "Simulation-to-Flight 1 (STF-1): A Mission to Enable CubeSat Software-based Verification and Validation," 54th AIAA Aerospace Sciences Meeting, 2016, p. 1464. 
[127] Robb, A. (n.d.). Ionosphere: Definition, Facts, Temperature \& Layers. Retrieved June 21, 2017, from http://study.com/academy/lesson/ionosphere-definition-facts-temperature-layers.html [128] Manasreh O 2000 III-Nitride Semiconductors: Electrical, Structural and Defects Properties (Amsterdam: Elsevier) p 37

[129] Kordos P, Morvic M, Betko J, Van Hove J M, Wowchak A M and Chow P P 2000

Conductivity and Hall effect characterization of highly resistive molecular-beam epitaxial GaN layers J. Appl. Phys. 88 5821-6

[130] Erol M 2000 Temperature dependence of transport characteristics of wurtzite GaN epilayers Czech. J. Phys. 50 665-70

[131] Zhao, Y., Zhong, W., Liu, J., Huang, Z., and Wei, A., "Junction temperature measurement of GaN-based light-emitting diodes using temperature-dependent resistance," Semiconductor Science and Technology, vol. 29, Mar. 2014, p. 035008.

[132] Chhajed S, Xi Y, Gessmann T, Xi J Q, Shah J M, Kim J K and Schubert E F 2005 Junction temperature in light-emitting diodes assessed by different methods Proc. SPIE 5739, Light-Emitting Diodes: Research, Manufacturing and Applications IX (San Jose, CA, 25-27 Jan.) pp 16-24

[133] He, S. M., Luo, X. D., Zhang, B., Fu, L., Cheng, L. W., Wang, J. B., and Lu, W., "Junction temperature measurement of light emitting diode by electroluminescence," Review of Scientific Instruments, vol. 82, 2011, p. 123101.

[134] S. J. Cai, Y. S. Tang, R. Li, Y. Y. Wei, L. Wong, Y. L. Chen, K. L. Wang, M. Chen, Y. F. Zhao, R. D. Schrimpf, J. C. Keay, and K. F. Galloway, IEEE Trans. Electron Dev., 47, 304 (2000). [135] A. Y. Polyakov and In-Hwan Lee, Mat. Sci. Eng. R, 94, 1 (2015).

[136] A. Y. Polyakov, N. B. Smirnov, A. V. Govorkov, N. V. Pashkova, S. J. Pearton, J. M. Zavada, and R. G. Wilson, J. Vac. Sci. Technol. B 21, 2500 (2003)

[137] C.-F. Lo, L. Liu, F. Ren, S. J. Pearton, B. P. Gila, H.-Y. Kim, J. Kim, O. Laboutin, Y. Cao, J. W. Johnson, and I. I. Kravchenko, J. Vac. Sci. Technol. B 30, 041206 (2012).

[138] B. Luo, J. Kim, F. Ren, J. K. Gillespie, R. C. Fitch, J. Sewell, R. Dettmer, G. D. Via, A. Crespo, T. J. Jenkins, B. P. Gila, A. H. Onstine, K. K. Allums, C. R. Abernathy, S. J. Pearton, R. Dwidevi, T. N. Fogarty, and R.Wilkins, Appl. Phys. Lett. 82, 1428(2003). 


\section{Ch. 2 References}

[127] S.r.o., J. (n.d.). Retrieved November 25, 2017, from

http://www.pion.cz/en/article/electromagnetic-spectrum

[128] P.-C. Wang, C.-L. Lin, Y.-K. Su, P.-C. Chien, G.-S. Huang, S.-C. Kuo and G.-C. Lyu, "Effects of TiO2-doped silicone encapsulation material on the light extraction efficiency of GaN-based blue light-emitting diodes," Thin Solid Films, 2014.

[129] https://micro.magnet.fsu.edu/primer/lightandcolor/images/ledintrofigure6.jpg.

[130] S. Yi, W. J. Chung and J. Heo, "Stable and Color-Tailorable White Light from Blue LEDs Using Color-Converting Phosphor Glass Composites," Journal of American Ceramic Society, vol. 97, no. 2, pp. 342-345, 2014.

[131] Di Carlo, A. (2001). "Tuning Optical Properties of GaN-Based Nanostructures by Charge Screening". Physica status solidi (a). 183: 81-85. doi:10.1002/1521-396X(200101)183:1 Arakawa, Y. (2002). "Progress in GaN-based quantum dots for optoelectronics applications". IEEE Journal of Selected Topics in Quantum Electronics. 8 (4): 823-832. doi:10.1109/JSTQE.2002.801675.

[132] Solid State, https://en.wikipedia.org/wiki/File:Wurtzite_polyhedra.png

[133] Benjah-bmm27, https://commons.wikimedia.org/wiki/File:Sphalerite-unit-cell-depth-fade3D-balls.png

[134] TheNoise https://en.wikipedia.org/wiki/P\%E2\%80\%93n_junction\#/media/File:Pn-junctionequilibrium.png

[135] Paul Greenland, Technologist and Tushar Dhayagude, Co-Founder \& Vice President mSilica Inc., Santa Clara, California, USA. (n.d.). LED Action. Retrieved November 25, 2017, from https://www.powersystemsdesign.com/led-action/36 [136] B. H. Rose and C. E. Barnes, J. Appl. Phys. 53, 1772 (1982)

[137] A. H. Johnson, IEEE Trans. Nucl. Sci. 60, 2054 (2013)

[138] E.S.Yang,C.M.Wu,R.Y.Hung,J.Appl.Phys.51(1980)1262., S.M.Sze,

PhysicsofSemiconductorDevices, 2nded., Wiley, NewYork, 1981.

[139] Xi, Y., and Schubert, E. F., "Junction-temperature measurement in GaN ultraviolet lightemitting diodes using diode forward voltage method," Applied Physics Letters, vol. 85, 2004, pp. 2163-2165.

[140] Wang, J.-C., Fang, C.-H., Wu, Y.-F., Chen, W.-J., Kuo, D.-C., Fan, P.-L., Jiang, J.-A., and Nee, T.-E., "The effect of junction temperature on the optoelectrical properties of InGaN/GaN multiple quantum well light-emitting diodes," Journal of Luminescence, vol. 132, Feb. 2012, pp. 429-433. 
[141] J. R. Srour, S. C. Chen, S. Othmer, and R. A. Hartmann, "Radiation damage coefficients for silicon depletion regions," Trans. Nucl. Sci., vol. 26, p. 4784, 1979.

[142] J. R. Srour, R. A. Hartmann, and K. S. Kitazaki,” Permanent damage produced by single proton interactions in silicon devices," Trans. Nucl. Sci., vol. 33, p. 1597, 1986.

[143] http://beam.helsinki.fi/ knordlun/mdh/rangetext.html, Kai Norlund

[144] M. Sze, Physics of Semiconductors (Wiley-Interscience, New York, 1969), p. 104

\section{Ch. 3 References}

[144] Proton damage effects on light emitting diodes B. H. Rose and C. E. Barnes Sandia National Laboratories. a) Albuquerque. New Mexico 87185

[145] Ian Poole, http://www.radio-electronics.com/info/data/semicond/schottky diode/schottkydiode-iv-characteristic.gif

[146] Dglr6328 https://commons.wikimedia.org/wiki/File:Electroluminescent panel spectrum.png [147] "What is Conformal Coating?". www.electrolube.com. Retrieved 11 June 2015.

[148] D. (n.d.). Retrieved November 25, 2017, from

http://blog.paryleneconformalcoating.com/download-parylene-101--coating-facts-sheet

[149] Pumpkin Inc., 09/26/03, CubeSat Kit PCB Specification

[150] KYOCERA, Retrieved 11/25/2017, C-QFJ (CERAMIkC QUAD FLAT J-LEADED

\section{PACKAGES)}

[151] 3MTM, 9/14/2011, Chip Carrier Sockets Low Profile, Four-Row, Through Hole

[152] Harwin, 9/20/05, M20-7830346-datasheet.pdf (Header pins - Female).

[153] Harwin, 9/20/05, M20-7830346-datasheet.pdf (Header pins - Male).

[154] SAMTEC, Retrieved 11/25/2017, esq-126-39-g-d (CubeSat Stack Connection)

[155] Pachol, Matthew, Anand Kadiyala, Dimitris Korakakis, and Jeremy M. Dawson. "LOCC -

Enabling the Characterization of Minamally Shielded LEDs.” 30th Annual AIAA/USU Conference on Small Satellites, August 1, 2016.

[156] Arduino - ArduinoBoardMega2560. (n.d.). Retrieved March 30, 2017, from https://www.arduino.cc/en/Main/arduinoBoardMega2560

[157] Microchip, Retrieved 11/25/2017, Mode, E. M., "MCP4901, with SPI Interface”.

[158] Analog Devices, Retrieved 11/25/2017, Rail-to-Rail, High Output Current Amplifier, AD8397

[159] Analog Devices, Retrieved 11/25/2017, $1.5 \Omega$ On Resistance, $\pm 15 \mathrm{~V} /+12 \mathrm{~V} / \pm 5 \mathrm{~V}$, iCMOS, Quad SPST Switches, ADG1412 
[160] Microchip, Retrieved 11/25/2017, 16-Bit I/O Expander with Open-Drain Outputs, MCP23018

[161] Texas Instruments, Retrieved 11/25/2017, Zerø-Drift, Bi-Directional CURRENT/POWER MONITOR with I2C ${ }^{\text {TM }}$ Interface, INA219

[162] MAZe'T, Retrieved 11/25/2017, 16 bit 4-channel analog-to-digital converter (ADC) with I2C control/output, MCDC04

[163] MAZeT, Retrieved 11/25/2017, Integral True Color Sensor, MTCSiCF True Color Sensor [164] CIE (1932). Commission internationale de l'Eclairage proceedings, 1931. Cambridge:

Cambridge University Press.

[165] CIE 1931 color space. (2017, November 13). Retrieved November 25, 2017, from https://en.wikipedia.org/wiki/CIE_1931_color_space\#/media/File:CIE1931xy_blank.svg [166] BenRG - File:CIExy1931.svg

[167] CUI INC., Retrieved 11/25/2017, DC-DC CONVERTER, PDS2-M

[168] Micrel, Retrieved 11/25/2017, Ultra-low quiescent current 150mA uCap Low Dropout Regulator, MIC5225

[168] Texas Instruments, Retrieved 11/25/2017, ISO154x Low-Power Bidirectional I2C Isolators, ISO1540

[169] PA, G., "ATmega2560, 1. Pin Configurations."

[170] MAZeT, Retrieved 11/25/2017, JENCOLOR® - Sensor Board with I2C-Interface, MTCSINT-AB4

[171] NXP, 19 August 2013, 2-bit bidirectional low voltage translator, GTL2002

[172] Julia, lia (20 November 2011). "PCB assembly, PCB assembly quote, Printed Circuit Board Assembly". www.ourpcb.com. OURPCB. Retrieved 29 December 2016.

[173] The Internet of Everyday Things CS194-05, Spring 2008. (n.d.). Retrieved November 25, 2017, from http://www.cs.berkeley.edu/ prabal/teaching/cs194-05-s08/ Printed Circuit Board Design Flow Methodology

[174] Tavernier, Karel. "PCB Fabrication Data - A Guide". Ucamco. Retrieved 8 January 2015.

[175] PixelTeq, Retrieved 11/25/2017, PixelSensor Multispectral Sensors

[176] (n.d.). Retrieved November 25, 2017, from https://opensource.gsfc.nasa.gov/projects/cfe/ [177] NASA, Retrieved 11/25/2017, NASA OPEN SOURCE AGREEMENT VERSION 1.3 


\section{Ch. 4 References}

[178] D.-H. Kim, C.-O. Cho, Y. S. Park, J. Cho, J. S. Im, Y. Park, W. J. Choi and Q.-H. Park, "Enhanced light extraction from GaN-based light-emitting diodes with holographically generated two-dimensional photonic crystal patterns," Applied Physics Letters, vol. 87, p. 203508, 2005.

[179] J. J. Wierer, A. David and M. M. Megens, "III-nitride photonic-crystal light-emitting didoes with high extraction efficiency," Nature Photonics, vol. 3, pp. 163-169, 2009.

[180] http://www.powerwaywafer.com/Epitaxial-Wafer.html

[181] L. Rodak and D. Korakakis, "Aluminum Gallium Nitride Alloys grown via Metalorganic Vapor Phase Epitaxy using a digital growth technique," Journal of Electronic Materials, vol. 40, no. 4, pp. 388-393, April, 2011.

[182] L. E. Rodak, C. M. Miller and D. Korakakis, "Surfactant effects of Indium on cracking in AlN/GaN distributed bragg reflectors grown via metal organic vapour phase epitaxy," Superlattices and Microstructures, vol. 49, no. 1, pp. 52-59, January 2011.

[183] DISCO, Retrieved 11/25/2017, Electroformed Bond Hub Blades, ZH05 SERIES

[184] EPOXY TECHNOLOGY, Retrieved 11/25/2017, EPO-TEK 353ND

[185] EPOXY TECHNOLOGY, Retrieved 11/25/2017, EPO-TEK 301-2FL 


\section{Appendix}

I-V Characteristics of LOCC output for each LED.

\section{A-1. Chip Set 1}

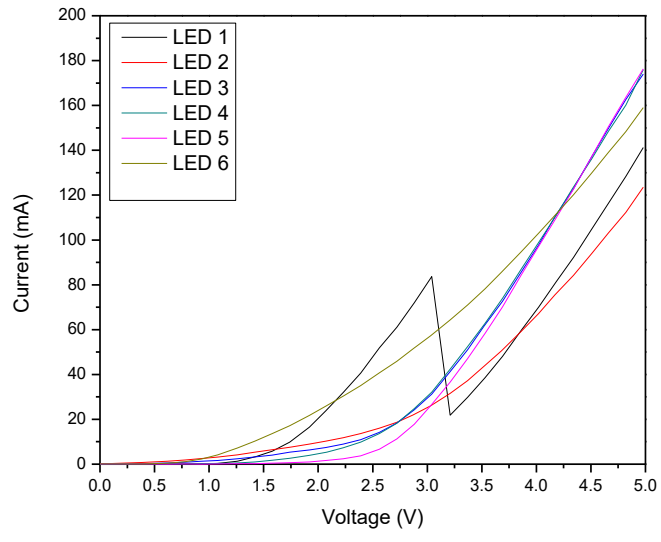

Trial 1

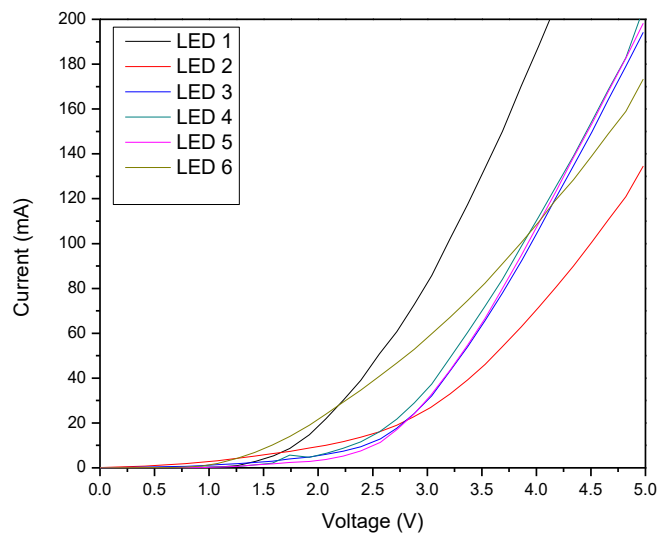

Trial 3

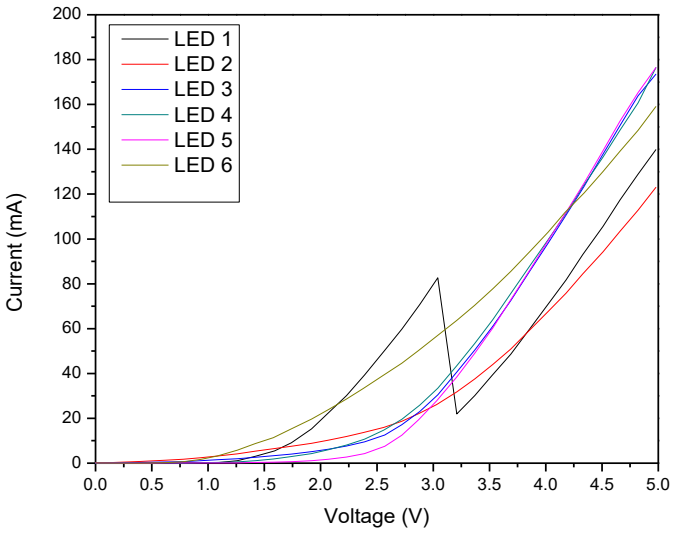

Trial 2

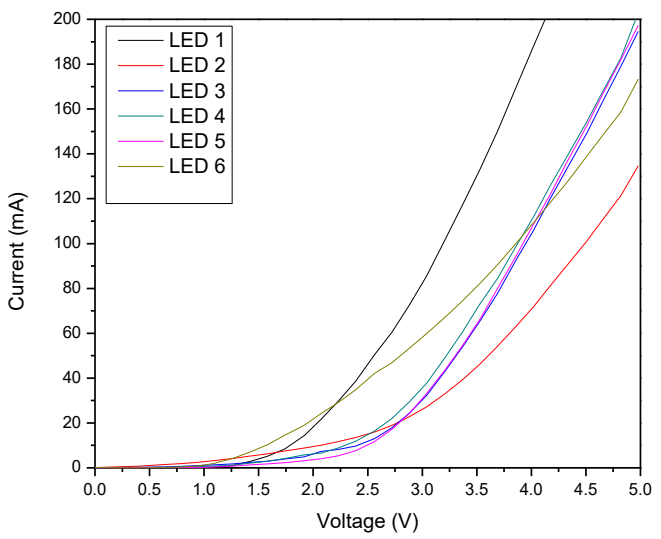

Trial 4

Trials 1-4, I-V Characteristics of Chip Carrier 1 


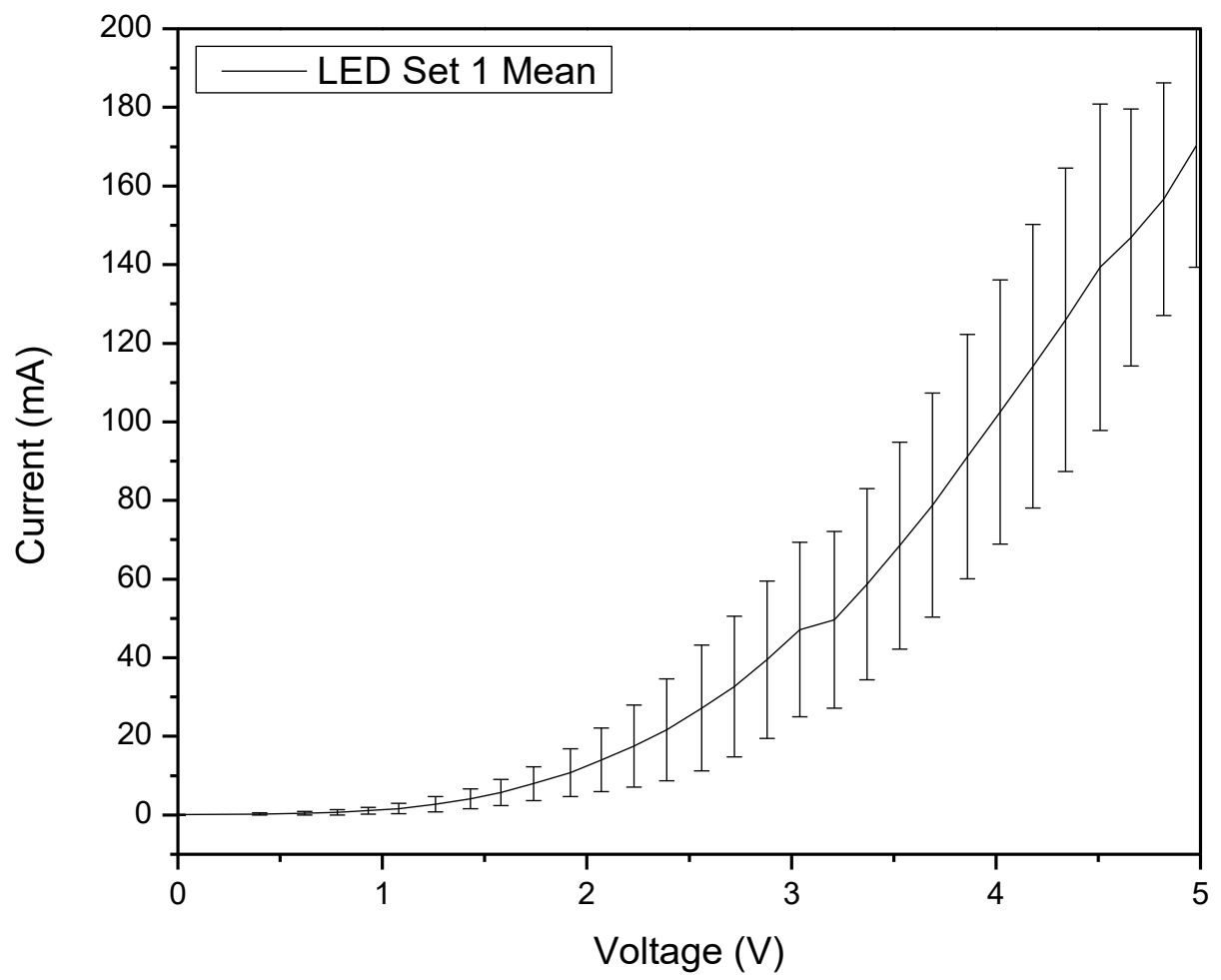

Chip Carrier Set 1 Average and Mean Standard Deviation

A-2. Set 3 


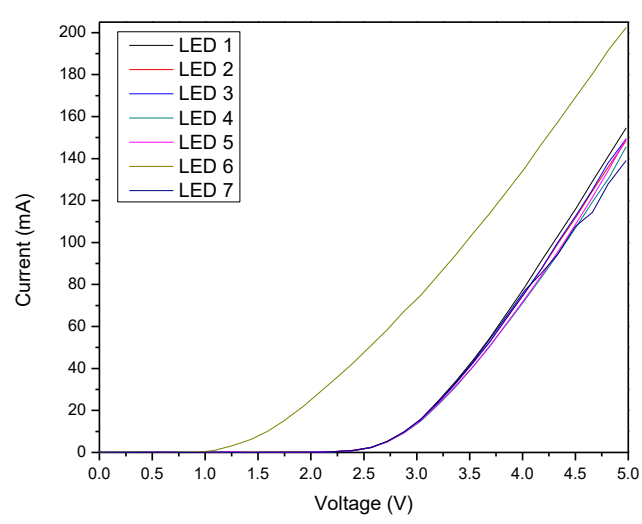

Trial 1

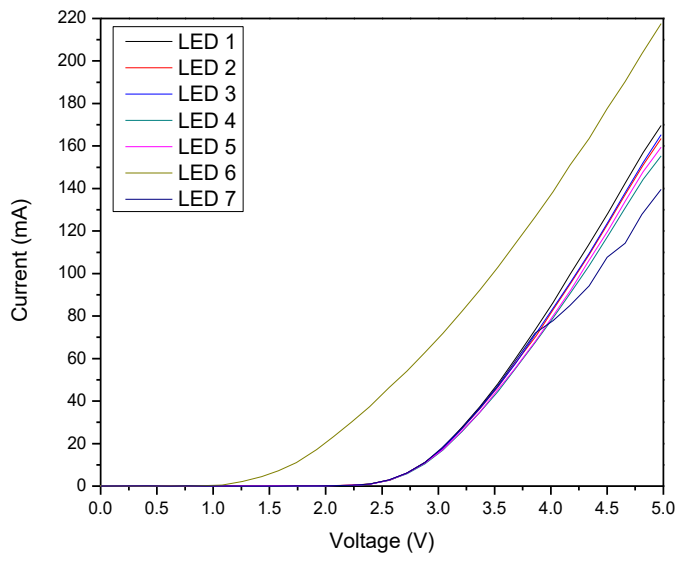

Trial 3

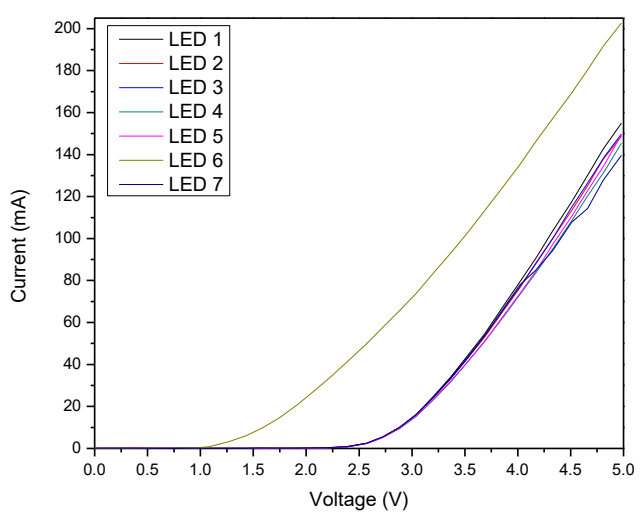

Trial 2

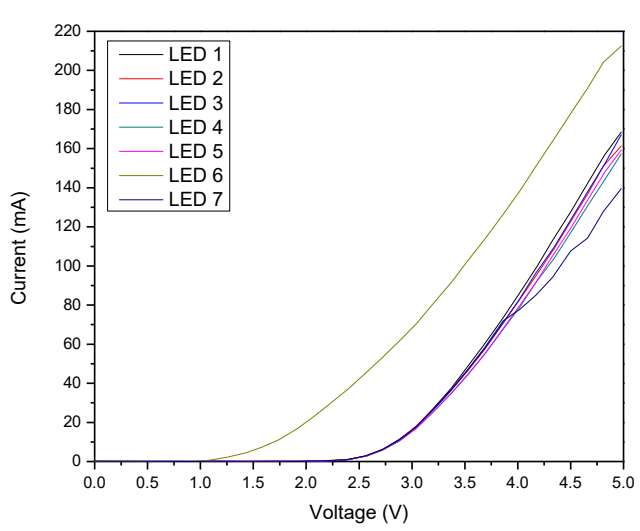

Trial 4

Trials 1-4, I-V Characteristics of Chip Carrier 3 


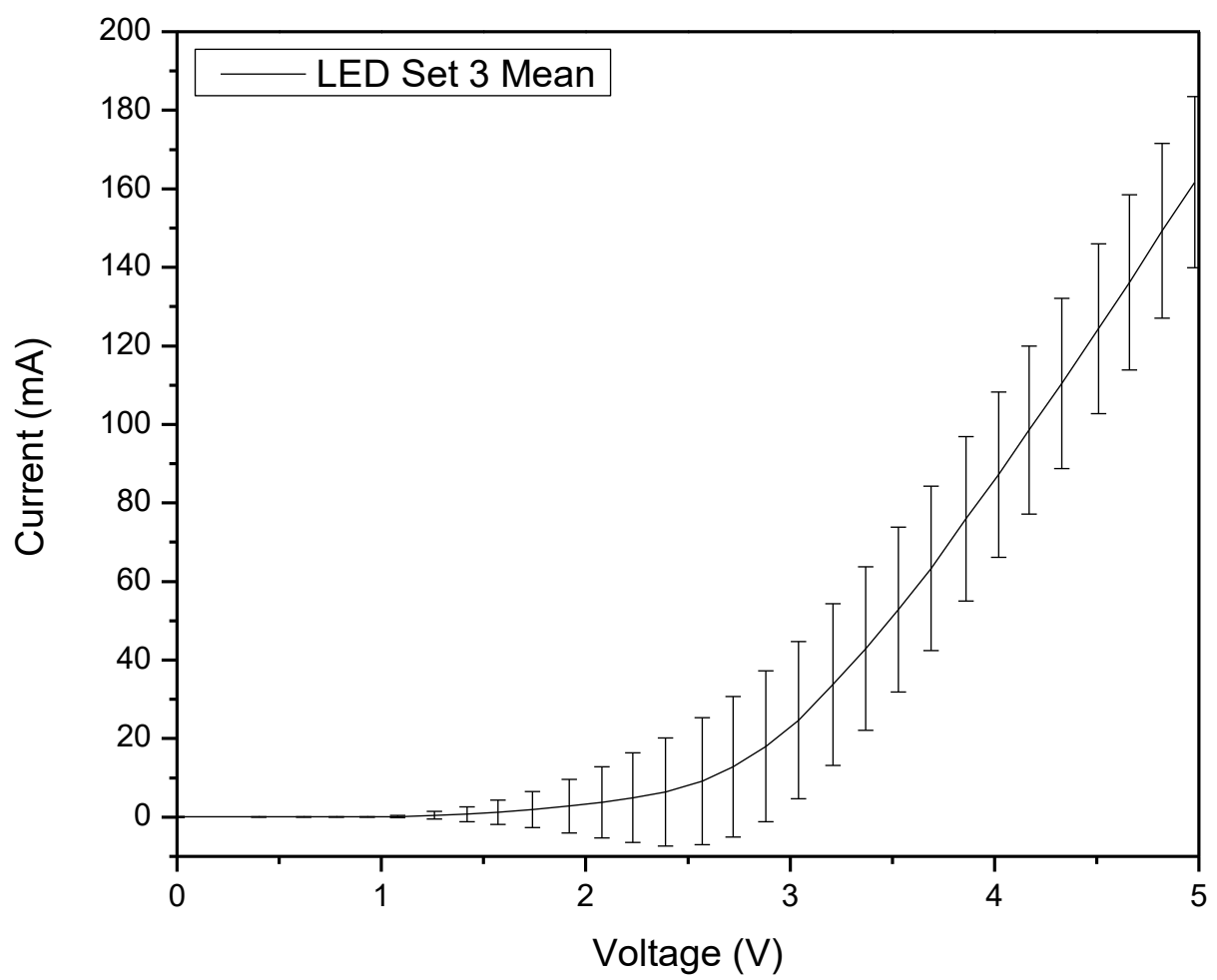

Chip Carrier Set 3 Average and Mean Standard Deviation 
I-V characteristics of individual LEDs. Results 1-7 are listed in body of thesis. These results indicate the results over four trials alongside the mean and standard deviations of each individual LED.

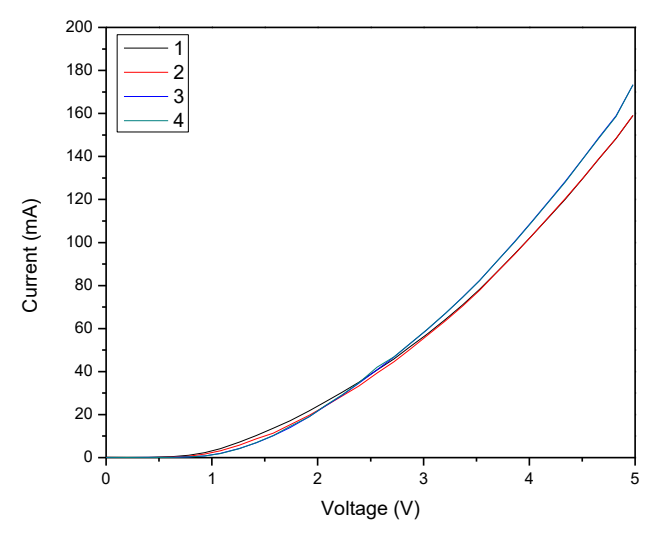

LED 8 - 4 Trials

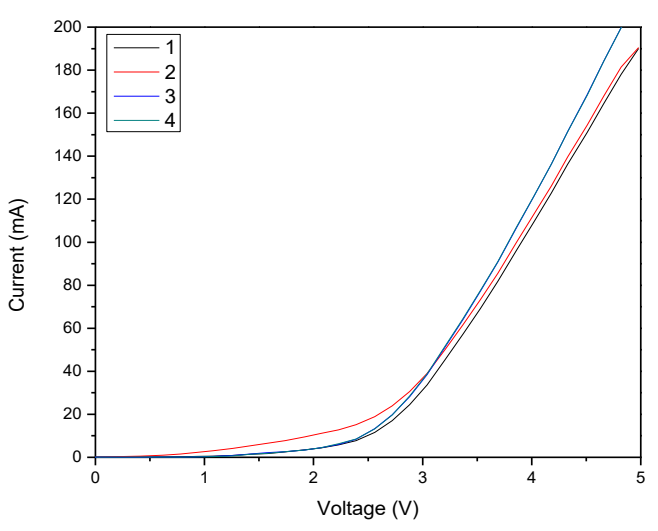

LED $9-4$ Trials

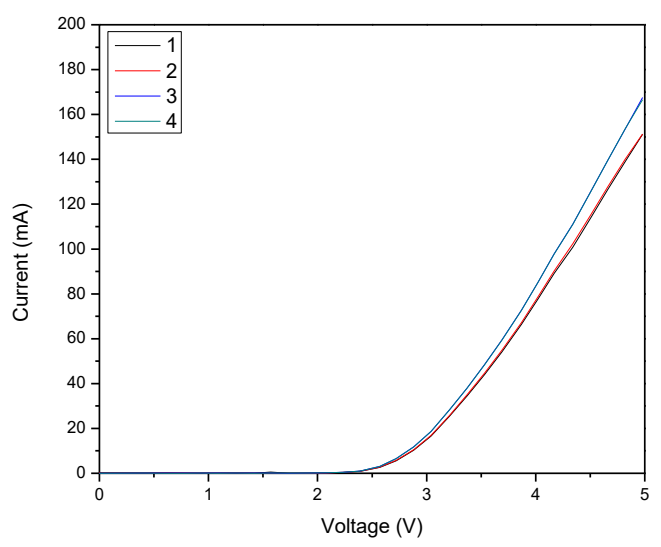

LED 10 - 4 Trials

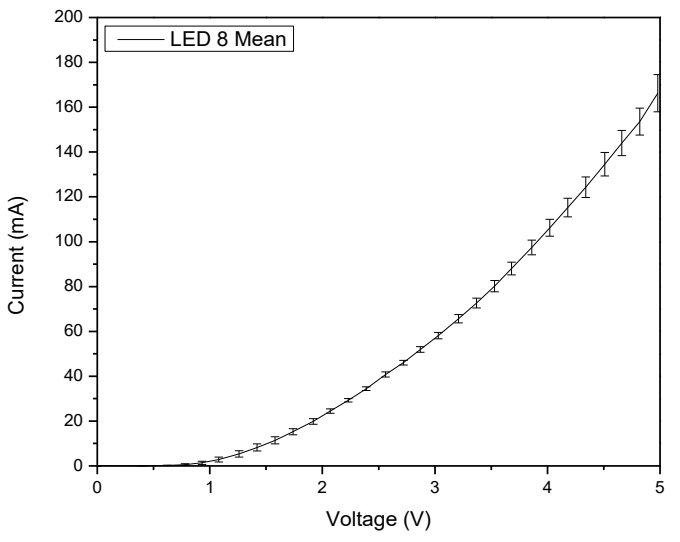

LED 8 - Mean and Standard Deviation

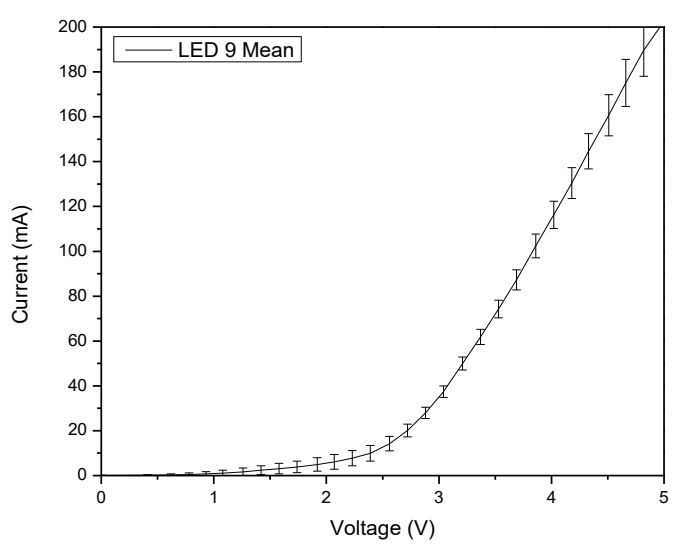

LED 9 - Mean and Standard Deviation

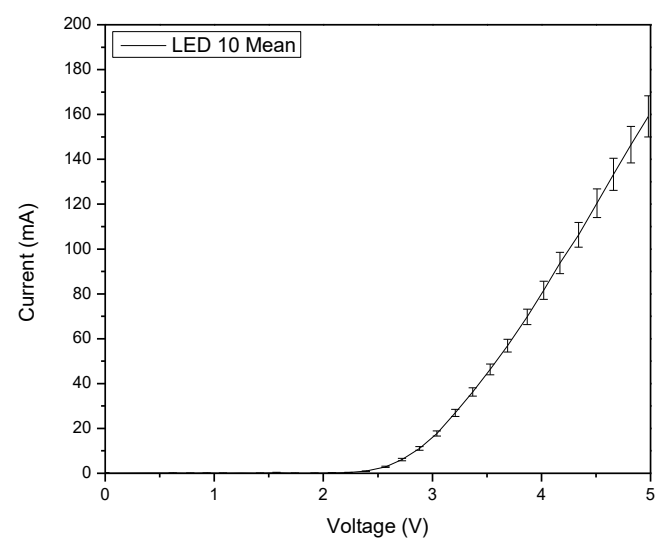

LED 10 - Mean and Standard Deviation

LED 8 - 10: 4 trials and Mean and Standard Deviation 


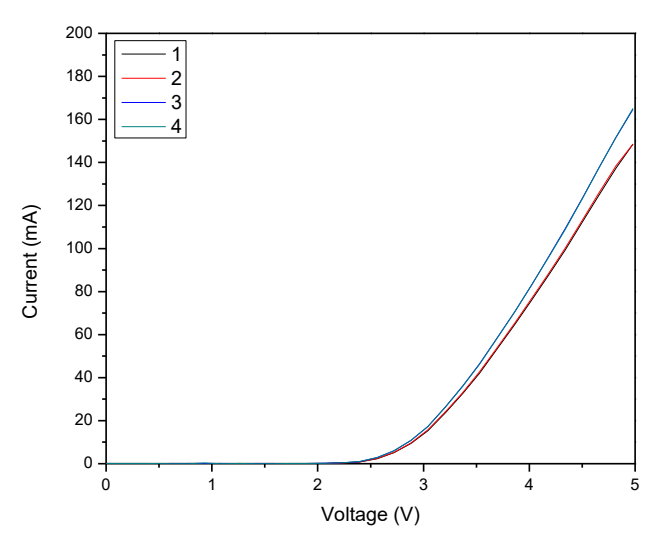

LED $12-4$ Trials

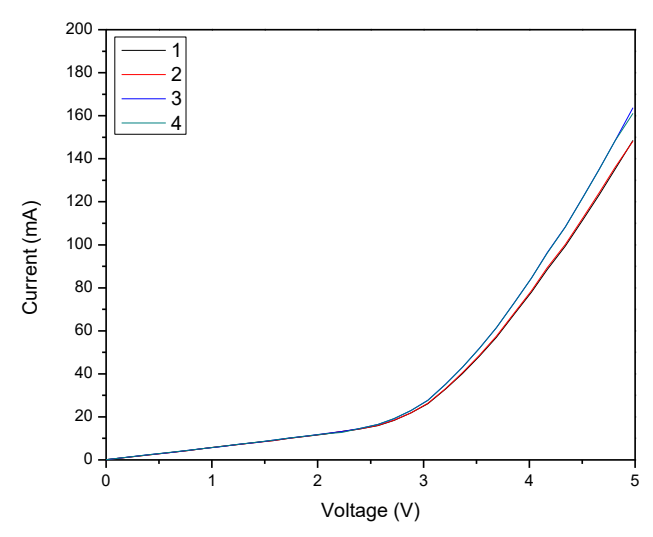

LED $14-4$ Trials

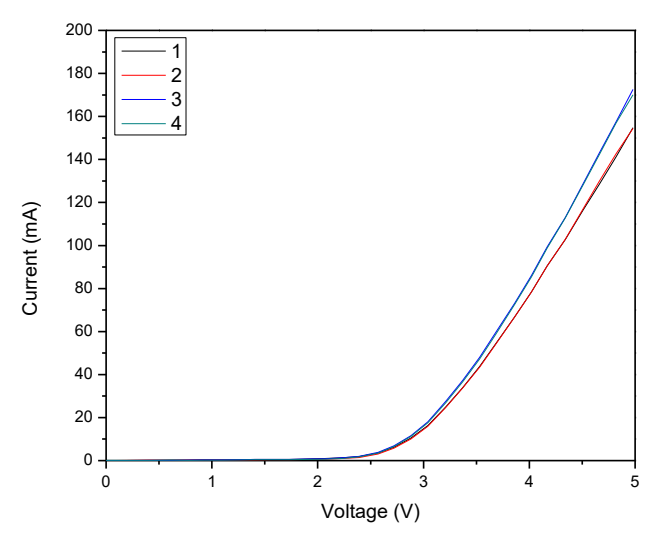

LED $15-4$ Trials

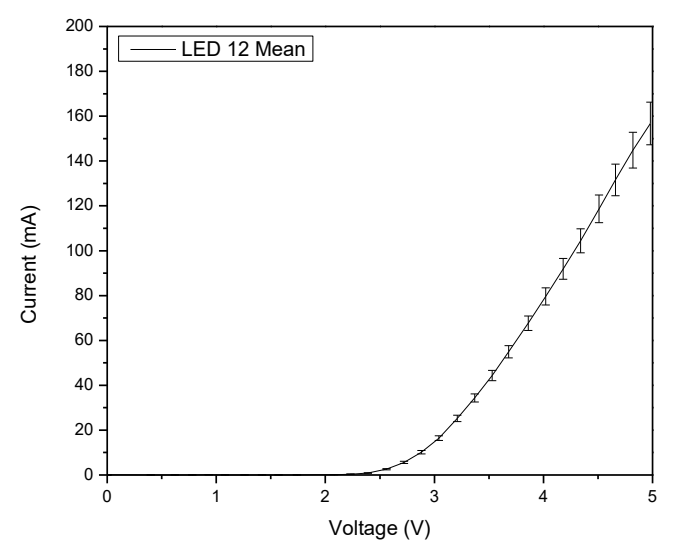

LED 12 - Mean and Standard Deviation

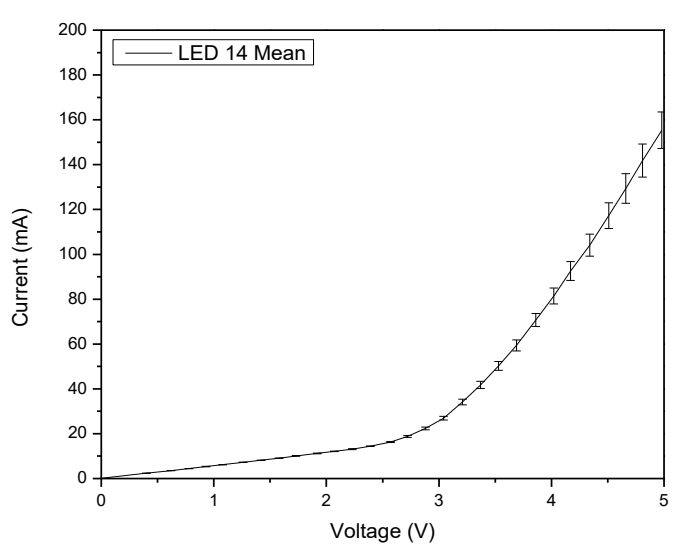

LED 14 - Mean and Standard Deviation

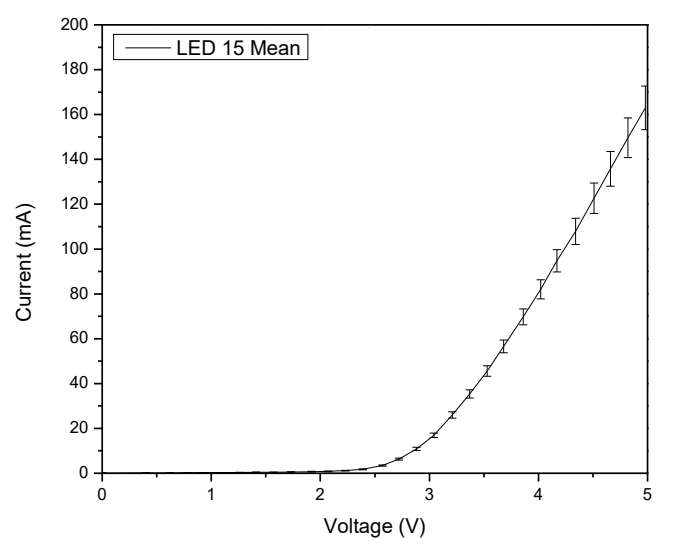

LED 15 - Mean and Standard Deviation

LED 12 - 15: 4 trials and Mean and Standard Deviation 


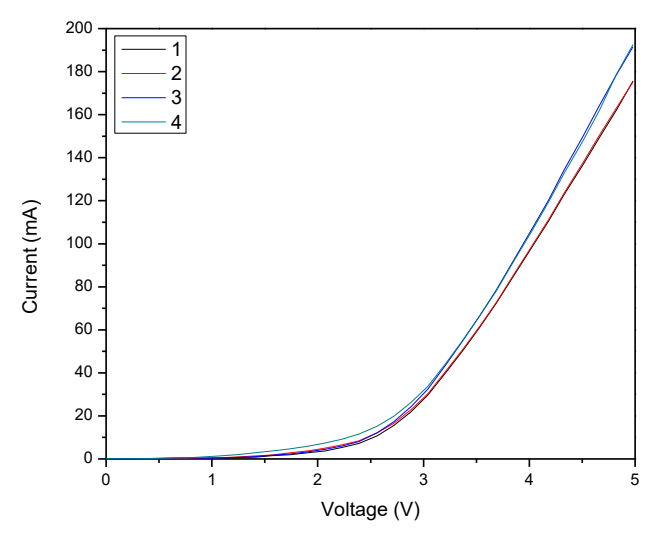

LED 16 - 4 Trials

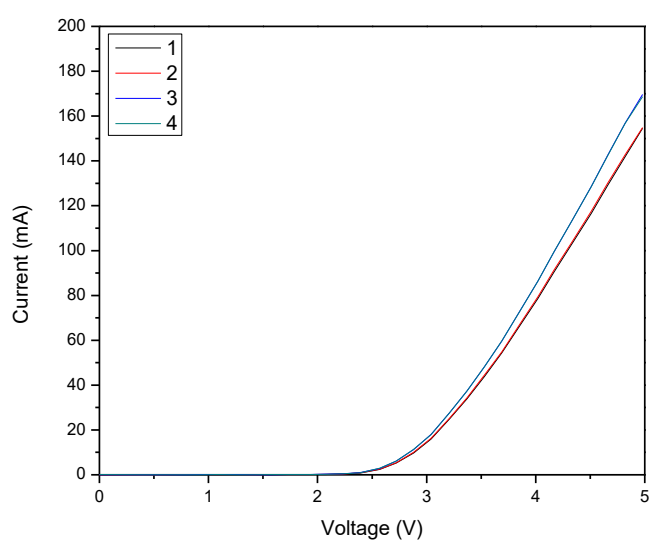

LED 17 - 4 Trials

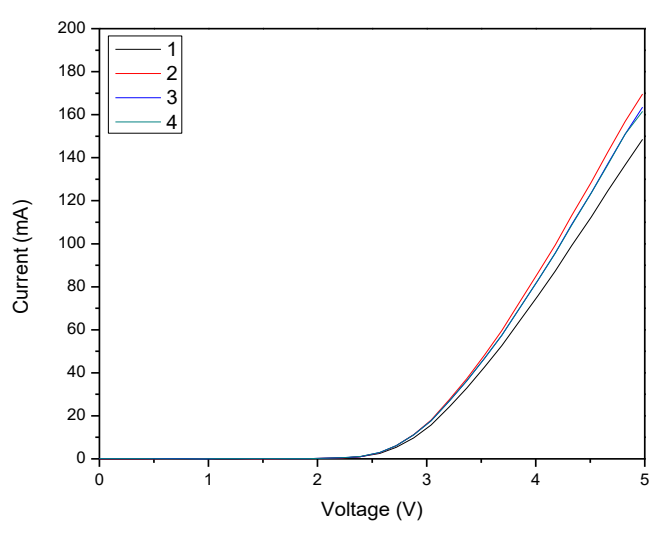

LED 18 - 4 Trials

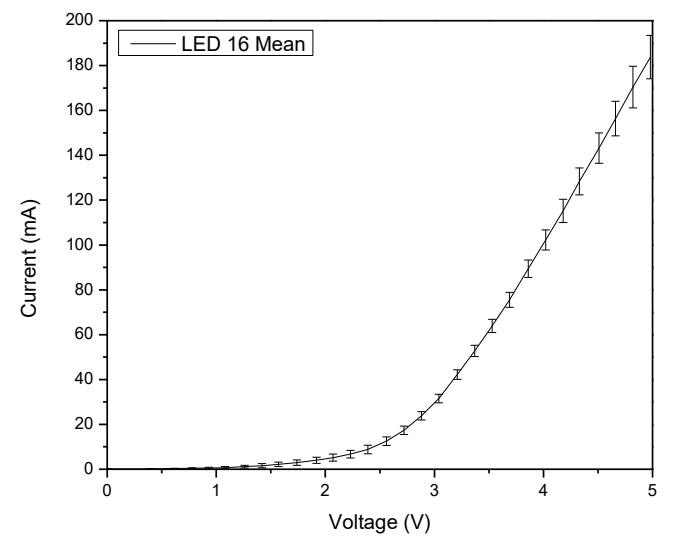

LED 16 - Mean and Standard Deviation

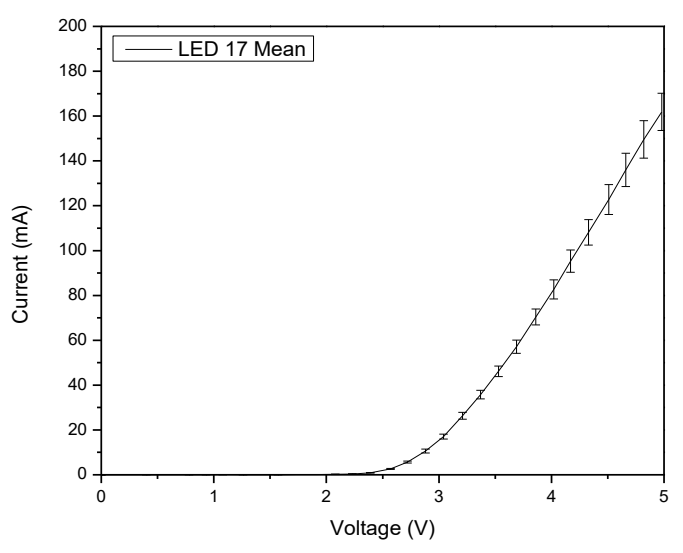

LED 17 - Mean and Standard Deviation

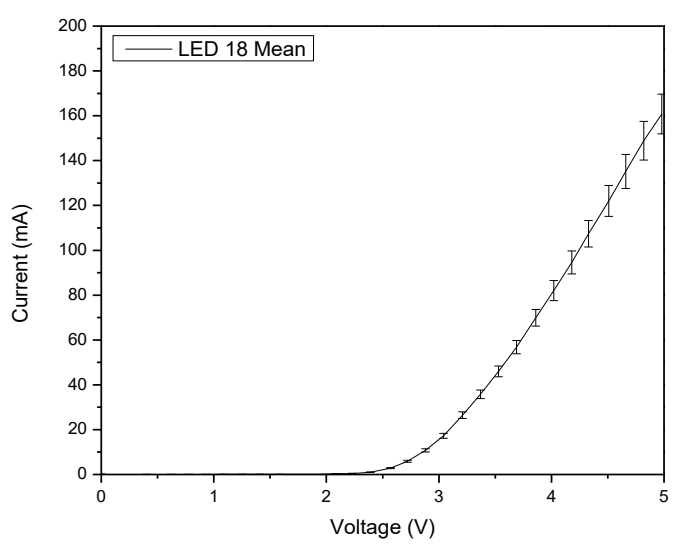

LED 18 - Mean and Standard Deviation

LED 16 - 18: 4 trials and Mean and Standard Deviation 


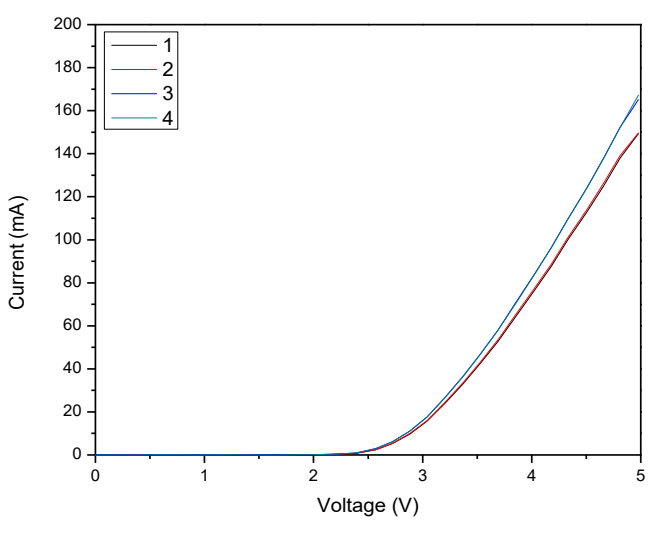

LED $19-4$ Trials

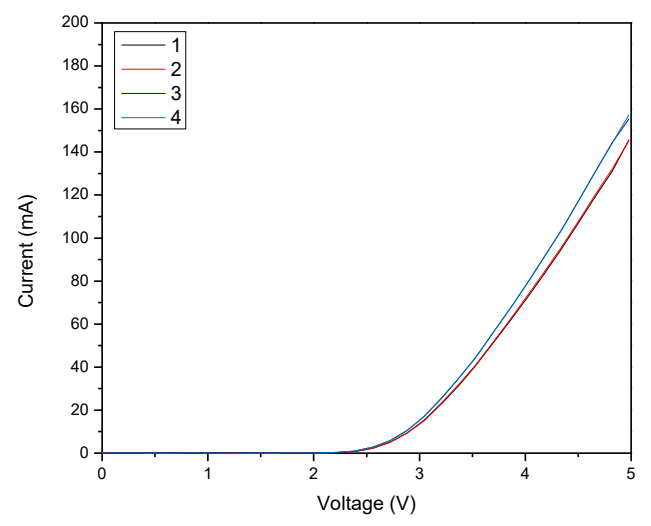

LED $20-4$ Trials

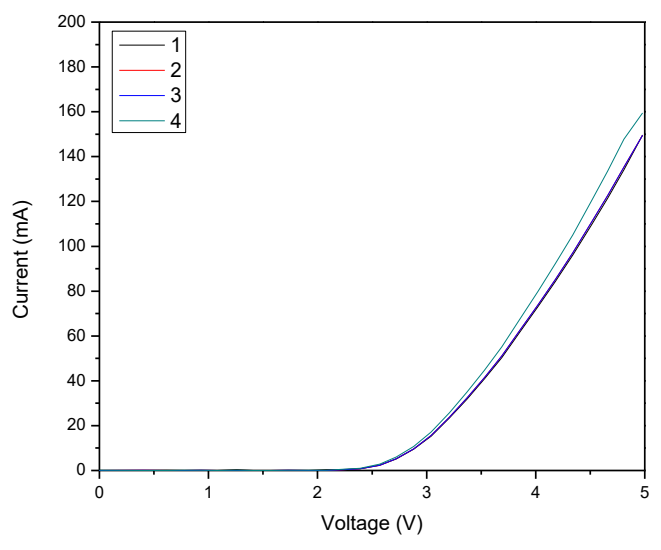

LED $21-4$ Trials

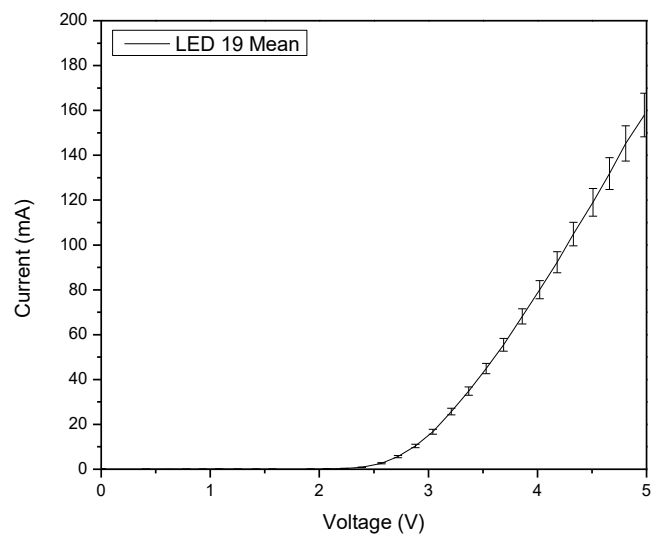

LED 19 - Mean and Standard Deviation

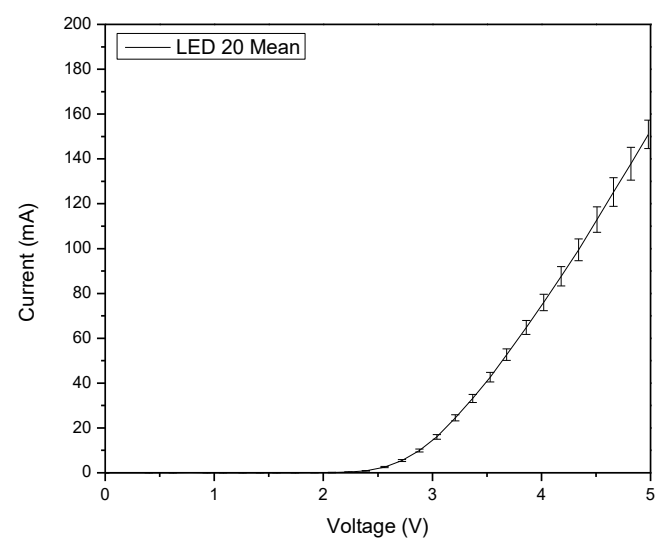

LED 20 - Mean and Standard Deviation

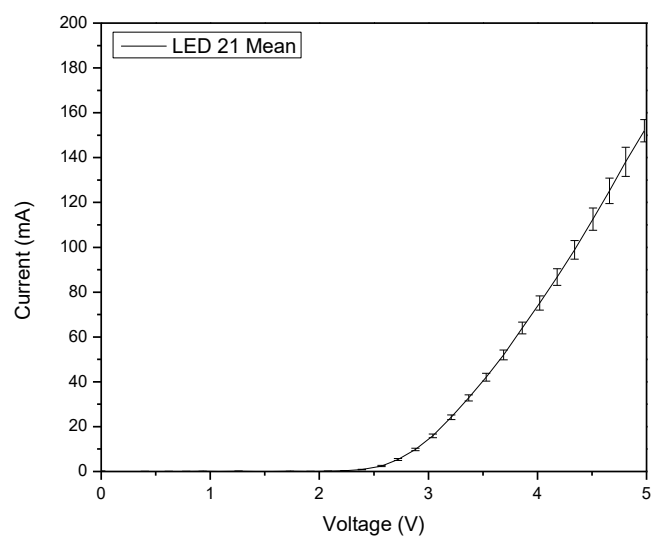

LED 21 - Mean and Standard Deviation

LED 19 - 21: 4 trials and Mean and Standard Deviation 


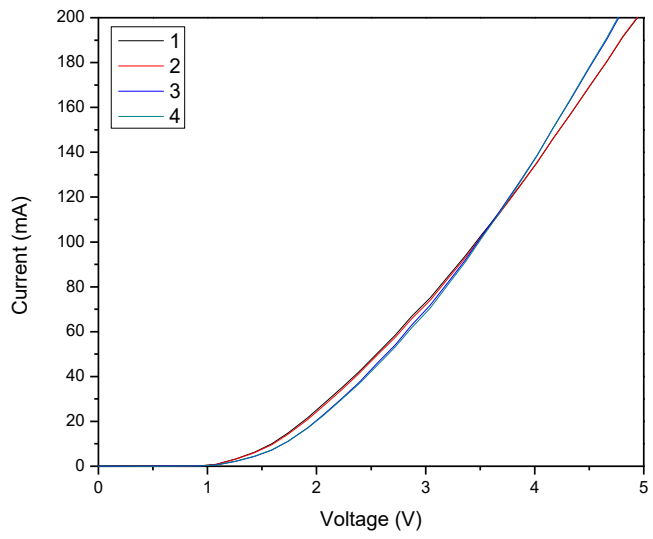

LED $23-4$ Trials

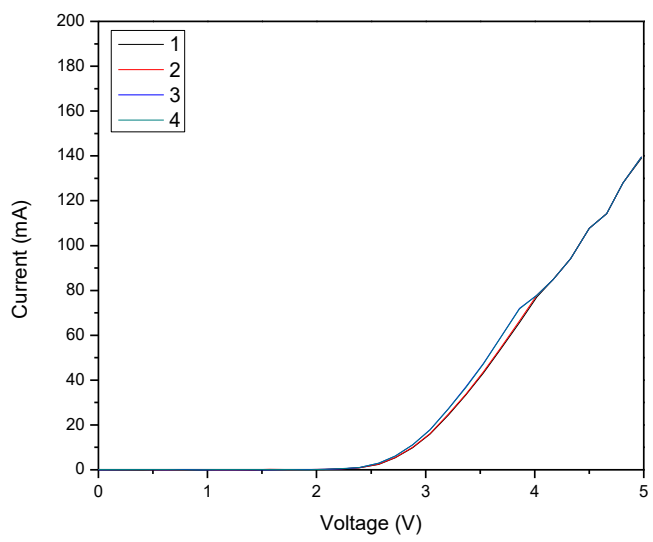

LED $24-4$ Trials

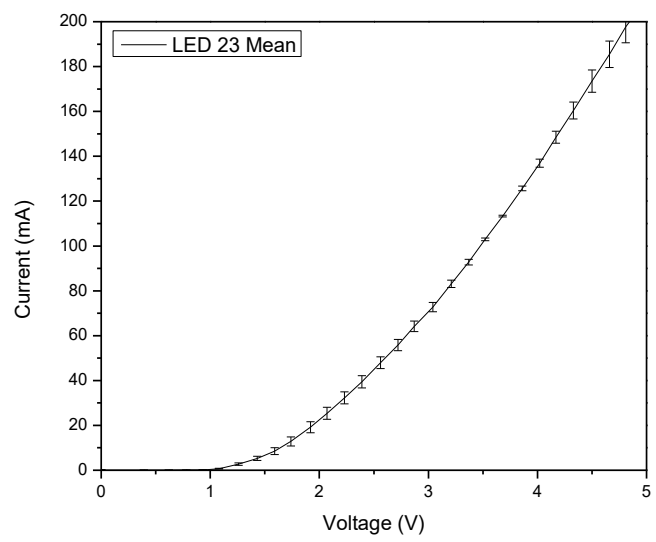

LED 23 - Mean and Standard Deviation

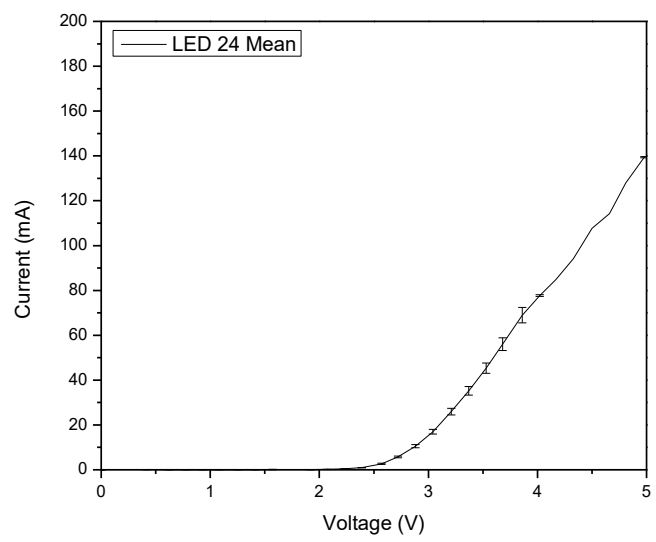

LED 24 - Mean and Standard Deviation

LED 23 - 24: 4 trials and Mean and Standard Deviation 


\section{A-3. Normalized Light Output of Individual LEDs}

Figures depicting the normalized light output of all LEDs.

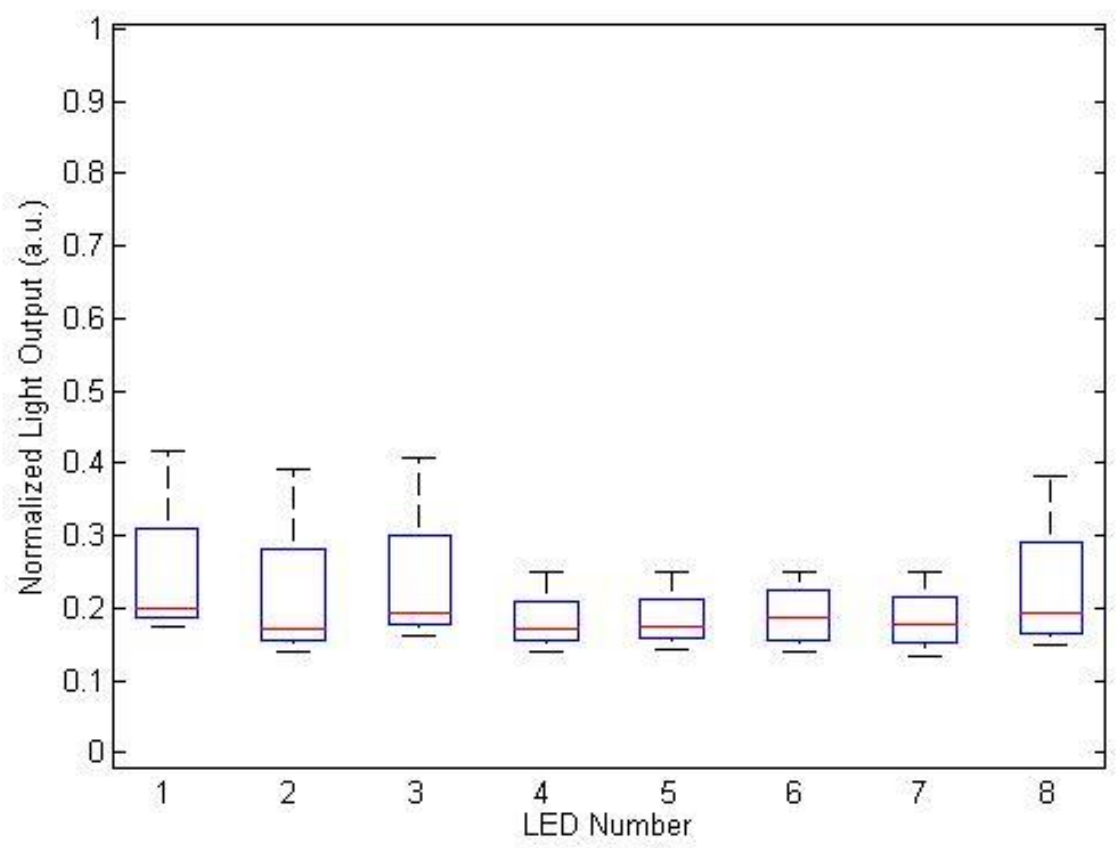

LED Chip 1 Normalized Light Output

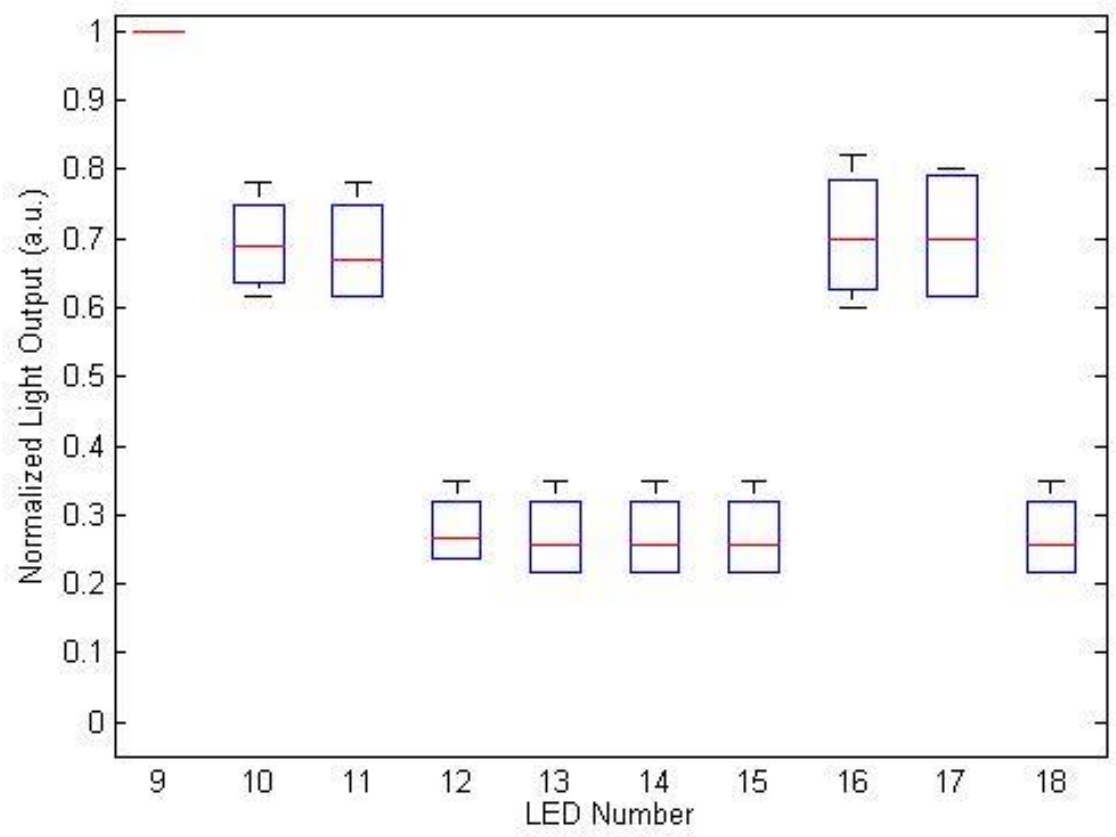

LED Chip 2 Normalized Light Output 


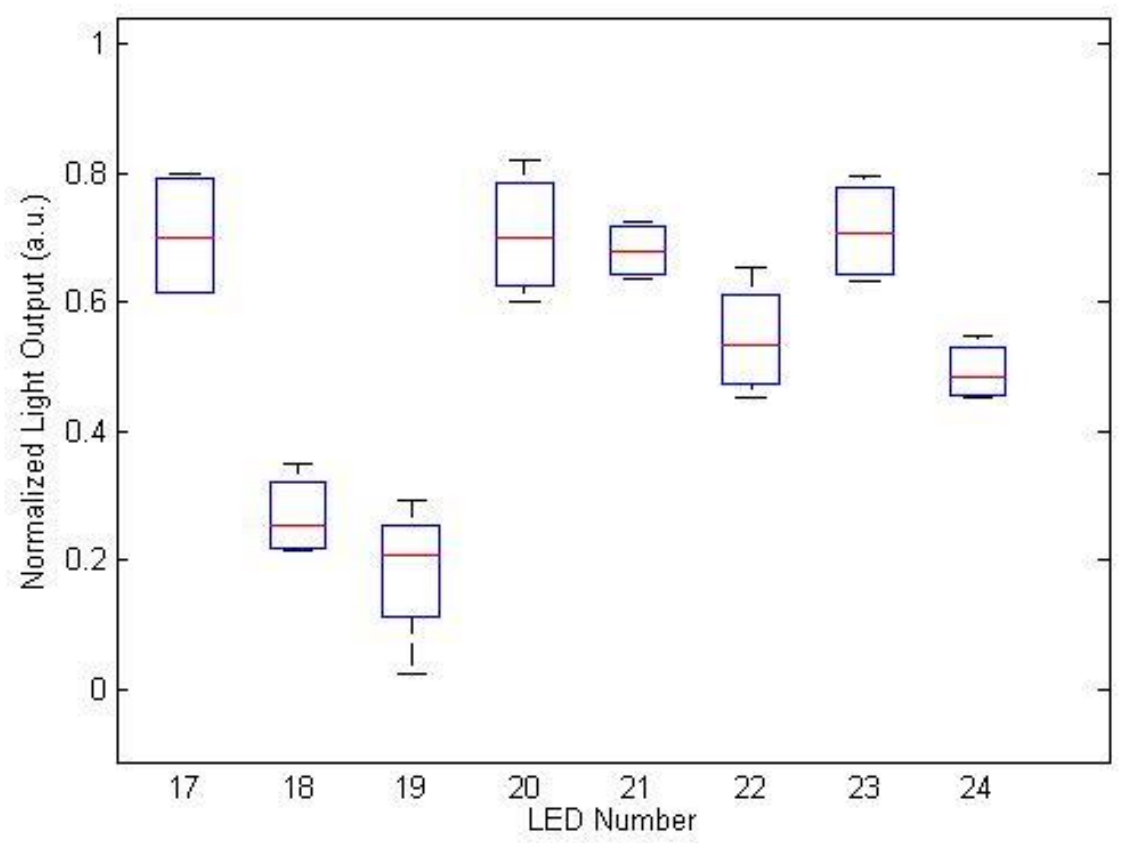

LED Chip 3 Normalized Light Output

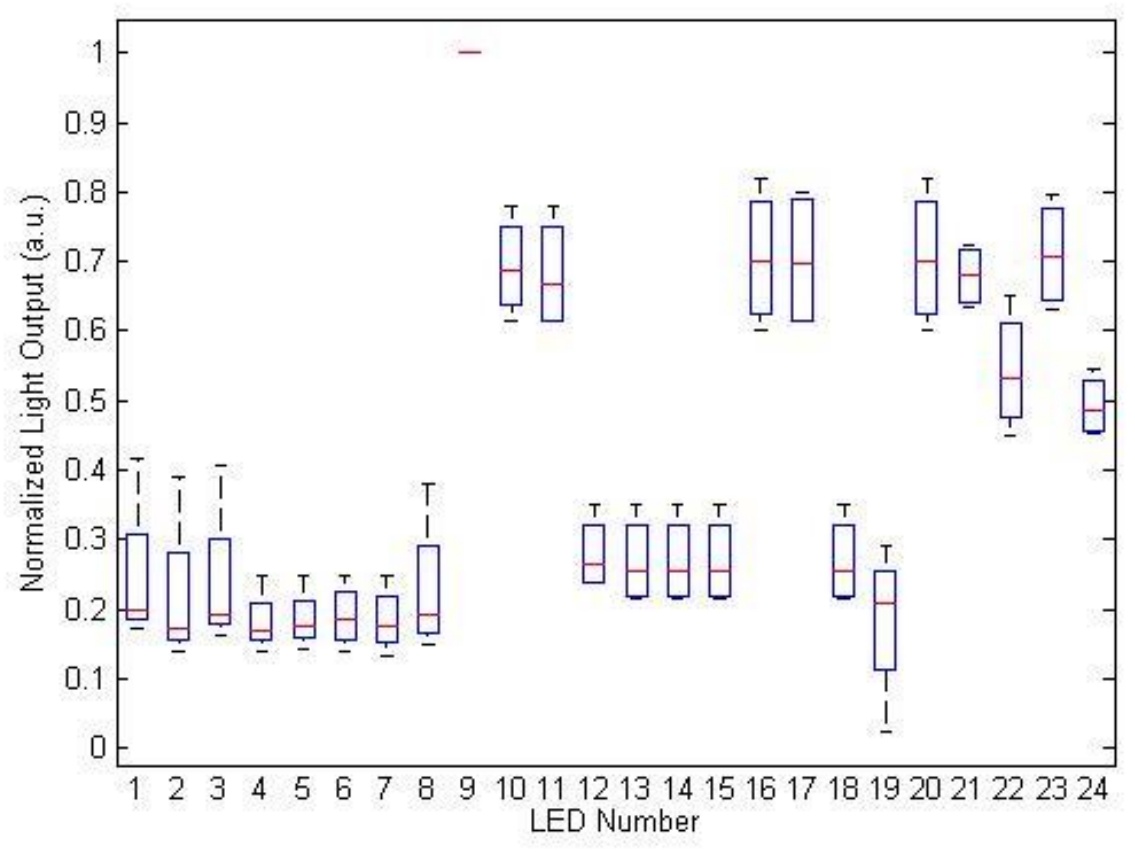

All LEDs Normalized Light Output 


\section{A-4. Individual EL Characteristics}

List of individual CIE 1931 colorimeter characteristics of each LED. Values and graphs used to verify LOCC operation. Resulting values have been highlighted to make viewing easier.

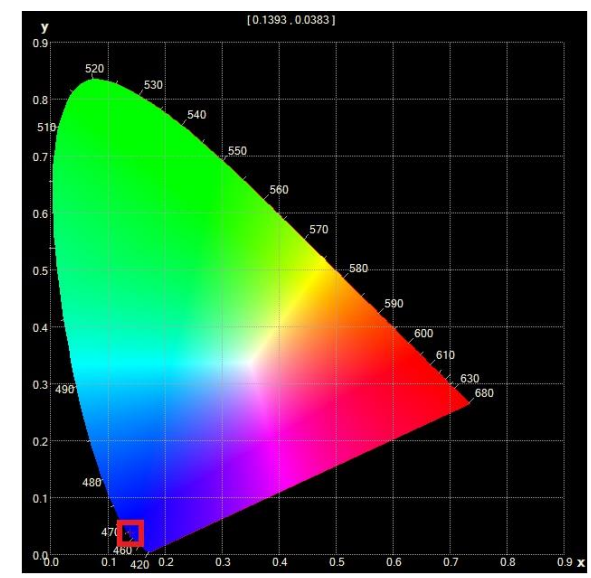

LED 1

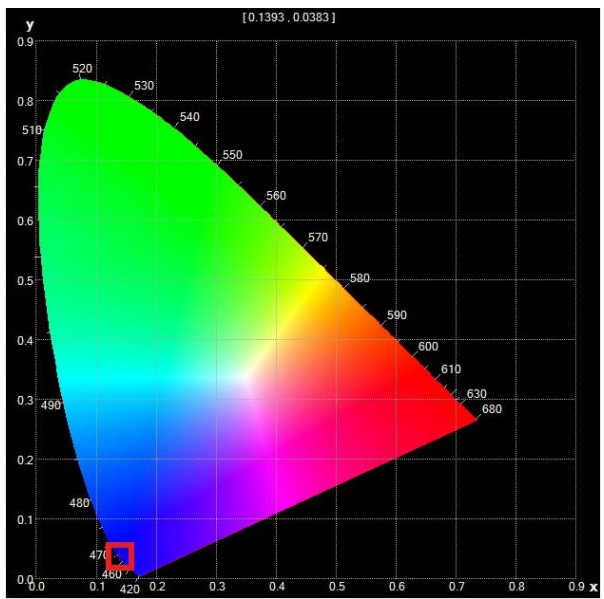

LED 3

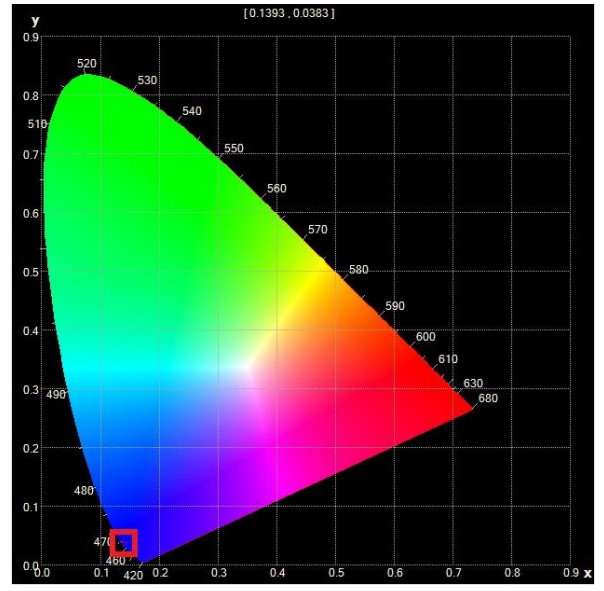

LED 5

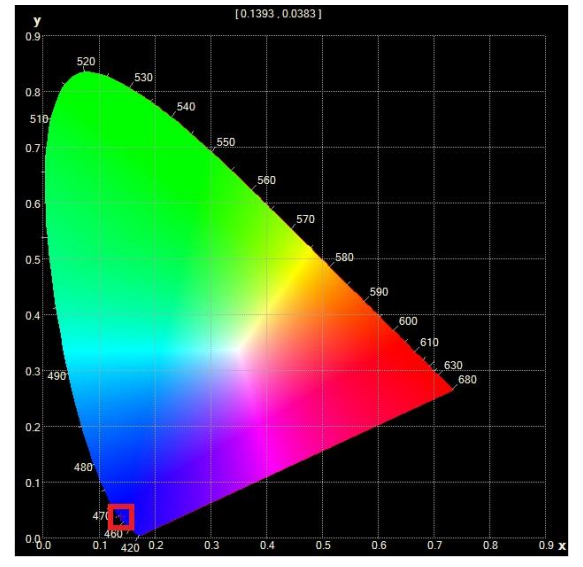

LED 2

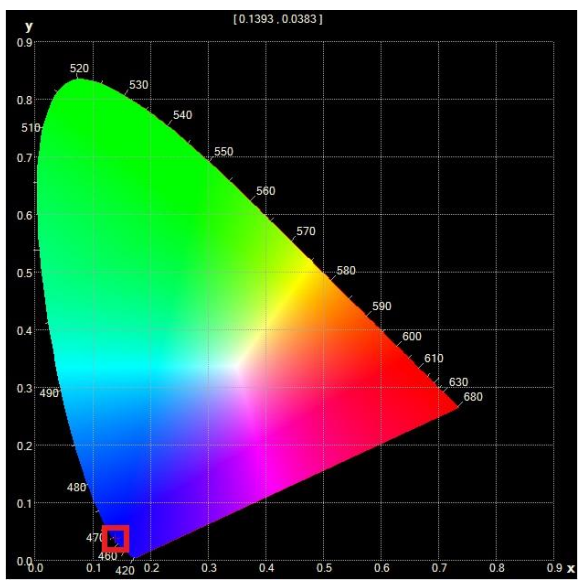

LED 4

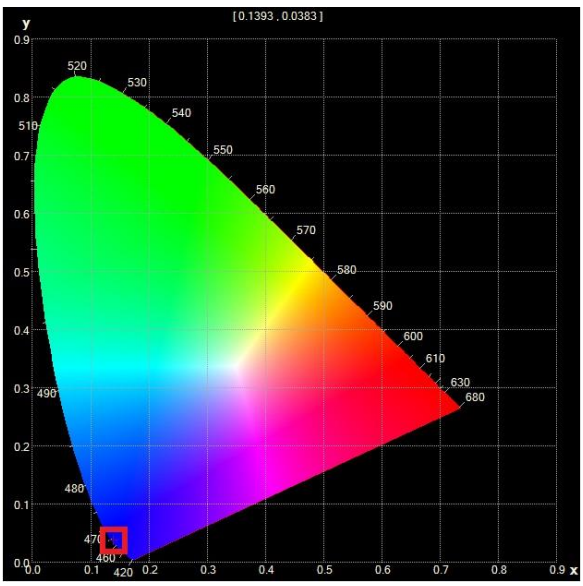

LED 6

LED 1 - 6: Commercial Evaluation of EL 


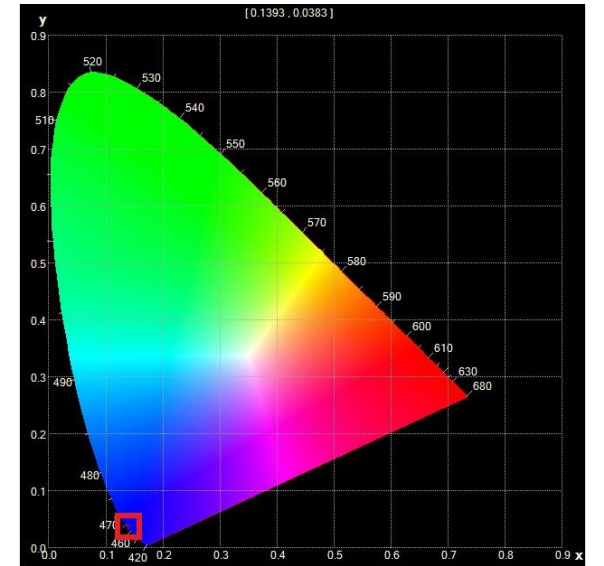

LED 9

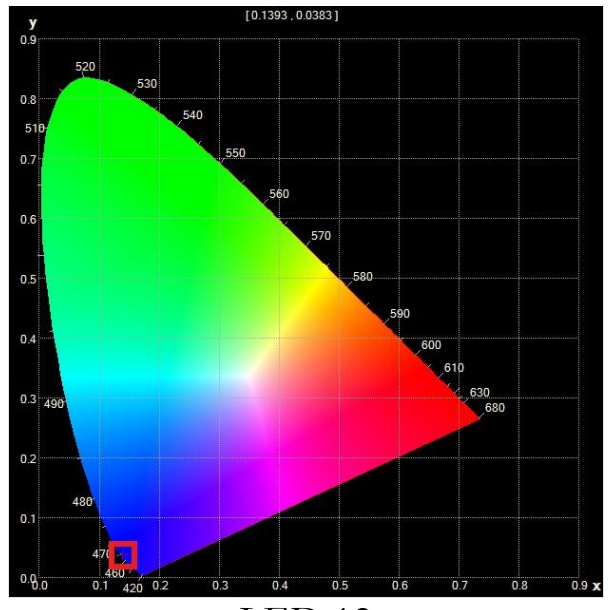

LED 12

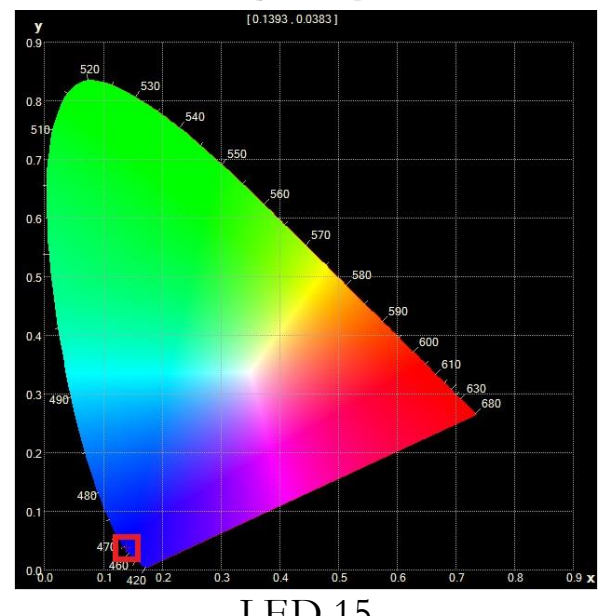

LED 9-16: Commercial Evaluation of EL

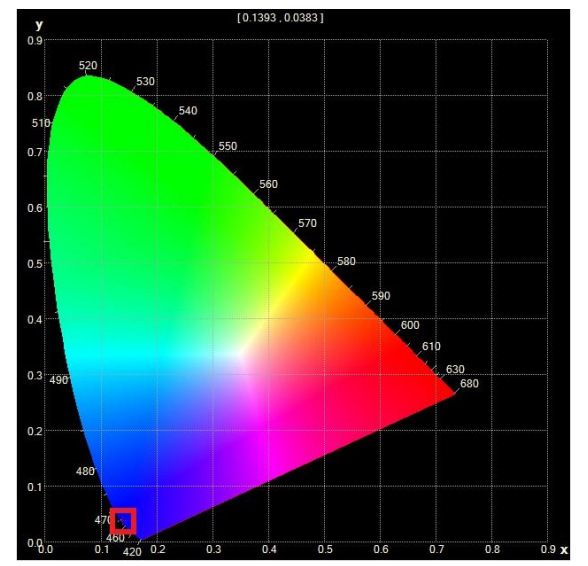

LED 10

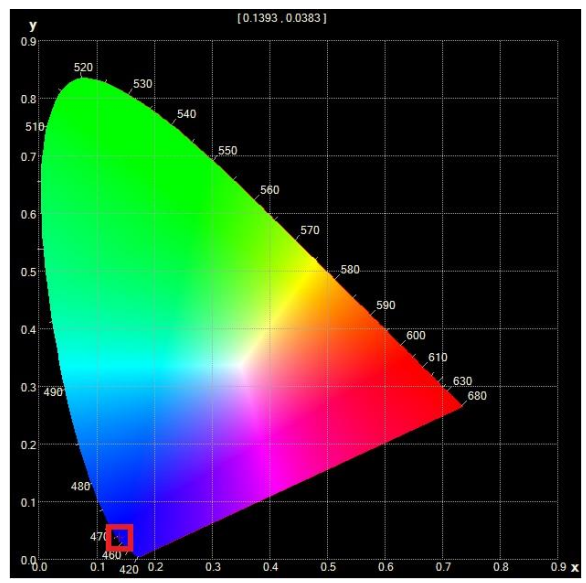

LED 14

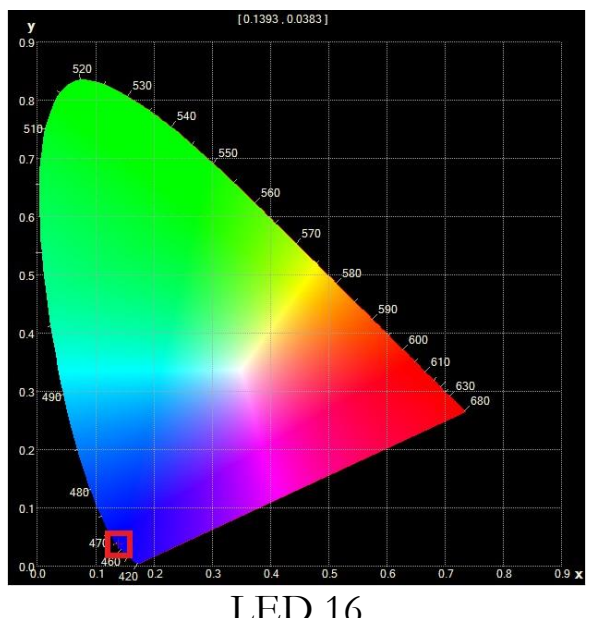

LED 16 

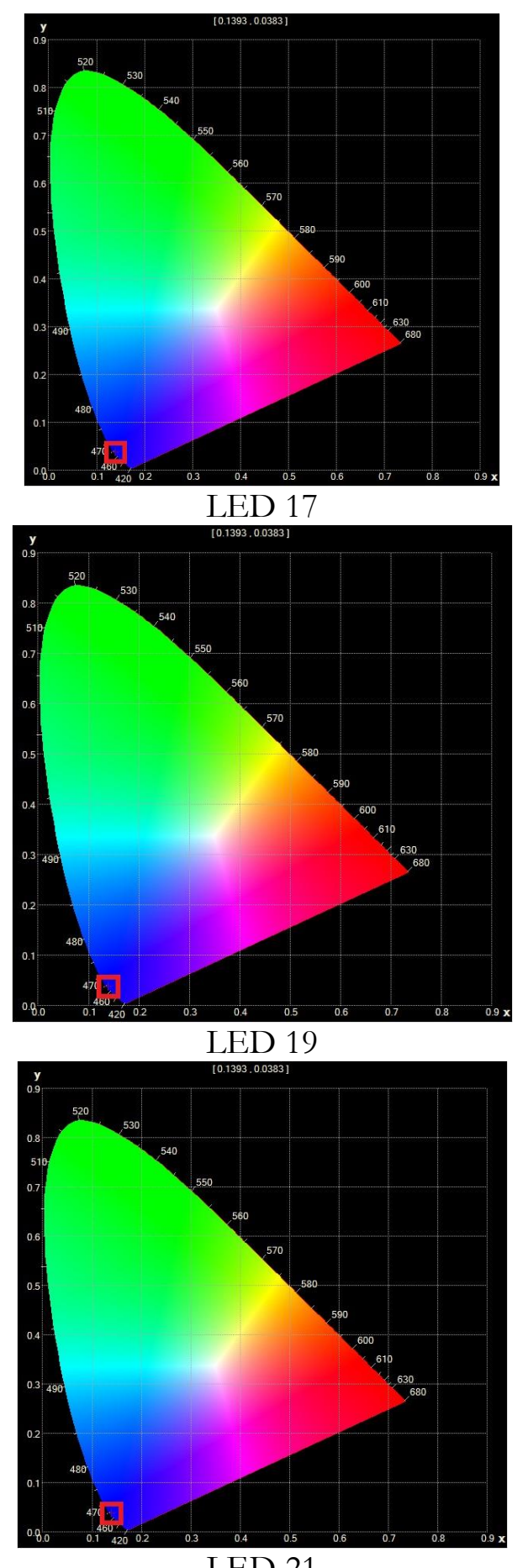

LED 21

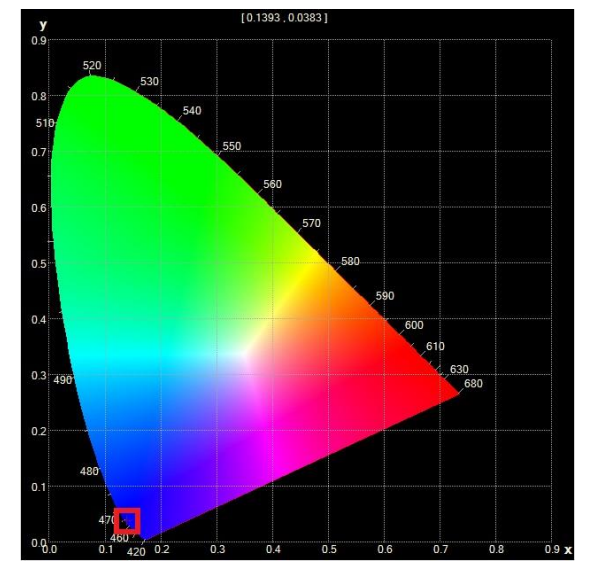

LED 18
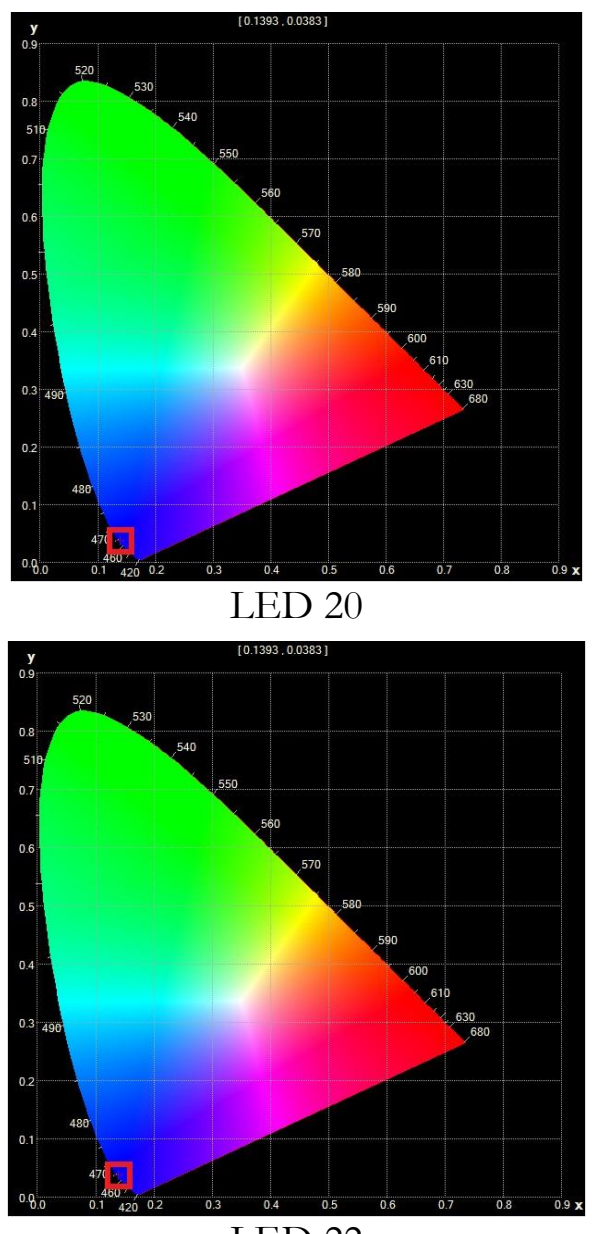

LED 22

LED 17 - 22: Commercial Evaluation of EL 

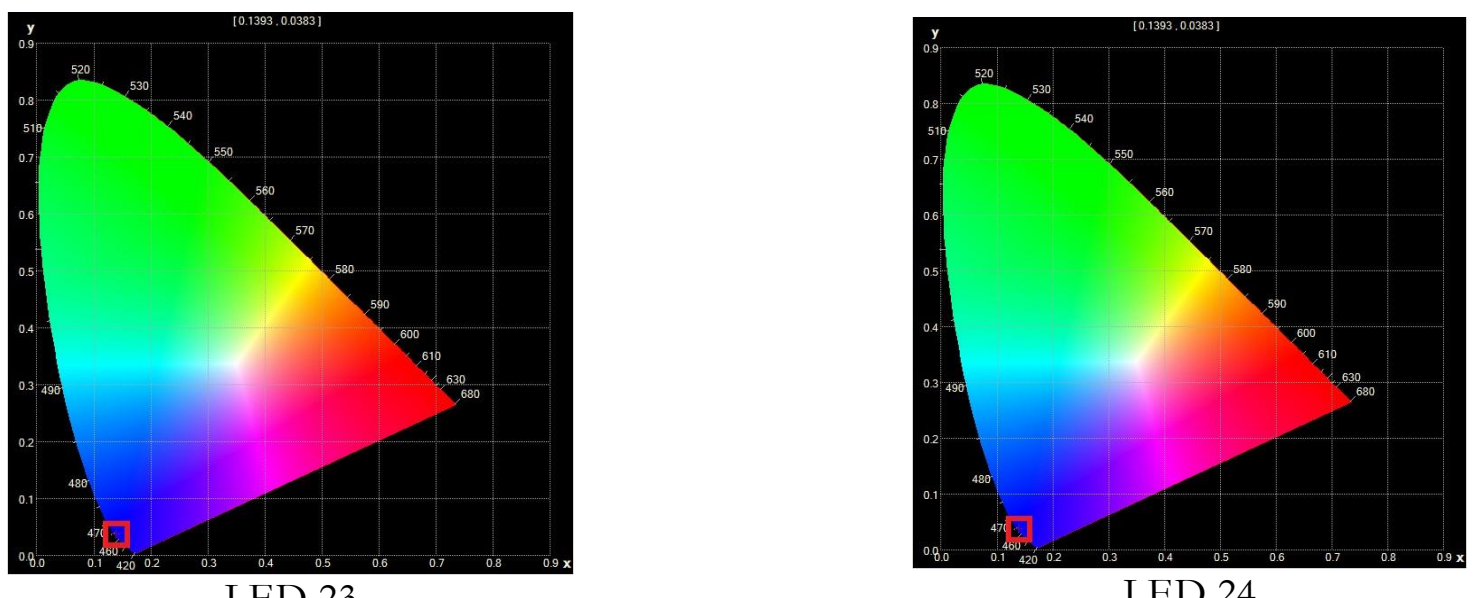

LED 24

LED 23 - 24: 4 trials and Mean and Standard Deviation 


\section{B-1. Costs}

Below a table of costs are provided to produce LOCC and the LEDs. For a detailed cost breakdown of each component please see appendix.

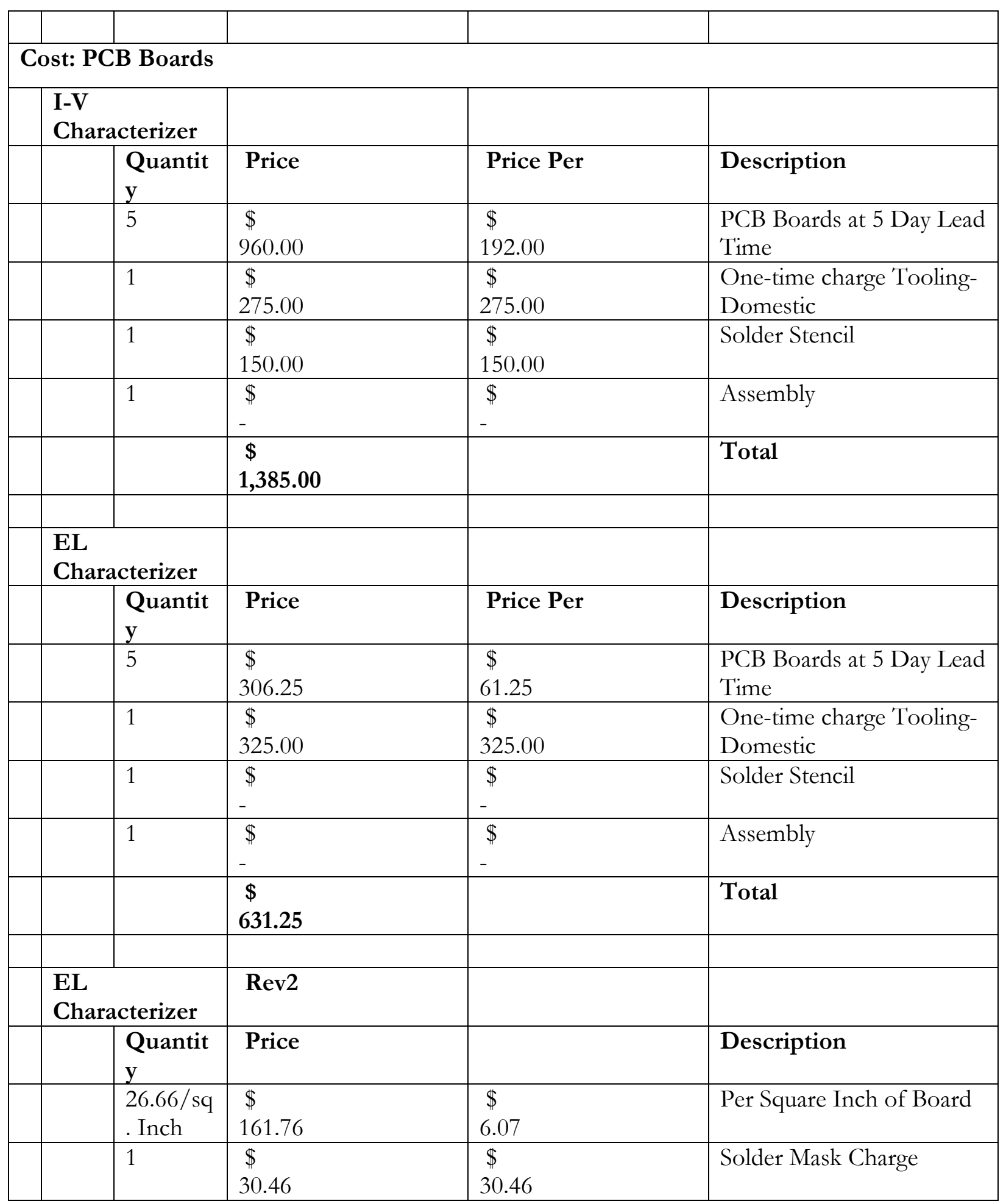




\begin{tabular}{|c|c|c|c|c|}
\hline & 2 & $\begin{array}{l}\$ \\
15.23\end{array}$ & $\begin{array}{l}\$ \\
30.46\end{array}$ & Silk Screening \\
\hline & 1 & $\begin{array}{l}\$ \\
10.51\end{array}$ & $\begin{array}{l}\$ \\
10.51\end{array}$ & Routing Charge \\
\hline & $\begin{array}{l}26.66 / \mathrm{sq} \\
\text {. Inch }\end{array}$ & $\begin{array}{l}\$ \\
0.68\end{array}$ & $\begin{array}{l}\$ \\
18.21\end{array}$ & E-Test, Double sided \\
\hline & 1 & $\$$ & $\$$ & Stencil \\
\hline & & $\begin{array}{l}\$ \\
218.64\end{array}$ & & Total \\
\hline & \multicolumn{4}{|c|}{ Components } \\
\hline & $\begin{array}{l}\text { Quantit } \\
\text { y }\end{array}$ & Price & Price Per & Description \\
\hline & $\begin{array}{l}5 \\
\text { Batches }\end{array}$ & $\begin{array}{l}\$ \\
1,037.33\end{array}$ & $\begin{array}{l}\$ \\
207.47\end{array}$ & $\begin{array}{l}\text { Components Need to } \\
\text { Produce both boards }\end{array}$ \\
\hline & & $\begin{array}{l}\$ \\
1,037.33\end{array}$ & & Total \\
\hline \multicolumn{5}{|c|}{$\begin{array}{l}\text { Cost: LED } \\
\text { Fabrication }\end{array}$} \\
\hline & \multicolumn{4}{|c|}{$\begin{array}{l}\text { Cleanroom } \\
\text { Access }\end{array}$} \\
\hline & $\begin{array}{l}\text { Quantit } \\
\text { y }\end{array}$ & Price & Price per & Description \\
\hline & 5 day & $\begin{array}{l}\$ \\
550.00 \\
\end{array}$ & $\begin{array}{l}\$ \\
110.00 \\
\end{array}$ & Cleanroom User Fee \\
\hline & & $\begin{array}{l}\$ \\
550.00\end{array}$ & & Total \\
\hline & \multicolumn{4}{|c|}{$\begin{array}{l}\text { LE } \\
\text { D }\end{array}$} \\
\hline & $\begin{array}{l}\text { Quantit } \\
\text { y }\end{array}$ & Price & Price per & Description \\
\hline & 5 & $\begin{array}{l}\$ \\
1,200.00\end{array}$ & $\begin{array}{l}\$ \\
240.00\end{array}$ & LED Substrate \\
\hline & 1 & $\begin{array}{l}\$ \\
42.50\end{array}$ & $\begin{array}{l}\$ \\
42.50 \\
\end{array}$ & $\begin{array}{l}\text { Epotek Epoxy 353-ND } 8 \\
\text { oz }\end{array}$ \\
\hline & 1 & $\begin{array}{l}\$ \\
48.00\end{array}$ & $\begin{array}{l}\$ \$ \\
48.00\end{array}$ & $\begin{array}{l}\text { Epotek Epoxy } 301-2 F L ~ \\
\text { oz }\end{array}$ \\
\hline & 20 & $\begin{array}{l}\$ \\
321.40\end{array}$ & $\begin{array}{l}\$ \$ \\
16.07\end{array}$ & $\begin{array}{l}\text { 8428-11B1-RK-TP Chip } \\
\text { carrier socket }\end{array}$ \\
\hline & 20 & $\begin{array}{l}\$ \\
660.00\end{array}$ & $\begin{array}{l}\$ \\
33.00\end{array}$ & PB-F87049 Chip Carrier \\
\hline & 20 & $\begin{array}{l}\$ \\
321.40\end{array}$ & $\begin{array}{l}\$ \\
16.07\end{array}$ & 8428-21B1-RK-TR \\
\hline & & $\begin{array}{l}\$ \\
2,593.30\end{array}$ & & Total \\
\hline
\end{tabular}




\begin{tabular}{|c|c|c|}
\hline $\begin{array}{l}\text { Cost: } \\
\text { Total } \\
\end{array}$ & & \\
\hline & $\begin{array}{l}\$ \\
6,415.52\end{array}$ & Total \\
\hline
\end{tabular}

\section{B-2.}

LOCC Raspberry Pi commander and binary converter.

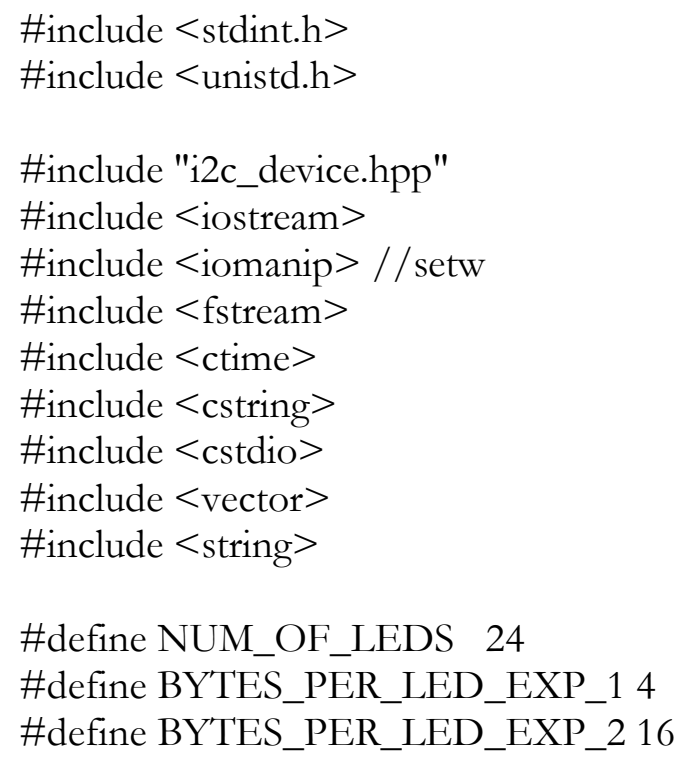

//pass filename to convert binary to readable data 


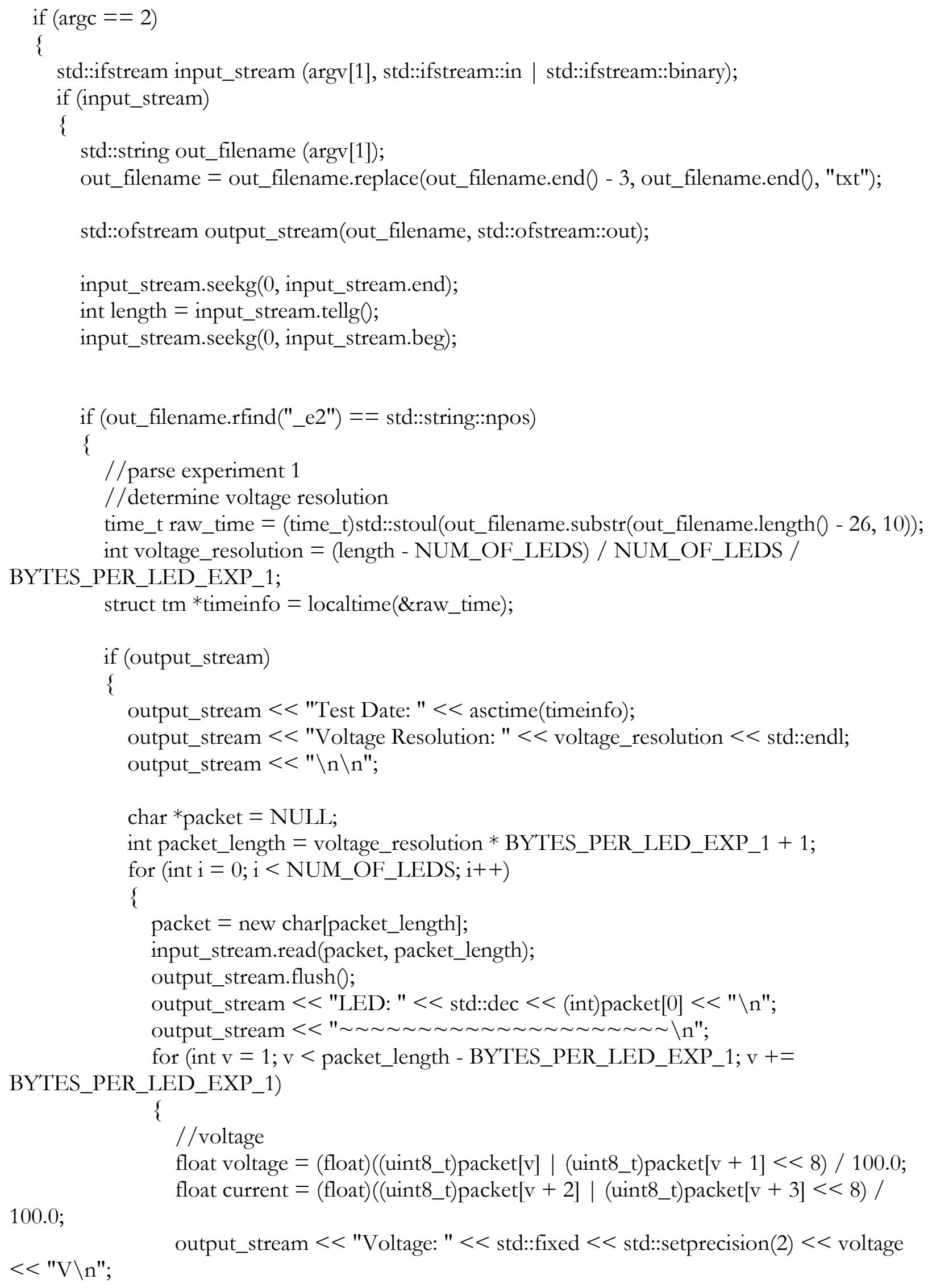




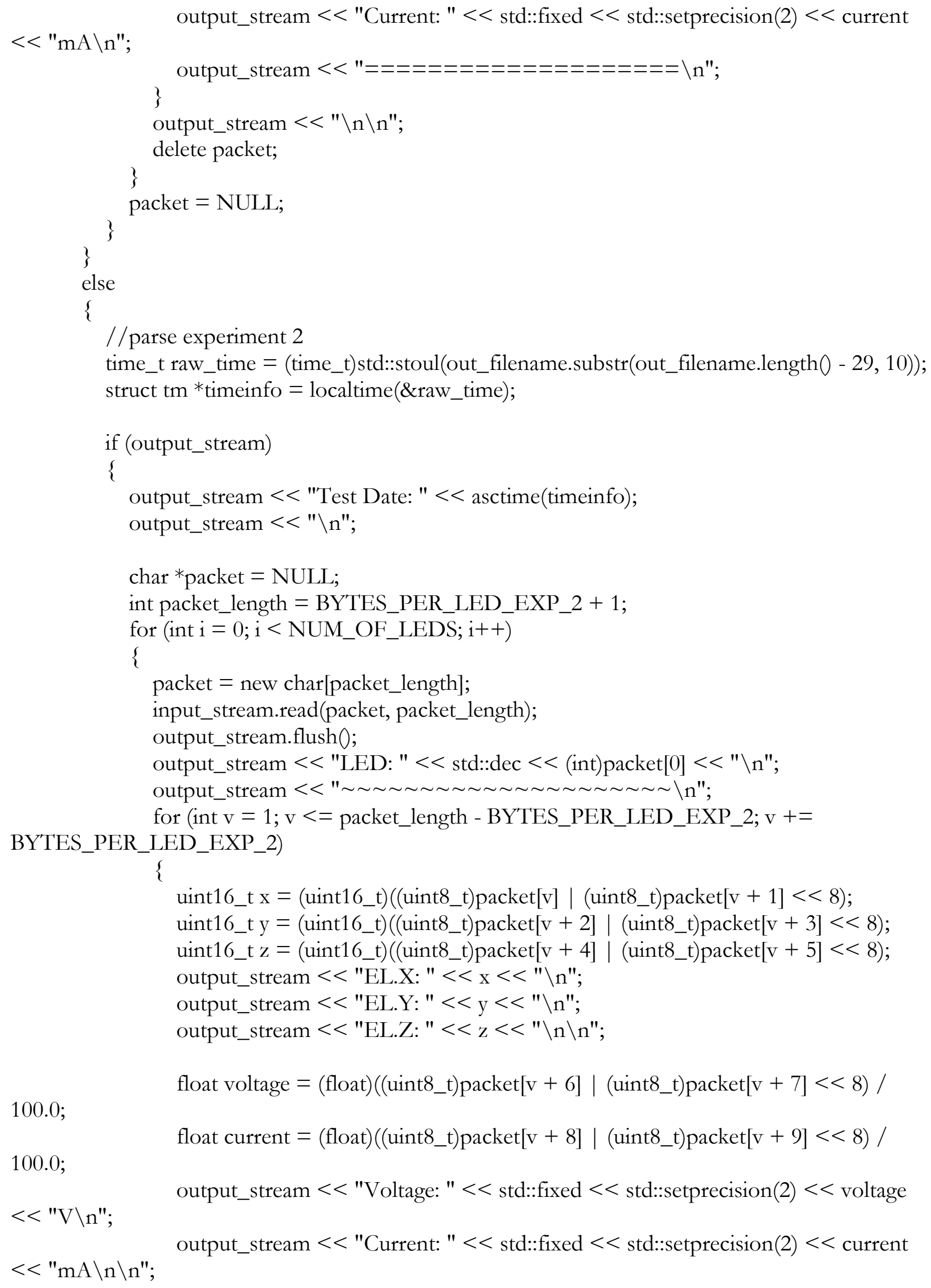




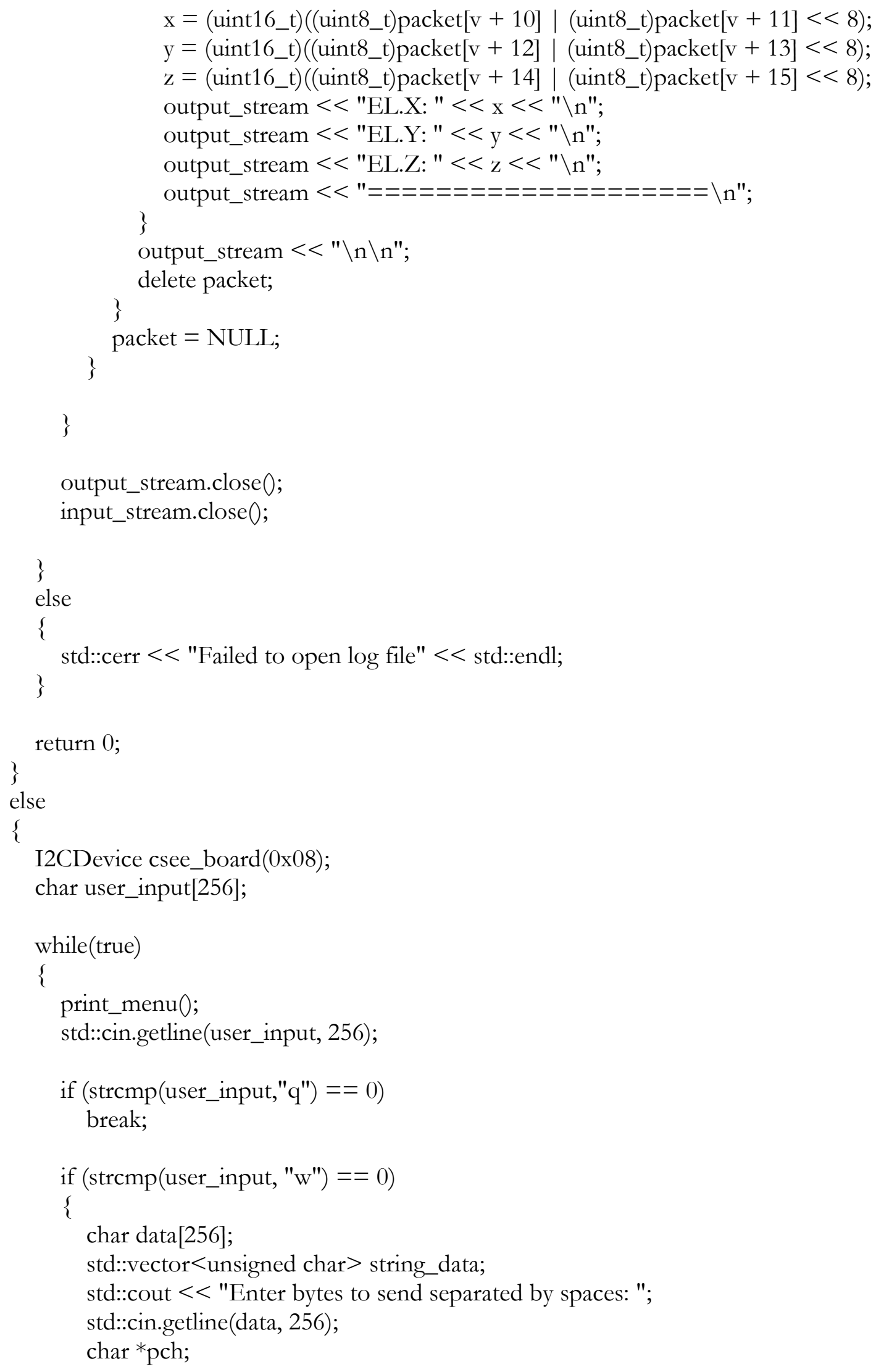




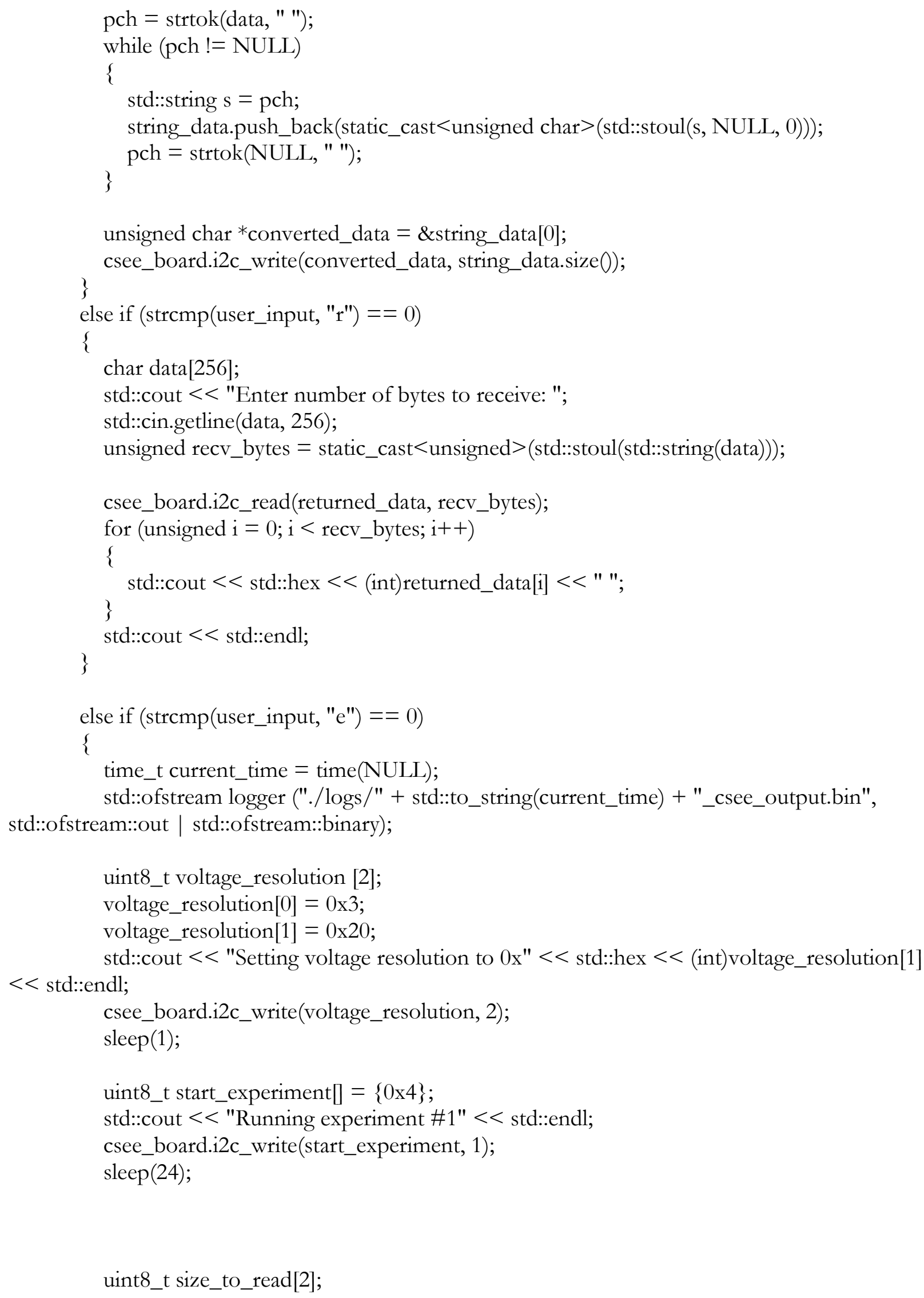


uint8_t get_size_to_read $]=\{0 \mathrm{x} 1\}$;

csee_board.i2c_write(get_size_to_read, 1);

sleep(1);

csee_board.i2c_read(size_to_read, 2);

uint16_t size $=($ size_to_read[0] $<<8) \mid$ size_to_read[1];

std::endl;

std::cout $<<$ "CSEE firmware reports " $<<$ std::dec $<<$ size $<<$ " bytes available" $<<$

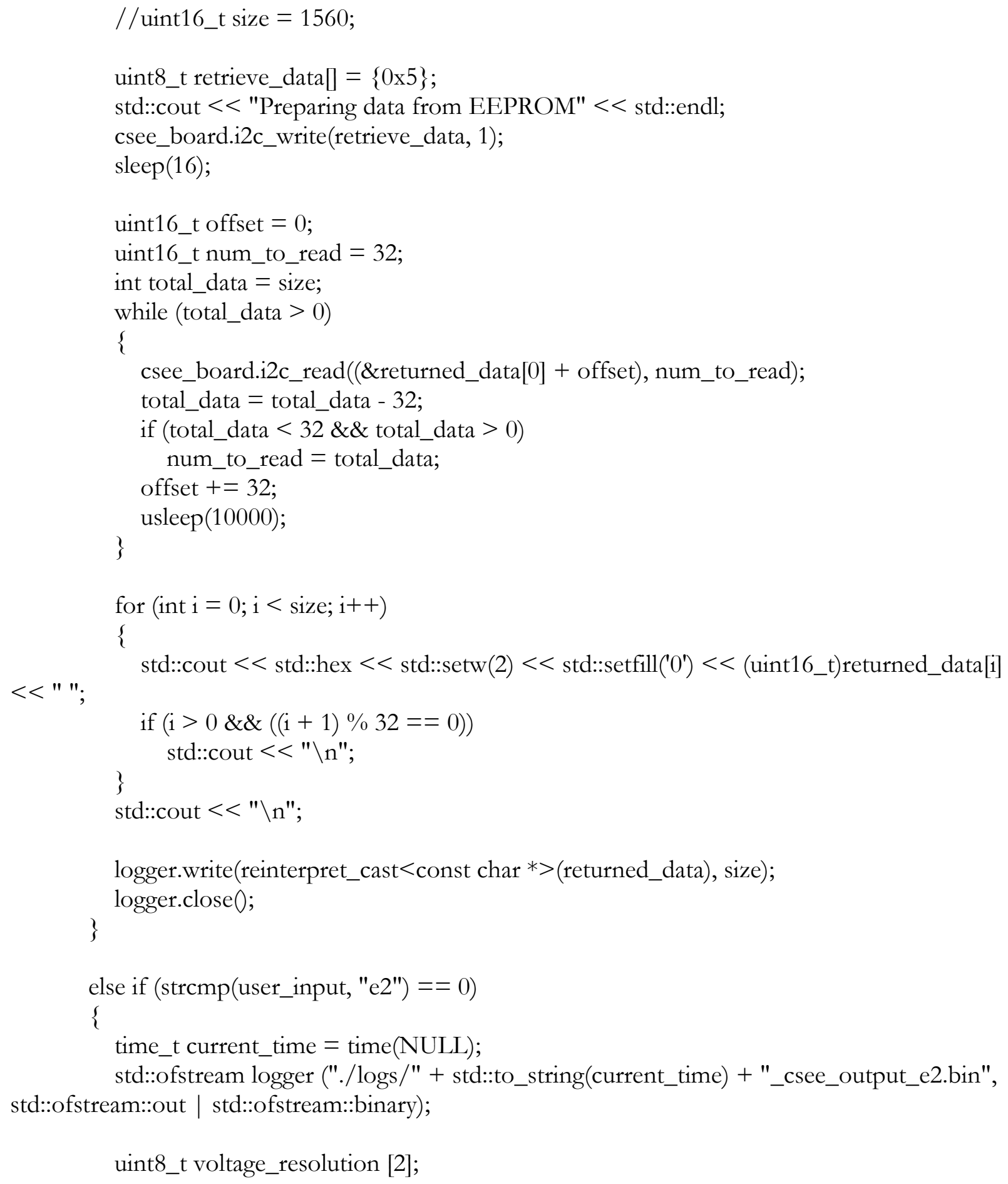


voltage_resolution $[0]=0 \times 3$;

voltage_resolution[1] $=0 \times 20$;

std::cout $<<$ "Setting voltage resolution to $0 \mathrm{x} "<<$ std::hex $<<$ (int)voltage_resolution[1] $<<$ std::endl;

csee_board.i2c_write(voltage_resolution, 2);

sleep(1);

uint8_t start_experiment $[=\{0 \times 6\}$;

std::cout $<<$ "Running experiment \#2" $<<$ std::endl;

csee_board.i2c_write(start_experiment, 1);

$\operatorname{sleep}(120)$;

std::endl;

uint8_t size_to_read[2];

uint8_t get_size_to_read $]=\{0 \mathrm{x} 1\}$;

csee_board.i2c_write(get_size_to_read, 1);

sleep(1);

csee_board.i2c_read(size_to_read, 2);

uint16_t size $=($ size_to_read[0] $<<8) \mid$ size_to_read[1];

std::cout $<<$ "CSEE firmware reports " $<<$ std::dec $<<$ size $<<$ " bytes available" $<<$

/ / uint16_t size $=1560 ;$

uint8_t retrieve_data $]=\{0 \times 5\}$;

std::cout $<<$ "Preparing data from EEPROM" < < std::endl;

csee_board.i2c_write(retrieve_data, 1);

sleep(16);

uint16_t offset $=0$;

uint16_t num_to_read $=32$;

int total_data = size;

while (total_data $>0$ )

\{

csee_board.i2c_read((\&returned_data[0] + offset $)$, num_to_read);

total_data $=$ total_data -32 ;

if (total_data $<32 \& \&$ total_data $>0$ )

num_to_read $=$ total_data;

offset $+=32$;

\}

usleep(10000);

for (int $\mathrm{i}=0 ; \mathrm{i}<$ size $; \mathrm{i}++$ )

\{

$<<"$ ";

std::cout $<<$ std::hex $<<$ std::setw $(2)<<$ std::setfill('(0') $<<$ (uint16_t)returned_data[i]

if $(i>0 \& \&((i+1) \% 32==0))$ 


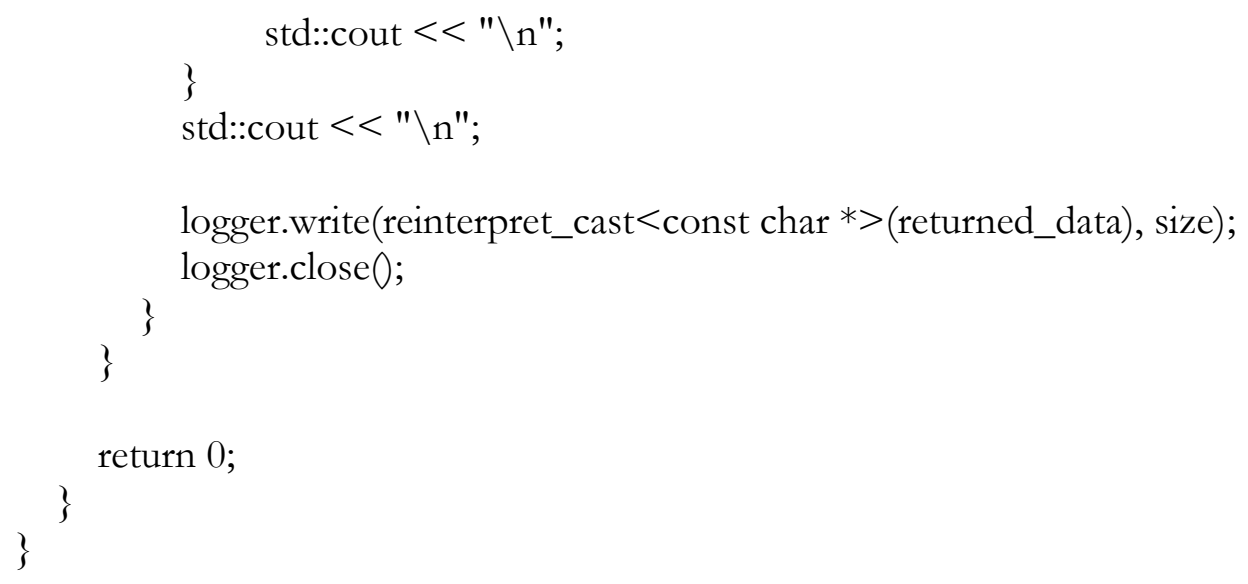

B-3.

LOCC EL Evaluation algorithm.

clear all

clc

img $=$ imread('CIExy1931.bmp');

EL_1 = xlsread('LOCC_EL_1.xlsx');

EL_2 = xlsread('LOCC_EL_2.xlsx');

EL_3 = xlsread('LOCC_EL_3.xlsx');

EL_4 = xlsread('LOCC_EL_4.xlsx');

XYZ455 = [15327, 4000, 88192];

$X Y Z 465=[487,64,2599]$;

$\mathrm{XYZ510}=[250,26,1196]$;

$\mathrm{T}(:, 1)=\mathrm{XYZ455}$

$\mathrm{T}(:, 2)=\mathrm{XYZ465}$

$\mathrm{T}(:, 3)=\mathrm{XYZ510}$

$\mathrm{ADC1}=[65535,65535,65535] ;$

ADC2 = [46519, 65535, 65535];

$\mathrm{ADC} 3=[16812,65535,34332]$; 


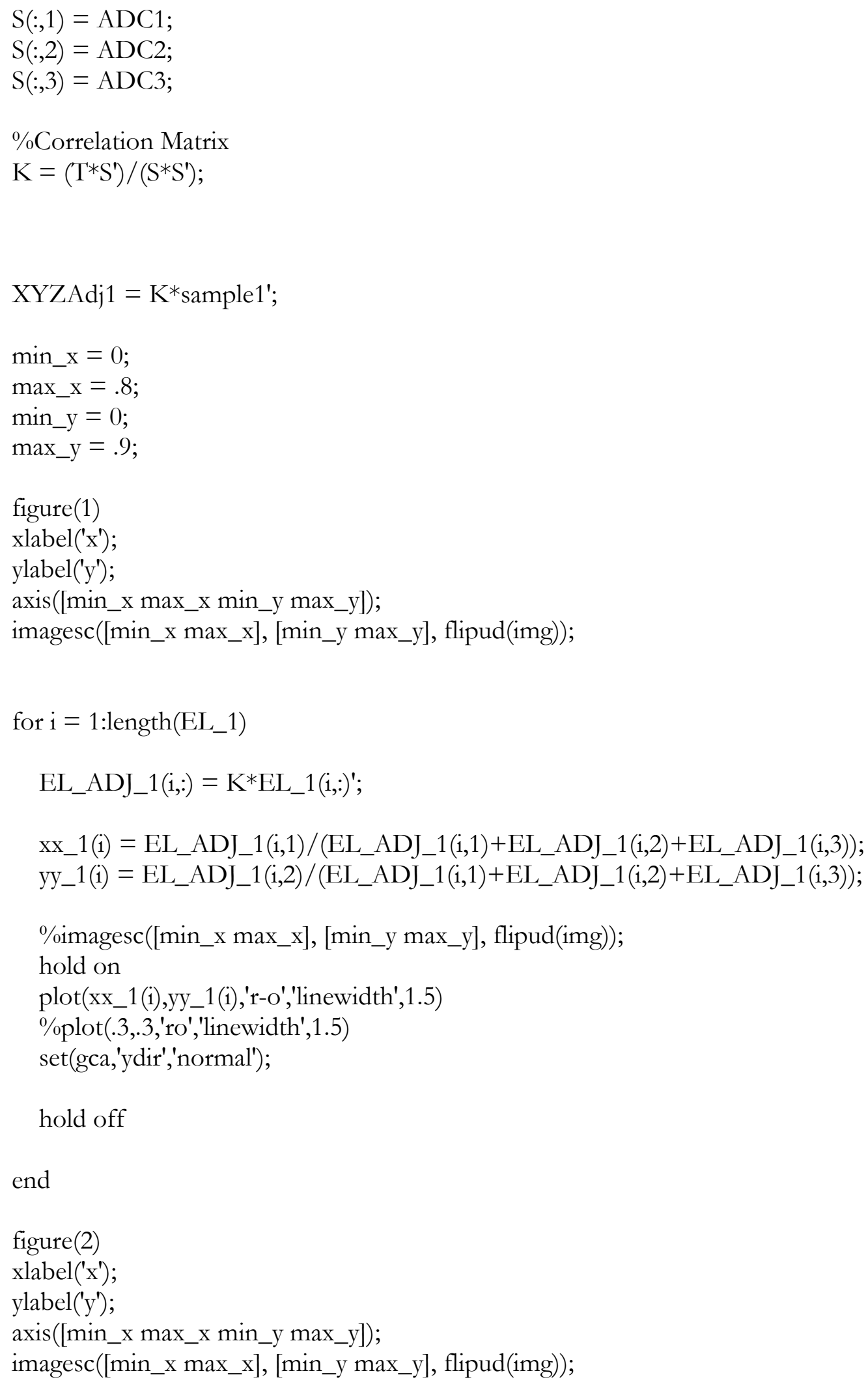




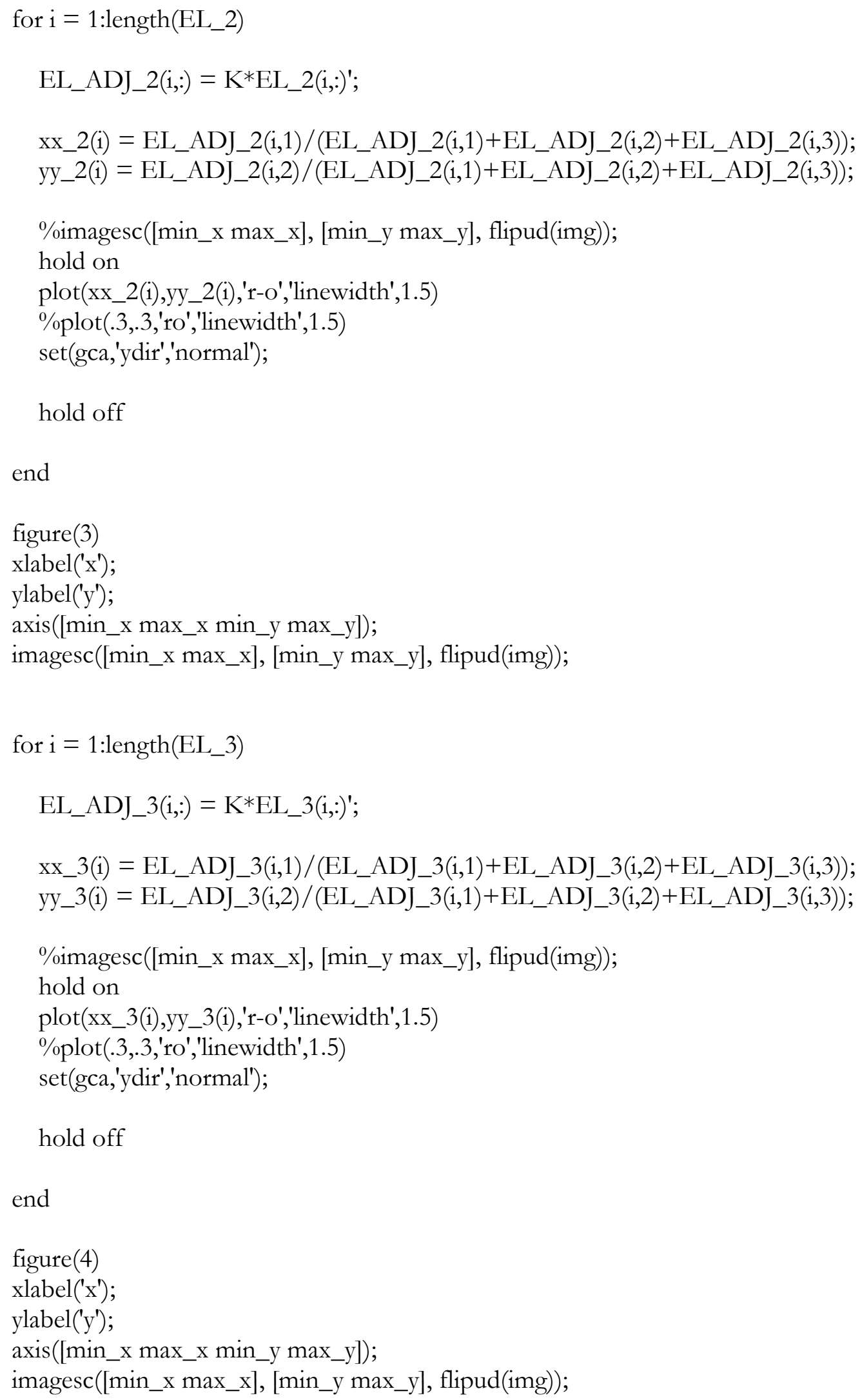




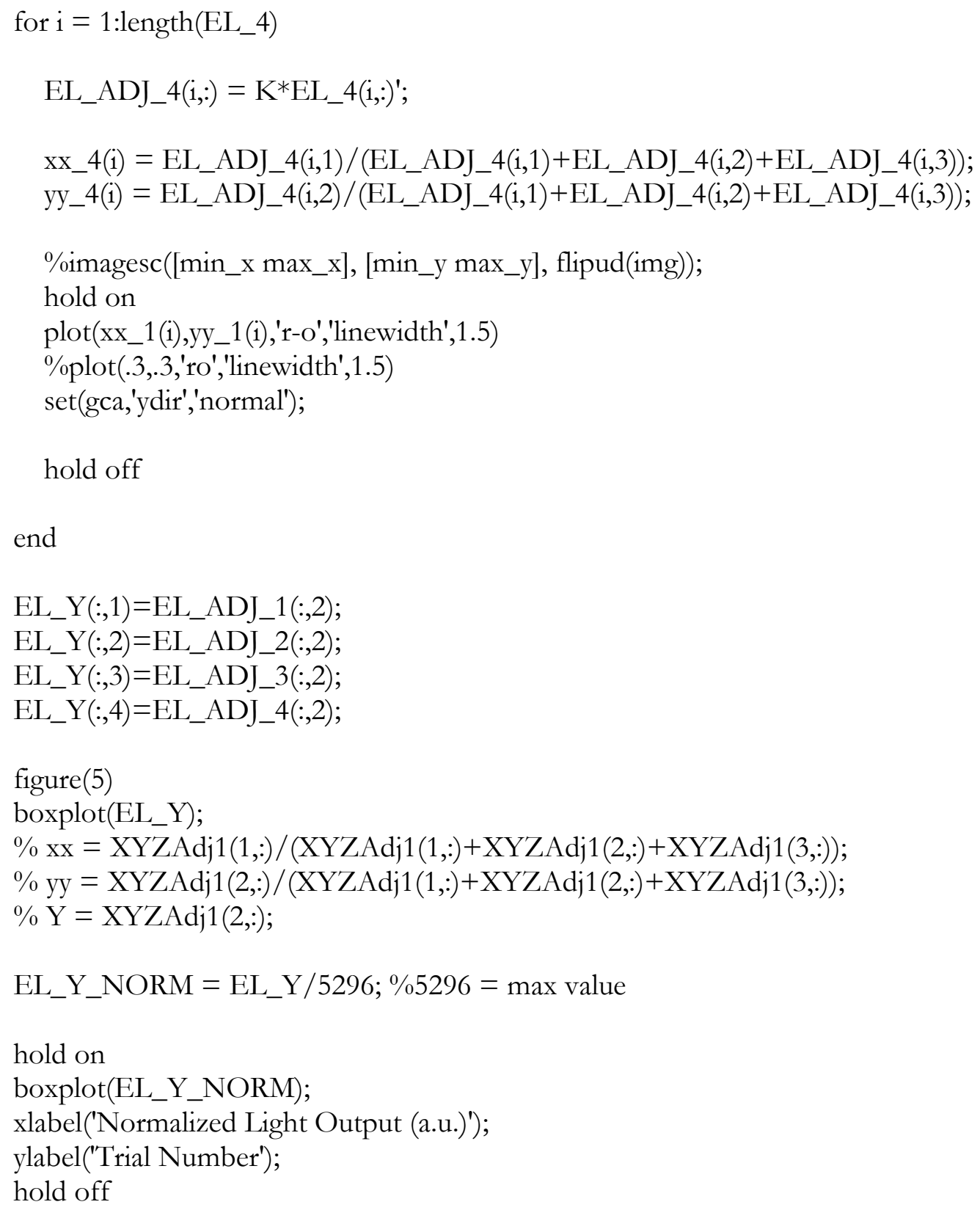

